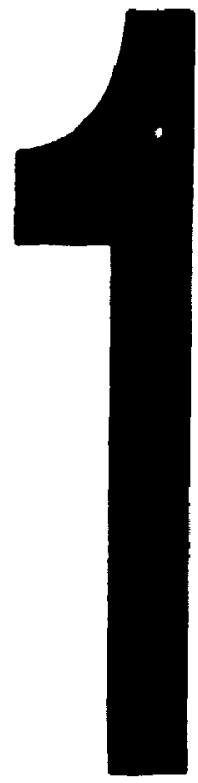

PM-1 3K" " 4 " PHOTOERAPHIC MICROCOPY TARGET MaS 10100 AMSI/1SO "2 EOUIVALENT

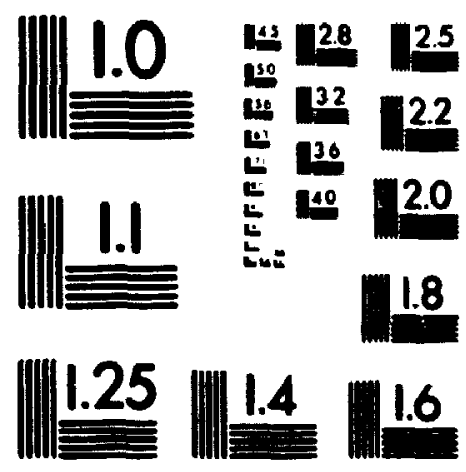

PAECISIONEM RESOLUTION TARCETS 


\title{
MECHANICS AND DESIGN OF A VARIABLE STIFFNESS ACTUATOR FOR USE IN IMPEDANCE CONTROL
}

by

\section{CHAD ENGLISH, B.Eng.}

\author{
A thesis submitted to \\ the Faculty of Graduate Studies and Research \\ in partial fulfillment of \\ the requirements for the degree of \\ Master of Engineering (Mechanical)
}

Department of Mechanical and Aerospace Engineering

Ottawa-Carleton Institute

for Mechanical and Aerospace Engineering

\author{
Carleton Univeristy \\ Ottawa, Ontaric \\ April 15, 1996 \\ (C) copyright \\ 1996, Chad English
}


National Libray

of Cenada

Acquisitions and

Bibliographic Senices Branch

395 wewhon swect

Onawe Onterio

KiAON
Bibliotheque nationale

du Canada

Direction des acquisitions et

des senvices bibliographiques

395. ne Writingon

Orive (Ontrió)

K1AONA

rout is vomenninge

The author has granted an irrevocable non-exclusive licence allowing the National Library of Canada to reproduce, loan, distribute or sell copies of his/her thesis by any means and in any form or format, making this thesis available to interested persons.

The author retains ownership of the copyright in his/her thesis. Neither the thesis nor substantial extracts from it may be printed or otherwise reproduced without his/her permission.
L'auteur a accordé une licence irrévocable et non exclusive permettant a la Bibliotheque nationale du Canada de reproduire, prêter, distribuer ou vendre des copies de sa these de quelque manière et sous quelque forme que ce soit pour mettre des exemplaires de cette these a la disposition des personnes intéressées.

L'auteur conserve la propriété du droit d'auteur qui protège sa thèse. Ni la these ni des extraits substantiels de celle-ci ne doivent être imprimés ou autrement reproduits sans son autorisation.

ISBN $\quad 0-612-13838-0$ 
Nom Chad Enalish

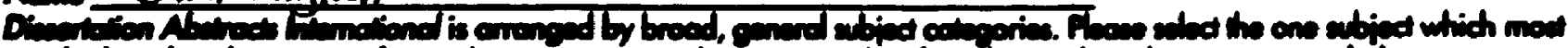

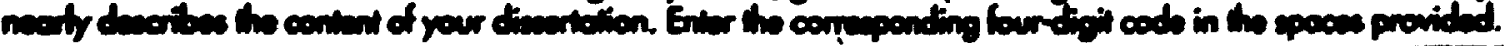
Meshanicul Engineering 051418 U.MI

Surined Composion

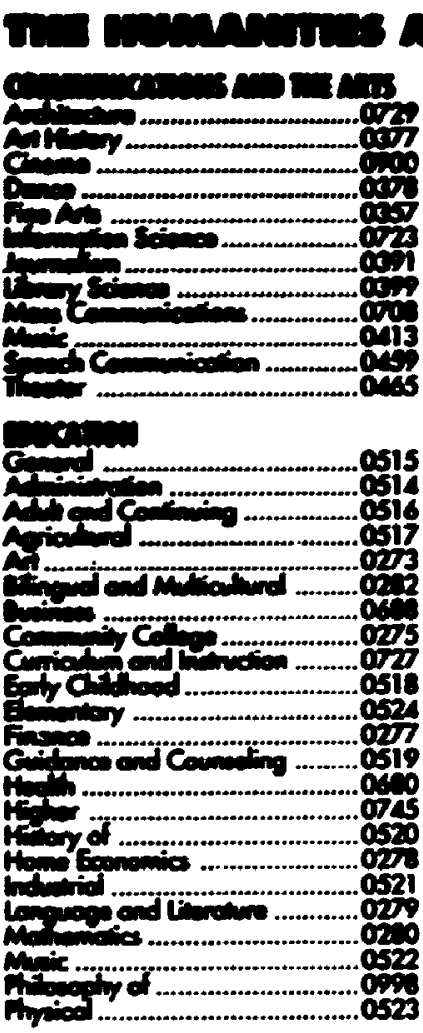

(1)
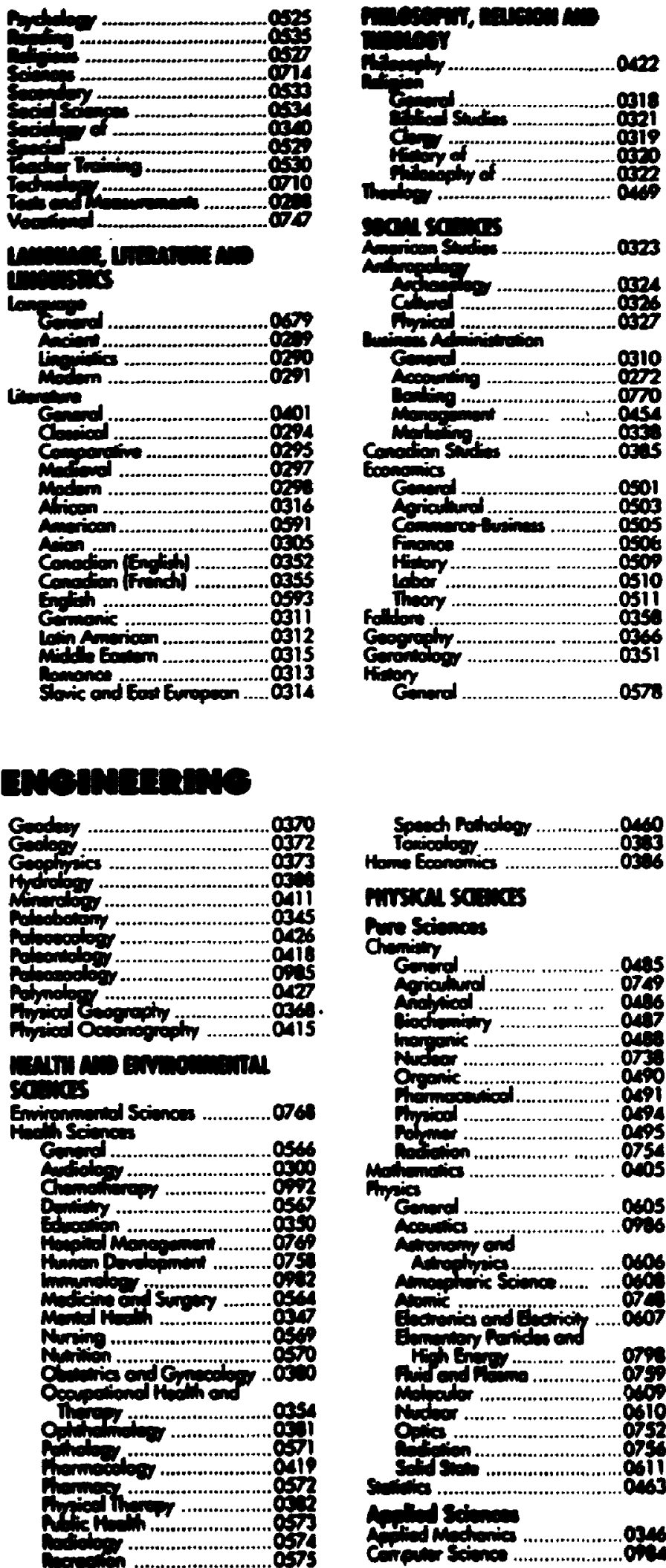

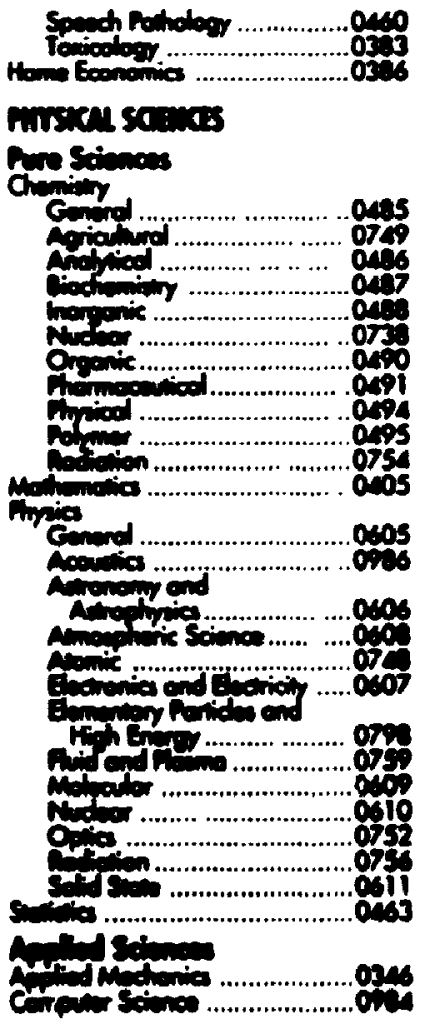

Engineving:

Sondend ...........................0537

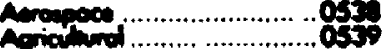

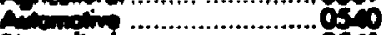

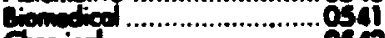

Granod ………………......oset

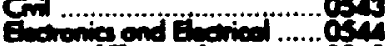

Hot end Thermod, memics ...gon

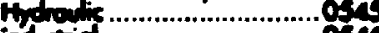

cos

Antive .............................0S

moritsoive ...............98

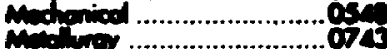

ming

Nude ...............................858

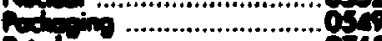

andon

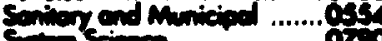

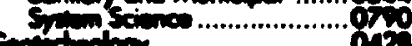

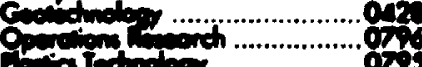

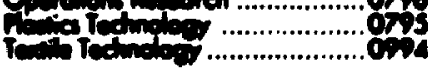

covernat

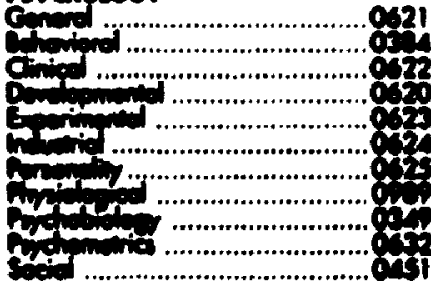


The undersigned recommend to the Faculty of Graduate Studies and Research acceptance of the thesis

"MECHANICS AND DESIGN OF A VARIABLE STIFFNESS ACTUATOR FOR USE IN IMPEDANCE CONTROL"

submitted by Chad English, B. Eng.

in partial fulfillment of the requirements for

the degree of Master of Engineering (Mechanical)
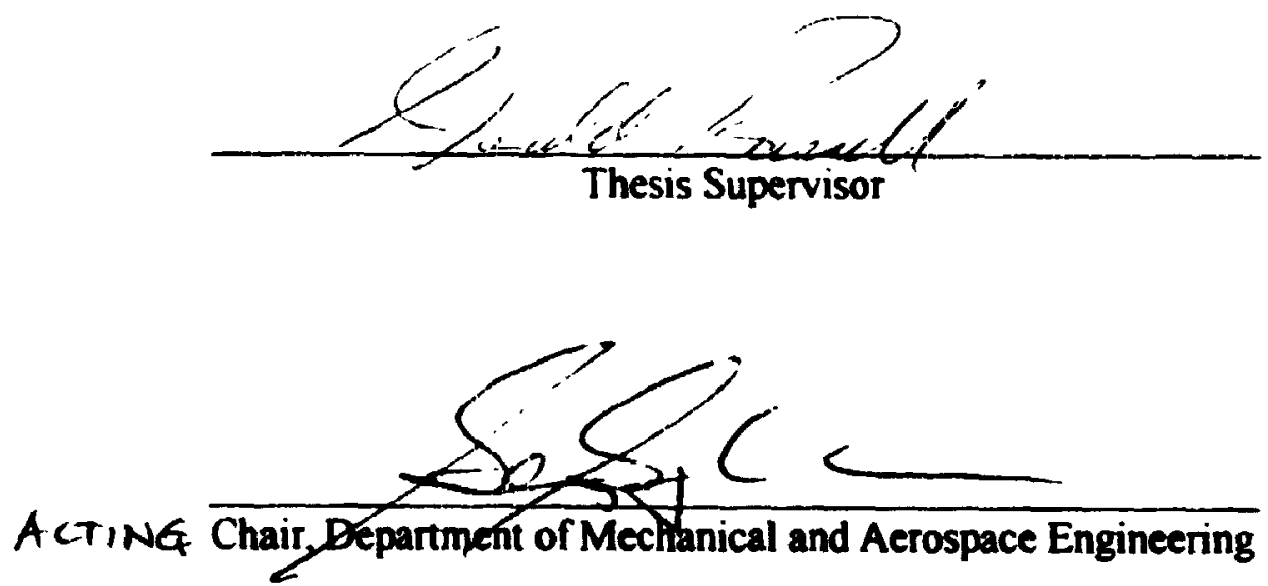

Carleton University

May 11, 1996

ii 


\section{Abstract}

Perhaps the greatest issue in the field of robotics today is the performanie of contact tasks. Impedance control allows a unified approach to both sontact and noncontact tasks and deals with control from the point of view of natural motion. The focus of this thesis is the development of a robotic joint with variable impedance, specifically the joint stiffness. Antagonistic actuation permits the use of mechanical components to modulate the stiffness. The work emphasizes the application of this design to upper limb prosthetics.

Kinematic analysis of the proposed design suggests that joint stiffness and position can be decoupled through the use of quadratic springs. Existing technology allows the creation of these springs with specific force-deflection relations. Geometric constraints limit the variable stiffness ability. Beyond these limits the joint acts as a rigid manipulator. Simulations indicate improved ability of this design over existing manipulators and prostheses. 


\section{Acknowledgments}

Originally I had no intention of pursuing a Master's degree. Like most undergrads, I was quite weary of the work and stress that are associated with a bachelor's degree in engineering. Moreover, the undergraduate program in Nova Scotia spans five years and two universities. In my case, these were Acadia University and the Technical University of Nova Scotia (TUNS), two difficult but well rated schools. By the time I finished, I felt that I had moved around enough and spent more time than I wanted in school. It was time to get a job. How wrong I was.

I consider the decision to pursue a Master's degree as the best decision I have made in my life. As I look back, I realize how ignorant I was of what it means to do graduate work. It is not simply a more intense version of an undergraduate degree. In fact, I have found it to be much less intense. Yes, there is pressure to get work done, but it is not in the form of a series of assignments and tests that judge you as though you are some product that must meet minimum specifications or be discarded. Perhaps the greatest part of graduate work is knowing that I am treading on new ground. I cannot simply look up the results in the back of a book to see if I am right. I don't feel the frustration, or paranoia, that everyone else understands the work but me. In this case, I (hopefully) understand it the best. It takes a solid understanding of the concepts to do research and create my own answers. This is certainly not a task I could perform on my own. There are many people who have heiped me along the way.

First, and foremost, I'd like to thank my supervisor, Dr. Donald Russell. It is commonly said that one's satisfaction with thesis work lies heavily on the choice of supervisors. I am positive that everyone would love their work if they had a supervisor like Dr. Russell. I enjoy it so much that he has persuaded me to continue into a Ph.D. with him. He has an uncanny ability to know what level of understanding someone has and explain things at that level. I always leave his office satisfied with the answers I get or have a clear direction for my work. Most importantly, he seems very interested and excited about the work. A quick five minute question often turns into a two hour discussion, usually leading far from the original topic. Perhaps this connection stems from our similar background at TUNS. Perhaps it is just in his nature. In either case, my respect and admiration go out to $\mathrm{Dr}$. Russell, both as a supervisor and as a person. Thank you for making my time at Carleton a good one.

I also must thank Dr. Marek Kujath at TUNS for convincing me to pursue my Master's degree. He was quite helpful in suggesting jobs in robotics and aerospace, but also expressed his confidence that I could go on to graduate work. It was Dr. Kujath who originally got me interested in robotics, space mechanics, and dynamic systems in general. I certainly would not be here if it weren't for his classes or his confidence in my abilities. 
I thank my wife Kathy for putting up with me during this stressful time. We were married in the middle of my degree and never had time to enjoy our new life together as do most newlyweds. Between the two of us, we haven't had much free time to spend together. It was my devotion to her that gave me the drive to finish my work. There may be some competition there too. She is more successful now than I can hope to be at anytime in the near future. I certainly could not have handled her workload, both school and business, when I was her age. Nor could I have been so successful at it. Her motivation and drive have been an inspiration to me and 1 am continually amazed at the accomplishments she achieves. I hope she knows how proud I am of her and how much I love her.

Finally, I thank my family for supporting me unconditionally. My parents never criticize me on my decisions and are there for me if I do make mistakes. Their pride in me is one of the main factors that drives me to excel at whatever I do. My father has always been a role model for me with his imagination, sense of humour, and friendly attitude towards all people. My mother has shown me what a person can do if you apply yourself. My brother has also helped me with academic advice and the occasional mathematical direction that 1 need.

It is my belief that who we become depends entirely upon the path by which we get there. Each person mentioned above has played some part in getting me where I am today. To each I am eternally grateful. 


\section{Table of Contents}

Acceptance Sheet

PAGE

Abstract

ii

Acknowledgments

iii

Table of Contents

iv

List of Tables

vi

List of Figures

viii

Nomenclature

ix

xii

Chapter 1: Introduction 1

1.1 Control 2

1.2 Impedance Control 4

1.3 Prosthetics 6

1.4 Overview 8

Chapter 2: Analysis of Technologies

2.1 Robotics

2.1.1 Development of Impedance Control

2.1.2 Implementation of Impedance Control 17

2.2 Prosthetics

2.2.1 Current Research

2.3 Natural Motion

2.4 Conceptualization

Chapter 3: System Model

3.1 Design Model

3.1.1 Sum and Difference Model

3.1.2 Stiffness and Position Model 43

3.1.3 Mechanics $\quad 48$

Chapter 4: System Performance 51

4.1 Selection of Spring Functions 51

4.1.1 Stiffness Function $\quad 52$ 
4.1.2 Position Function

4.1.3 Force Function 63

4.2 Geometric Limitations 71

4.2.1 Spring Limitations 71

4.2.2 Torque Limitations $\quad 78$

$\begin{array}{ll}\text { 4.3 Non-Linear Stiffness } & 87\end{array}$

4.3.1 Stiffness Function $\quad 87$

4.4 General Limitations $\quad 99$

4.5 Power and Energy Requirements 109

4.5.1 Motion Energy $\quad 110$

4.5.2 Stiffiness Energy and Power 111

4.5.3 Power: Relation to Joint Stiffness Emor 113

4.5.4 Neutral Force Configuration 118

$\begin{array}{ll}\text { Chapter 5: Implementation } & 120\end{array}$

5.1 Force Generation in Rolamitss $\quad 122$

5.2 Quadratic Spring Design 124

5.3 Limitations of Rolamite $\quad 125$

Chapter 6: Application of Design 131

6.1 Selection of Parameters 131

6.2 Simulation 139

6.2.1 Configurations Definitions $\quad 140$

6.2.2 Task Definitions 145

6.2.3 Simulation Results $\quad 148$

6.2.4 Summary of Simulation Results $\quad 177$

Chapter 7: Conclusions and Recommendations 179

$\begin{array}{ll}7.1 \text { Conclusions } & 179\end{array}$

7.2 Recommendations 186

$\begin{array}{ll}\text { References } & 188\end{array}$

Appendix A: Altemate Proof of Quadratic Springs 197

Appendix B: State Space Representation of Configurations 198

Appendix C: MATLAB Simulation Code $\quad 202$ 
List of Tables

TABLE DEscription

3A Parameter and variable definitions

3B Definition of stiffness and position contributions (unloaded arm)

GA

GB

bC
Parameter selection

Chosen parameters

Energy Expenditure of Simulated Tasks
Page

37

44

134

142

177

viii 


\section{List of Figures}

Figure DEscritrion

Page

3.1

Junctions

34

3.2

Basic Actuator Configurations

37

3.3 Bond Graph of System

38

3.4

Change of Inputs

38

3.5

Manipulation of Bond Graph

41

3.6

Separation of Components

41

3.7 Combination into Stiffness and Damping Fields

42

3.8

System Inputs

43

3.9 Stiffness and Position Contributions to Input $x_{1 .}$

44

3.10 Inputs as Sum of Stiffness and Position Contributions

46

3.11 Bond Graph Representation Using Stiffness and Position Inputs

47

3.12 Sum of Joint Torques in Equilibrium

48

4.1

Arbitrary Stiffness Functions

54

4.2

Linear Stiffness Functions

54

4.3

Spring Force

64

4.4

Operation of the Arm

69

4.5

Definition of Spring Stretch Limitations

72

4.6

Identical Dual Actuator Configuration

73

4.7

Stiffness and Position Configuration at Rest

75

4.8

Spring Limits

77

4.9 Determining the Smaller of Twu Values 79

4.10 Limiting Torque 85

$4.11 \quad$ Variable Stiffiness Operation 86 


\section{List of Figures (continued)}

Figure

DESCRIPTION

PaGe

4.12 Spring Stiffness Functions that Change Joint Stiffiness

by $\pm 5 \%$ and $\pm 100 \%$ for a 90 degree Endpoint Deflection.

98

4.13 Typical Stiffiness Element Force-Deflection Curve

101

4.14

Positive Limiting Torque

103

4.15 Variable Stiffness Limitations

4.16 Spring Force Function That Results in a Large Stiffness

Controlled Region

4.17 Corresponding Stiffness Controlled Region for Figure 4.16

4.18 Approximate Stiffness Controlled Region using Stiffness Elements

Defined by Figure 4.13.

Ideal Force Curve for Minimizing Power

Comparison of Force Functions

Power Savings Over Quadratic Equation

Neutral Stiffness Force Configuration

Basic Rolamite Configuration

Quadratic Spring Rollerband Shapes

Maximum Stress as a Function of Band Thickness 


\section{List of Figures (continued)}

FIGURE DESCRIPTION

PAGE

$\begin{array}{lll}6.5 & \text { Configuration } 1 & 141\end{array}$

$\begin{array}{lll}6.6 & \text { Configuration } 2 & 142\end{array}$

$\begin{array}{lll}6.7 & \text { Configuration } 3 & 144\end{array}$

6.8 Task 1, Configuration 1 Results 152

6.9 Task 1, Configuration 2 Results 153

6.10 Task 1, Configuration 3 Results 154

6.11 Task 1, Configuration 4 Results 155

6.12 Task 2, Configuration 1 Results 158

6.13 Task 2, Configuration 2 Results 159

6.14 Task 2, Configuration 3 Results 160

6.15 Task 2, Configuration 4 Results 161

6.16 Task 3, Configuration 1 Results 164

6.17 Task 3, Configuration 2 Results 165

6.18 Task 3, Configuration 3 Results 166

6.19 Task 3, Configuration 4 Results 167

6.20 Task 4, Configuration 1 Results 173

6.21 Task 4, Configuration 2 Results 174

6.22 Task 4, Configuration 3 Results 175

6.23 Task 4, Configuration 4 Results 176 


\section{Nomenclature}

SYMBol DEsCRIPTION

b common damping coefficient for identical branches

$b_{1}, b_{2} \quad$ damping coefficients, branches 1 and 2

d rolamite soller diameter

E modulus of elasticity

$E_{1}, E_{2} \quad$ energy exp.nditure, branches 1 and 2

$F$ force

$F_{1}, F_{2} \quad$ force on joint radius, branches 1 and 2

$F_{b 1}, F_{b 2} \quad$ force in damper, branches 1 and 2

$F_{01}, F_{22} \quad$ force on actuator, branches 1 and 2

$F_{\text {in }}$

$F_{k 1}, F_{k 2}$ external forces from weight or loading

force in springs, branch 1 and 2

maximum force that can be produced by spring

minimum (maximum negative) force that can be produced by spring range of force that can be produced by spring

geometric factor of rolamites, combination of length, width, and height

area moment of inertia

combined rotational inertia about joint

spring stiffness, branches 1 and 2

maximum spring stiffness, branches 1 and 2

minimum spring stiffness, branches 1 and 2

common maximum stiffness for identical branches

common minimum stiffness for identical branches

effective joint stiffness

modeled environment stiffness

max: vum joint stiffness

minimum juint stiffness

set joint stiffness

difference between actual and set joint stiffnesses

maximum space for joint actuation system

minimum length of spring (at maximum negative displacement)

limiting length of branch operation, $I_{\mathrm{lm}}=I_{\max }-I_{\operatorname{man}}$

rest length of spring

length of forearm

mass of arm

power savings index

maximum power savings (decimal percent)

angular momentum of arm

power requirements, branches 1 and 2

radius of rolamite roller 


\section{Nomenclature (continued)}

\section{SYMBol Description}

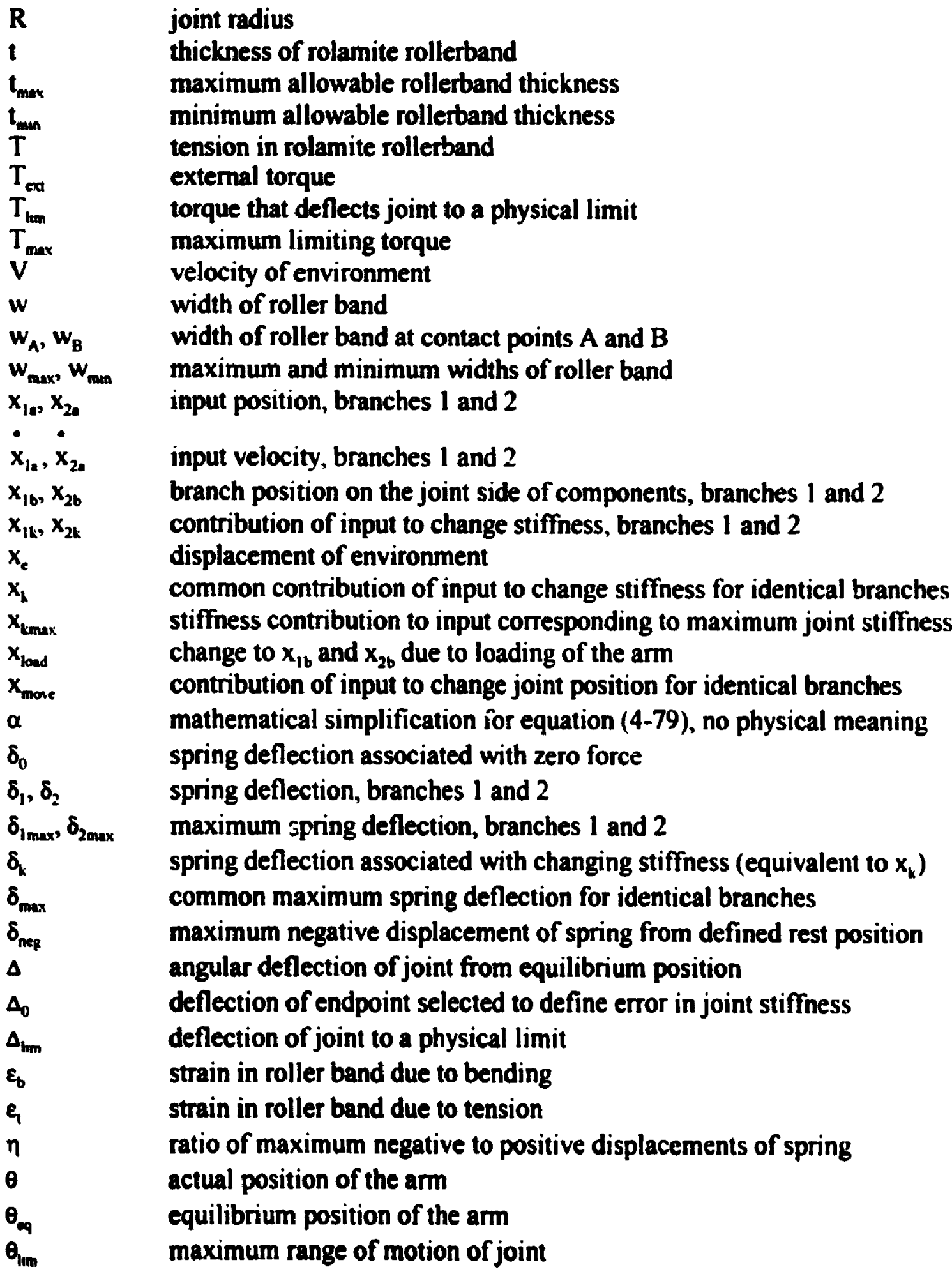




\section{Nomenclature (continued)}

SYMBol Description

$\lambda$

$\boldsymbol{\mu}$

$\xi$

Eo

$\boldsymbol{\sigma}$

$\sigma_{c}$

$\varphi_{\text {mine }}$

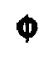

$\phi_{1}, \phi_{2}$

$\phi_{3}$

$\phi_{c}$

$\phi_{e}$.

$\phi_{\mathrm{e}}$

$\phi_{4}$

$\Psi$

$\boldsymbol{\Psi}_{1}, \boldsymbol{\Psi}_{2}$

$\boldsymbol{\psi}_{\mathrm{e}}$.

$\boldsymbol{\psi}_{\mathrm{s}}$ unknown parameter in solution of second order differential equations parameter associated with solutions of differential equations relative error of joint stiffness relative error chosen to define error function of joint stiffness stress in roller band endurance stress of roller band material input parameter to Configurations $\mathbf{2}$ and 3 common spring force function fur identical springs spring force function, branches $I$ and 2

spring force function for third spring in Configuration 3 non-quadratic spring force function non-quadratic spring force function that causes increase in joint stiffness for positive joint deflection (hardening joint spring)

non-quadratic spring force function that causes decrease in joint stiffness for positive joint deflection (softening joint spring) quadratic spring force function common spring stiffness function for identical springs spring stiffness function, branches 1 and 2 non-linear spring stiffness function that causes increase in joint stiffness for positive joint deflection (hardening joint spring) non-linear spring stiffness function that causes decrease in join stiffness for positive joint deflection (softening joint spring) 


\section{Chapter 1}

\section{Introduction}

Mention the word robot to most people and the images that come to mind are the human mimicking machines of science fiction - the R2D2 and C3PO of Siar Hars fame or Robbie the Robot from Forbidden Planet. This view is certainly justifiable considering the origin of the word robot, namely Karel Capek's 1921 play "R.U.R." (Rossum's Universal Robots) using the Czech word for forced labor. It is noteworthy that these artificial humans were chemically created. In fact, Capek argued that metal machines taking the place of humans could never happen. The term came into popular use through the works of science fiction writer Isaac Asimov. Over the years various other technical terms have appeared to describe such beings: automatons, androids, cyborgs (part human, part machine), and more recently, Al's (for artificial intelligence).

Certainly the ability to create such machines is far beyond even today's technology. These days, the term robot more often describes an automated industrial manipulator or small autonomous vehicle. Academically speaking, the study of robots most often refers to the former of these applications. There is some study of autonomous vehicles, especially for exploration of other planets, but typically this is the domain of the 
home hobbyist. However, it is apparent that defining the field of robotics design and analysis is not an easy task. Some people argue that hobby robots are merely toys. Others argue that industrial robots are simply automated machines. Typically the definition of a robot includes the performance of a human-like function by a machine. Sometimes the definition includes the additional criterion that the machine be autonomous, i.e., no input from humans. It is then ambiguous as to whether the field of prosthetics falls within this category. Certainly human-like functions are the main purpose of these machines. However, they do not meet the autonomous requirement, but then neither do some of the landmarks of robotics technology, such as the Canadarm used on the space shuttles which the crew controls via hand controllers.

The purpose of this thesis is to analyze and design a scheme for implementing a variable impedance joint. This work is applicable to robotic manipulators, whether they be for industrial application, medical use, space manipulation, or limb prosthetics. In order to understand the need for this type of research, one must be cognizant of issues prevalent in the fields of robotics and prosthetics.

\subsection{Control}

By far most of the work in robotics research is in the area of control. To many people this calls to mind some sort of joystick or input device. In engineering it usually refers to the transfer function through which the output is controlled. In other words, the controller accepts a commanded motion input and calculates the voltage to apply across a motor or other form of actuator. The importance of the controiler becomes apparent when one adds feedback. The feedback becomes another input into the controller and it recalculates the output voltage based on the commanded motion and feedback, i.e., the 
error is equal to the difference between commanded and measured values. The purpose of the controller is to apply input to the system to drive the error toward zero.

Control schemes differ in input, feedback, and algorithm to calculate the output. Analyzing the implications of each type and developing new control schemes are the main focus of most robotics research. A basic position controller inputs a position change of the manipulator endpoint, calculates necessary changes to each joint of the manipulator to reach that point, and feeds back the actual position in order to make any modifications to the output. Basic force controllers apply the same technique with the exception that they input and feedback desired and actual forces, respectively. Sometimes feedback represents more than one type of information, such as position, velocity, and force. The use of each changes the nature of the controller. How strongly the controller reacts to errors in the actual output depends upon the feedback gain. One would assume that high gain is best since it would eliminate any errors as quickly as possible. In reality this can cause many problems, especially in terms of stability. Choosing an appropriate gain and controller structure are not easy tasks and many consider it more of an art than a science.

A higher level of control is the decision making process that determines the desired motions or forces. In the case of joysticks or prostheses, humans make the decisions. In many industrial applications, such as automotive manufacturing, an operator programs the motions into a computer which "plays back" the same sequence of movements. Often the computer has several stored programs in memory so that the same robot can perform a variety of tasks. The ultimate high level controller for a robot is one that plans the motions for a given task on its own. Although current technology does not permit such an autonomous mechanism, a strong foundation for this sort of machine does exist. Obstacle avoidance techniques require a robot to modify its trajectory based on the sensing of obstacles in its path, including moving invaders. Usually, range sensors detect the location of an obstacle and send this information as feedback. This type of feedback 
differs from the low level controller feedback that compares actual output values with those that are commanded. In this case the feedback is used to change the desired motion itself. The command to change trajectory becomes stronger in proportion to the distance of the obstacle. In this sense the robot makes the decision to deviate from its planned trajectory in order to avoid the obstacle and then calculates a new trajectory based on its new position.

Neural networks allow a computer to learn a motion without the need for direct programming. Typically the robot attempts the motion by trial and error and compares the results with the desired trajectory. By weighting correct movements positively and penalizing incorrect movements, the computer is able to learn the proper trajectory. Expansion of this technique to a desired task rather than a desired motion may allow computers to plan their own methods of completing the desired tasks.

\subsection{Impedance Control}

The design work in this thesis is based around the concept of impedance control. It is therefore important that one understands the nature of impedances. The term impedance is commonly associated with electronic circuits. In that form it refers to the relationship between voltage and current through a component such as a resistor, capacitor, or inductor. In mechanics the term has a similar meaning in that it describes a relationship between force and velocity within a component such as a spring, damper, or inertial mass. In both cases the reverse relationship, or inverse impedance, is known as the admittance.

In particular, the dynamics of a mechanical system are usually described by a set

of equations that relate the forces to the components of motion, i.e., position, velocity, 
and acceleration. Thus the typical dynamic representation of a mass-spring-damper system (Figure 1.1) is

$$
M \ddot{X}+B\left(\dot{X}-\dot{X}_{0}\right)+K\left(X-X_{0}\right)=F
$$

where $\mathbf{M}=$ mass

$B=$ damping coefficient

$\mathrm{K}=$ spring stiffness

$F=$ force felt by environment

$\ddot{X}=$ acceleration of mass

$\dot{X}=$ velocity at given moment

$\dot{X}_{0}=$ commanded velocity

$\mathrm{X}=$ position at given moment

$X_{0}=$ commanded position

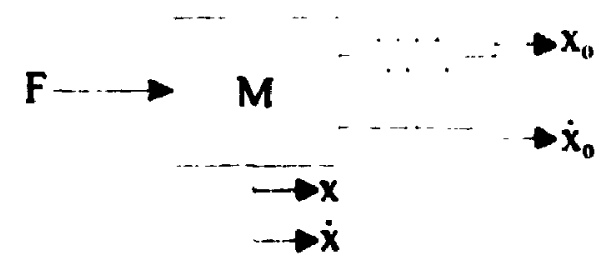

Figure 1 I Mass-spring-damper system

If one controls the motion of the system, the resulting forces are a function of the system impedances. Conversely, if one controls the force then the motion is the result of the impedances. It is impossible to control both motion and force in the same direction. Impedance control recognizes this phenomenon. The purpose of impedance control is to regulate the relationship between force and motion by varying the system impedances.

There are two means for implementing this technique. One can directly vary the impedance properties of the manipulator. This may require complicated devices for 
varying stiffness, damping, and inertia. The second, and more common, implementation uses feedback to simulate and mask system impedances. Position feedback creates forces that are proportional to the difference between commanded and measured positions. This is identical to the properties of a spring where the commanded position is the same as the equilibrium position of the spring. Similarly, velocity feedback simulates a damper. Acceleration feedback could be used to simulate mass effects, but it is more common to use force feedback. The acceleration is proportional to the difference between external and applied forces. The applied force is proportional to the difference between commanded and measured external forces. Combining these relations reveals that the external force is proportional to the manipulator mass and a function of the feedback gain. The gain thus has the effect of masking the mass. Use of feedback can therefore be used to modulate effective stiffness, damping, and apparent mass. The attraction of this type of implementation is the ability to change impedance inside the controller regardless of the physical nature of the manipulator. Such flexibility is difficult, if nut impossible, in mechanical variation of impedances. However, it does have its own limitations, such as efficiency and stability, which will be discussed later. This thesis focuses on the application of mechanical modulation of impedances.

\subsection{Prosthetics}

Engineering by nature involves the application of knowledge. The ultimate goal of engineering is a product that improves some aspect of our lives. The process by which this goal is reached often follows three steps: theoretical analysis, design, and development. Perhaps the largest disappointment to engineers is when the product of their work is not used. It is therefore a matter of concern that developments in upper limb prosthetics have not been well accepted by amputees. Statistics show that only 50 
percent of arm amputees use a prosthesis, and 90 percent of these are passive cableoperated devices. The remaining 10 percent use powered devices [LeBlanc, 1987].

Prosthesis refers to any artificial device that attempts to replace some natural function within the human body. This thesis focuses on issues that are important for use in artificial joints, particularly those of the upper limbs. Throughout the thesis the terms upper limb prosthesis and prosthesis are used interchangeably.

By far, the most common types of upper limb elbow prostheses in use today are based on cable-operated devices developed shortly after the Second World War. There are a variety of externally powered prostheses available that are based on technology developed in the 1960s and 1970s. However, these devices are based around the nature of the input signal and not on the mechanics of human motion. The motion is derived from techuiques developed by the robotic industry. Thus the resulting prostheses perform more like robot manipulators than human arms. This is believed to be one of the main reasons why amputees do not favorably receive such devices. Human motion is neither easy to analyze nor emulate because of its complexity.

Common complaints from amputees regarding available prostheses include poor performance, substandard cosmesis, and they just do not look, feel, move, or work like intact human arms. Cosmesis aside, the complaints can be summarized by one statement: existing prostheses simply do not adequately mimic the behavior of natural arm motion in constrained or unconstrained movements. The history of prosthesis development is particularly lacking in the analysis of constrained motions. Contact tasks are a regular part of people's daily routine and define the limits of human abilities. Unconstrained movements, such as pointing and swinging the arm, place requirements only upon the appearance of the motion and not on interaction with the environment. Dealing with contact issues is much more complicated and less understood. In terms of prosthetic application, the work performed in this thesis attempts to redesign the nature of the prosthesis in order to achieve more natural motion. 
1.4 Overview

Chapter 1 introduces the purpose and concepts of the thesis. A background analysis of robotic and prosthetic technologies, as well as the current understanding of natural motion, are presented in Chapter 2 . Included is a study of perceived problems in each field and a proposed solution. A model definition for the proposed design is developed in Chapter 3. Chapter 4 presents an analysis of the model performance in terms of stiffness, forces, limitations, effects of non-ideal components, and power and energy requirements. Practical implementation of the design requires a method of physically realizing the required components. Chapter 5 offers such a practical implementation using rolamite devices. Simulations based on the application of the design to a prosthesis are presented in Chapter 6. A discussion of implications and conclusions follows in Chapter 7. It also includes an outline of recommendations for future work.

8 


\section{Chapter 2}

\section{Analysis of Technologies}

The objective of this thesis is to develop a manipulator design, with emphasis on prosthetic application, that improves performance over existing devices. The performance is judged on a variety of factors that include power and energy savings, low internal forces, manufacturability, practicality, and stability. In terms of prosthesis performance, improvements are judged on the same criteria, but more importantly on the ability for motion to appear natural and the reduction of the forces transmitted to the amputee's stump.

Improving the performance requires an understanding of robotic and prosthetic technologies as well as the mechanics of human arm motion. In this chapter, each is examined in terms of the history, present state, current issues, and direction of the technology. Although each field is presented independently, there are overlapping concepts. Included are problems associated with the currently available manipulators and prostheses. In response to these problems, a plausible solution is developed using available technology and the understanding of human motion mechanics. The main 
focus of this thesis is the development of the solution from concept to proposed implementation, including limitations of the design.

\subsection{Robotics}

The origins of robot control date back to mechanical master-slave manipulators created for radioactive lab work in the 1940s and 50s [Whitney, 1987]. The operator controlled the position and velocity directly and felt the contact forces through the manipulator. Descendants of these devices using electric servo mechanisms maintained this force reflection relationship.

The master-slave architecture was expanded in the 1960s for use in forcefeedback artificial elbows for amputees [Rothchild and Mann, 1966]. This implementation used signals from muscle electrodes as the control inputs. Time delays caused the elbows to become unstable. At the time, force-feedback was also proposed for use in space manipulators that would be controlled from ground based stations. Similar delays due to coding, decoding, error checking, and channel sharing caused the demise of this application. Delays due to dynamic bandwith limitations and computation time still plague such systems today [Eppinger \& Seering, 1986, 1989; Hogan, 1988; Colgate \& Hogan, 1989; Colgate, 1989; Paul, 1987; Whitney, 1987; Lu \& Goldenberg, 1992].

Up to this point in time, performance of tasks had been controlled by a human operator. With the emergence of computer control it became necessary to determine the issues that must be considered in performing a task. These included such things as trajectory planning, positioning, stability, and interaction with the workspace. This lead to a growth in different control concepts [Whitney, 1987]. Such concepts are listed below with a brief description of the technique. 


\section{Explicit force control [Nevins \& Whitney, 1973]}

Force is directly commanded and measured. The goal is for the output force to follow the input as closely as possible.

\section{Accommodation [Whitney, 1977]}

This is also know as damping control. Contact forces are sensed and are multiplied by an admittance matrix (velocity/force) to modify commanded velocities.

3. Passive compliance [Watson, 1976; Drake \& Sumunovic, 1977] Compliant elements (springs) are placed in the wrist or joints. This minimizes contact forces by allowing the workpiece to control the exact location of the end-effector. Remote Center Compliance (RCC) [Drake \& Sumunovic, 1977] has been proven to be very successful for peg-in-hole tasks.

4. Stiffness control [Salisbury; 1980; Salisbury \& Craig, 1982]

Motion is controlled by commanaing position. Sensed forces are converted to position modifications via a stiffness matrix to produce the desired forces.

5. Hybrid force-position control [Mason, 1981; Railbert \& Craig, 1981]. Force is controlled in directions of contact and position is controlled in directions of free motion.

\section{Impedance control [Hogan, 1980a, 1985a,b,c]}

This control scheme is based on the fact that one cannot control both force and motion in the same direction. It is difficult then to guarentee stability for both position and force. Modulating impedances controls the relationship between force and motion. This can 
allow the indirect control of one property (force or position) while directly controlling the other. Thus constrained or unconstrained motion can be controlled with a unified approach rather than using separate control techniques.

Impedance control essentially combines and expands upon stiffness and damping control. Hybrid control inclucies force control when constrained. Passive compliance is not really a control scheme but a mechanical adaptation of basic position control. Therefore the state of robotics technology for performing both contact and free motion essentially leads to impedance and hybrid control schemes.

Hybrid control divides the task space into two orthogonal subspaces. Force is controlled in all directions that do not permit arbitrary motion, and position is controlled in all other directions. It is essentially a combination of position and explicit force control. This type of scheme gives rise to an inherent instability at the point where contact is made. Here it intermittently switches between control schemes. Similar arguments can be made about any force feedback control scheme. Stability problems tend to originate when the manipulator makes contact, thus changing the controller performance [Whitney, 1977; Paul, 1987; Hogan, 1988]. Analysis shows that instability is an unavoidable consequence of closing a force feedback loop around a nonbackdrivable robot [Colgate, 1989].

Backdrivability does allow for control of force characteristics through torque control. However, this creates a variety of additional problems. It has been suggested that backdrivability is not desirable for several reasons:

(1) It requires energy to simply hold an object against gravity

(2) Implementing backdrivability is cifficult.

(3) Direct drive robots require lightweight, and thus high speed, motors that I ually require gearing dr!m. The friction torque between the gears tends to increase as the square of the gear ratio, thus the required torque to backdrive the motor includes a large 
friction torque. As well, motor and amplifier non-linearities tend to be a problem. Backdrivability is associated with efficiency, and the reader is reffered to [Mansfield. 1988] for more detail.

While most work in hybrid control deals with higher level supervisory and planning designs, lower level considerations, such as stability and bandwidth limitations, are important issues. Stability analysis of force feedback control [Whitney, 1977; Hirzinger, 1983; Roberts et al., 1985; Wlassich, 1986; Eppinger \& Seering, 1986, 1987; Kazerooni, 1987] shows that there is a tradeoff between high bandwidth and the combined stiffness of the sensor and environment. This means high bandwidth force control requires a compliant sensor or environment. Force control is therefore not suited for manipulation in unknown environments.

Impedance control, on the other hand, uses one scheme to control position and force. The goal of impedance control is to provide compliance in the event of uncertainties. No attempt is made to follow a commanded force trajectory. Position is commanded in task space [Lu \& Goldenberg, 1992]. When no constraint is made on the motion, the trajectories in task space and real space are identical. When in contact, the commanded trajectory exists only in task space. Adjusting impedance creates the desired force response [Field \& Stepenenko, 1992]. Thus impedance control can also be viewed as a combination of position and force control, but using a single strategy unlike the dual control scheme of hybrid force-position control.

\subsubsection{Development of Impedance Control}

Impedance control was the culmination of work by Hogan on prosthetic control and antagonistic actuation [Hogan, 1980a,b, 1984a]. This work lead to the introduction of impedance control in robotics [Hogan, 1984b, 1985a,b,c]. It built on concepts that 
originated in stiffiness control [Salisbury, 1980] and damping control [Whitney, 1977], but Hogan noted that no physically realizable system could eliminate inertial effects. By adding an inertia term one could modify the apparent mass from the view of the endeffector.

The motivation behind impedance control is the realization that one cannot control both position and force in a given direction. For instance, to apply a force to a surface one commands a motion until the response force is equal to the desired value. This is also true for dynamic events.

Subsequent work has revealed that impedance control provides a solution for many problems associated with robotics. Each of these problems is discussed below.

1. The majority of control schemes are designed around specific tasks, such as free motion or creation of static contact forces (e.g., RCC). Application of these schemes to a variety of tasks results in switching between control techniques as in hybrid control. Impedance control allows for a uniform approach to manipulation, including analysis of free motion, constrained motion, obstacle avoidance, and dynamic interaction [Andrews \& Hogan, 1983; Hogan, 1985b, 1988].

2. It has been recognized that some form of compliance is necessary for assembly and tool use [Whitney, 1977; Peshkin, 1990; Schimmels \& Peshkin, 1991]. Impedance control includes the modulation of compliance (inverse of stiffness) so successful compliance techniques, such as RCC, can be incorporated within its framework.

3. A common control problem for contact tasks is how to describe the robot-en's ironment dynamics and create a control law that stabilizes both position and interactive force [Katic \& Vukobratovic, 1994]. Stability problems limit most force feedback methods. Studies show that impedance control can regulate force without the need for force 
feedback [Hogan, 1985a,b]. In fact, stability can be guaranteed even in the event of uncertain surroundings so long as the environment is stable and passive [Hogan, 1987. 1988] and the commanded joint stiffness remains within limits placed upon it by natural joint flexibility [Latre \& Cabrera, 1991]. As well, it is possible to design the controller such that stability is maintained in the presence of high frequency unmodeled dynamics [Kazerooni et al., 1986a,b; Kazerooni, 1987].

4. Most control studies concentrate on motion in space and do not include contact situations [Katic \& Vukobratovic, 1994; Whitney, 1987]. Interaction analysis is necessary for modeling everyday occurrences and tasks. Basic position and force control do not deal with this dynamic interaction [Hogan, 1985a]. Few research studies account for rigid body dynamics of a robot in hybrid control [Khatib, 1986, 1987]. These studies focus on strategy while dealing with control only in passing. Even those that do include contact generally model interaction with the environment as the deformation of some elastic element [Whitney, 1987] or as small changes in system parameters [Hogan, 1988] More general models are available. However, the elastic deformation model alone makes it clear that control of robots in contact with the environment requires the management of at least compliances. A more accurate model includes inertias. Impedance control deals with the regulation of both compliances (inverse of stiffness) and inertia and accounts for the dynamics of interaction. As previously mentioned, impedance control also has the ability to guarantee stability in spite of poor environment modeling.

5. Using impedance control can circumvent the inverse kinematics problem [Hogan, 1985b, 1987]. Geometry defines the relation between the endpoint and the joint angles. However, given the desired position of the endpoint it is often difficult to calculate what joint angles are appropriate to reach it. Often there is more than one configuration that 
will produce the same position and orientation of the endpoint. Points that allow an infinite number of solutions are known as redundancies, as there is a redundant degree of freedom. Usually this occurs when the rotational axes of two joints are in alignment. As well, there are locations for which a degree of freedom is lost, usually on the boundary of the workspace, which are known as sigularities. Typically, the design of a robot controller requires that it does not allow the joints to pass through these singularities, as with the Canadarm used on the space shuttles. This sometimes produces odd and unexpected motions as the arm moves to avoid these singularities.

6. Perhaps the greatest danger that robots impose is the possibility of colliding with obstacles, especially people. Impedance control offers a two-fold solution to this problem. First, impedance control supplies a framework for implementing obstacle avoidance, including moving obstacles [Andrews \& Hogan, 1983; Hogan, 1985c]. In addition, the impedances can be set low when collision is likely or imminent such that contact does not result in large forces on the ubstacle or person.

In addition to the solutions that impedance control offers, Hogan and others argue that any feedback controller does not only regulate a variable such as position or force, it also changes the dynamic behavior of the system [Wlassich, 1986; Hogan, 1987; Colgate, 1988]. In fact, force feedback is equivalent to the modulation of the manipulator's effective inertia as seen by the environment. Volpe shows an equivalence between second order impedance control (i.e., inertia included) and proportional gain explicit force control with feedforward [Volpe, 1990; Volpe \& Khosla, 1991, 1993]. While the above arguments are opposite in view (Hogan implies force-feedback is poorly regulated impedance control while Volpe argues that impedance control is cumbersome and limited force control), they do suggest a relationship between the two schemes. However, the results of using such schemes show that they are not identical with respect 
to performance. Since Volpe's arguments against impedance control tend toward specific application, proper implementation may avoid such perceived problems.

\subsubsection{Implementation of Impedance Control}

\section{ACTIVE IMPEDANCE CONTROL}

The traditional implementation of impedance control consists of dc-motor driven joints with the commanded joint torques calculated using desired impedance properties and feedback. Position feedback translates to stiffness control, velocity feedback controls daniping, and force feedback controls the apparent mass. In such an implementation the desired impedances exist only within the control software; they do not exist as physical properties of the system but appear so from the point of view of the environment [Sharon et al., 1989; Latre \& Cabrera, 1991; Katic \& Vukobratovic, 1994]. This type of implementation is best termed active impedance control since it requires an input of energy to simulate the storage and dissipation of energy in stiffness, damping, and inertial elements. Problems with active impedance control consist mainly of stability issues. Stability can be guaranteed under certain conditions, however it is dependent upon thie configuration [Eppinger \& Seering, 1986, 1989; Kazerooni, 1987; Whitney, 1987; Colgate \& Hogan, 1989; Colgate, 1989; Volpe \& Khosla, 1991, 1993; Lu \& Goldenberg, 1992]. Another drawback to active control is that the physical characteristics of the actuators, joints, and manipulator links can limit performance [Lawrence, 1989; Latre \& Cabrera, 1991]. 


\section{PASSIVE IMPEDANCE CONTROL}

An altemative implementation to active impedance control is the mechanical design of variable stiffness, damping, and mass in the manipulator itself. This type is best termed passive impedance control since the impedance elements work passively with the environment. External energy is only required to change the impedance properties and in the execution of a motion. Passive actuation offers some improved characteristics over active control. The use of passive elements can guarantee stability in open loop control since they do not require feedback [Hogan, 1984a; Sugano et al., 1992; Mittal et al., 1993]. Improved open loop control puts fewer restrictions upon the controller in terms of stability and computation. Manipulator forces are the reaction of physical elements rather than the result of computations within the controller. This speeds up computation time and allows the controller to focus on higher order operations. As well, feedback may not be always desirable. Time lag and computation time can cause instabilities. Even with faster computation and communication, fast interaction may be required beyond the limits of ideal instantaneous feedback. For instance, feedback in primates is far too slow to compensate for environmental dynamics, yet they are experts of dynamic interaction [Hogan, 1985b]. Passive impedance control also improves energy efficiency. Forces are created by passive elements, such as the deflection of springs, and therefore do not require a constant energy drain to create constant forces. Active control, on the other hand, requires constant energy drain to produce forces via an actuator.

Passive impedance control is not without its drawbacks though. Using passive components can guarantee stability but they are typically not very programmable. Remote Center Compliance (RCC) [Nevins \& Whitney, 1973] is an example of this. Designing variable components is difficult and results in complicated, bulky mechanisms. Modulation of impedances by mechanical means requires redundant actuators [Russell \& English, 1995]. Following the laws of physics, variation of mass is 
not possible without nuclear reaction. However, moving the center of mass can modify the rotational inertia of a manipulator. Controlled variable damping research is rare [Laurin-Kovitz et al., 1991]. The bulk of passive impedance control work deals with variable stiffness elements.

Actuation research provides a variety of methods for controlling end-effector compliance. Cutkosky and Wright [1986] expand on the passive Remote Center Compliance (RCC) idea by extending the number of compliance centers using pressure controlled fluid filled bladders. Kaneko et al. [1988] propose Direct Compliance Control (DCC) whereby directly tuning the stiffness of each joint regulates the compliance of the end-effector. A variety of variable stiffness actuators using antagonistic actuation have emerged, including hydraulic cylinders [Goswami et al., 1990], fluid-filled bladders [Cutkosky \& Wright, 1986], pneumatic bladders [Immega, 1987; Mills, 1990], and nonlinear springs [Yi \& Freeman, 1992; Laurin-Kovitz et al., 1991; Russell \& English, 1995]. However, in most schemes of this sort the modulation of stiffness and position are coupled. This requires feedback to control the stiffness as a function of joint displacement. Mittal et al. [1993] introduce an actuator with only minor stiffness and position coupling within applied torque limitations. Another solution uses quadratic springs (force proportional to square of deflection) [Laurin-Kovitz et al., 1991; Russell \& English, 1995].

The motivation behind antagonistic actuation is the use of solutions found in nature [Hogan, 1980a, 1984a; Tong \& Somerset, 1985]. It is the method by which most higher animals regulate stiffness and motion. They use non-linear stiffness elements (muscles) in antagonistic muscle pairs. Activation of the pairs in the same direction, termed co-activation, increases stiffness while differential operation causes motion. Redundant actuation is the key to mechanically varying impedance [Towhidkhah et al., 1993]. Skeletal muscles greatly outnumber joints in animals. Even the simple human elbow has four actuators. Antagonistic actuation is ideal for use with cable operated 
manipulation [Jacobsen et al., 1984, 1986, 1989; Ma et al., 1992; Lee et al., 1993] since cable, like muscle, can only supply force in one direction.

\subsection{Prosthetics}

A detailed history of prosthesis development is beyond the scope of this thesis. Robert Mann presents an excellent review in his 1981 report on Cybernetic Limb Prosthesis [Mann, 1981]. Presented here is a summary of the history presented in that report.

The beginnings of upper limb prosthetics date back to the "hook". Early advancements in prosthetic technology have a correlation with major wars due to the influx of wartime amputees. The American Civil War saw the creation of various terminal devices (eating utensils, specialized hooks) attached to the amputee via a leather socket. Cable-operated devices controlling the elbow and hand were introduced shortly after World War Il in response to the large number of upper-extremity amputee veterans. A harness worn around the shoulders held the socket in place. For below elbow amputees a springloaded hook-like terminal device was operated by rounding the shoulders, thus shortening the operating cable. The same maneuver performed two functions for an above elbow amputee: opening the terminal device or flexing the elbow, depending on whether the elbow joint was locked or unlocked by another cable.

On the basis of work he began in 1948, Norbert Wiener brought about the idea in the early 1960s of using the natural signals from the brain to control an artificial limb replacement [Wiener, 1961]. In this manner the signals intended for control of the natural limb would be interpreted and used to produce corresponding motion of the prosthesis. Likewise, the prosthesis would be able to create feedback signals appropriate for sensed phenomena and send them back into the central nervous system. In theory this 
implementation would require no retraining of the amputee since natural signals and feedback would be used to control the prosthesis. Wiener adopted the term cybernetics, from the Greek for steersman, for this type of man-machine interface.

By this time there had already been work performed using myoelectric signals (MES) for control of prosthetic hands in Germany and Russia. Although measurement of MES signals by surface electrodes was primitive compared to Wiener's concepts, it was practical within the bounds of technology and the understanding of CNS and muscle signals. At about the same time, the Boston Elbow project was underway [Williams, 1986]. This was the first atrampt at translating MES signals into natural motion commands to a prosthesis. Bioelectric signals from the bicep and tricep muscles were used as an indication of activation level to control the velocity of the arm. The Boston Elbow was also the first myoelectrically controlled prosthesis to provide some force feedback to the controller. External forces were measured and subtracted from the motor applied forces so that the amputee had to supply a constant muscular effort to maintain a constant force.

The next advancements in prosthetics were in the area of electromyographic (EMG) and MES signal interpretation. In the mid-1970s, the Temple Arm project used myoelectric activity from chest and back muscles as an indicator of elbow, wrist, and hand activation. The MES patterns were processed by an electronic weighting circuit that drove velocity servos at the elbow, wrist, and hand. The Temple Arm did show some success in coordinating elbow and wrist control. However, proper control of the hand was not achieved so they resorted to using MES signals from unrelated muscles. Successful hand control was achieved, however, by the Swedish Hund. This forearm prosthesis permitted wrist motion, finger flexion, and thumb rotation. It employed multiple electrodes on the forearm stump that detected separable patterns associated with each motion. Because the signals were measured at the forearm level, application could not be made to an above elbow prosthesis. 
Analytically rigorous muscle modeling by Jacobsen and Mann [1973] at M.I.T. lead to the development of the Utah Arm [Jacobsen et al., 1982]. The EMG activity of numerous muscle groups in the shoulder girdle was related to torques in forearm rotation, elbow flexion, and wrist flexion. Measurements of EMG from nine to twelve sites on the chest and back were inputs to a computer model that generated torque predictions for the arm joints. The results showed excellent correlation between computer predictions and direct measurement of shoulder and elbow torques. The Utah Arm showed improvements in dynamic response, smoothness, quietness, and weight.

To date the Boston Elbow and Utah Arm still represent the state-of-the-art in upper limb prostheses [Abul-Haj \& Hogan, 1990a,b]. However, the vast majority of upper limb amputees do not use such devices. Only 5 percent of arm amputees use an externally powered prosthesis [LeBlanc, 1987]. An explanation for this phenomenon may be apparent from a critical review of the available prostheses.

Cable-operated devices have changed little since the Second World War. They do permit some functions of normal arm usage, such as holding, but greatly limit the restoration of most natural arm functions. There are several advantages to cable-operated prostheses over externally powered devices. First, the natural activation of an intact muscle is typically used to control the motion of the prosthesis. Once the amputee learns this control, the prosthesis can effectively become an extension of the body and therefore require little concentration. Such connection also allows direct force feedback to the muscles of the amputee. This extension of the body's natural physiological control in this manner is referred to as Extended Physiological Proprioception (EPP) [Childress, 1989]. As well, the devices are not constrained to low velocities or torques. Often the amputee can create fast, fluid motions with practise. In addition, the mechanics of these devices are relatively simple and often can be repaired by the user. There are, however, some disadvantages of such devices. First of all, leaming control of the device requires much concentration on the task since non-relevant muscle groups control the motion. The 
appearance, operation, and motion of the prosthesis are unnatural and noncosmetic. Secondly, it only offers control of either the elbow or hook device at a given time. Coordinated movements using both devices are not permitted. There is no direct control of any aspect of the motion such as speed, trajectory, force, or positioning. Thirdly, constraints are placed on the motion of other parts of the body, mainly shoulder motions, and thus create an additional handicap.

The development of cybemetic prostheses using myoelectric signals as control inputs provides several solutions. Relevant muscle groups control the motion of the devices, thus learning can be more natural and additional restrictions are not placed on other areas of the intact body [Mann, 1981]. Moreover, an attempt is made to properly translate the MES (or EMG) signals into the desired motions. The underlying idea behind such work is that control of the prosthesis should require less concentration on the part of the amputee if it is controlled by the same muscle signals as an intact limb [Mann, 1981].

However, amputee complaints of such devices include poor performance, substandard cosmesis, and they just don't look, feel, move, or work like intact human arms. They provide some useful function, but are limited in effectiveness. Even with MES (or EMG) signals translated properly, the resulting motions are velocity controlled and appear more robot-like than human. The prostheses are able to lift relatively light objects but even simple coordinated movement requires the intact arm to provide the bulk of the coordination. As well, response to disturbances is poor. When not being flexed or extended, the elbow usually locks mechanically to conserve battery power. The only safety in such a case is the elbow shear pin that breaks before excessive force is transmitted to the amputee. In addition, since cybernetic prostheses are externally powered, the motion is not intuitive and requires much concentration. Amputees are better able to predict the simple motion of a cable-operated arm and receive direct force 
feedback through the shoulder hamess. This is the most likely explanation for their choice of the simpler cable-operated prosthesis, or no prosthesis at all.

\subsubsection{Current Research}

In his $1987 \mathrm{Ph} . \mathrm{D}$. thesis [Abul-Haj, 1987], Abul-Haj proposes that there are three problems with current powered prostheses: (1) myoelectric signal acquisition, (2) myoelectric control algorithms, and (3) socket and hamessing. A fourth problem, proposed by Miller [1987], is dynamic behavior. This includes synchronization and inertia effects used in walking, sitting down, opening drawers, climbing stairs, and turning sharply. The use of an arm in these respects is often overlooked. These events can become mental burdens when the weight and dynamic behavior of the prosthesis is not properly matched to the natural arm.

Improvements in the processing of EMG and MES signals are an ongoing subject of study [Parker and Scott, 1986; Hudgins et al., 1993; Latwesen \& Patterson, 1994]. However, solutions to control algorithms and dynamic behavior are the focus of most current research. Collaborative work at M.I.T. shows the results of comparative tests using a prosthesis emulator [Abul-Haj \& Hogan, 19902,b; Popat et al., 1993]. These tests compare the controllers of the Boston Elbow and Utah Arm to a "natural controller" using impedance techniques proposed by Hogan [1985a,b,c]. There are two significant conclusions from these tests. First, impedance control techniques do appear to be matched more closely to natural human arm motions than previous prosthesis control techniques. Secondly, improvements to prostheses that result in more natural motion appear to make powered prosthetics more acceptable to amputees, confirming earlier predictions [Hogan, 1980a, 1982]. Theoretical comparison of the prosthesis controllers also shows that impedance control may offer up to a twenty-fold saving in energy 
expenditure as well as reduced effort on the part of the amputee and lower forces placed on the stump [Russell, 1993].

Another area of research is the actuation of joints. Direct drive dc-motors have been the traditional means of actuation for prostheses, with the joint torques determined by the controller. Crude models of natural joint actuation suggest that antagonistic actuation using non-linear stiffness elements (springs) allows the amputee to vary the stiffness as in an impedance controller [Hogan, 1980, 1982; Russell \& English, 1995], and provides good response to disturbances [Towhidkhah et al., 1993]. Basic muscle models suggest application of this technique can be realized with stiffness elements in series with driving elements, such as dc-motors. However, a variety of alternative actuators have surfaced that combine the driving and stiffness elements as in a natural muscle. Examples include polymer gels [Meghdari et al.. 1993], rubber, collagen, shape memory alloys, piezoelectric polymers and ceramics, magnetostrictive devices, electrostatic motors, and pneumatic bladders [Immega, 1987; Greenhill, 1993]. Many of these novel designs show promise but require much additional engineering and testing before they will come into mainstream use. Hunter and Lafontaine [1992] performed a detailed comparison between many of these actuators and natural muscle.

\subsection{Natural Motion}

It is well established that muscles are more than just generators of force. Certainly human motion control and posture are at least in part a function of muscle and joint impedances [Hogan, 1984; Dolan et al., 1993; Latash \& Zatsiorsky, 1993; Kornecki \& Zschorlich, 1994]. These include the effects of stiffness, damping, and mass on the motion of the arm. Antagonistic muscle pairs about the shoulder and elbow joints control upper arm operations. Co-activation of the muscle pairs allows control of the 
equilibrium position, angular velocity, apparent inertia, and stiffness of the joint [Hogan, 1980, 1984a, 1985d; Winters et al., 1987; Dolan et al., 1993; Lan \& Crago, 1993]. It appears that both negative positional feedback and antagonistic co-activation take part in human posture and motion control [Hogan, 1984a]. Both are sufficient to create the required torsional stiffness, however they are limited in effectiveness. Feedback control performance is bounded by neural transmission delays, typically in the range of $50-100$ milliseconds, and limited bandwidth characteristics of muscles and sensors. Antagonistic co-activation does not require closed loop control and is thus unaffected by neural transmission delays, but at the cost of continuous energy dissipation that performs no mechanical work. The central nervous system (CNS) apparently compromises between the two schemes to achieve an optimal balance.

A number of theories of how the body regulates stiffness have been developed based or: quasi-static experimental studies that consider the stability of an equilibrium point. There are three basic forms for these theories. The equilibrium point - stiffness hypothesis [Polit \& Bizzi, 1979; Hogan, 1980b] states that movement is driven by an equilibrium point that is defined by a function of the difference between the activation of antagonistic muscle pairs. 'The equilibrium point - invariant or $\lambda$-model [Feldman, 1966, 1986] suggests that motion is the result of varying a position offset bias, which creates an equipotential line of solutions based on the external loading on the system (the 'invariant' line). The stiffness regulation theory [Houk, 1979] states that the purpose of reflex mechanisms is to balance responses to transient loading such that the overall stiffness, as seen by the environment, remains approximately constant. This reflex action is necessary due to the non-linear nature of muscle tension that results in a non-linear joint torquedeflection relationship. Detailed analysis of the musculoskeletal system indicates that each of these theo' is does not adequately explain the nature of human motion [Winters et al., 1987]. In particular, the equilibrium point theory, although an appealing concept in open loop control of stiffness and position, has fundamental problems. First, muscles 
do not have the high-stiffness capabilities required to maintain joint stiffness properties as predicted by the theory. Second, co-contraction does not necessarily increase the stiffness in the same manner as suggested. In fact, co-contraction appears to set torque operating ranges for the joint [Winters et al., 1987].

Each of these models hypothesizes about the mode by which motion occurs, however they do not specify a strategy by which a planned trajectory is formed. Studies of trajectory formation in human arm motion fall into two categories: point-to-point and sequential arm movements. Point-to-point movements [Flash \& Hogan, 1985] describe the motion of the arm between two given points in space and analyze trajectory based on that motion only. Sequential arm movements [Takesi \& Honda, 1992] represent a larger class of motion describing a task, such as typewriting, that is a series of movements. Most skilled actions fall into this category. There are three main types of experimentally observed sequential movements in humans: (1) elbow vie-point movements, (2) elbow vie-point with hand to-point movements, and (3) elbow vie-point with elbow to-point movements. Each type represents a series of target motions. Elbow vie-point movements involve the hand moving to a specified target, followed by an elbow motion to the next target, and a final motion of th. hand to a third target. Elbow vie-point with hand to-point follows the same motion with an extra hand target at the end. Elbow viepoint with elbow to-point also follows the same motion with an extra elbow target at the end.

A number of models exist that attempt to explain these motions. The minimum torque-change model [Uno et al., 1989] can predict large horizontal free movements between two targets, whose observed hand-paths are slightly convex. The minimum jerk model [Flash \& Hogan, 1985] always predicts a straight line hand path even in large movements. However, results of the minimum jerk are nearer to the observations in elbow vie-point movements, thus the minimum jerk model is more robust for a variety of tasks. Both models succeed reasonably in describing point-to-point movements but 
neither can accurately predict the trajectory for sequential movements. An optimized time-integral of torque and velocity [Takesi \& Honda, 1992] yields accurate predictions for sequential movements, but fails to explain point-to-point movements. The analysis does show that energy consumption of muscles and motions of the musculoskeletal system are approximately optimum during sequential movements. None of the models are able to provide a general law for human movement control.

An interesting and unique postulate is that fast human arm motions are the result of a learned sequence of events rather than that of planned trajectories and reflex feedback [Gorinevsky, 1993]. There is strong evidence that these fast motions occur in open-loop control since reflex positional feedback has a significant delay and a large gain cannot be achieved. This paradigm lays the importance of fast motions on the memory of the CNS and not in the calculation of trajectories using system dynamics. This is a plausible explanation for the leaming nature of new skills in human. Certainly the dynamics of motion do not change with practice of a new skill. However, performance does improve, suggesting that memory of the sequence of events is vital to the execution of fast motions.

A common method of investigating arm motions is through simulations with muscle models. An advantage of this type of analysis is that it offers access to estimates of internal behavior that is not usually observable. A major problem in experimental analysis of human motion control is the separation of voluntary action, reflex action, and passive properties. Often this requires the subjects to attempt to subdue their instinctive responses to disturbances. Simulations are a tool by which one can point out flaws in past theories, suggest new theories, and suggest new experiments. However, the results are only as accurate as the model. Winters et al. [1987] show that in order to create the dynamic co-activation-impedance relationship apparent in human joints, the model requires three circumstances: (1) there must be at least two muscles surrounding the joint, (2) there must be a contractile element in series with a compliant element, (3) the 
compliant element must be nonlinear, with a concave upward shape (to increase stiffness with co-contraction). In its basic form this model requires a set of antagonistic springs set around the elbow joint [Hogan, 1980a,b, 1984a; Lan \& Crago, 1994]. More accurate joint models include the double joint muscles [Hogan, 1985b; Dolan et al., 1993].

The muscle itself is known to have spring-like behavior [Hogan, 1984a, 1985d; Mussa-Ivaldi et al., 1985], but a simple nonlinear spring does not adequately describe its response. Occasionally a damping element is added in parallel to the spring to represent the muscle's viscous properties. The classic two-element Hill model [Hill, 1938] includes a series elastic component (SEC) and parallel elastic component (PEC) where the PEC is much more compliant. Addition of a viscous element (damper) in series with the SEC completes a three-element model. Latash and Zatsiorsky [1993] point out that stiffness measurements bacod or. the two and three element models produce different results. Winters et al. apply a modified Hill model to the antagonistic actuation of a joint [Winters et al., 1987], resulting in a rigorous eighth-order joint model. Simulation using this model shows that the musculoskeletal system is structurally very different from the simplified quasi-static spring models and parallel impedance models. However, the simplified models do summarize important concepts and help document basic inputoutput behavior.

\subsection{Conceptualization}

The focus of robotic control on constrained motion leads to examination of how natural motion occurs. The conclusions of this examination include the ability of a manipulator to vary its impedance properties. This type of control has the potential to solve many problems of current robotic technology (see Section 2.1.1), such as inverse kinematics [Hogan, 1985b, 1987], contact stability [Hogan, 1987, 1988; Kazerooni et al., 
1986a,b], obstacle avoidance [Andrews \& Hogan, 1983; Hogan, 1985c], dynamic interaction [Hogan, 1985a], and a single robust approach to a variety of manipulation tasks [Hogan, 1985a,b,c]. However, the typical implementation of variable impedance is through active control of joint torque that is calculated by a control algorithm using feedback. Analysis of this approach shows that there are limitations on the achievable impedances based on actuator torque/speed constraints [Lawrence, 1989] and natural impedance characteristics of the manipulator links and joints [Latre \& Cabrera, 1991]. It follows then that such limitations may disappear if the physical impedance properties of the manipulator are varied directly rather than masked by a controller. Better open loop control is often suggested as a method of improving controller performance. The obvious next step is to examine the possibility and consequences of implementing passive variable impedance.

Another view of robotic control is the application of various techniques to prosthetics. The field of prosthetics is arguably an extremu and challenging case of robotic technology application. Common human tasks include interaction with a variety of environments and place a wide range of demands on the control of limbs. This requires limbs that are versatile and adaptable to each situation. Fiostheses that are successful in these tasks and are deemed acceptable by amputees represent robotic manipulators that are robust enough for any application that a human can perform. The oniy limitation of such a design to robotics is the decisinn making process by which the processor controls the manipulator. In the case of prosthetic application, the brain and central nervous system perform these decisions. An exanination of existing upper limb prostheses reveals a number of perceived shortcomings that limit their success. A synopsis of these shortcomings is given below. Note that there are also advantages to each type of prosthesis that are not listed. 


\section{Cable-operated devices}

1. Control requires much concentration on the task.

2. The appearance, operation, and motion of the prosthesis are unnatural.

3. It only offers control of either the elbow or hook device at a given time.

4. There is no direct control over the motion, including speed, trajectory, force, or positioning.

5. Restrictions are placed on the motion of other parts of the body thus creating an additional handicap.

6. Very few normal arm functions are restored.

Veloctty controlled devices (e.g., Boston Elbow and (hah Arm)

1. Motion appears more robot-like than human.

2. There is no direct feedback to the user.

3. External power means the motion is not always predictable or intuitive.

4. Humans are limited in their ability is simultaneously control several degrees of freedom without extensive training and concentration unless the motions correspond to natural movements.

These problems can be summarized into two limitations: (1) control of the prosthesis places an extra burden on the amputee, and (2) the motion is not similar to a natural limb. The first of these limitations requires proper procurement interpretation, and processing of the body's intentions, which is beyond the scope of this thesis. The second limitation requires proper design of the prosthesis so that the interpreted intentions can be executed correctly. It is clear from the study of natural motion (Section 2.3) that this requires the ability to vary impedance properties, particularly the stiffness of the limb. As mentioned above, the next step in implementation of this technique is an analysis of varying impedance properties via passive components. 
The method by which the body varies stiffness is through antagonistic actuation of muscles about a given joint. There is neither a consensus on the mode through which the body does this nor is there agreement on a proper model for muscles in terms of stiffness, damping, and actuation components. A thorough comprehension of these issues is not required, however. It is enough that the body varies the impedance properties. A prosthesis that allows these variations when commanded is all that is necessary. It is the responsibility of the controller to interpret the intention of the body to vary the impedance. The manner in which this occurs within the prosthesis can be designed based on engineering requirements, such as simplicity, rather than duplicating the process by which it normally occurs. In fact, even if the natural modes were understood, the technology does not yet exist to duplicate the complexity of muscles and nerves.

The most important property of variable impedance within the body is joint stiffness. It is responsible for many of the forces created by the body, including posture. Thus it will be the main focus of the design. Damping is also important in terms of motion, although it is less understood. The design must include some form of damping, as with any spring-mass system, to avoid continuous oscillation. The body cannot, however, vary its mass. An apparent mass can be used to mask the inherent properties of the natural inertia, but these effects must be part of an active control scneme that calculates required changes in forces. They cannot be changed mechanically, neither within the body nor within an artificial device. Thus the mechanical design of the manipulator should not include the consideration of variable mass. In summary; the design of a variable impedance joint should include two sets of antagonistic actuators, each consisting of a power source in series with a spring together with enough damping to prevent undue oscillation. 


\section{Chapter 3}

\section{System Model}

Analysis of a dynamic system requires modeling. Often this can take the form of a scaled physical model upon which tests are performed. Usually, the first step is to create a mathematical representation of the system. A variety of methods exist for creating mathematical models. In this thesis, free-body diagrums and bond graphs [Karnopp et al., 1990] are used. Free-body diagrams are common in engineering design and it is assumed that the reader will understand their use. Bond graphs, on the other hand, are not as common and therefore require some explanation.

Bond graphs are based on the principle of conservation of power. At any point in a system the power flowing into that point must be equal to the power flowing out of it. As well, this power must have a direction. For example, power flows from the electrical input of a motor to the mechanical output. A generator has the opposite power flow, from mechanical input to electrical output. Power is represented by different variables in different domains. In mechanical translation, power is represented by the product of instantaneous force and velocity. In rotation it is torque and angular velocity. For electrical systems the power is represented by voltage and current. Hydraulic systems 
represent power using pressure and volume flow rate. These variables are called power variables. When two components (or subsystems) are connected together the power can be represented in the domain of either one. For example, the power transmitted from a motor shaft to a mechanical pump can be represented as the product of shaft torque ard velocity or pump pressure and volume flow rate. Thus the connection of subsystems forces power variables to be equivalent.

The connection of components results in a junction. This is a point where the contribution of one of the power variables from each component adds and the other variable is common. A " 0 " is used to denote common forces, whereas a " 1 " is used to denote common velocities. Examples are shown in Figure 3.1. Note that " $\mathrm{C}$ " is used to represent a spring, whereas " $R$ " represents a damper. Points 1 and 2 share a common force, which is represented by the 0 -junction. The spring and damper share a velocity, namely difference between velocities $v_{1}$ and $v_{2}$. Note that at a junction of only two components, both power variables must be equivalent since the power input and output are equal.

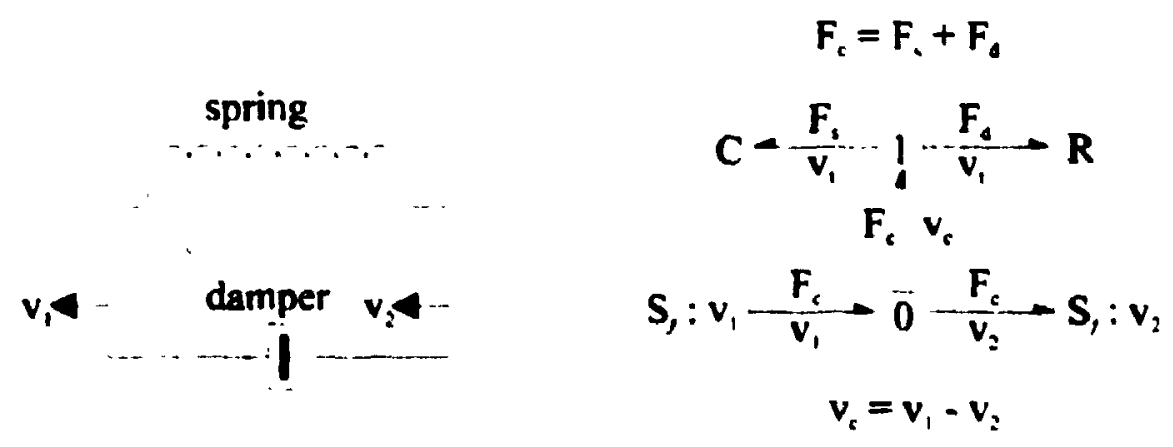

(a)

(b)

Figure 3 1: Junctions (a) system (b) bond graph representation

An important property of bond graphs is the ability to represent different power domains with the same symbols. This is possible due to the nature of power flow. If one 
compares power variables and their relationships with components, an analogy between domains emerges. For instance, the relationship between force and velocity through a damper is given by $F=B \cdot v$. In electronics, a similar relationship exists between voltage and current through a resistor, $\mathbf{V}=\mathbf{R} \cdot \mathbf{i}$. For a spring the relationship involves the integral of velocity (i.e., the position). Likewise, a capacitor involves the integral of current. Derivative relationships exist for inertia and inductors, represented by an "l". Similar comparisons can be made for rotational and hydraulic systems. Thus the bond graph of Figure 3.1(b) can also represent a capacitor and resistor, where force and velocity are replaced by voltage and current. The general representation of the forcing variable is called the effort variable. This includes force, torque, voltage, and pressure in the different domains. The other variable is referred to as the flow variable. This includes velocity, angular velocity, current, and volume flow rate.

Causality is also important in a bond graph. Specifically, one cannot specify both effort and flow of a component. If one specifies the velocity difference across a damper, the force is a result of the damper properties. If one specifies the force then the velocity is the result. The causality is represented on bond graphs by a small line across one end of the bonds. This represents the direction of effort causality. The other end represents the flow causality. In Figure 3.1, the velocity difference is input into the spring and damper, thus flow causality is toward the components. The responses are the forces created, and therefore effort causality is away from the components.

Several additional bond graph components are necessary for the work in this thesis. Amplification or changing domains usually requires multiplication by a constant. For example, the surface force on a gear is multiplied by the radius to calculate the torque. In addition, the surface velocity is divided by the radius to determine the rotational velocity. The element involved in converting or amplifying is called the transformer and is represented by "TF". A similar transformation can occur where the effort in one domain is proportional to the flow in the same or another domain and vice 
versa. For example, the torque of a motor shaft is proportional to the motor current through the torque constant. Likewise the angular velocity is proportional to the voltage via the same constant (though usually represented in reciprocal form as the back-emf constant). The conversion factor in this type of transformation is called a gyrator and is represented by "GY". In addition to system components, there are also inputs and outputs. They are represented as sources and can either be a source of effort, $S_{e}$, or flow, S. The reader should investigate Kamopp et al. [1990] for further understanding of bond graphs.

\subsection{Design Model}

A proposed system for actuation of a stiffness controlled joint is shown in Figure 3.2. Two sets of dampers and non-linear stiffness elements (springs) are connected around the joint. For the purpose of differentiating the sets, the sides are designated as branches. The stiffness and equilibrium position are controlled by the base position of each branch $\left(x_{10}\right.$ and $\left.x_{20}\right)$. Motion occurs by moving them in opposite directions. Pulling on both branches stretches the springs and changes the effective stiffness of the joint. This operation is similar to the antagonistic muscle pairs around the human elbow (as well as many other joints). The environment is represented as a compliant surface with stiffness $K_{e}$. Additional external forces, such as the weight of the arm, are given by $F_{i n}$. Table 3A lists the parameters and variables. 


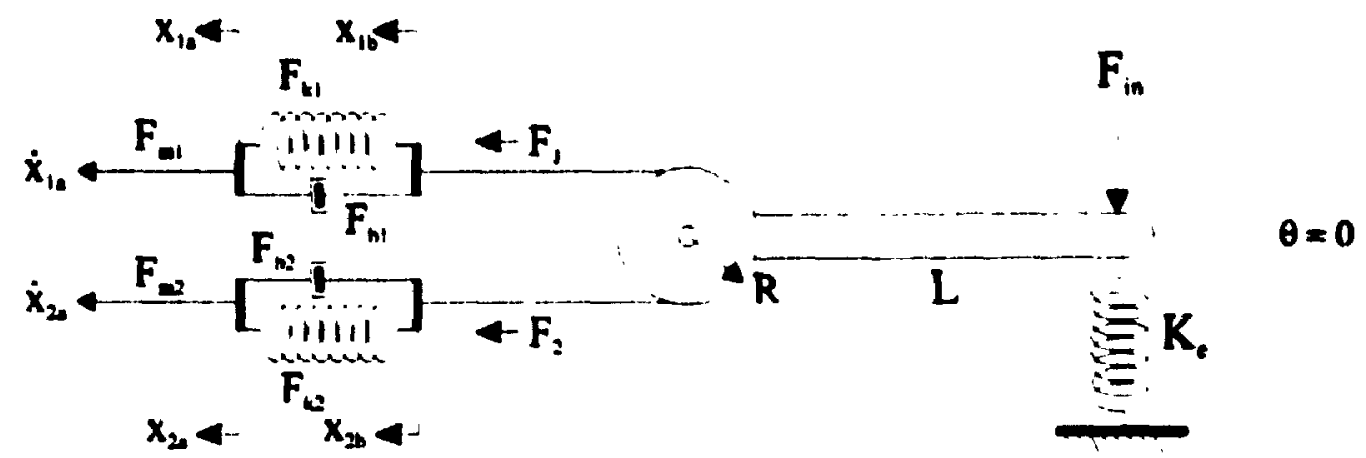

Figure 3.2. Basic actuator configurations

Table 3A: Parameter and variable definitions

\begin{tabular}{|c|c|}
\hline $\begin{array}{l}\text { parameter } \\
\text { or variable }\end{array}$ & definition \\
\hline$x_{10}, x_{2 a}$ & input position for branches $1 \& 2$ \\
\hline$x_{1 h}, x_{2 h}$ & position of joint end of component pairs \\
\hline$F_{m 1}, F_{m 2}$ & force at branch inputs \\
\hline$F_{k 1}, F_{k 2}$ & force in springs $1 \& 2$ \\
\hline$\overline{k_{1}, k_{2}}$ & stiffness of springs $1 \& 2$ \\
\hline$F_{h 1}, F_{h_{2}}$ & force in dampers $1 \& 2$ \\
\hline$b_{1}, b_{2}$ & damping coefficient of dampers $1 \& 2$ \\
\hline$\overline{F_{1}, F_{2}}$ & force of branches $1 \& 2$ on joint radius \\
\hline $\mathbf{R}$ & joint radius \\
\hline $\bar{L}$ & length of arm \\
\hline $\mathbf{F}_{\text {in }}$ & weight forces \\
\hline$\overline{K_{\mathbf{e}}}$ & stiffness of environment \\
\hline
\end{tabular}


A bond graph representation of the system is shown in Figure 3.3. The inputs of the system are the actuation velocities, the external force $F_{a b}$, and the velocity of the environment (assumed to be zero). The 0 -junctions on the left show the common force on either side of the spring-damper pairs, i.e., $F_{1}=F_{m 1}$ and $F_{2}=F_{m 2}$ (see Figure 3.2). The 1-junctions between spring and damper pairs shows the common velocity difference.

Forces in both branches are converted to torques through the joint radius, taking note of the positive torque direction. At the other end of the graph are the environmental inputs. The 0 -junction represents the common force on the environment and arm surface. This force is caused by the stretch across the environmental spring. The 1-junction shows the consistency of endpoint velocity, while the torques due to $F_{i n}$ and the environment spring add. The summed force is then converted to a joint torque through the length of the arm, once again noting the direction of positive torque. The remaining 1-junction represents the joint, where the torques due to all connections are summed and act on the inertia of the joint.

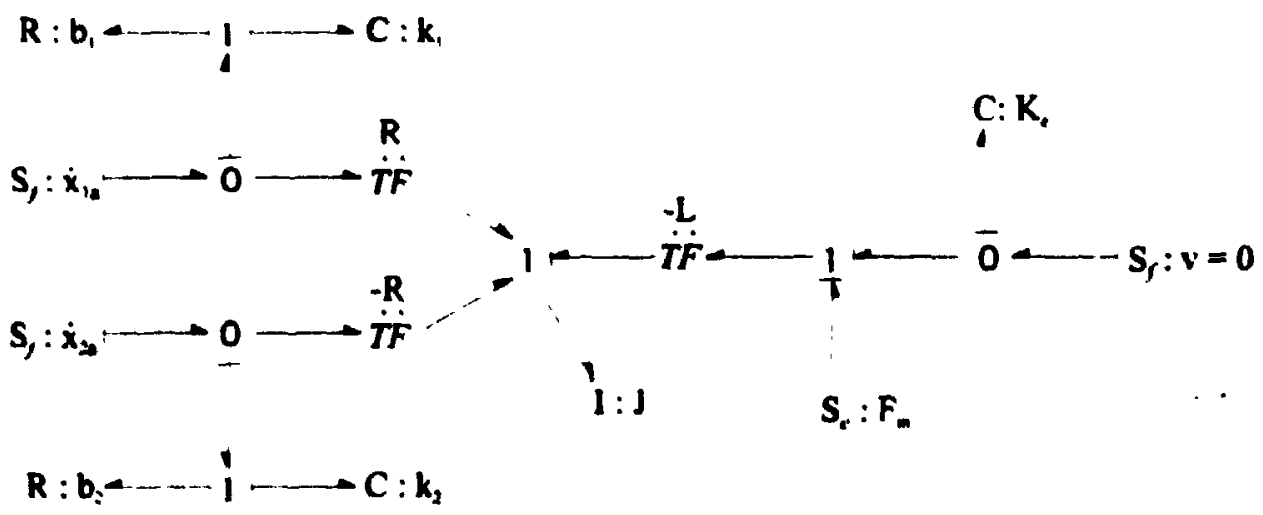

Figure 3.3: Bond graph of system 


\subsubsection{Sum and Difference Model}

For prosthetics, the configuration of Figure 3.2 could be implemented using activation of the bicep and tricep muscles to independently control branches 1 and 2 . However, it is more common in prosthetics research to use the sum of the activations as an indication of stiffness, and the difference to indicate position. The sum indicates the level of common activation, i.e., the amount that the muscles are pulled in the same direction. As in the model of Section 3.1, this corresponds to the level of joint stiffness. The difference of activation shows the level to which one muscle pulls more than the other. The equilibrium position thus moves towards the muscle with higher activation, as in the previsus section. Using these inputs, the bond graph is transformed as shown in Figure 3.4. Note that the summed inputs to the 0 -junctions on the left are the same as in Figure 3.3.

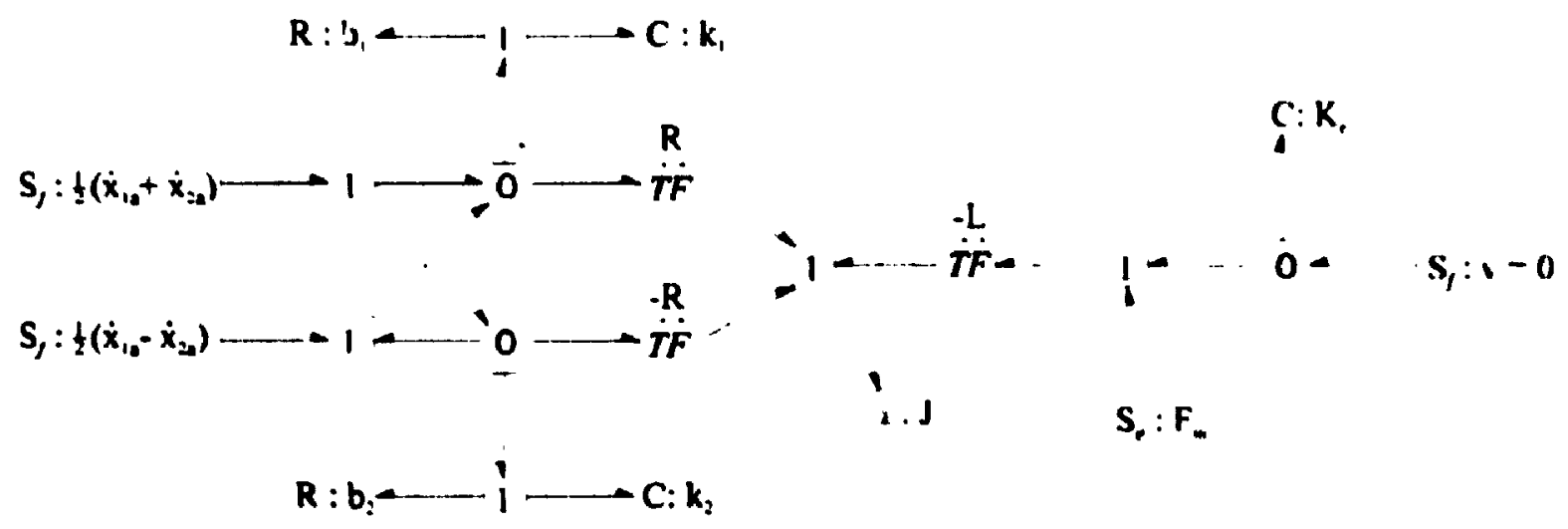

Figure 3.4: Change of inputs

The intention of this design is to create a compliant joint with variable stiffness. This is equivalent to having a torsion spring at the joint with the spring stiffness being 
controlled by extemal inputs. As previously mentioned, a property of bond graphs is the representation of equivalent systems using the same graphs. Therefore, it should be possible to manipulate the bond graph into an equivalent torsion spring form.

Figure 3.5(a) shows the movement of the position input across the graph. This is possible due to the property of superposition. In the previous representation (Figure 3.4), ihe inputs were summed before the spring and damper pairs. In this representation, the stiffness input is still introduced directly to the springs and dampers, but the position input comes between the components and the joint. A physical realization of this model is shown in Figure 3.5(b). The position input has moved to the joint so that the spring and damper pairs do not slide relative to each other for movements of the arm. It is necessary to have a non-backdrivable joint so that deflections of the arm are transmitted through the position input to the branch elemenis.

The next step in manipulation of the bond graph is the separation of the springdamper pairs (Figure 3.6). The physical realization of this model is the same as Figure 3.5(b), but the springs and dampers have been separated into four branches rather than two. The final manipulation combines the two springs and two dampers into stiffness and damping "fields", as shown in Figure 3.7(a). The stiffness field has a mathematical representation that is identical to the combination of the spring bonds between the two left-hand 1-junctions of Figure 3.6. Likewise, the damping field is mathematically represented by the combination of damper bonds. The physical realization of this bond graph is a variable stiffness torsion spring at the joint (Figure 3.7(b)). This confirms the original premise of equivalent systems. 


$$
S_{f}: t\left(\dot{x}_{10}-\dot{x}_{20}\right)
$$

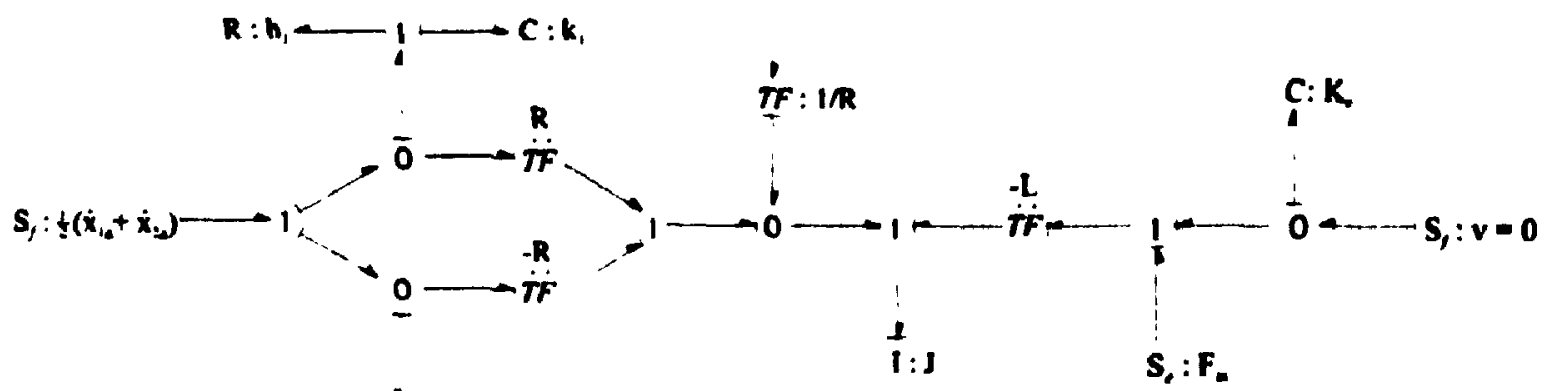

$$
R: b:-i-c: k:
$$

(a)

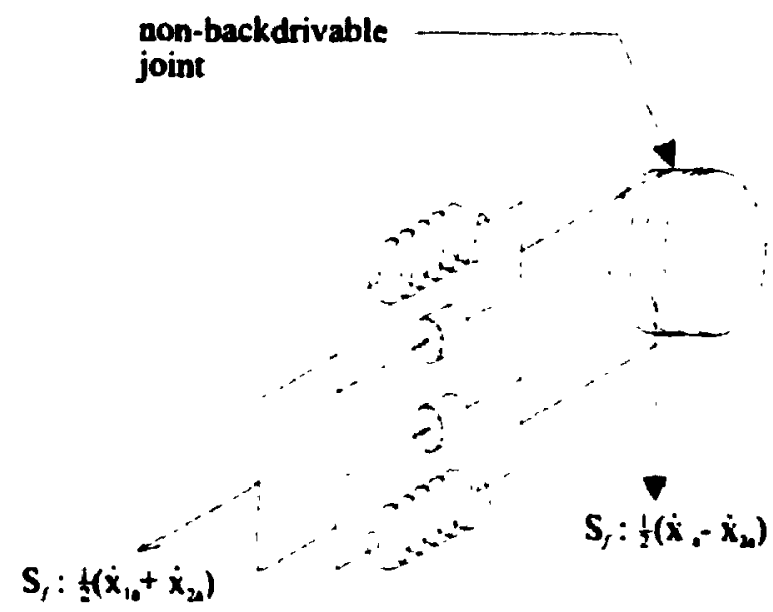

(b)

Figure 3.5: Manipulation of bond graph (a) movement of input, (b) physical representation.

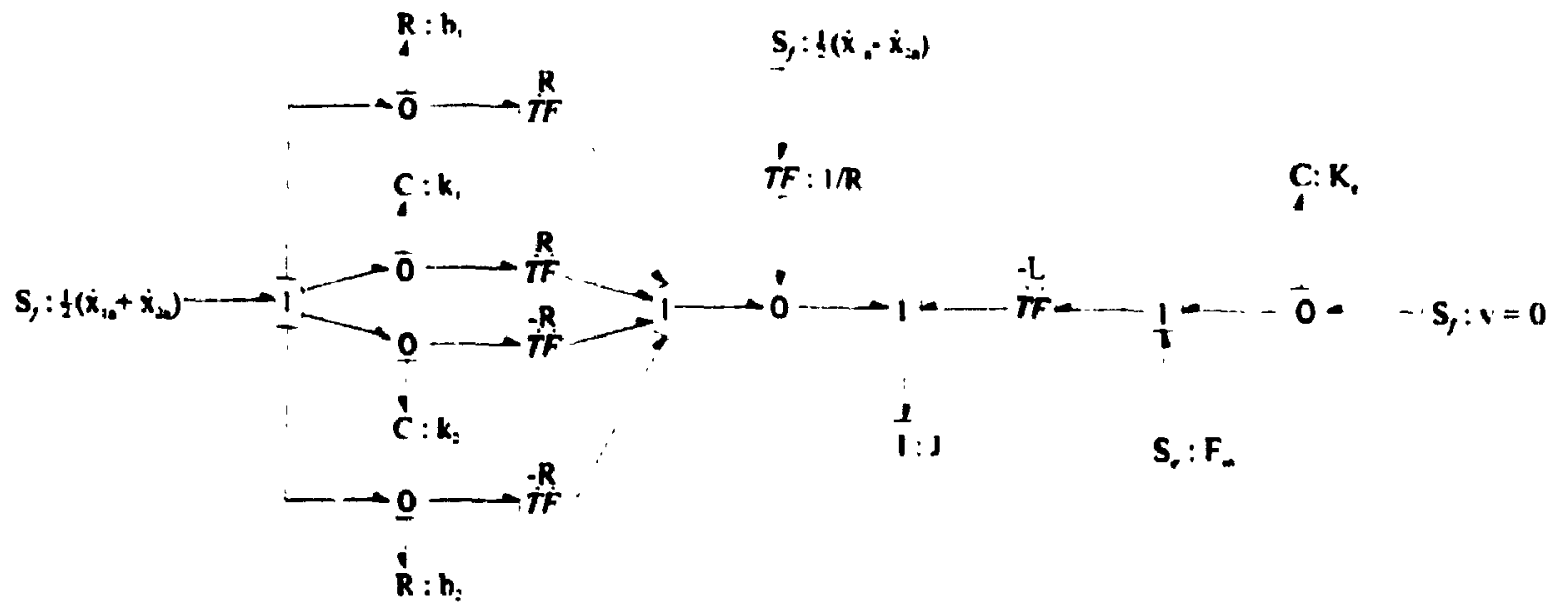

Figure 3.6: Separation of components

41 


$$
\begin{aligned}
& \text { C: } \mathbf{K} \text {, }
\end{aligned}
$$

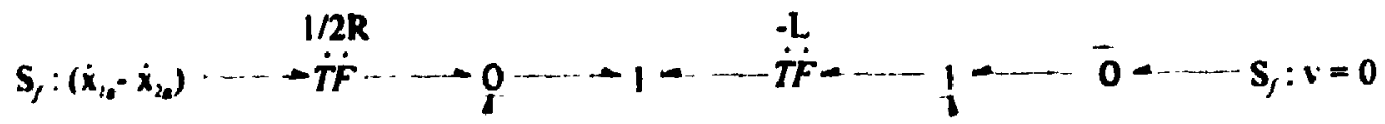

$$
\begin{aligned}
& \text { 1. } \quad 1: 3 \quad S_{*}: F_{m} \\
& \text { C R } \\
& \because \\
& S_{j}:\left(\dot{x}_{18}+\dot{x}_{21}\right) \cdots F: 1 / 2
\end{aligned}
$$

(a)

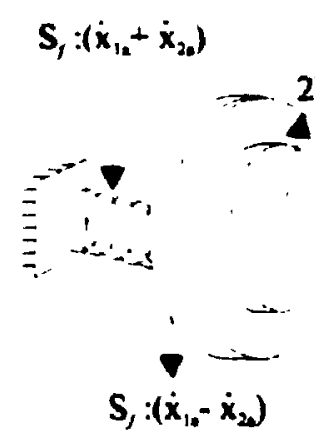

(b)

Figure 3.7. Combination into stiffness and damping fields. (a) bond graph representation. (b) physical realization as a variable stiffiness torsion spring. Note that the $1 / 2$ on each of the inputs has been moved to a transformer within the bond graph in (a), and is represented by a doubled radius in (b)

While the sum and difference configuration is ideal from the source point of view, it places limitations on the design and analysis of the actuation system. Once again it makes sense to apply direct control of the actuator based on muscle activation measurements. However, for most applications a control system is required to interpret the muscle signals as desired stiffness and position. This control system directs the 
motion of the actuators. Thus the controller would have to interpret the sum and difference as a desired stiffness and position. This would require that the stiffness be a mathematical function of the sum and the position be a function of the difference of the inputs (Figure 3.8(a)). Alternately they could both be a function of the sum and difference from which the stiffness and position could be determined (Figure 3.8(b)). The difficulty in this design is determining stiffness and position equations in terms of the sum and difference. Ideally, these equations would be decoupled as in Figure 3.8(a).

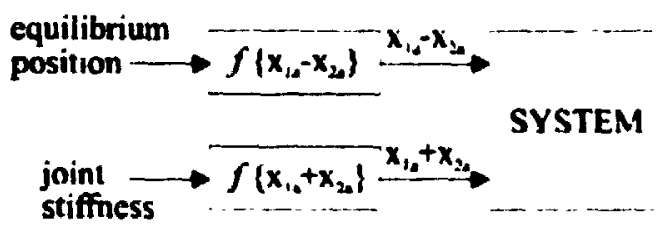

(a)

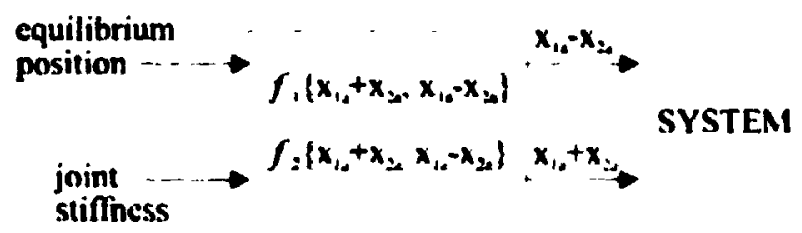

(b)

Figure 3.8. System inputs, (a) Sum and difference actuation, (b) coupled position and stiffness

\subsubsection{Stiffness and Position Model}

An alternate view of the actuation is to look at the control of $x_{12}$ and $x_{2 a}$ (Figure 3.2) as a combination of contributions from stiffness and position inputs. An example for $x_{1,}$ is shown in Figure 3.9. Table 3B defines the contributions of $x_{1 k}, x_{2 k}$, and $x_{\text {move }}$ in an ideal unloader situation. This type of control makes more sense for a robot manipulator since the position and stiffness are directly commanded, as in Figure 3.10(a), without the need for measurement of muscle activation. This technique is also viable for prosthetics since the controller must first convert the muscle activations to desired stiffness and position values that can be input directly into this system. The system at rest is shown in 
Figure 3.10(b) and the geometry of actuation is shown in Figure 3.10(c). A bond graph representation of the system using these inputs is shown in Figure 3.11.

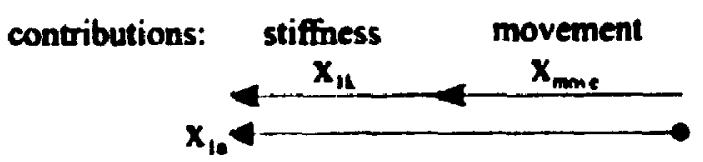

Figure 3.9. Stiffiness and position contributions to input $x_{1 .}$

Table 3B: Definition of stiffiness and position contributions (unloaded arm).

\begin{tabular}{|c|c|c|}
\hline & $\begin{array}{c}\text { change in } \\
\text { stored energy }\end{array}$ & $\begin{array}{c}\text { change in arm } \\
\text { position }\end{array}$ \\
\hline$x_{1 k}, x_{2 k}$ & yes & no \\
\hline$x_{\text {mmie }}$ & no & yes \\
\hline
\end{tabular}

The branch position inputs are defined as $x_{1 a}$ and $x_{2 a}$ (Figure 3.2). These represent the position that the actuators control to vary position and stiffness. The equilibrium position, $\theta_{o q}$, represents the commanded position of the arm. It is the position where the arm would come to rest if all external loading was removed. This position is set by the $x_{\text {mor }}$ contribution of the inputs (Figure 3.9). Note by geometry from Figure 3.10(c),

$$
x_{\text {mose }}=R \theta_{\infty}
$$

The stiffness contributions of the inputs (Figure 3.9) are defined as $x_{11}$ and $x_{2 k}$. The inputs are therefore given by (Figure 3.10(c)),

$$
\begin{aligned}
& x_{10}=x_{1 k}+x_{\text {move }} \\
& x_{20}=x_{2 k}-x_{\text {move }}
\end{aligned}
$$


The positions of the joint side of the branch components are denoted $x_{1 b}$ and $x_{2 b}$. By geometry from Figure $3.10(\mathrm{c})$, the stretches of the springs are

$$
\begin{aligned}
& \delta_{1}=x_{14}-x_{1 b} \\
& \delta_{2}=x_{21}-x_{21}
\end{aligned}
$$

Both $x_{1 b}$ and $x_{2 b}$ can be changed by an input position change, $x_{\text {mone }}$ or by deflection of the joint when it is loaded. The contribution of joint deflection tc $x_{1 b}$ and $x_{2 b}$ is denoted as $x_{\text {lood. }}$. From the geometry in Figure $3.10(\mathrm{c})$ it can be seen that

$$
\begin{aligned}
& x_{1 b}=x_{\text {more }}+x_{\text {lood }} \\
& x_{2 b}=-\left(x_{\text {move }}+x_{\text {boed }}\right)
\end{aligned}
$$

Deflection of the joint is the difference between actual position and commanded position of the arm (Figure 3.10(c)). The deflection is therefore defined as

$$
\Delta=\theta-\theta_{\text {aq }}
$$

By Figure 3.10(c), the relationship between joint deflection and $x_{\text {ked }}$ is

$$
x_{\text {ked }}=R\left(\theta-\theta_{\text {eq }}\right)=R \Delta
$$

Substituting equations (3-1), (3-2a), (3-2b), (3-4a), (3-4b), and (3-6) into equations (3-3a) and $(3-3 b)$,

$$
\begin{aligned}
& \delta_{1}=x_{1 k}-R \Delta \\
& \delta_{2}=x_{2 k}+R \Delta
\end{aligned}
$$



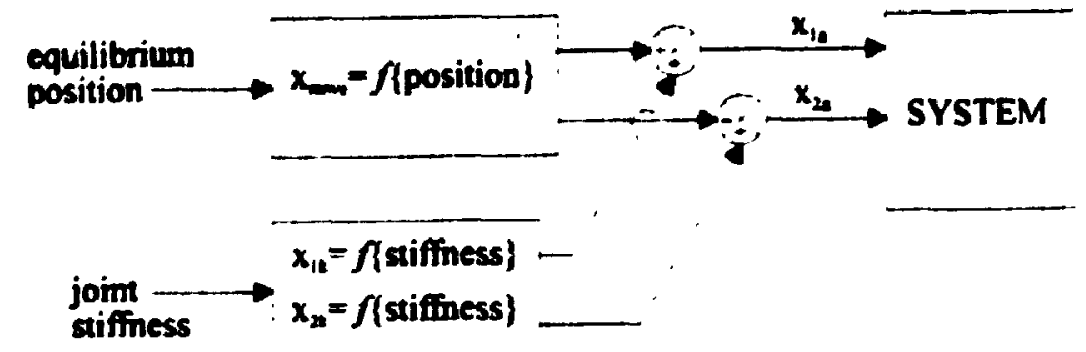

(a)

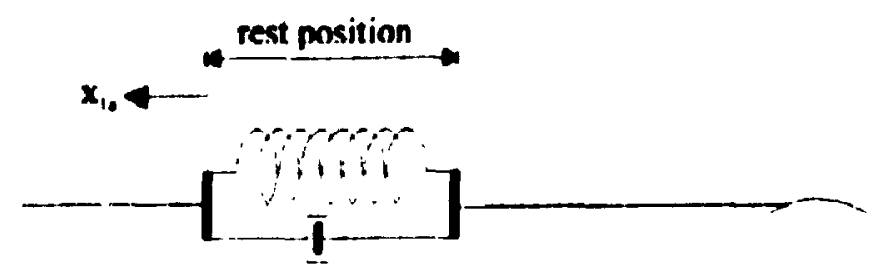

$\theta=0$

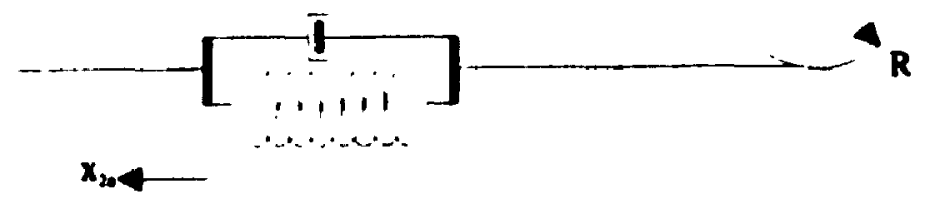

(b)

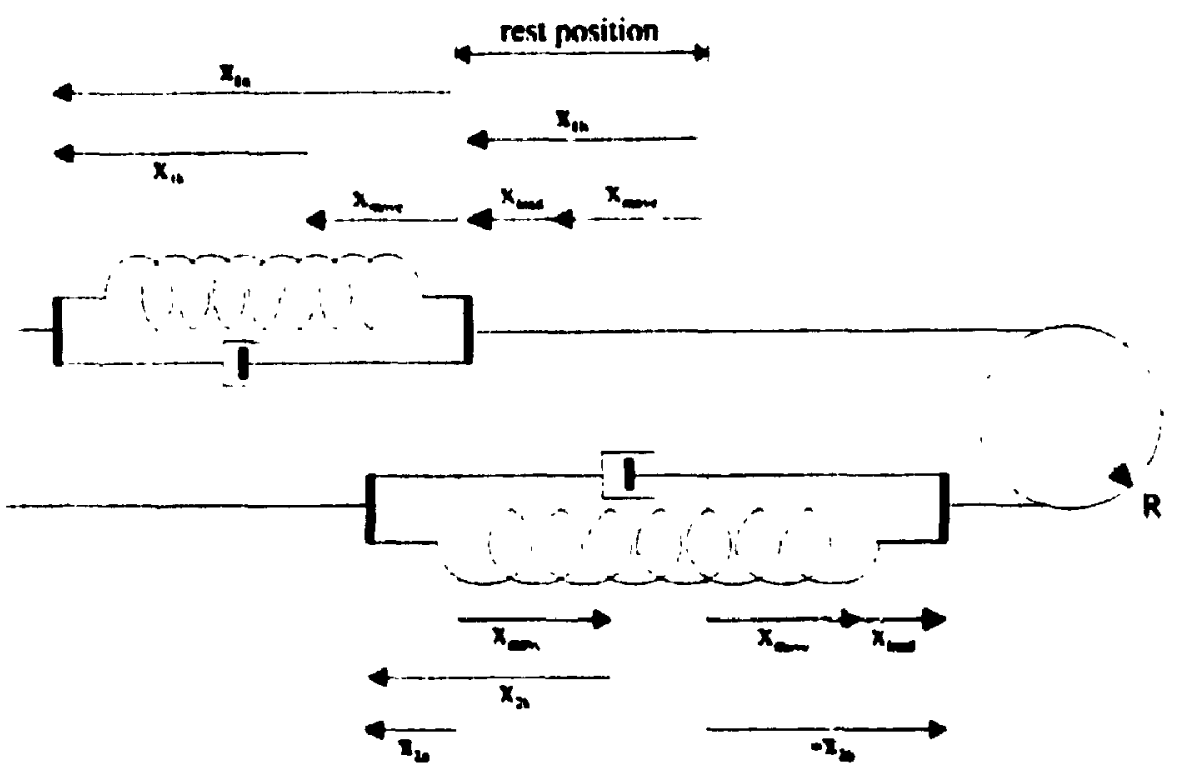

(c)

Figure 3.10 Inputs as sum of stiffness and position contributions, (a) block diagram, (b) system at rest, (c) geometry of actuation. 


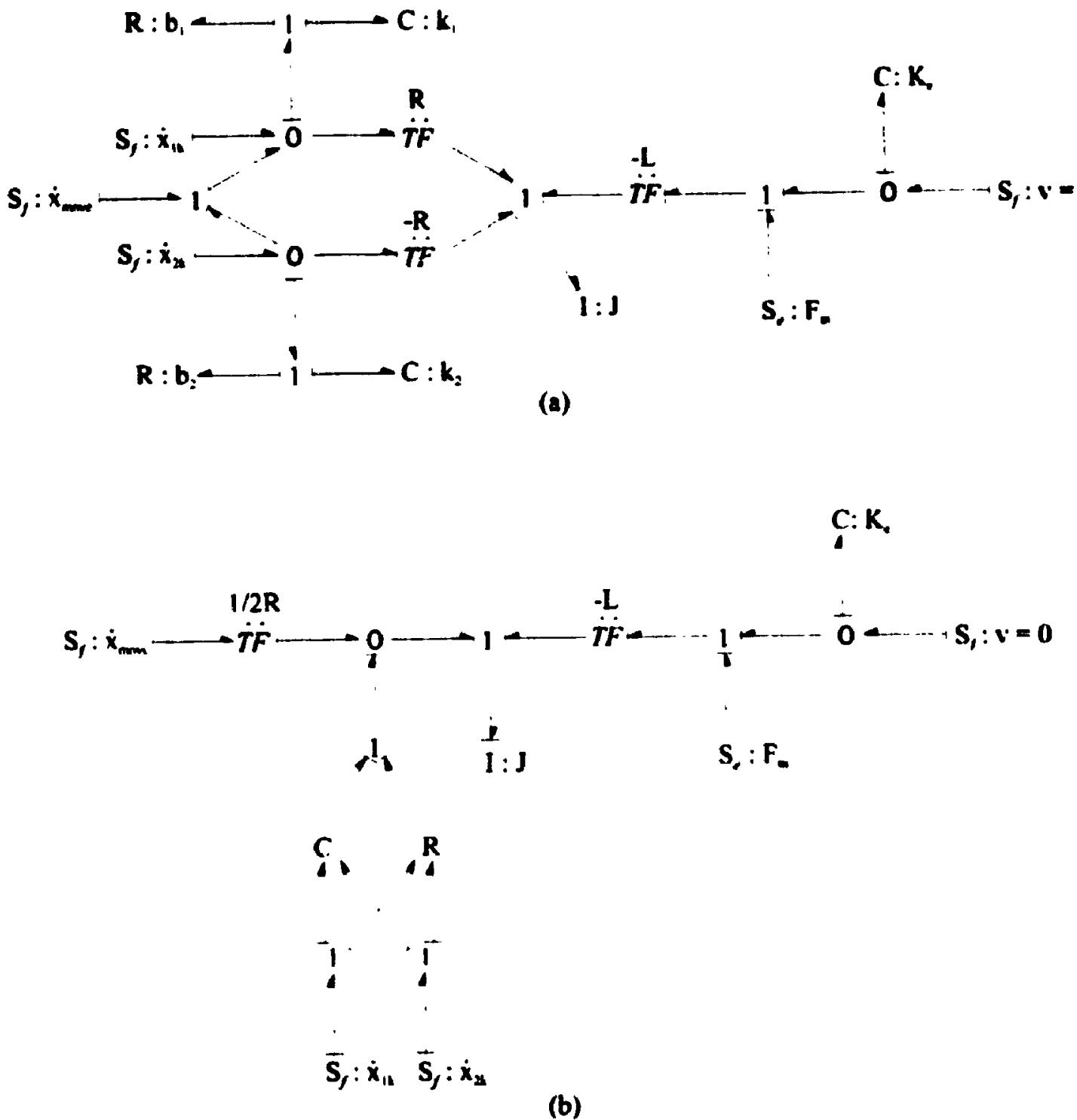

Figure 3.11: Bond graph representation using stifiness and position inputs, (a) design model, (b) manipulated bond graph in form of variable torsion spring. 


\subsubsection{Mechanics}

The effect of the springs and dampers on the joint can be shown through their torque contribution. In static equilibrium there is no force caused by the dampers and there is no inertial torque. The sum of torques at the joint in equilibrium (Figure 3.12) is

$$
\begin{aligned}
T_{\infty x} & +R \cdot F_{1}-R \cdot F_{2}=0 \\
\text { or } \quad T_{\infty x} & =R \cdot\left(F_{2}-F_{1}\right)
\end{aligned}
$$

where $T_{e x i}$ is the external torque.

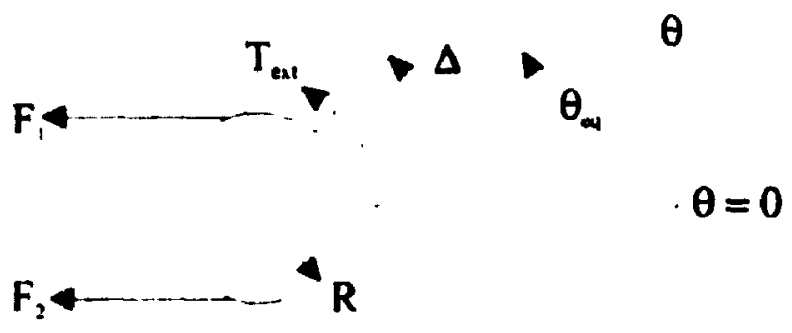

Figure 3 12: Sum of joint torques in equilibrium.

It will be shown that for the joint stiffness to vary, the springs must be non-linear. This means that the stiffness is not constant and force is not directly proportional to spring deflection. The spring forces can therefore be defined, in general, as

$$
\begin{aligned}
& F_{k 1}=\phi_{1}\left\{\delta_{1}\right\} \\
& F_{k 2}=\phi_{2}\left\{\delta_{2}\right\}
\end{aligned}
$$

where $\phi_{1}$ and $\phi_{2}$ are the unknown force functions. Equation (3-8) then becomes

$$
T_{e x}=R \cdot\left(\phi_{2}\left\{\delta_{2}\right\}-\phi_{1}\left(\delta_{1}\right\}\right)
$$


The stiffness of an element is defined as the change in force (or torque) due to a change in deflection of the element. Assuming a constant radius, the effective joint stiffness is defined as

$$
K^{*}=\frac{d T_{e x}}{d \Delta}=R \cdot\left(\frac{\partial \phi_{2}}{\partial \Delta}-\frac{\partial \phi_{1}}{\partial \Delta}\right)
$$

By the chain rule, equation (3-11) becomes

$$
K^{*}=R \cdot\left(\frac{\partial \phi_{2}}{\partial \delta_{2}} \cdot \frac{d \delta_{2}}{d \Delta}-\frac{\partial \phi_{1}}{\partial \delta_{1}} \cdot \frac{d \delta_{1}}{d \Delta}\right)
$$

Differentiating equations (3-7a) and (3-7b) with respect to $\Delta$,

$$
\begin{aligned}
& \frac{d \delta_{1}}{d \Delta}=-R \\
& \frac{d \delta_{2}}{d \Delta}=R
\end{aligned}
$$

The derivative of force with deflection is defined as the stiffness. Assuming the general case of non-linear springs, the stiffnesses are not constant and are a function of deflection. Thus the spring stiffnesses are defined as

$$
\begin{aligned}
& k_{1}=\psi_{1}\left\{\delta_{1}\right\}=\frac{\partial \phi_{1}}{\partial \delta_{1}} \\
& k_{2}=\psi_{2}\left\{\delta_{2}\right\}=\frac{\partial \phi_{2}}{\partial \delta_{2}}
\end{aligned}
$$

where $\psi_{1}$ and $\psi_{2}$ are the unknown stiffness functions. Substituting equations (3-13a) through (3-14b) into equation (3-12),

$$
\mathrm{K}^{*}=\mathrm{R}^{2} \cdot\left(\psi_{1}\left\{\delta_{1}\right\}+\psi_{2}\left\{\delta_{2}\right\}\right)
$$

If the springs were linear, i.e., constant stiffnesses, the resulting joint stiffness would also be constant. The joint stiffness in equation (3-15) is only variable if the individual stiffnesses, or joint radius, are variable, confirming the above assumption of 
non-linear springs. This allows the joint to act as a regular rotational spring with the stiffness value defined by the deflections of the individual springs. Figure 3.11 (b) shows this relationship in bond graph form. This confirms that $\psi$ must be a non-constant function of $\delta$ and thus $\phi$ is non-linear as previously stated. 


\section{Chapter 4}

\section{System Performance}

This chapter presents two aspects of the system's performance: design based on desired performance characteristics, and the abilities and limitations that result from design. The former of these aspects deals with matching design criteria. It is determined what design specifications, particularly in the form of spring functions, are necessary to meet these criteria. An analysis of the resulting limitations and performance characteristics, such as power and energy requirements, indicates the abilities that can be achieved by this design. In addition, this analysis suggests directions for improvements and future research.

\subsection{Selection of Spring Functions}

There have been several suggested implementations of passive impedance control using mechanical components [Goswami et al., 1990; Cutkosky \& Wright, 1986; Immega, 1987; Mills, 1990; Yi \& Freeman, 1992; Mittal et al., 1993]. The problen. with 
most of these schemes is that the stiffness is coupled to position, i.e., it changes with deflection from the set position. This means that one cannot know the actual stiffiness value unless there is position feedback. In dynamic situations where the deflection is rapidly changing, the same feedback problems arise as in active impedance control (see Section 2.1.2). Ideally the stiffness of the elbow should be independent of endpoint deflection. The programmable passive impedance components developed by LaurinKovitz et al. [1991] avoid this problem by using quadratic springs that, by their nature, decouple stiffness from position and deflection. This section examines the relationship of spring function to decoupling of joint stiffness and position.

\subsubsection{Stiffness Function}

It is desired that the joint stiffness is not a function of deflection, or $K^{\bullet} \neq f\{\Delta\}$. Thus,

$$
\frac{d K^{*}}{d \Delta}=0
$$

Differentiating equation $(\hat{\jmath}-\hat{\imath} \leq)$,

$$
\frac{d K^{\bullet}}{d \Delta}=R^{2} \cdot\left(\frac{d \psi_{1}}{d \Delta}+\frac{d \Psi_{2}}{d \Delta}\right)=0
$$

Canceling the radius and using the chain rule,

$$
\frac{\partial \psi_{1}}{\partial \delta_{1}} \cdot \frac{d \delta_{1}}{d \Delta}+\frac{\partial \psi_{2}}{\partial \delta_{2}} \cdot \frac{d \delta_{2}}{d \Delta}=0
$$

Substituting equations (3-13a) and (3-13b) into equation (4-1),

$$
\frac{\partial \psi_{1}}{\partial \delta_{1}}(-R)+\frac{\partial \psi_{2}}{\partial \delta_{2}}(R)=0
$$

Simplifying, 


$$
\frac{\partial \psi_{1}}{\partial \delta_{1}}=\frac{\partial \psi_{2}}{\partial \delta_{2}}
$$

A graphical interpretation of equation (4-2) is that the slopes of the stiffness curves must be identical as in Figure 4.1. By equations (3-7a) and (3-7b), the values of $\delta_{1}$ and $\delta_{2}$ can change by two means; either the stiffness changes $\left(x_{1 k}, x_{2 k}\right)$ or the deflecticn changes ( $\Delta$ ). Equation (4-2) must hold for both cases. This means that whether there is a change in stiffness or a change in deflection the slopes of the new operating points must still remain equal. Figure 4.1 (a) shows a stretch of spring 1 that could be due to either a change of stiffness $\left(x_{1 k 0}\right.$ to $\left.x_{1 k 1}\right)$ or a deflection $\left(\Delta_{0}\right.$ to $\left.\Delta_{2}\right)$. (Note that the subscript "0" refers to the original position, "1" refers to a change in stiffness, and "2" refers to a change in deflection.) Figure 4.1(b) shows the resulting stretch in both cases for spring 2. The slope must be the same whether there is stiffness change or deflection. A stiffness change pulls both springs in the same direction whereas a joint deflection causes deflection of the springs in opposite directions. This means that the slope must be identical on either side of the initial position $\left(x_{1 k 0}+R \Delta_{0}, x_{2 k 0}-R \Delta_{0}\right)$. Since the initial position is arbitrary, the only curve that can fulfill this requirement is one with a constant slope, i.e., a straight line (Figure 4.2(a), end (b)). Thus the stiffness curve must be linear (i.e. quadratic force curve). Below is a mathematical proof of these arguments.

The above arguments say that a change in slope must be the same for both springs, whether due to a stiffness change or endpoint deflection. A change in deflection is represented by $\Delta$. A change in stiffness is represented by a change in $x_{1 k}$ and $x_{2 k}$. However, $x_{11}$ and $x_{2 k}$ must be related such that as one changes, the other changes an amount that will keep the static forces in equilibrium so that there is no motion or output force change, i.e., $x_{2 k}=f\left(x_{1 k}\right\}$. Thus a change in stiffness can be represented by a change in either variable. 
$\psi_{4}$

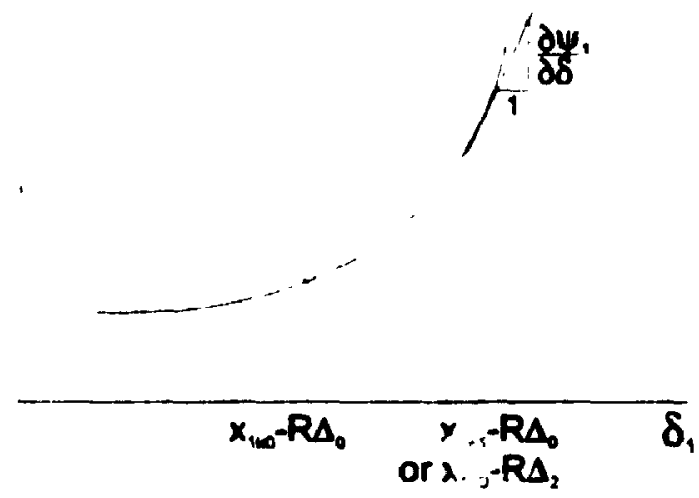

(a) $\psi_{2}$

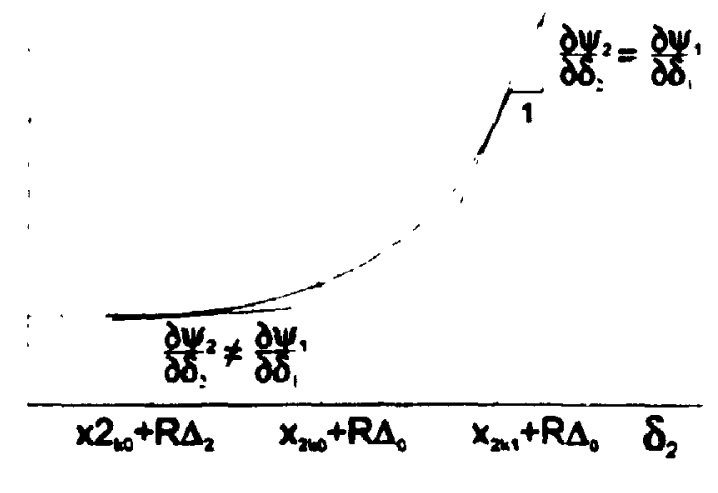

(b)

Figure 4 I Abitrary stiffness functions. (a) stretch r, ving i due to change in stiffness,

(b) coincident stretch of spring 2 for both cases.

$\Psi_{1}$

$\Psi_{2}$

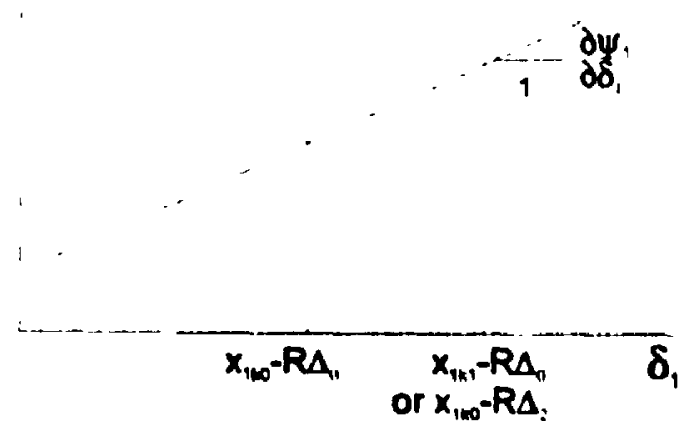

(a)

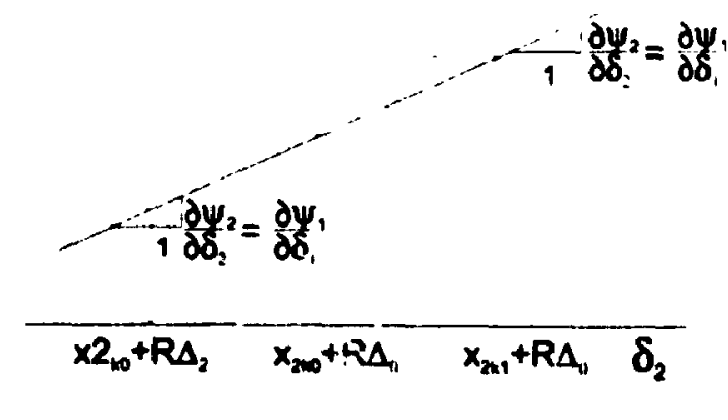

(b)

Figure 4.2: Linear stiffness functions (á) spring l, (b) corresponding spring 2. 
Taking the derivative of equation (4-2) with respect to a deflection change,

$$
\frac{d}{d \Delta}\left(\frac{\partial \psi_{1}}{\partial \delta_{1}}\right)=\frac{d}{d \Delta}\left(\frac{\partial \psi_{2}}{\partial \delta_{2}}\right)
$$

Using the chain rule,

$$
\frac{\partial}{\partial \delta_{1}}\left(\frac{\partial \psi_{1}}{\partial \delta_{1}}\right) \cdot \frac{d \delta_{1}}{d \Delta}=\frac{\partial}{\partial \delta_{2}}\left(\frac{\partial \psi_{2}}{\partial \delta_{2}}\right) \cdot \frac{d \delta_{2}}{d \Delta}
$$

Substituting equations (3-13a) and (3-13b) and simplifying,

$$
\frac{d^{2} \Psi_{1}}{d \delta_{1}^{2}}=-\frac{d^{2} \Psi_{2}}{d \delta_{2}^{2}}
$$

Equation (4-3) suggests that the curvature of the two stiffness functions must be opposite. This means that the curves in Figure 4.1 would not be sufficient, as suggested by the figure. Taking the derivative of equation (4-2) with respect to a stiffness change,

$$
\frac{d}{d x_{1 h}} \cdot\left(\frac{\partial \psi_{1}}{\partial \delta_{1}}\right)=\frac{d}{d x_{1 \mathrm{~h}}} \cdot\left(\frac{\partial \psi_{2}}{\partial \delta_{2}}\right)
$$

By the chain rule,

$$
\frac{\partial}{\partial \delta_{1}}\left(\frac{\partial \psi_{1}}{\partial \delta_{1}}\right) \cdot \frac{d \delta_{1}}{d x_{1 k}}=\frac{\partial}{\partial \delta_{2}}\left(\frac{\partial \psi_{2}}{\partial \delta_{2}}\right) \cdot \frac{d \delta_{2}}{d x_{2 k}} \cdot \frac{\partial x_{2 k}}{\partial x_{1 k}}
$$

Differentiating equations (3-7a) and (3-7b),

$$
\begin{aligned}
& \frac{d \delta_{1}}{d x_{1 k}}=1 \\
& \frac{d \delta_{2}}{d x_{2 k}}=1
\end{aligned}
$$

Substituting equations (4-5a) and (4-5b) into equation (4-4),

$$
\frac{\partial^{2} \Psi_{1}}{\partial \delta_{1}^{2}}=\frac{\partial^{2} \Psi_{2}}{\partial \delta_{2}^{2}} \cdot \frac{\partial x_{2 k}}{\partial x_{1 k}}
$$


Equation (4-6) suggests that the curvatures of the stiffness functions are related by $x_{1 h}$ and $x_{21}$. Substituting equation (4-6) into equation (4-3) and simplifying.

$$
\frac{\partial^{2} \Psi_{2}}{\partial \delta_{2}^{2}} \cdot\left(1+\frac{\partial x_{2 k}}{\partial x_{11}}\right)=0
$$

There are two solutions to this equation. The first case assumes that

$$
1+\frac{\partial x_{2 k}}{\partial x_{1 k}}=0
$$

To check if this case is possible it is necessary to find the relationship between $x_{11}$ and $x_{21}$. By equation (3-11),

$$
T_{e v}=\int_{\lambda_{0}}^{1_{1}} K^{\cdot} \cdot d \Delta=K^{*}\left(\Delta_{1}-\Delta_{0}\right)+T_{0}
$$

since it is desired that $\mathrm{K}^{*}$ is not a function of $\Delta$. Assuming no initial deflection or torque, this reduces to

$$
T_{e v}=K^{*} \Delta
$$

Comparing equation (4-9) with equation (3-10), substituting equatic.1 (3-15), and simplifying.

$$
\phi_{2}\left\{\delta_{2}\right\}-\phi_{1}\left\{\delta_{1}\right\}=\operatorname{R\Delta }\left(\psi_{1}\left\{\delta_{1}\right\}+\psi_{2}\left\{\delta_{2}\right\}\right)
$$

This force equilibrium equation must hold true for a change in stiffness without movement. Taking the derivative of equation (4-10) with respect to a stiffness change $\left(x_{1 k}\right)$ and using the chain rule.

$$
\frac{\partial \phi_{2}}{\partial \delta_{2}} \cdot \frac{d \delta_{2}}{d x_{2 k}} \cdot \frac{\partial x_{2 k}}{\partial x_{1 k}}-\frac{\partial \phi_{1}}{\partial \delta_{1}} \cdot \frac{d \delta_{1}}{d x_{1 k}}=R \Delta\left(\frac{\partial \psi_{1}}{\partial \delta_{1}} \cdot \frac{d \delta_{1}}{d x_{1 k}}+\frac{\partial \psi_{2}}{\partial \delta_{2}} \cdot \frac{d \delta_{2}}{d x_{2 k}} \cdot \frac{\partial x_{2 k}}{\partial x_{1 k}}\right)
$$

Substituting in equations (3-14a), (3-14b), (4-5a), and (4-5b),

$$
\psi_{2}\left\{\delta_{2}\right\} \cdot \frac{\partial x_{2 k}}{\partial x_{1 k}}-\psi_{1}\left\{\delta_{1}\right\}=R \Delta\left(\frac{\partial \psi_{1}}{\partial \delta_{1}}+\frac{\partial \psi_{2}}{\partial \delta_{2}} \cdot \frac{\partial x_{2 k}}{\partial x_{1 h}}\right)
$$

Substituting equation (4-2) into equation (4-11), 


$$
\psi_{2}\left\{\delta_{2}\right\} \cdot \frac{\partial x_{2 k}}{\partial x_{1 k}}-\psi_{1}\left\{\delta_{1}\right\}=R \Delta \cdot \frac{\partial \psi_{1}}{\partial \delta_{1}}\left(1+\frac{\partial x_{2 L}}{\partial x_{1 \mathrm{t}}}\right)
$$

Assuming equation (4-8) is true, the right-hand side of equation (4-12) becomes equal to zero, so

$$
\frac{\partial \mathrm{x}_{2 \mathrm{k}}}{\partial \mathrm{x}_{1 \mathrm{k}}}=\frac{\Psi_{1}\left\{\delta_{1}\right\}}{\Psi_{2}\left\{\delta_{2}\right\}}=-1
$$

Substituting equation (4-13) into (3-15),

$$
K^{*}=R^{2} \cdot\left\{\psi_{1}\left\{\delta_{1}\right\}-\psi_{1}\left\{\delta_{1}\right\}\right)=0
$$

which means that when equation (4-8) is true, $K^{\bullet}=0$. This is a trivial solution since it is desired to have a variable stiffness joint.

The second solution for equati', $n$ (4-8) assumes

$$
\frac{\partial^{2} \Psi_{2}}{\partial \delta_{2}{ }^{2}}=0
$$

Comparing equations (4-15) and (4-3),

$$
\frac{\partial^{2} \Psi_{1}}{\partial \delta_{1}{ }^{2}}=-\frac{\partial^{2} \Psi_{2}}{\partial \delta_{2}^{2}}=0
$$

Therefore equation (4-2) becomes

$$
\frac{\partial \psi_{1}}{\partial \delta_{1}}=\frac{\partial \psi_{2}}{\partial \delta_{2}}=\mathrm{A}
$$

where $A$ is a constant of integration. A second integration produces

$$
\begin{aligned}
& \psi_{1}\left\{\delta_{1}\right\}=A \delta_{1}+B \\
& \psi_{2}\left\{\delta_{2}\right\}=A \delta_{2}+C
\end{aligned}
$$

where $B$ and $C$ are constants of integration. Thus springs with stiffnesses that vary linearly with stretch provide the only solution for decoupling joint stiffness from deflection. This confirms the graphical proof presented earlier. (Note: an altemate proof is provided in Appendix A.) 
For each spring, the stiffness at zero stretch is defined as minimum stiffness. Substitution of zero stretch into equations (4-17a) and (4-17b) result in

$$
\begin{aligned}
& \psi_{1}\{0\}=k_{1 \min }=B \\
& \psi_{2}\{0\}=k_{2 \min }=C
\end{aligned}
$$

Defining $\delta_{1 \text { pox }}$ and $\delta_{2 p o s}$ as the maximum positive stretch of springs 1 and 2 , respectively, the stiffness at maximum stretch is defined as the maximum stiffness. Combining equations (4-17a), (4-17b), (4-18a), and (4-18b) and substituting maximum stretch,

$$
\begin{aligned}
& \psi_{1}\left\{\delta_{1 \text { pos }}\right\}=A \delta_{1 \text { pus }}+k_{1 \text { min }}=k_{1 \text { mav }} \\
& \psi_{2}\left\{\delta_{2 \text { pos }}\right\}=A \delta_{2 \text { pas }}+k_{2 \min }=k_{2 \mathrm{max}}
\end{aligned}
$$

Rearranging equations (4-19a) and (4-19b),

$$
A=\frac{k_{1 \max }-k_{1 \min }}{\delta_{1 \text { mos }}}=\frac{k_{2 \max }-k_{2 \min }}{\delta_{2 \text { mas }}}
$$

Substituting $A, B$, and $C$ into equations (4-17a) and (4-17b),

$$
\begin{aligned}
& \psi_{1}\left\{\delta_{1}\right\}=\frac{k_{1 \max }-k_{1 \min }}{\delta_{1 \mathrm{max}}} \cdot \delta_{1}+k_{1 \min } \\
& \psi_{2}\left\{\delta_{2}\right\}=\frac{k_{2 \operatorname{ma}}-k_{2 \min }}{\delta_{2 \mathrm{mas}}} \cdot \delta_{2}+k_{2 \mathrm{~min}}
\end{aligned}
$$

Substitution of equations (4-21a) and (4-21b) into equation (3-15) gives the joint stiffness as

$$
K^{*}=R^{2}\left(\frac{k_{1 \max }-k_{1 \min }}{\delta_{1 \text { pos }}} \cdot \delta_{1}+k_{1 \min }+\frac{k_{2 \max }-k_{2 \min }}{\delta_{2 p \operatorname{mos}}} \cdot \delta_{2}+k_{2 \min }\right)
$$

However, the $\delta_{1}$ and $\delta_{2}$ terms can be combined, noting that their coefficients are equivalent by equation (4-20). This reduces equation (4-22) to

$$
\mathrm{K}^{\bullet}=\mathrm{R}^{2}\left(\mathrm{~A}\left(\delta_{1}+\delta_{2}\right)+\mathrm{k}_{1 \mathrm{~min}}+\mathrm{k}_{2 \mathrm{mn}}\right)
$$


Since the design calls for a range of joint stiffnesses rather than the individual springs, it is better to put the constants in terms of joint stiffness limits. With no spring deflection $\left(\delta_{1}=\delta_{2}=0\right)$, the joint is defined as having minimum stiffness. Thus by equation (4-23).

$$
K_{\min }^{*}=R^{2}\left(k_{1 \min }+k_{2 \min }\right)
$$

When both springs are stretched to their maximum $\left(\delta_{1}=\delta_{1 p a n}, \delta_{2}=\delta_{2 p m}\right)$, the joint is defined as having maximum stiffness. Equation (4-24) becomes

$$
K_{\max }^{*}=R^{2}\left(A\left(\delta_{1 p o s}+\delta_{2 p o s}\right)+k_{1 \operatorname{man}}+k_{2 \operatorname{man}}\right)
$$

Substituting equation (4-24) into equation (4-25) and rearranging,

$$
A=\frac{K_{\text {max }}^{\cdot}-K_{\text {min }}^{\bullet}}{R^{2}\left(\delta_{1 \text { pos }}+\delta_{2 \operatorname{pos}}\right)}
$$

Substitution of equations (4-26) and (4-24) into equation (4-23) gives the joint stiffness as

$$
K^{\cdot}=\frac{K_{\operatorname{man}}^{\cdot}-K_{\min }^{\cdot}}{\delta_{1 p o s}+\delta_{2 p o s}}\left(\delta_{1}+\delta_{2}\right)+K_{\min }^{\cdot}
$$

It is interesting to note that combining equations (3-7a) and (3-7b) shows that

$$
\delta_{1}+\delta_{2}=x_{1 k}+x_{2 k}
$$

Substituting equation (4-28) into equation (4-27),

$$
K^{\bullet}=\frac{K_{\max }^{\cdot}-K_{\min }^{\cdot}}{\delta_{1 p o s}+\delta_{2 p o s}}\left(x_{1 k}+x_{2 k}\right)+K_{\min }^{\cdot}
$$

so the joint stiffness is not a function of $\Delta$. This means that equation (4-15) provides a usable solution for decoupling stiffness and position. In addition, it proves that the only solution for such decoupling in the presented configuration is to use springs with linearly increasing stiffnesses. It is also interesting to note that combining equations (3-2a), (3$2 b)$, and (4-28) shows that

$$
x_{1 \mathrm{a}}+x_{2 \mathrm{a}}=x_{1 \mathrm{k}}+x_{2 \mathrm{k}}=\delta_{1}+\delta_{2}
$$


This means that stiffness can also be controlled by the sum of the inputs as suggested in Section 3.1.1.

\subsubsection{Position Function}

By equation (3-1),

$$
\theta_{\infty \mathrm{q}}=\frac{\mathrm{X}_{\text {more }}}{\mathrm{R}}
$$

Thus position is a function of $x_{\text {more }}$ Equations (4-29) and (4-31) show that separating the inputs into stiffness and position contributions, as suggested in Section 3.1.2, can successfully be implemented.

It has already been shown by equations (4-29) and (4-30) that stiffness can be controlled by the sum of the inputs. It is then interesting to check that position can be controlled by using the difference of the inputs as a command for the system suggested in Section 3.1.1. Subtraction of equation (3-2b) from (3-2a) gives the difference of inputs as

$$
x_{10}-x_{2 a}=x_{1 \mathrm{~L}}-x_{2 \mathrm{~h}}+2 \mathrm{x}_{\text {mole }}
$$

Rearranging equation (4-32) to solve for $x_{\text {more }}$ and substituting into equation (4-31),

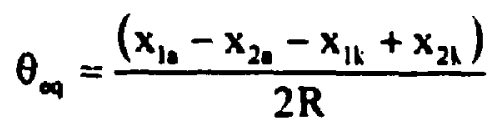

This shows that position can be controlled by the difference of inputs if $x_{1 k}=x_{2 k}$ for all joint stiffnesses. Changing joint stiffness without motion involves changing the stretch of both springs. This must not affect the equilibrium of forces created by the springs at the joint or else equilibrium position movement will occur. Thus for a given change in $x_{1 k}$, the corresponding change in $x_{2 k}$ is that which produces no net torque at the joint when unloaded. These values will be identical for springs with identical force functions. 
Several arguments can be for using identical springs:

1. Identical springs allow the control of position using the difference of muscle activations in a prosthetic application, as shown by equation (4-33).

2. Equation (4-20) shows that the stiffness functions must have the same deflection coefficient and can only differ by a constant. Using a different constant (minimum stiffness from equations (4-18a) and (4-18b)) provides no discernible advantage in the current analysis.

3. Design of the spring functions should include the minimization of internal forces.

Both springs should meet this requirement and thus would be identical. If one spring was able to produce lower internal forces, it would not be $s$. ble to use the other. It would make more sense to use a duplicate of the lower force version.

4. Identical components are easier to manufacture. Only one set of drawings is required and manufacturing procedures remain consistent. This is especially import in mass production.

5. Robotic arms are typically symmetric and operate under the same requirements in both directions. It serves no purpose in such an application to have the two sides different. However, this is not necessarily true for a prosthetic application. The human arm does not work symmetrically about the elbow, both geometrically and in terms of forces. The bicep and tricep sides of the upper arm are built differently. The bicep supports the weight of the arm in most configurations. It is possible that there may be some advantage to designing a prosthesis that simulates this non-symmetry.

If the springs are identical then $x_{1 k}=x_{2 k}$ and equation (4-33) becomes

$$
\theta_{\infty}=\frac{x_{1 a}-x_{2 a}}{2 R}
$$

which allows control of position based on the difference of inputs. As previously mentioned, the stiffness can be controlled by the sum of inputs. This means that the sum and difference technique suggested in Section 3.1.I can be implemented using these 
functions. In addition, the spring stiffnesses can be represented by a single function.

Redefining the minimum and maximum spring stiffnesses and maximum deflection,

$$
\begin{aligned}
& k_{\min }=k_{1 \min }=k_{2 \min } \\
& k_{\max }=k_{1 \max }=k_{2 \max } \\
& \delta_{p 0 x}=\delta_{1 p 0 s}=\delta_{2 p 0 s}
\end{aligned}
$$

By equation (4-21), the general spring function becomes

$$
\psi(\delta\}=\frac{k_{\max }-k_{\operatorname{man}}}{\delta_{\text {pos }}} \cdot \delta+k_{\min }
$$

However, the minimum and maximum spring stiffnesses are not directly specified in manipulation requirements. A better representation is to define the springs based on the joint stiffness requirements. Substitution of equation (4-35a) into equation (4-24) gives the minimum spring stiffness as

$$
k_{\min }=\frac{K_{\min }^{*}}{2 R^{2}}
$$

Likewise, substitution of equation (4-35c) into equation (4-26) gives the deflection coefficient as

$$
A=\frac{K_{\text {max }}^{\bullet}-K_{\text {min }}^{\cdot}}{2 R^{2} \delta_{\text {mas }}}
$$

Substituting equations (4-37) and (4-38) into equation (4-36), the spring function is

$$
\psi\{\delta\}=\frac{K_{m a x}^{*}-K_{m i n}^{*}}{2 R^{2} \delta_{p o s}} \cdot \delta+\frac{K_{m i n}^{*}}{2 R^{2}}
$$

Noting that $x_{1 k}=x_{2 k}$, these values can also be redefined as

$$
x_{\mathrm{L}}=\mathrm{x}_{1 \mathrm{k}}=\mathrm{x}_{2 \mathrm{k}}
$$

Combining equations (4-29) and (4-40), the joint stiffness is

$$
K^{*}=\frac{K_{\max }^{*}-K_{\min }^{*}}{\delta_{\operatorname{mos}}} \cdot x_{1}+K_{\min }^{*}
$$




\subsubsection{Force Function}

Equation (4-39) defines the springs in terms of the desired stiffness change with deflection. It is more typical, however, to define springs by their force-deflection curve. This is also necessary in order to analyze the forces and torques within the actuation system. From equations (3-14a) and (3-14b),

$$
\phi\{\delta\}=\int_{\delta_{0}}^{\delta} \psi\{\delta\} \cdot d \delta
$$

where $\phi\{\delta\}$ is the spring force function. Integrating equation (4-39) (with no initial deflection),

$$
\phi\{\delta\}=\frac{K_{\max }^{\cdot}-K_{\min }^{\cdot}}{4 R^{2} \delta_{\text {pos }}} \cdot \delta^{2}+\frac{K_{\min }^{\cdot}}{2 R^{2}} \cdot \delta+C
$$

where $C$ is the constant of integration. This function has a defined shape as shown in Figure 4.3(a). Note that $\delta_{\text {neg }}$ is defined as the maximum negative deflection of the spring The value of $C$ changes the location of the shape on the vertical axis. For any given $\delta$ the maximum force depends on $\mathrm{C}$. However, the range of force is not a function of $\mathrm{C}$. It is desirable to have minimum internal forces in the mechanism in order to minimize stress and wearing of parts. This can be accomplished by centering the force curve about the horizontal axis (see Figure 4.3(b)) so that the absolute maximum force (tension or compression) is minimum.

Define

$$
\begin{aligned}
& F_{\max }=\text { maximum force } \\
& F_{\min }=\text { minimum force (or maximum negative force) } \\
& F_{\text {mange }}=F_{\max }-F_{\min }=\text { range of force }
\end{aligned}
$$




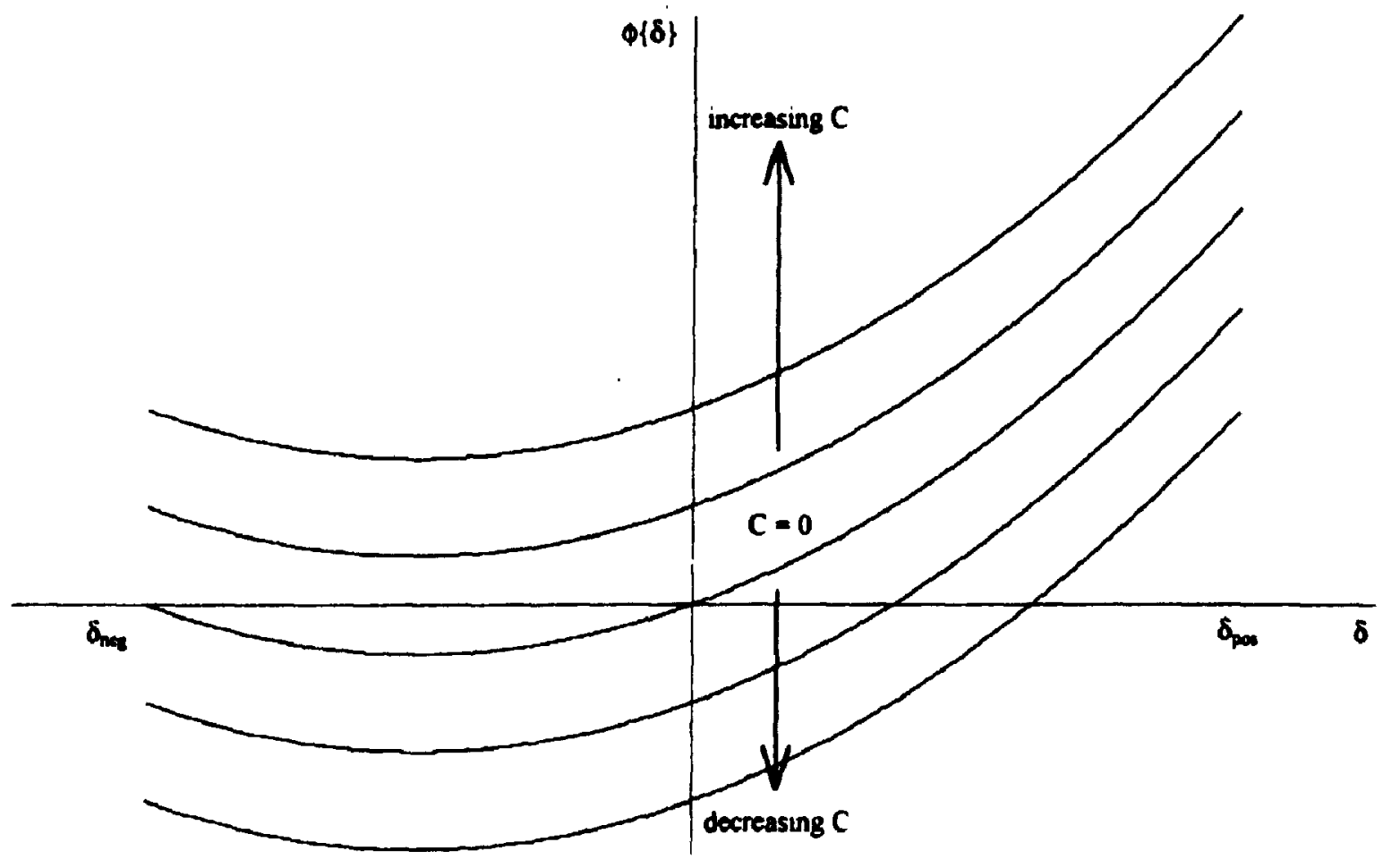

(a)

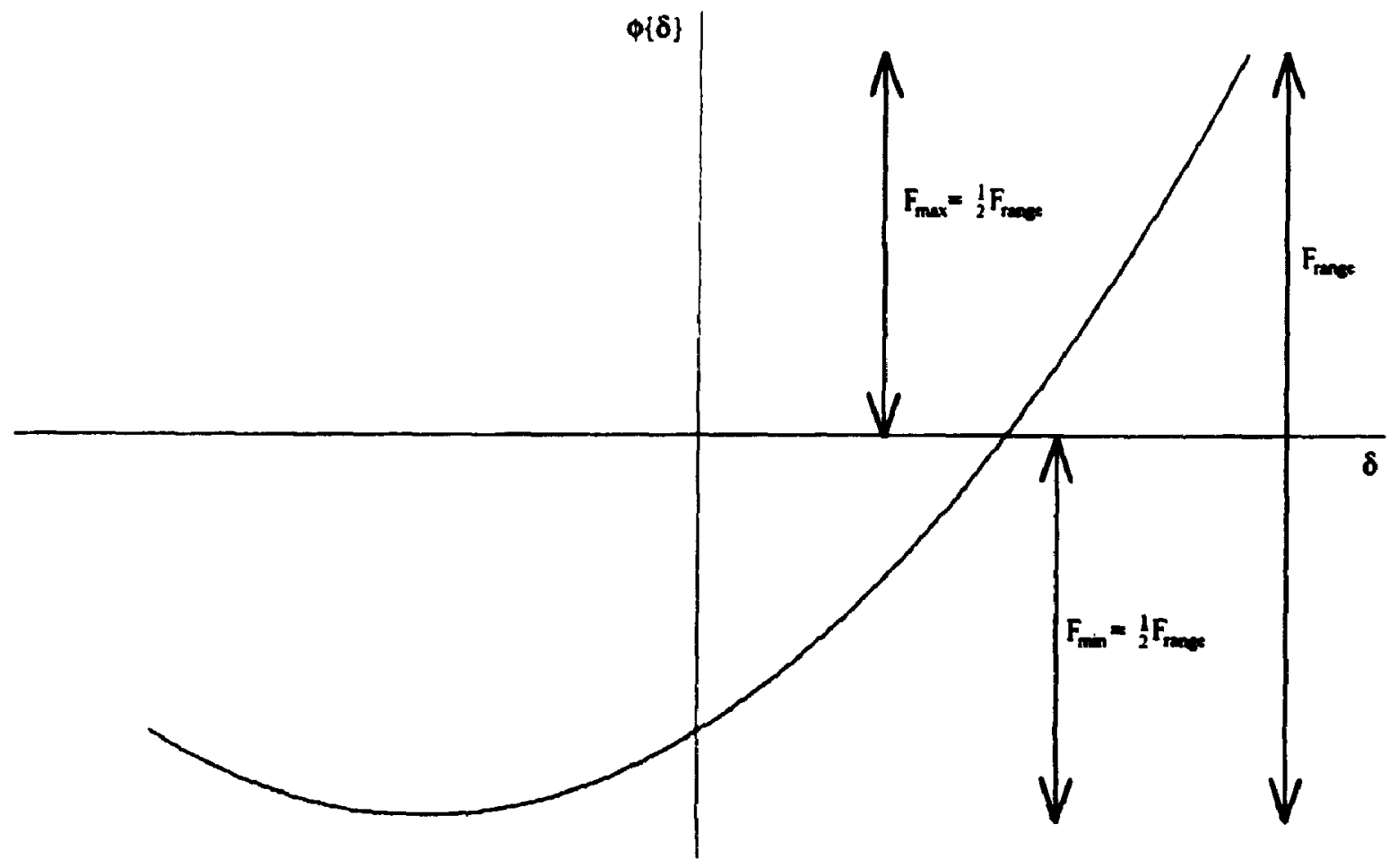

(b)

Figure 4.3. Spring force. (a) variation with $C_{1}$ (b) minimizing the absolute maximum forces. 
From Figure 4.3(a), maximum force occurs at maximum deflection. Maximum deflection in equation (4-42) results in

$$
F_{\text {max }}=\phi\left\{\delta_{\text {pos }}\right\}=\frac{K_{\text {max }}^{*}+K_{\text {min }}^{\cdot}}{4 R^{2}} \cdot \delta_{\text {pos }}+C
$$

$F_{\min }$ is the force at the point where the slope is zero (see Figure 4.3), i.e.,

$$
\frac{d \phi}{d \delta}=0
$$

which, by equations (3-14a) and (3-14b), means $\psi\{\delta\}=0$. Setting equation (4-39) equal to zero and rearranging,

$$
\delta=-\frac{\mathrm{K}_{\min }^{\cdot}}{\mathrm{K}_{\max }^{*}-\mathrm{K}_{\min }^{*}} \cdot \delta_{\text {pos }}
$$

Substituting equation (4-45) into equation (4-42) and simplifying,

$$
F_{\min }=-\frac{K_{\min }^{* 2} \cdot \delta_{p 0 s}}{7 R^{2}\left(K_{\max }^{\circ}-K_{\min }^{*}\right)}+C
$$

Replacing $F_{\max }$ and $F_{\operatorname{man}}$ in equation (4-43) with equations (4-44) and (4-46) and simplifying,

$$
F_{\text {range }}=\frac{\delta_{\text {pos }}}{4 R^{2}} \cdot\left(K_{\text {max }}^{*}+K_{\text {min }}^{*}+\frac{K_{\min }^{\cdot 2}}{K_{\text {max }}^{*}-K_{\min }^{*}}\right)
$$

Centering the force on the horizontal axis requires both maximum and minimum (maximum negative) to be each equal to half of the force range in magnitude, as shown in Figure 4.3(b). Therefore,

$$
F_{\text {max }}=-F_{\text {min }}=1 / 2 F_{\text {range }}
$$

Replacing $F_{\operatorname{mex}}$ and $F_{\text {repse }}$ in equation (4-48) with equations (4-44) and (4-47), respectively, and solving for $C$,

$$
C=-\frac{\delta_{p o s}}{8 R^{2}} \cdot \frac{K_{\max }^{\cdot 2}-2 K_{\min }^{\bullet 2}}{K_{\max }^{*}-K_{\min }^{\circ}}
$$


Following the same substitution in equation (4-48) with $\mathrm{F}_{\min }$ from equation (4-46) results in the same value for $C$. Replacing $C$ in equation (4-42), the spring force is

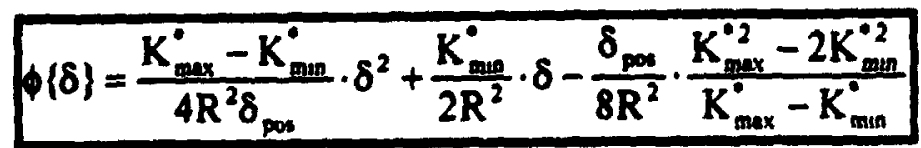

As a check, substitution of equation (4-50) into equation (3-10), using $\delta_{1}$ and $\delta_{2}$ from equations (3.7a) and (3.7b), and simplifying, gives the torque as

$$
T_{e x t}=\left(\frac{K_{\max }^{*}-K_{\min }^{*}}{\delta_{p o s}} \cdot x_{k}+K_{\min }^{\cdot}\right) \cdot \Delta=K^{*} \Delta
$$

which confirms equation (4-9), and shows the linear torque-deflection relationship at the joint.

In summary, the individual spring functions are defined by the force-deflection relation of equation (4-50) and the corresponding stiffness-deflection relation of equation (4-39). The joint stiffness, as a function of $x_{k}$, is given by equation (4-41). Joint equilibrium position, as a function of $x_{\text {mose }}$ is given by equation (4-31). Substitution of equations (4-30) into equation (4-41) gives the joint stiffness as a function of the sum of inputs, whereas equation (4-34) gives joint position as a function of the difference of inputs. Finally, the static joint torque is given by equation (4-51).

Figure 4.4 shows how the quadratic springs combine at the joint to produce a linear spring with a variable stiffness. Figure 4.4(a) shows the combination of spring forces at rest. Note that positive force is defined as one that causes positive torque at the joint according to Figure 3.10. Thus the force curve ior spring two is mirrored about horizontal axis. Following equations (3-7a), (3-7b), and (3-5), $R \theta$ affects $\delta_{1}$, and thus $\phi_{1}$, 
negatively and $\delta_{2}$ and $\phi_{2}$ positively. So positive changes to spring I work in the negative $\mathbf{R} \theta$ direction, while spring $\mathbf{2}$ works in the positive $\mathbf{R} \theta$ direction.

The combination of force functions of the individual springs is a straight line as shown in Figure 4.4(a). Since the springs are at rest position, the stiffness is a minimum. The operating point on the straight line depends upon the deflection of the arm. In this example it is at rest so there is no deflection and the operating point is at the origin. It is also notable that the vertices of the parabolas for the quadratic functions do not fall on the vertical axis for $\mathrm{K}_{\min }^{\circ} \neq 0$, as shown in Figure 4.3. This is why the vertices show an offset for $x_{1 \mathrm{a}}=x_{2 \mathrm{a}}=0$ in Figure 4.4(a).

A first task to examine is a positive endpoint deflection from rest. This intuitively results with a joint torque proportional to minimum stiffness. Figure $4.4(\mathrm{~b})$ shows the graphical results of this task. Since $x_{1}$ and $x_{2 a}$ remain constant, the quadratic curves and straight line summation do not change. However, the deflection of both springs has changed causing a change in the operating point on the linear summation curve. This results in a net force at the joint that, when multiplied by the joint radius, is equivalent to the applied torque $(T=R F)$.

A positive change in equilibrium position from rest is shown in Figure 4.4(c). Both inputs $\left(x_{1,}\right.$ and $\left.x_{2 a}\right)$ are changed by the same amount. This causes the curves to both shift equally along the horizonta! axis. Note that the linear summation keeps the same slope (stiffness) but is also shifted by the same amount. In this task the actual position of the arm, $R \theta$, has changed identically with the equilibrium position. This means that no torque should result since there is no endpoint deflection. Figure 4.4(c) reflects this situation since no net force is created. If the actual a...1 positior ::ad been obstructed from moving as the equilibrium position changed, the operation point would remain at $R \theta=0$ (vertical axis), which on the linear summation curve $\left(\phi_{1}+\phi_{2}\right)$ results in a negative net force. This corresponds to the negative torque that one would expect from such an occurrence. 
A final task to observe is the changing of stiffness from rest. In this case, one would expect that no torque should result without a given endpoint deflection. However, a given deflect ${ }^{*}$ ion should cause higher torques at higher stiffness than observed in Figure 4.4(c). The graphical results of this task can be seen in Figure 4.4(d). Because of the negative influence of $R \theta$ on $\phi_{1}$, the curve for spring 1 is shifted in the negative direction. The positive relationship between $\mathbf{R} \theta$ and spring 2 shifts it in the positive direction. The summation of forces remains linear, but the slope has increased. This represents the increase in stiffness. Note that for the same R $\Delta$ of Figure 4.4(b), the resulting net force would be much higher, as expected from the higher stiffness.

68 
$x_{20}=0$

$\min$ stifiness

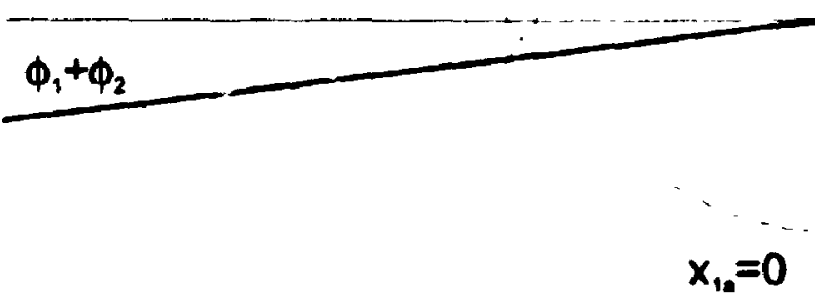

operating

RO

point

$\phi_{2}$

(a)

$\mathbf{F}$

$\phi$

$x_{20}=0$

min. stiffness

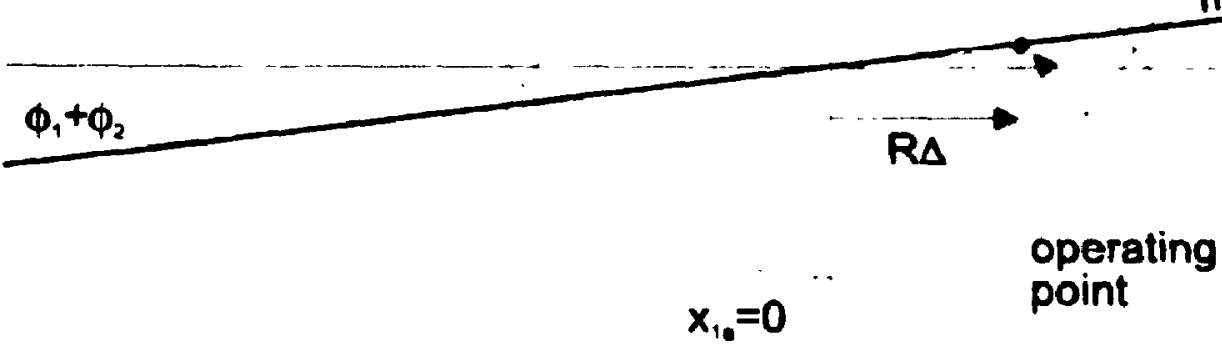

$\phi_{2}$

(b)

Figure 44 Operation of the arm, (a) summation of forces at rest, (b) deflection of endpoint 


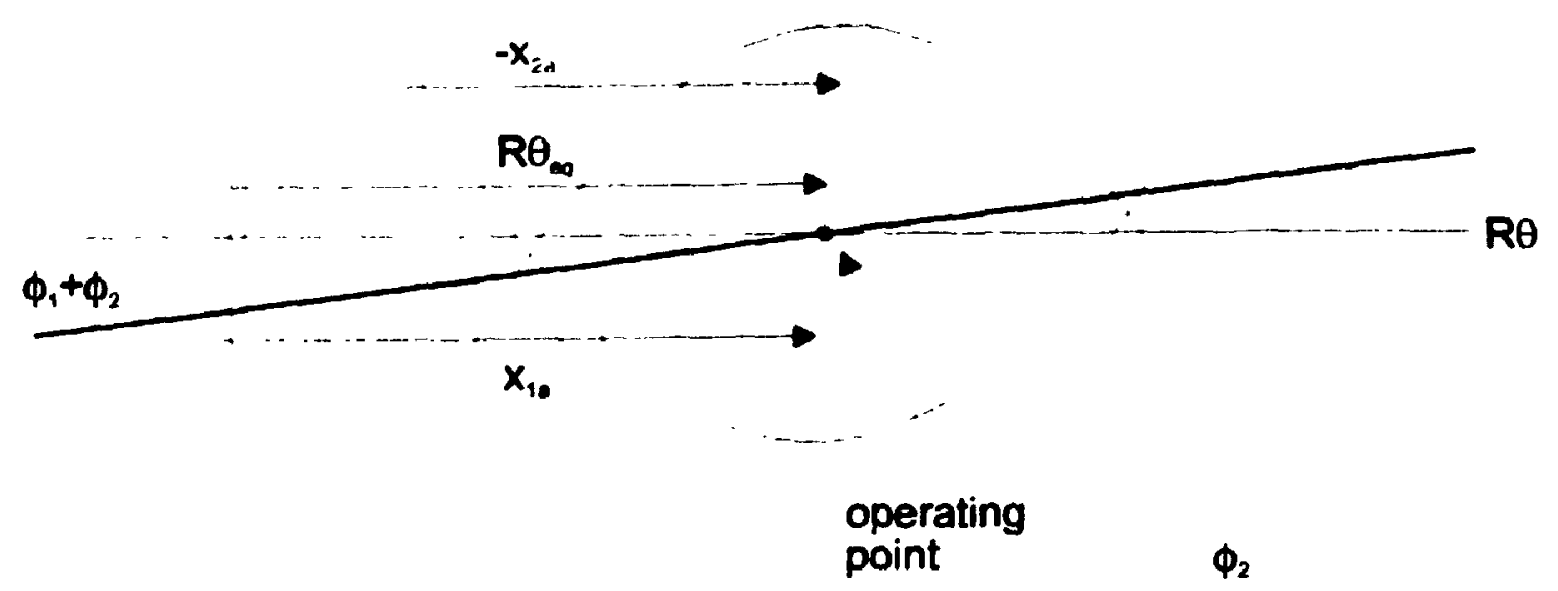

(c)

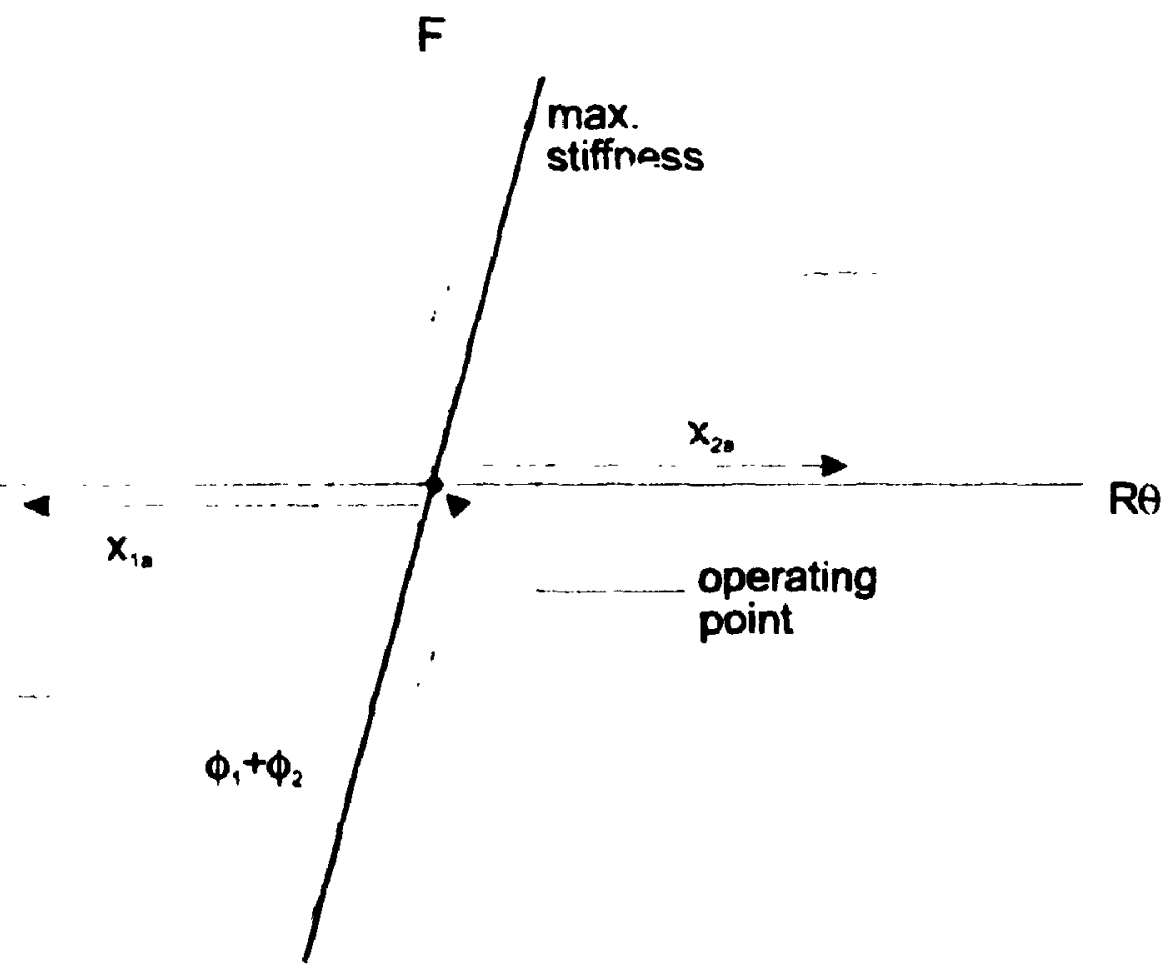

(d)

Figure 44 Operation of the arm, (c) change in equilibrium position, (d) change in stiffness to maximum 


\subsection{Geometric Limitations}

One of the drawbacks of passive impedance control is that physical components are subject to physical limitations, such as the maximum stretch of a spring, and unmodeled characteristics, such as friction forces. This section deals with the former of the two problems, namely the performance limitations of the joint due to physical limitations of the components.

\subsubsection{Spring Limitations}

The only restriction that has been placed upon the springs to this point is the maximum displacement, $\delta_{\text {pos }}$. A brief mention of the maximum negative displacement, $\delta_{\text {neg }}$, was presented in the last section in Section 3.2.3. Figure 4.5 shows both of these restrictions in terms of the spring length. Both are measured from the rest position of the spring, $\delta=0$. The length of the spring at rest is $I_{\text {resi }}$. At maximum negative displacement the remaining length of spring is $1_{\text {min }}$. From Figure 4.5 it can be seen that

$$
1_{\text {resi }}=1_{\text {min }}+\delta_{\text {nep }}
$$

The geometric limitations of the system depend upon the configuration. Antagonistic actuation has typically been implemented using identical dual actuators acting about the joint (Figure 4.6). This is often necessary because the actuation element is combined with the compliant element, such as in a pneumatic bladder. The separation of actuator and spring, as in this design, allows implementation using a "stiffness" actuator and a "position" actuator (Figure 4.7). The latter configuration is especially applicable to the separation of stiffness and motion components $\left(x_{k}\right.$ and $\left.x_{\text {mure }}\right)$ as one actuator can control each variable directly. The physical limitations of each configuration are presented below. 


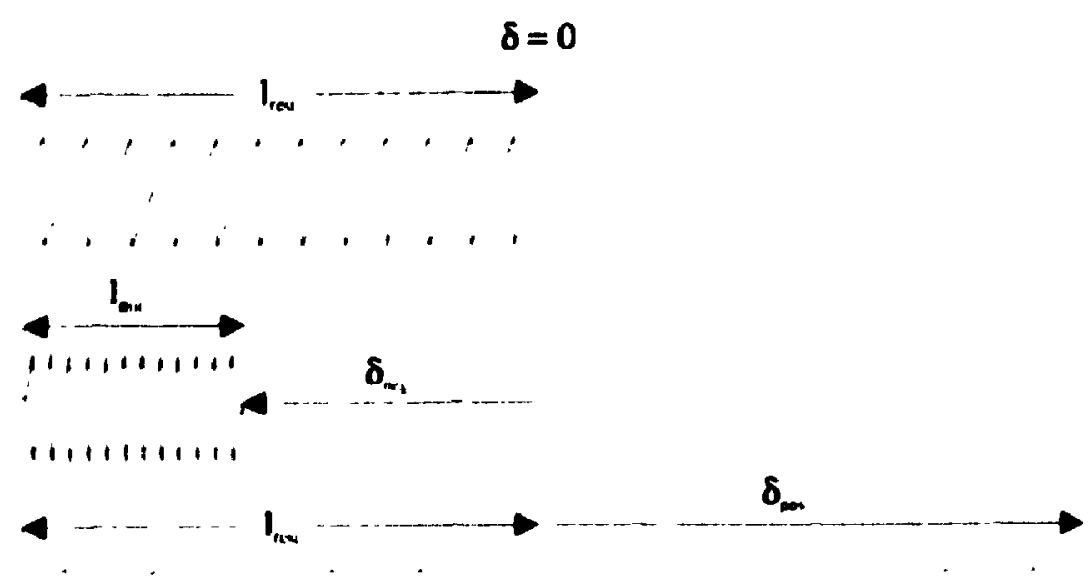

Figure 45 Definition of spring stretch limitations

\section{Configuration 1: Identical Dual Actuators}

In this configuration the actuators and joint are a fixed distance apart, labelled $I_{\operatorname{mav}}$ in Figure 4.6. Figure 4.6(a) shows the system in rest position. From the figure,

$$
A_{0}+B_{0}+I_{\text {rest }}=C_{0}+D_{0}+I_{\text {rest }}=I_{\text {max }}
$$

Figure 4.6(b) shows the system in an activated condition such that the stiffness has increased with no change in equilibrium position. Because there is no position change, there is no $x_{\text {mox }}$ component in $x_{1}$ or $x_{2}$. By equations (3-2a) and (3-2b), and noting the equality of equation (4-40), the input activations for this situation are

$$
x_{1}=x_{1}=x_{2}
$$

Thus the length between actuator and spring in Figure 4.6(b) become

$$
\begin{aligned}
& A_{0}-x_{1}=A_{0}-x_{1} \\
& D_{0}-x_{2}=D_{0}-x_{k}
\end{aligned}
$$

The equilibrium position can only be changed in either direction until one of the springs reaches a physical limit, namely the branch actuator or the joint. Thus the limit for equilibrium rotation in the positive $\theta$ direction is $x_{\text {move }} \leq A_{0}-x_{k}$ or $R \theta \leq C_{0}$, whichever is smaller. If $A_{0}>C_{0}$, but there is some $x_{k}$ at which point $A_{0}-x_{k}<C_{0}$, then the 


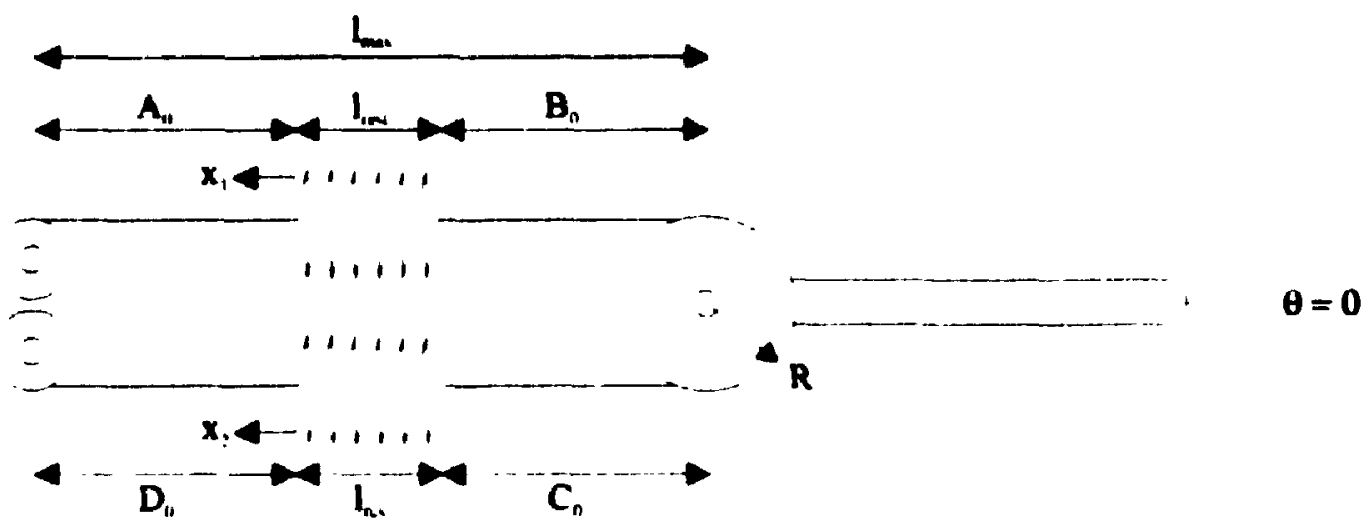

(a)
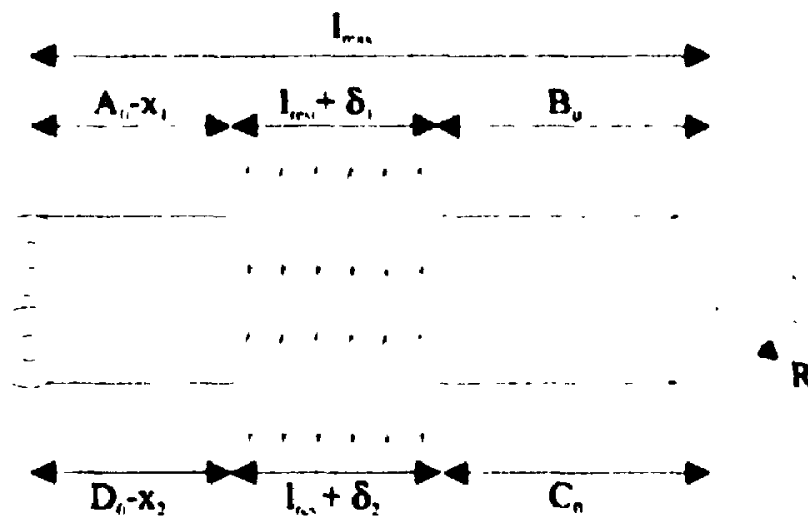

$\theta=0$

(b)

Figure 4.6. Identical dual actuator configuration, (a) at rest, (b) with stiffiness change

equilibrium position limit becomes a function of stiffness. There is no discemible purpose in limiting the range of position for higher stiffnesses, but it does reduce the performance of the joint. Defining $x_{\mathrm{kmax}}$ as the value of $\mathrm{x}_{\mathrm{k}}$ corresponding to maximum joint stiffness, $\mathrm{K}_{\max }$, the system should be designed such that $\mathrm{C}_{0} \geq \mathrm{A}_{0}-\mathrm{x}_{\mathrm{kmax}}$. However, designing $C_{0}>A_{0}-x_{k m a x}$ merely occupies more space with no performance improvements In fact, if space for the actuation system is fixed, such as in a prosthesis, such a condition can diminish performance by requiring other components to be smaller. It is therefore optimum to design the lengths such that $C_{0}=A_{0}-x_{k m a x}$. The arm position in the positive 
direction is then limited by $R \theta \leq A_{0}-x_{k \max }=C_{0}$. Similar arguments can be made for the negative $\theta$ direction such that $R \theta \leq D_{0}-x_{k \max }=B_{0}$.

The total limitation for the arm position is the sum of the positive and negative limits. Using the lengths of branch 1 , this limitation is given by

$$
R \theta_{l a m}=A_{0}-x_{k \max }+B_{0}
$$

where $\theta_{\mathrm{lum}}$ is the range of motion. The parameters used in (4-55) are unique to this limitation analysis. To determine the effects of this limitation on joint performance, it is necessary to relate the limit to previously developed parameters. Noting that $\mathbf{x}_{\mathrm{k}}$ is limited to the maximum stretch of the spring,

$$
x_{1 \text { max }}=\delta_{\text {pos }}
$$

This relationship can also be verified by setting equation (4-41) to maximum stiffness.

The initial lengths $A_{0}$ and $B_{0}$ can be related to the spring and overall length Rearranging equation (4-53),

$$
A_{0}+B_{0}=I_{\text {ma }}-I_{\text {rest }}
$$

The maximum length, $1_{\max }$, depends upon the space available for the application and is thus a free parameter. The rest length can be substituted from equation (4-52). Equation (4-57) then becomes

$$
A_{0}+B_{0}=1_{\max }-1_{\min }-\delta_{\text {nce }}
$$

The minimum spring length, $l_{\min }$, depends upon the spring used. However, since both $I_{\max }$ and $I_{\min }$ are fixed lengths, the combination can be defined as

$$
l_{\text {lim }}=!_{\max }-l_{\min }
$$

Substituting $l_{\text {um }}$, equation (4-56), and equation (4-58) into equation (4-55), the limiting range of motion becomes

$$
R \theta_{\mathrm{llm}}=I_{\mathrm{llm}}-\left(\delta_{\mathrm{pos}}+\delta_{\mathrm{neq}}\right)
$$




\section{Configuration 2: Stiffness and Position Actuation}

This configuration separates the control of stiffness and position to individual actuators. The joint and the stiffness actuator are still a fixed distance apart, but the position actuator must slide horizontally as the stiffness changes, as shown in Figure 4.7. Variation of the stiffness actuator position, $\mathbf{x}_{\mathbf{k}}$, directly stretches both branches by the same amount, unlike the first configuration where each actuator must be separately commanded to move by the same amount. Because the two branches are directly connected around the position actuator, the actuator can control the $x_{\text {manc }}$ of both branches directly by the relation

$$
\varphi_{\text {move }}=\frac{X_{\text {mole }}}{R}
$$

where $\varphi_{\operatorname{mmx}}$ is the rotation of the position actuator from rest. Comparing equation (4-61) with equation (3-1), it can be seen that

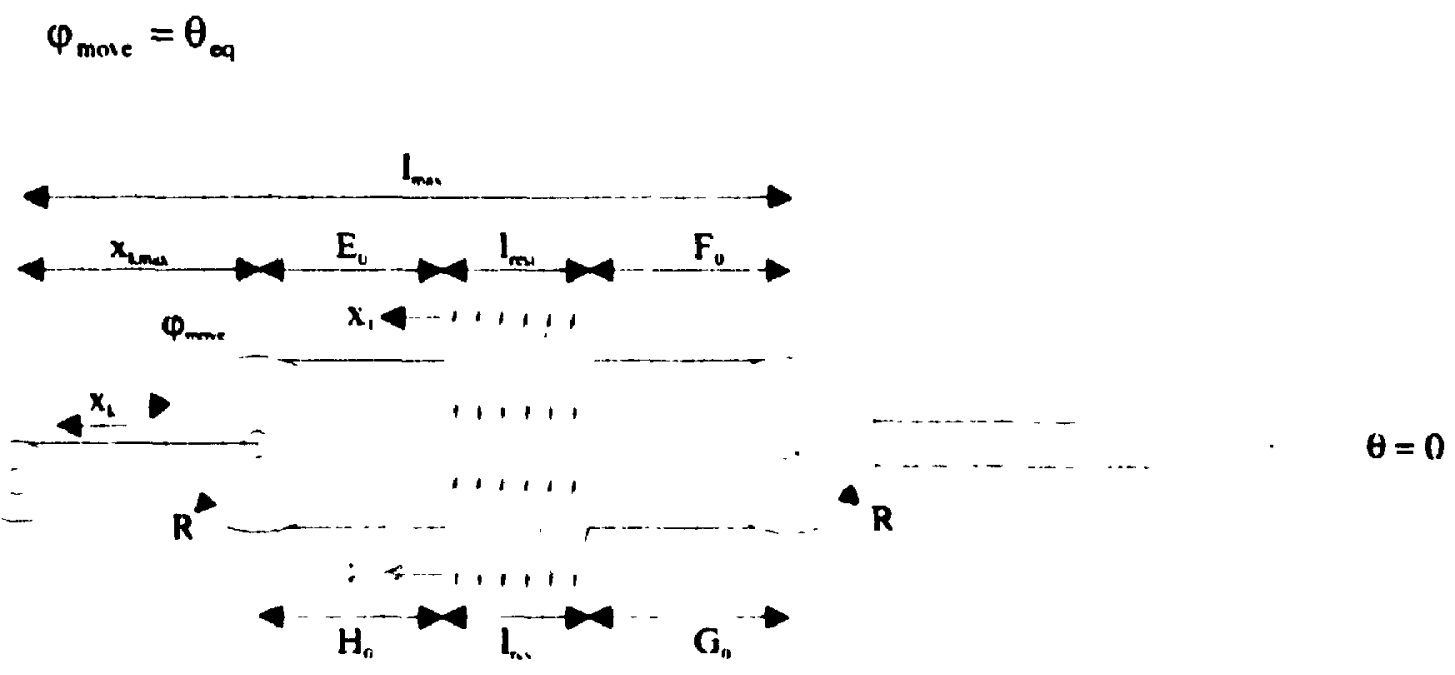

Figure 4.7 Stiffness and position configuration at rest

The main gcometric difference with this configuration is the fact that the lengths between the position actuator and springs are not affected by changes in stiffness. The equilibrium position in the positive $\theta$ direction is limited by the smaller of $E_{0}$ or $G_{00}$. As 
earlier, it serves no purpose to design one of these lengths longer than the other.

Therefore the system should be designed such that $E_{0}=G_{0}$. Similarly, the equilibrium position is limited in the negative direction by $\mathrm{F}_{0}$ or $\mathrm{H}_{0}$, which should be equal by analogy. Using branch 1 lengths, the total range of equilibrium position is

$$
\boldsymbol{R} \boldsymbol{\theta}_{\mathrm{lm}}=\mathrm{E}_{0}+\mathrm{F}_{0}
$$

From the figure,

$$
E_{0}+F_{0}+x_{k \text { max }}+l_{\text {rest }}=G_{0}+H_{0}+x_{k_{\text {max }}}+l_{\text {rost }}=I_{\text {max }}
$$

Rearranging equation (4-63) and substituting into equation (4-62),

$$
R \theta_{l, m}=I_{\text {max }}-I_{\text {reat }}-x_{k \text { max }}
$$

Replacing these parameters with equations (4-52), (4-56), and (4-59),

$$
R \theta_{\mathrm{llm}}=1_{\mathrm{lun}}-\left(\delta_{\text {pos }}+\delta_{\text {neq }}\right)
$$

which is identical to the limitation of Configuration 1, given by equation (4-60).

The sum in braces in equation (4-60) and (4-65) represents the entire range of stretch of the springs. It appears that it might be desirable to replace this sum with a parameter that represents the stretch range of the springs. However, positive and negative deflections of the springs serve different purposes in operation. Examination of equation (4-41) shows that $x_{1} \geq 0$ for $K^{*}{ }_{\text {in }} \leq K^{\circ} \leq K^{\circ}$ max . By equations (3-7a) and (3-7b), noting equation (4-40), the spring displacements can only be negative due to an endpoint deflection, $\Delta$. This deflection is limited either by one spring reaching its maximum stretch or by the other spring reaching its maximum negative displacement. Figure 4.8 shows the case where both limits have been reached at the same time. Such a case will only occur if $t$ nth springs are initially set halfway between the two limits. 


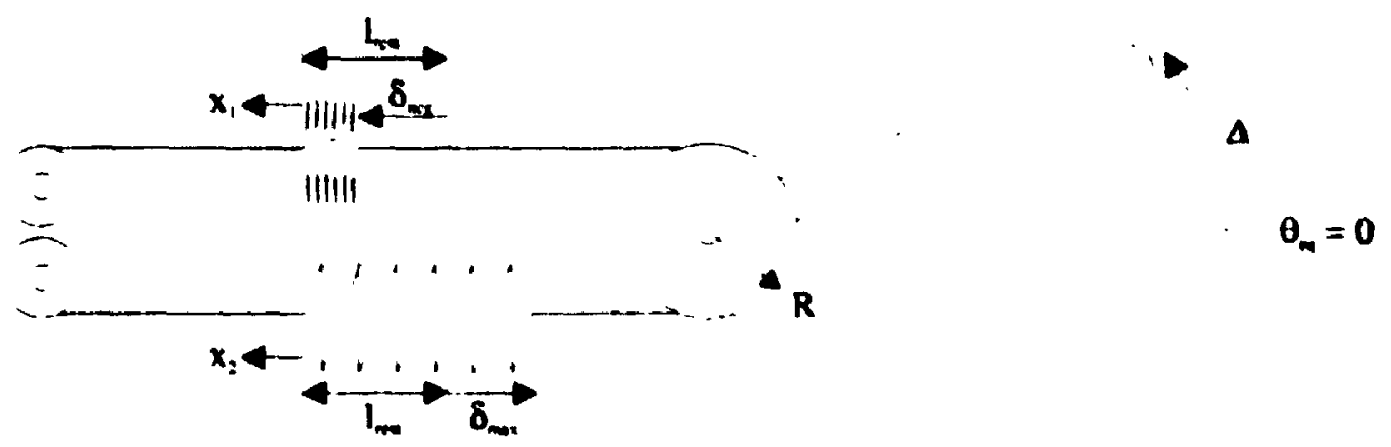

Figure 4.8: Spring limits

At rest the joint stiffness is $\mathrm{K}_{\min }^{\cdot}$ and the springs are at their rest length. If $\delta_{\mathrm{ngg}}=0$, there can be no deflection since one spring would have to displace negatively. If $\delta_{n e q}>\delta_{p o s}$ the joint deflection wor'd be limited by $\delta_{p o s}$, and thus $\delta_{\text {neg }}$ would never be reached. At higher stiffness, and with no joint deflection, the springs are initially stretched and are closer to the $\delta_{p o s}$ limit and so $\delta_{\text {neg }}>\delta_{p p s}$ still provides no useful purpose. Therefore $\delta_{\text {neq }}$ is functionally limited to $0 \leq \delta_{\text {neq }} \leq \delta_{\text {pos. }}$. Rather than leave $\delta_{\text {neq }}$ as a free parameter, this limitation is best depicted as a fraction of $\delta_{\text {pors }}$, such that

$$
\delta_{\text {neq }}=\eta \delta_{\text {pos }}
$$

and $0 \leq \eta \leq 1$. Following this substitution, equation (4-60) (or (4-65)) can be written as

$$
R \theta_{\mathrm{lam}}=I_{\mathrm{llm}}-\delta_{\mathrm{pos}}(1+\eta)
$$

Note that neither limitation analysis of the above two configurations assumes symmetry, i.e., the equilibriurn position is limited by the same amount in the positive and negative directions. This is important in the application to prosthetics where the arm is not limited identically in both directions. 


\subsubsection{Torque Limitations}

This limitation of equation (4-67) by itself does not provide any information as to the performance. However, it does provide a relationship between the operational limitations, the size restrictions, and the spring size. In order to relate these to overall performance, a mathematical representation of performance must be created. The goal of this design is a joint having a linear torque-deflection relationship with a programmable stiffness. This relationship is described by equation (4-9),

$$
\mathrm{T}_{\text {ext }}=\mathrm{K}^{*} \Delta
$$

Limitations on performance can therefore be based on an examination of what boundaries are placed on the above relationship. As mentioned in the previous section, the endpoint deflection, $\Delta$, is limited by the maximum positive and negative spring displacements. As stiffness increases, the springs become initially stretched. This means that there is less spring stretch left that can be applied to an endpoint deflection. From Figure 4.8, the limit of a posittve endpoint deflection occurs either when spring 1 is displaced negatively to a maximum or spring 2 is fully stretched. Substituting the negative displacement limit for spring 1 into equation (3-7a), the relation becomes

$$
-\delta_{\text {neg }}=x_{k}-R \Delta_{\text {lmm }}
$$

where $\Delta_{\mathrm{lmm}}$ is the maximum allowable endpoint deflection. Similarly, substituting the positive displacement limit for spring 2 into equation (3-7b), the relation becomes

$$
\delta_{\text {pos }}=\mathrm{x}_{\mathrm{i}}+\mathrm{R} \Delta_{\mathrm{lim}}
$$

Rearranging equations (4-68) and (4-69), the endpoint displacement is limited by

$$
\begin{aligned}
\Delta_{\mathrm{lum}} & =\frac{x_{k}+\delta_{\text {neg }}}{R} \\
\text { or } \Delta_{\mathrm{lm}} & =\frac{\delta_{\mathrm{por}}-x_{k}}{R}
\end{aligned}
$$


depending on which value is smaller. For any two given values, $M$ and $N$, the smaller one can be determined by the mathematical algorithm

$$
S=\frac{1}{2}(M+N-|M-N|)
$$

where $S$ is the smaller value. Figure 4.9 shows a graphical proof of this algorithm. In the figure, $M$ is smaller than $N$, and the result of the algorithm is the same size as $M$. For $N$ less than $M$, the two letters can simply be switched in the figure to end up with the same result. It is important to note that the difference between values is written as an absolute difference.

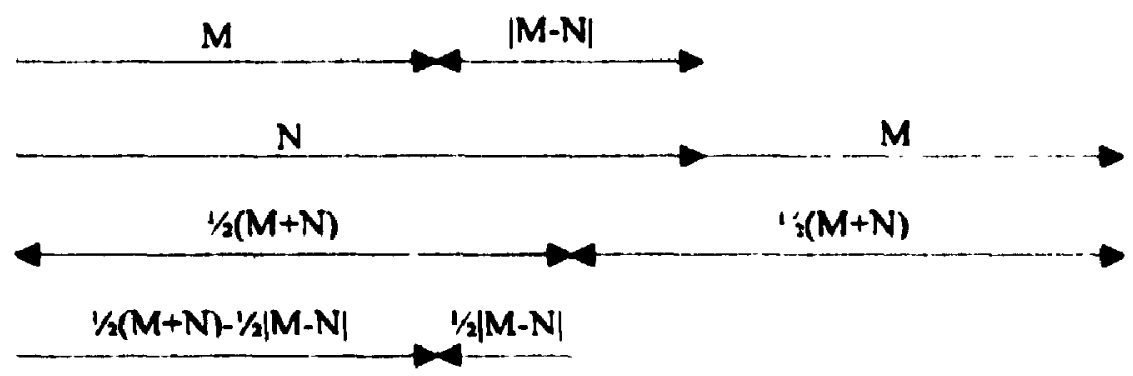

Figure 4.9: Determining the smaller of two values

Using the two possible limitations of endpoint deflection, equations (4-70a) and (4-70b), in the algorithm of equation (4-71), the limit becomes

$$
\Delta_{\text {lum }}=\frac{1}{R}\left[\frac{1}{2}\left(\delta_{\text {pos }}+\delta_{\text {neq }}\right)-\left|x_{k}-\frac{1}{2}\left(\delta_{\text {pos }}-\delta_{\text {neg }}\right)\right|\right]
$$

Replaciñ $\mathcal{S}_{\text {neg }}$ ivith $\eta \delta_{\text {max }}$ as in the previous section,

$$
\Delta_{\text {lim }}=\frac{1}{R}\left[\frac{1}{2} \delta_{\text {pos }}(1+\eta) \cdot \cdot\left|x_{k}-\frac{1}{2} \delta_{p o s}(1-\eta)\right|\right]
$$

The joint stiffness is directly related to $x_{k}$ by equation (4-41). Rearranging to solve 1or $x_{k}$ and substituting into equation (4-72), 


$$
\Delta_{\mathrm{um}}=\frac{\delta_{\text {pon }}}{R}\left[\frac{1}{2}(1+\eta)-\left|\frac{K^{*}-K_{\text {mpp }}^{*}}{K_{\max }^{*}-K_{\min }^{\circ}}-\frac{1}{2}(1-\eta)\right|\right]
$$

assuming $\delta_{\max } \geq 0$. This provides two possible solutions for positive $\Delta_{l_{m}}$, depending upon whether the term within the absolute value is positive or negative. Not all parameters within equations (4-73) can be chosen directly from performance criteria. For instance, there is no direct criterion for selecting the joint radius, $R$. However, the radius and maximum spring stretch, $\delta_{\text {max }}$, are related by equat:on (4-67). Substituting $\delta_{\text {max }}$ from equation (4-67) into (4-73) and simplifying,

$$
\Delta_{\mathrm{hm}}=\left(\frac{l_{\mathrm{lm}}}{2 R}-\frac{1}{2} \mathrm{e}_{\mathrm{lmm}}\right)\left[1-\left|\frac{2\left(\mathrm{~K}^{*}-\mathrm{K}_{\min }^{\cdot}\right)}{\left(\mathrm{K}_{\max }^{*}-\mathrm{K}_{\min }^{*}\right)(1+\eta)}-\frac{1-\eta}{1+\eta}\right|\right]
$$

As well, the criterion for maximum joint torque is related to the maximum force that can be produced in the springs. Therefore, designing the springs based on maximum force rather than maximum stretch is more practical. From equations (4-44) and (4-49), the maximum spring force is

$$
\mathrm{F}_{\max }=\frac{\delta_{\text {pos }}}{8 \mathrm{R}^{2}} \cdot\left(\frac{\mathrm{K}_{\max }^{\cdot 2}}{\mathrm{~K}_{\max }^{*}-\mathrm{K}_{\min }^{\cdot}}\right)
$$

Since $\delta_{\max }$ has already been replaced in equation (4-74), equation (4-75) can be solved for the joint radius for substitution. Once again substituting $\delta_{\max }$ from equation (4-67), equation (4-75) becomes

$$
F_{\text {max }}=\frac{l_{1 \mathrm{~m}}-R \theta_{\mathrm{lum}}}{8 R^{2}(1+\eta)} \cdot\left(\frac{K_{\max }^{* 2}}{K_{\max }^{*}-K_{\min }^{*}}\right)
$$

Rearranging into quadratic form,

$$
8(1+\eta) \mathrm{F}_{\max } \cdot \frac{K_{\max }^{*}-K_{\min }^{*}}{K_{\max }^{\cdot 2}} R^{2}+\theta_{\mathrm{lum}} R-l_{\mathrm{lmm}}=0
$$

It is important to no:e that all relationships developed from Figure 4.6 and 4.7 assume a positive joint radius. A negative joint radius is possible, meaning that the two branches 
cross over and attach to opposite ends of the joint. However, this would change the limitations, including equation (4-67) and thus (4-76). The radius can be forced positive by representing it as a squared value, i.e.,

$$
R=\rho^{2}
$$

Replacing $R$ in equation (4-76) and solving via the quadratic equation,

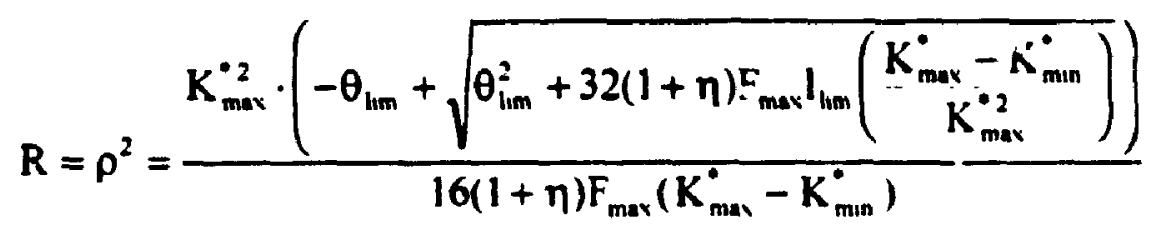

where the positive square root is chosen in order for $p$ to be real. Substituting $R$ from equation (4-77) into (4-74), the endpoint deflection limit becomes

$$
\Delta_{\mathrm{lim}}=\alpha \cdot\left[1-\left|\frac{2\left(\mathrm{~K}^{*}-\mathrm{K}_{\mathrm{m} \cdot \mathrm{n}}^{*}\right)}{\left(\mathrm{K}_{\max }^{*}-\mathrm{K}_{\min }^{*}(1+\eta)\right.}-\frac{1-\eta}{1+\eta}\right|\right]
$$

where

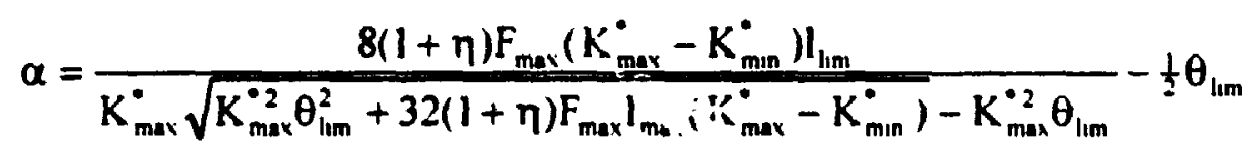

From equation (4-9), the torque required to reach the deflection limit is

$$
\mathrm{T}_{\mathrm{llm}}=\mathrm{K}^{*} \Delta_{\mathrm{llm}}
$$

Equation (4-78) shows that $\Delta_{\mathrm{lmm}}$ is a function of $\mathrm{K}^{*}$. Rearranging to solve for $\mathrm{K}^{*}$ and substitution into equation (4-86) gives the torque ımit as c: function of $\Delta_{\mathrm{lm}}$ and design parameters only. However, there are two solutions for equation (4-78) because of the absolute value. Each case is presented below. 


$$
\frac{2}{m}
$$




\section{Case 1: Positive Value}

In order for the term inside the absolute value to be positive, the condition must be

$$
\frac{1-\eta}{1+\eta}<\frac{2\left(K^{\circ}-K_{\operatorname{mm}}^{\circ}\right)}{(1+\eta)\left(K_{\max }^{\circ}-K_{\min }^{*}\right)} \text { or } \eta>1-\frac{2\left(K^{\circ}-K_{\operatorname{man}}^{\circ}\right)}{\left(K_{\operatorname{man}}^{*}-K_{\operatorname{mn}}^{\circ}\right)}
$$

In this case, rearranging equation (4-78) for $\mathrm{K}^{\circ}$ results in

$$
K^{*}=K_{\operatorname{man}}^{*}-\frac{\left(K_{\operatorname{mx}}^{*}-K_{\operatorname{man}}^{*}\right)(1+\eta) \Delta_{\operatorname{mm}}}{2 \alpha}
$$

Substituting equation (4-81) into equation (4-80), the maximum torque becomes

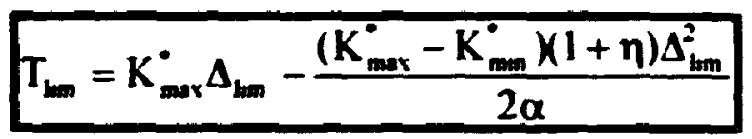

where $\alpha$ is given by equation (4-79). It is important to note that these limitations were derived for positive endpoint deflection only, from equations (4-68) and (4-69).

Substitution for negative deflections follows an almost identical analysis, with the resulting negative torques given by

$$
\mathrm{T}_{\mathrm{lm}}=\frac{\left(\mathrm{K}_{\max }^{\cdot}-\mathrm{K}_{\operatorname{man}}^{\cdot}\right)(1+\eta) \Delta_{\mathrm{lmm}}^{2}}{2 \alpha}-\mathrm{K}_{\max }^{\cdot} \Delta_{\mathrm{lnm}}
$$

Note that equation (4-83) is simply the negative of equation (4-82). This is due to the symmetry of the system.

\section{Case 2: Negative Value}

In order for the term inside the absolute value to be negative, the condition must be

$$
\frac{1-\eta}{1+\eta}>\frac{2\left(K^{*}-K_{\min }^{*}\right)}{(1+\eta)\left(K_{\max }^{*}-K_{\min }^{*}\right)} \text { or } \eta<1-\frac{2\left(K^{*}-K_{\min }^{*}\right)}{\left(K_{\max }^{*}-K_{\min }^{*}\right)}
$$

In this case, rearranging equation (4-78) for $\mathrm{K}^{-}$results in

$$
K^{*}=\frac{\left(K_{\max }^{\cdot}-K_{\min }^{\bullet}\right)(1+\eta) \Delta_{\operatorname{mm}}}{2 \alpha}-\eta K_{\max }^{\cdot}+(1+\eta) K_{\min }^{\cdot}
$$


Substituting equation (4-84) into equation (4-80), the maximum torque becomes

$$
\mathrm{T}_{\mathrm{hm}}=\frac{\left(\mathrm{K}_{\max }^{\prime}-\mathrm{K}_{\min }^{\prime}\right)(1+\eta) \Delta_{\mathrm{hm}}^{2}}{2 \alpha}+\left((1+\eta) \mathrm{K}_{m}^{*}-\eta \mathrm{K}_{\max }^{*}\right) \Delta_{\mathrm{hm}}
$$

where $\alpha$ is given by equation (4-79). The corresponding negative torque is given by

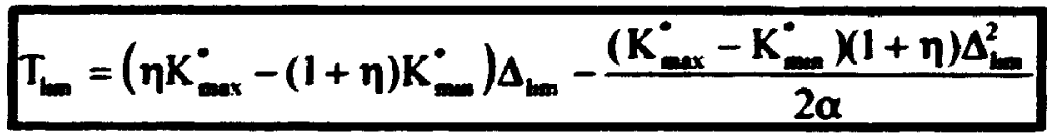

Again the torque limit for negative deflections is the negative of the limit for positive deflections.

Note that an extreme value of $\eta=1$ results in Case 1 for all values of $K^{*}$. This corresponds to springs with identical maximum positive and negative deflections. Values of $\eta$ less than this results in a mixture of the two cases. The combined limits are shown in Figure 4.10(a). The light lines are the bounding torque equations. The dark lines represent the limitations for which each case applies. Case 1 represents the upper limit line. This corresponds to equation (4-70b) where spring 2 reaches its maximum stretch and the system is effectively rigid for further deflections in the positive direction. Similarly, for negative torque the maximum negative limit is reached when spring 1 reaches its maximum stretch and the system is effectively rigid for further deflections in the negative direction. The right-hand limit for positive torque represents Case 2, corresponding to equation (4-70a) where spring 1 reaches its maximum negative deflection and the system is again effectively rigid for further positive joint deflections. Similarty, the left-hand limit for negative torque corresponds to spring $\mathbf{2}$ at its maximum negative deflection. The lower linear limitation for both positive and negative torque represents the minimum design stiffness for the joint.

Figure 4.10 (b) shows the effect of varying $\eta$. From equation (4-66), $\eta$ is the ratio of maximum negative deflection to positive deflection of the springs. Lower values allow for a larger maximum torque but forces a smaller deflection limitation at low stiffness. The allowable torque increases because the smaller springs ( $\eta$ small) allow for 
a larger radius by equation (4-77), assuming $\theta_{\mathrm{m}}$ is kept constant. Thus the maximum force of the springs corresponds to a larger torque. However, allowable joint deflection is reduced because the springs reach maximum negative deflection at smaller joint deflections. For instance, $\eta=0$ means that the springs cannot deflect negatively $(\delta<0)$. Thus at minimum stiffness ( $\delta=0$ for no joint deflection), no joint deflection is possible since it would cause one of the springs to deflect into the negative region. This can be seen for the $\eta=0$ case of Figure $4.10(\mathrm{~b})$.

The ideal operation of a variable stiffness joint allows infinite torque and deflection for any stiffness. For practical applications these lines can be limited to a minimum and maximum stiffiness, as shown in Figure 4.11(a), and maximum torque and deflection values that meet required criteria. The above geometric analysis of the proposed system using mechanical springs results in operating torque and deflection limits as a function of the stiffness. The effect on the operation of the joint is shown in Figure 4.11(b). For torques and deflections within the area defined in Figure 4.10(a), the system operates as a variable stiffness joint. Therefore, this region is termed the variable stiffness region. Beyond these limits the joint is effectively rigid, and any stiffness, labelled $\mathrm{K}_{\mathrm{am}}$ in Figure 4.11(b), is due to material compliance of the arm and components (gears, cable, etc.). This region is therefore termed the rigid region. 
$T_{L}$

$\mathbf{K}^{*}$

Case 2

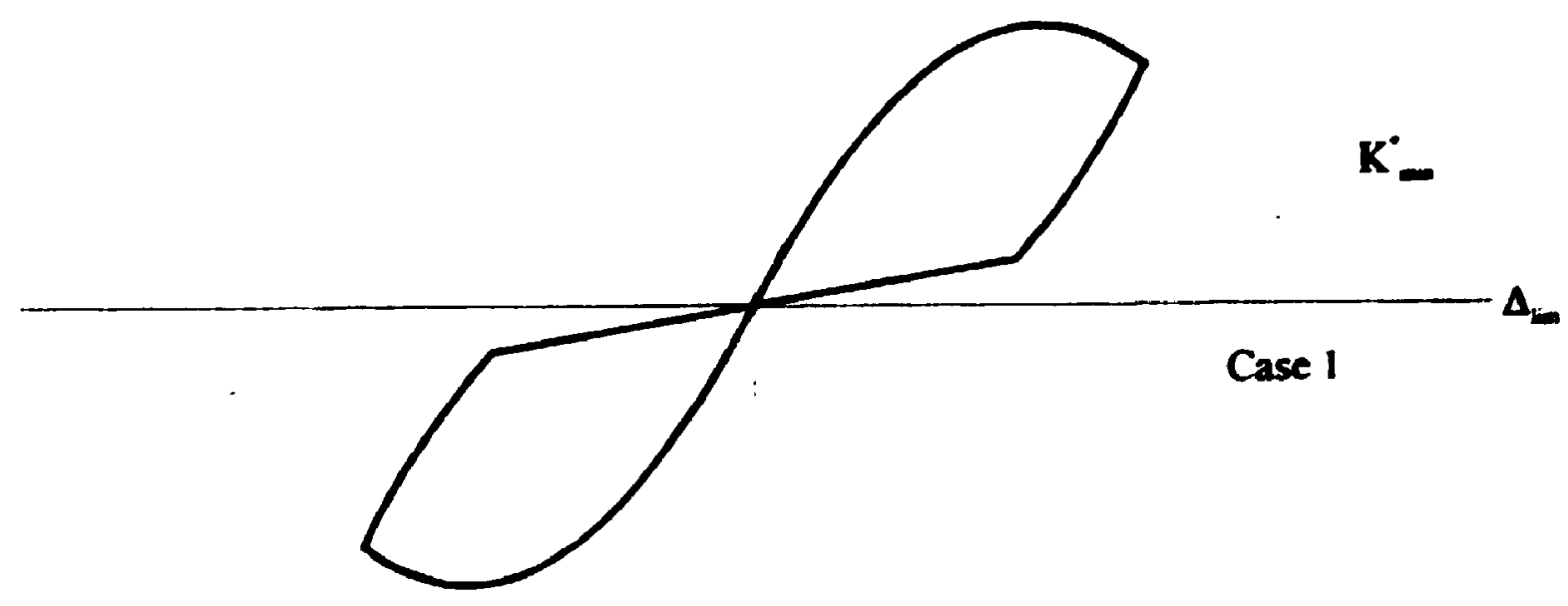

(a)

$T_{\text {I }}$

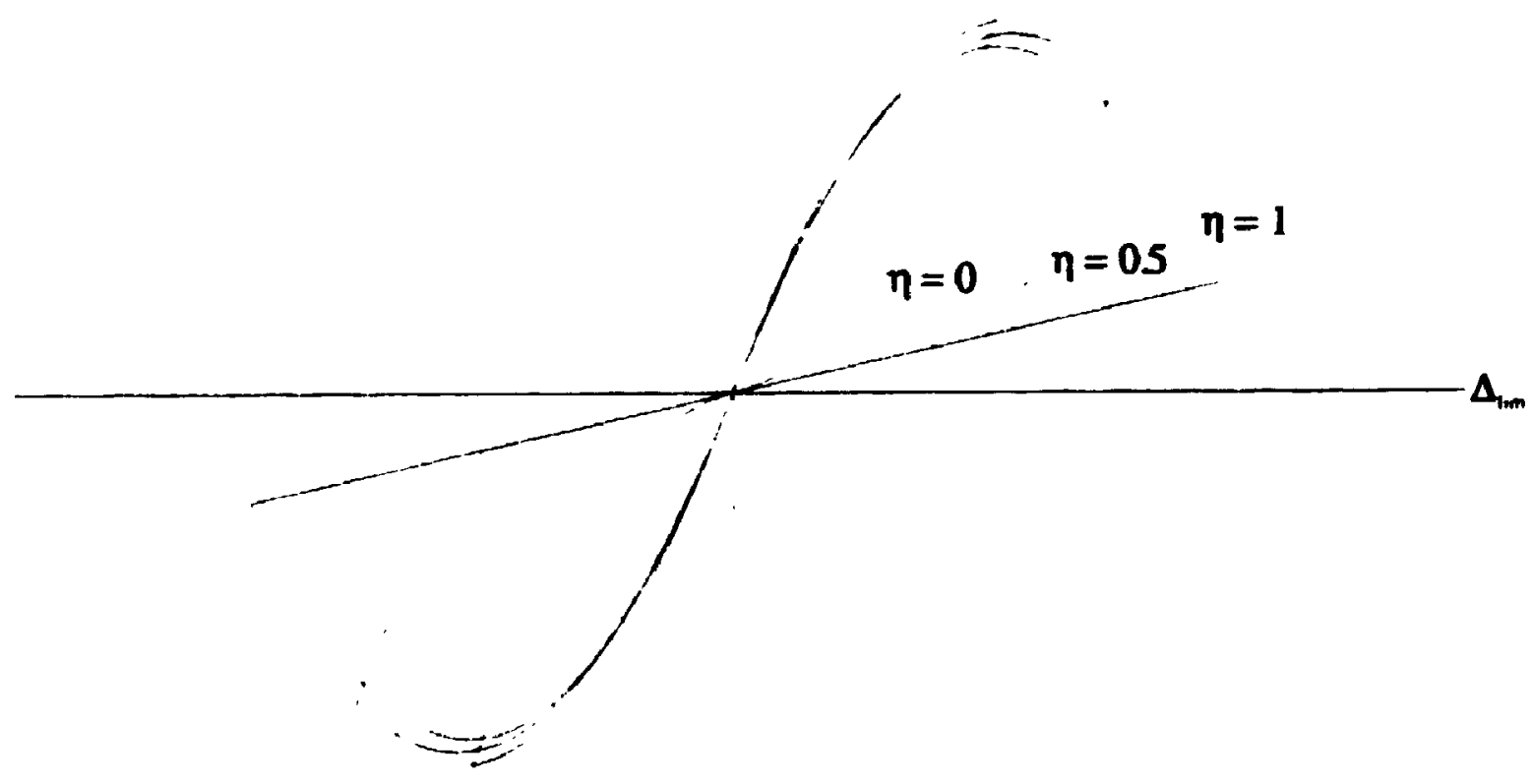

(b)

Figure 4.10: Limiting torque, (a) as function of $\Delta_{\operatorname{man}}$ (b) effect of varying $\eta$.

85 


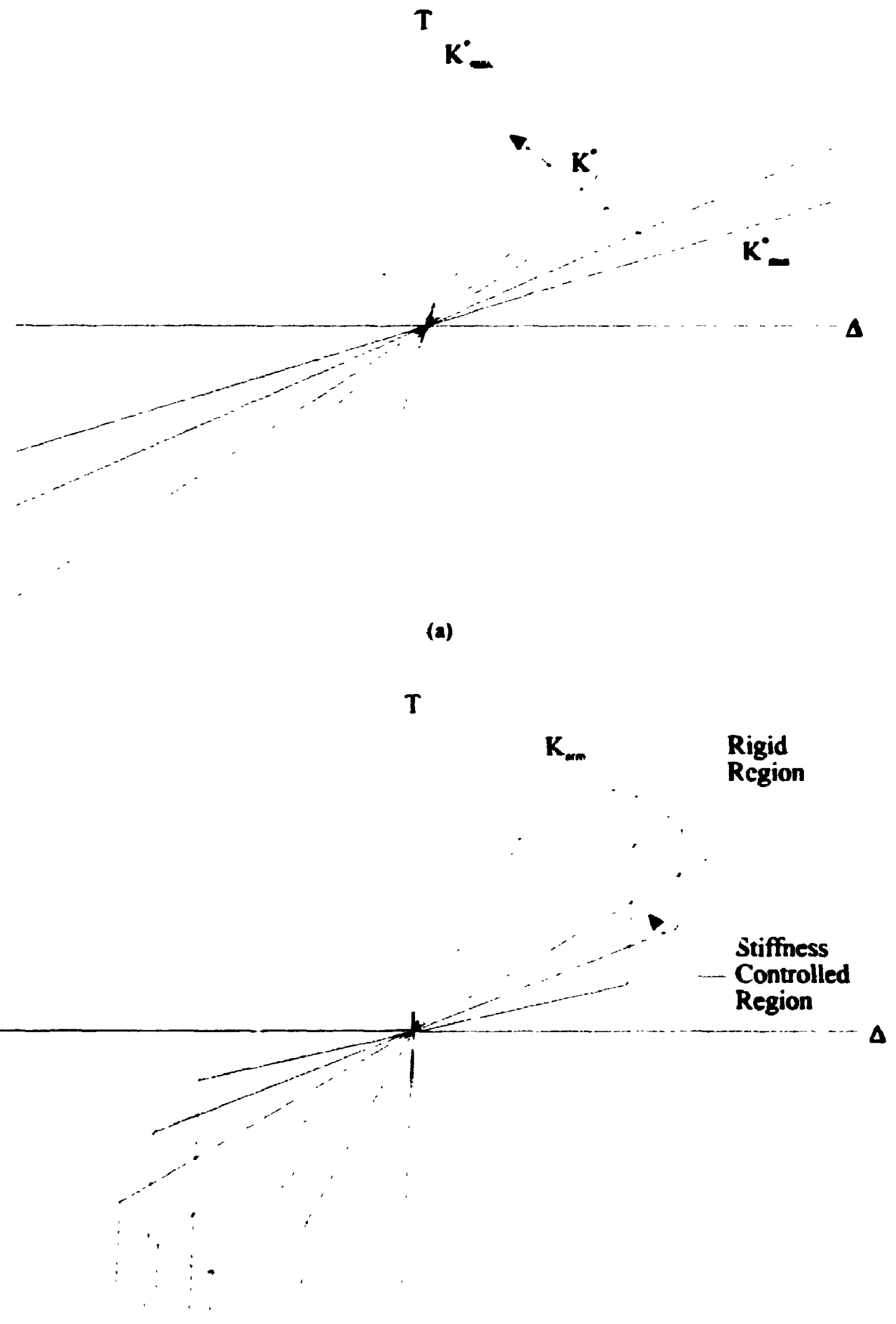

(b)

Figure 4.11: Variable siffiness operation, (a) ideal, (b) with geometric limitations 


\subsection{Non-Linear Stiffness}

The requirement that joint stiffness and position be decoupled requires the use of antagonistic springs with linearly varying stiffness, as shown in Section 4.1.1. This criterion allows control of stiffness in open loop. However, this decoupling is not an absolute necessity for proper performance of the arm. Feedback on the joint can be used to track endpoint deflection This, in turn, can be used in the modulation of stiffness. Such feedback may be necessary in any case to determine the environmental forces. Also, study of other criteria may show performance is optimum for stiffness elements that do no: vary !inearly. Most antagonistic actuation schemes, including the human musculoskeletal system, do not use such a system. It is therefore imperative to perform some analysis of non-linear stiffness elements in antagonistic actuation.

\subsubsection{Stiffness Function}

The joint stiffness for general antagonistic spring functions, given by equation (3-15) using identical springs, is

$$
K^{*}=R^{2} \cdot\left(\psi\left\{\delta_{1}\right\}+\psi\left\{\delta_{2}\right\}\right)
$$

Using the stiffness and position model of Section 3.1.2, the joint stiffness is generally a function of both $x_{t}$ and $\Delta$. This is evident from the definition of spring displacements in equations (3-7a) and (3-7b). In the special case of decoupled stiffness and position, the endpoint deflection is eliminated from the above equation by creating spring functions for which $\Delta$ cancels. Since there are an infinite number of equations that can be developed for the stiffness elements, such as pneumatic bladders, a general analysis cannot be created that encompasses all applications. However, the effect of endpoint deflection on stiffness can be examined. 
The set stiffness is typically defined with no deflection of the endpoint, i.e., $\Delta=0$. In this situation, the spring displacements, from equations (3-7a) and (3-7b), are

$$
\begin{aligned}
& \delta_{1}=\delta_{k}=x_{k} \\
& \delta_{2}=\delta_{k}=x_{k}
\end{aligned}
$$

where $\delta_{\mathrm{k}}$ is defined as the spring displacement caused by a change in stiffness only. Note that it is numerically equivalent to $x_{k}$, but $x_{k}$ is defined as the contribution to the branch inpul caused by a change in stiffness (see Figure 3.9 and equations (3-2a), (3-2b), and subsequently equation (4-40)). Introducing equations (4-88a) and (4-88b) into equation (3-15), the set stiffness of the joint is defined as

$$
K_{x+1}^{\bullet}=2 R^{2} \Psi\left\{\delta_{k}\right\}
$$

The change in joint stiffness due to an endpoint deflection is therefore the difference between the given joint stiffness (equation (4-87)) and the set stiffness (equation (4-89)). Defining this deflection stiffness change as $\mathrm{K}_{\Delta}^{*}$, it is given by

$$
K_{3}^{*}=R^{2} \cdot\left(\psi\left\{\delta_{1}\right\}+\psi\left\{\delta_{2}\right\}-2 \psi_{2}\left\{\delta_{k}\right\}\right)
$$

Normalizing the change in stiffness,

$$
\xi=\frac{K_{j}^{*}}{K_{s}^{*}}=\frac{\Psi\left\{\delta_{1}\right\}+\Psi\left\{\delta_{2}\right\}}{2 \Psi\left\{\delta_{k}\right\}}-1
$$

where $\xi$ is the normalized change in stiffness. For reference purposes, this deviation from set joint stiffness is considered a stiffness error. Thus $\xi$ is a measure of the error in joint stiffness. Rearranging to separate variables,

$$
2(\xi+1) \psi\left\{\delta_{k}\right\}=\psi\left\{\delta_{1}\right\}+\psi\left\{\delta_{2}\right\}
$$

Comparison of equations (4-92) with equations (4-87) and (4-89) reveals

$$
K^{*}=2 R^{2}(\xi+1) \psi\left\{\delta_{k}\right\}=(\xi+1) K_{x+1}^{\circ}
$$

In general, $\xi$ can be a function of both $\delta_{k}$ and $\Delta$. If it is a function of $\delta_{k}$ only, the stiffness is once again independent of $\Delta$ by equation (4-93). From Section 4.1.1, this results in quadratic springs. If the relationship is a function of both parameters, it must be of the 
form $\xi\left\{\delta_{k}, \Delta\right\}=f\left\{\delta_{k}, \Delta\right\} g\{\Delta\}$, where $g\{0\}=0$ since there is no change in stiffness without an endpoint deflection. This case implies that the effect of endpoint deflection on joint stiffness depends upon the set stiffiness. While this is the more general case, the relationship is more clear if the change in stiffness due to endpoint deflections is independent of set stiffiness, i.e., $\xi=\xi\{\Delta\}$. To simplify the mathematical manipulation, define

$$
E\{\Delta\}=2(\xi\{\Delta\}+1)
$$

Substituting this into equation (4-92),

$$
E\{\Delta\} \psi\left\{\delta_{k}\right\}=\psi\left\{\delta_{1}\right\}+\psi\left\{\delta_{2}\right\}
$$

By analogy to Section 4.1.1, the stiffness functions are determined by the characteristics of the slope and curvature of the set joint stiffness as a function of deflection or stiffness change. Equation (4-95) represents the joint stiffness divided by the square of the joint radius (see equation (4-93)). Thus it can be used to examine the effect of changes to set stiffness and endpoint deflection. Taking the derivative with respect to a commanded stiffness change,

$$
E\{\Delta\} \frac{d \psi\left\{\delta_{k}\right\}}{d x_{k}}=\frac{d \psi\left\{\delta_{1}\right\}}{d x_{k}}+\frac{d \psi\left\{\delta_{2}\right\}}{d x_{k}}
$$

By the chain rule,

$$
E\{\Delta\} \frac{d \psi\left\{\delta_{k}\right\}}{d \delta_{k}} \cdot \frac{d \delta_{k}}{d x_{k}}=\frac{\partial \psi\left\{\delta_{k}\right\}}{\partial \delta_{1}} \cdot \frac{d \delta_{1}}{d x_{k}}+\frac{\partial \psi\left\{\delta_{2}\right\}}{\partial \delta_{2}} \cdot \frac{d \delta_{2}}{d x_{k}}
$$

From equation (4-88a) and (4-88b),

$$
\frac{d \delta_{k}}{d x_{k}}=1
$$

Substituting equations (4-5a), (4-5b), and (4-97) into equation (4-96),

$$
E\{\Delta\} \frac{d \psi\left\{\delta_{k}\right\}}{d \delta_{k}}=\frac{\partial \psi\left\{\delta_{1}\right\}}{\partial \delta_{1}}+\frac{\partial \psi\left\{\delta_{2}\right\}}{\partial \delta_{2}}
$$


Likewise, taking the derivative of equation (4-95) with respect to a change in endpoint deflection,

$$
\frac{d E\{\Delta\}}{d \Delta} \psi\left\{\delta_{k}\right\}=\frac{d \psi\left\{\delta_{1}\right\}}{d \Delta}+\frac{d \psi\left\{\delta_{2}\right\}}{d \Delta}
$$

By the chain rule,

$$
\frac{d E\{\Delta\}}{d \Delta} \psi\left\{\delta_{k}\right\}=\frac{\partial \psi\left\{\delta_{1}\right\}}{\partial \delta_{1}} \cdot \frac{d \delta_{1}}{d \Delta}+\frac{\partial \psi\left\{\delta_{2}\right\}}{\partial \delta_{2}} \cdot \frac{d \delta_{2}}{d \Delta}
$$

Substituting equations (3-13a) and (3-13b) and simplifying,

$$
\frac{d E\{\Delta\}}{d \Delta} \psi\left\{\delta_{k}\right\}=R\left(-\frac{\partial \psi\left\{\delta_{1}\right\}}{\partial \delta_{1}}+\frac{\partial \psi\left\{\delta_{2}\right\}}{\partial \delta_{2}}\right)
$$

Unlike the previous analysis in Section 4.1.1, the slopes of the spring stifiness curves do not provide a direct relation. However, equations (4-98) and (4-99) do provide a relationship for the sum and difference of the individual spring stiffness slopes.

Continuing the analogy of Section 4.1.1, the curvatures can be related by the derivatives of equations (4-98) and (4-99) with respect to stiffness and deflection changes.

Beginning with equation (4-98), the derivative with respect to a change in stiffness is

$$
E\{\Delta\} \frac{d}{d x_{k}}\left(\frac{d \psi\left\{\delta_{k}\right\}}{d \delta_{k}}\right)=\frac{\partial}{\partial x_{k}}\left(\frac{\partial \psi\left\{\delta_{1}\right\}}{\partial \delta_{1}}\right)+\frac{\partial}{\partial x_{k}}\left(\frac{\partial \psi\left\{\delta_{2}\right\}}{\partial \delta_{2}}\right)
$$

Using the chain rule and substitution for the $\delta$ 's,

$$
E\{\Delta\} \frac{d^{2} \Psi\left\{\delta_{k}\right\}}{d \delta_{k}^{2}}=\frac{\partial^{2} \psi\left\{\delta_{i}\right\}}{\partial \delta_{1}^{2}}+\frac{\partial^{2} \psi\left\{\delta_{2}\right\}}{\partial \delta_{2}^{2}}
$$

Now taking the derivative of equation (4-98) with respect to a deflection,

$$
\frac{d E[\Delta\}}{d \Delta} \cdot \frac{d \psi\left\{\delta_{k}\right\}}{d \delta_{k}}=\frac{\partial}{\partial \Delta}\left(\frac{\partial \psi\left\{\delta_{1}\right\}}{\partial \delta_{1}}\right)+\frac{\partial}{\partial \Delta}\left(\frac{\partial \psi\left\{\delta_{2}\right\}}{\partial \delta_{2}}\right)
$$

Using the chain rule and substitutions as above, 


$$
\frac{d E\{\Delta\}}{d \Lambda} \cdot \frac{d \psi\left\{\delta_{k}\right\}}{d \delta_{k}}=R\left(-\frac{\partial^{2} \psi\left\{\delta_{1}\right\}}{\partial \delta_{1}^{2}}+\frac{\partial^{2} \psi\left\{\delta_{2}\right\}}{\partial \delta_{2}^{2}}\right)
$$

Next, taking the derivative of equation (4-99) with respect to a stiffness change,

$$
\frac{d E\{\Delta\}}{d \Delta} \frac{d \psi\left\{\delta_{k}\right\}}{d x_{k}}=R\left(-\frac{\partial}{\partial x_{k}}\left(\frac{\partial \psi\left\{\delta_{1}\right\}}{\partial \delta_{1}}\right)+\frac{\partial}{\partial x_{k}}\left(\frac{\partial \psi\left\{\delta_{2}\right\}}{\partial \delta_{2}}\right)\right)
$$

By chain rule and substitution, this simplifies to

$$
\frac{d E\{\Delta\}}{d \Delta} \frac{d \psi\left\{\delta_{k}\right\}}{d \delta_{k}}=R\left(-\frac{\partial^{2} \psi\left\{\delta_{1}\right\}}{\partial \delta_{1}^{2}}+\frac{\partial^{2} \psi\left\{\delta_{2}\right\}}{\partial \delta_{2}^{2}}\right)
$$

Note that equations (4-101) and (4-102) are identical. This shows that the system is conservative, i.c.,

$$
\frac{d}{d \Delta}\left(\frac{d K^{*}}{d x_{k}}\right)=\frac{d}{d x_{k}}\left(\frac{d K^{*}}{d \Delta}\right)
$$

Taking the derivative of equation (4-99) with respect to a deflection change,

$$
\frac{d^{2} E\{\Delta\}}{d \Delta^{2}} \psi\left\{\delta_{k}\right\}=R\left(-\frac{\partial}{\partial \Delta}\left(\frac{\partial \psi\left\{\delta_{1}\right\}}{\partial \delta_{1}}\right)+\frac{\partial}{\partial \Delta}\left(\frac{\partial \psi\left\{\delta_{2}\right\}}{\partial \delta_{2}}\right)\right)
$$

By the chain rule and substitution,

$$
\frac{d^{2} E\{\Delta\}}{d \Delta^{2}} \psi\left\{\delta_{k}\right\}=R^{2}\left(\frac{\partial^{2} \psi\left\{\delta_{1}\right\}}{\partial \delta_{1}^{2}}+\frac{\partial^{2} \psi\left\{\delta_{2}\right\}}{\partial \delta_{2}^{2}}\right)
$$

A comparison of the curvatures of the spring stiffness functions, by equations (4-100) and (4-103) reveals that

$$
\frac{d^{2} E\{\Delta\}}{d \Delta^{2}} \psi\left\{\delta_{k}\right\}=R^{2} E\{\Delta\} \frac{d^{2} \Psi\left\{\delta_{k}\right\}}{d \delta_{k}^{2}}
$$

To simplify the expression, define

$$
E^{n}=\frac{d^{2} E\{\Delta\}}{d \Delta^{2}}
$$

and 


$$
\psi^{\prime \prime}=\frac{d^{2} \Psi\left\{\delta_{k}\right\}}{d \delta_{k}^{2}}
$$

Introducing these notations into equation (4-104) and separating the variables.

$$
\frac{\Psi^{\prime \prime}}{\Psi}=\frac{1}{R^{2}} \cdot \frac{E^{\prime \prime}}{E}=\lambda
$$

where $\lambda$ is a constant since $\delta_{k}$ and $\Delta$ are independent variables. This relationship is a common mathematical problem [Hanna and Rowland, 1990]. Rearranging equation (4107), the functions $\psi\{\delta\}$ and $E\{\Delta\}$ can be found as the solutions to the following linear differential equations:

$$
\begin{aligned}
& \boldsymbol{\psi}^{\prime \prime}-\lambda \psi=0 \\
& E^{\prime \prime}-\lambda R_{e}^{2} E=0
\end{aligned}
$$

The general solutions for these equations are of the form

$$
\begin{aligned}
& \Psi\left\{\delta_{k}\right\}=A \cdot \cosh \left(\sqrt{\lambda} \cdot \delta_{k}+B\right) \\
& E\{\Delta\}=C \cdot \cosh (\sqrt{\lambda} \cdot R \Delta+D)
\end{aligned}
$$

The actual solutions depend on the value of $\lambda$. There are three possibilities: $\lambda=0, \lambda<0$, and $\lambda>0$. Each case is presented below.

Case 1: $\lambda=0$

The solutions are in the form

$$
\begin{aligned}
& \Psi\left\{\delta_{k}\right\}=A_{1} \delta_{k}+B_{1} \\
& E(\Delta)=C_{1} \Delta+D_{1}
\end{aligned}
$$

The boundary conditions on $\Psi\{\delta\}$ are

$$
\psi\{0\}=k_{\min }=\frac{K_{\operatorname{man}}^{*}}{2 R^{2}}=B_{1}
$$

and $\quad \psi\left(\delta_{\operatorname{mos}}\right\}=k_{\max }=\frac{K_{n-x}^{*}}{2 R^{2}}=A_{1} \delta_{\max }+\frac{K_{m a n}^{*}}{2 R^{2}}$

Rearranging, 


$$
A_{1}=\frac{K_{\max }^{*}-K_{m}^{\cdot}}{2 R^{2} \delta_{\text {pox }}}
$$

Substituting these coefficients into equation (4-110), for a general deflection the stiffness function is

$$
\psi\{\delta\}=\frac{K_{\max }^{\circ}-K_{\min }^{\circ}}{2 R^{2} \delta_{\operatorname{mos}}} \delta+\frac{K_{\operatorname{mom}}^{\circ}}{2 R^{2}}
$$

Substituting equations (4-112), (3-7a), (3-7b), (4-88a), and (4-88b) into equation (4-95), and simplifying.

$$
E\{\Delta\}\left(\frac{K_{\max }^{*}-K_{\operatorname{man}}^{*}}{2 R^{2} \delta_{\text {pos }}} x_{k}+\frac{K_{\operatorname{man}}^{\cdot}}{2 R^{2}}\right)=2\left(\frac{K_{m x}^{*}-K_{\max }^{\cdot}}{2 R^{2} \delta_{p o s}} x_{k}+\frac{K_{\operatorname{man}}^{*}}{2 R^{2}}\right)
$$

Therefore

$$
E\{\Delta\}=2
$$

and by equation (4-94)

$$
\xi\{\Delta\}=0
$$

Thus $\lambda=0$ results in the trivial case where the change of joint stiffness due to endpoint deflection is always zero. A comparison of equation (4-112) to equation (4-39) confirms the earlier results for independent stiffness and position.

Case 2: $\lambda>0$

To force $\lambda$ positive, let $\lambda=\mu^{2}$. The solutions are then of the form

$$
\begin{aligned}
& \psi\left\{\delta_{k}\right\}=A_{2} e^{\mu \delta_{k}}+B_{2} e^{-\psi \delta_{k}} \\
& E\{\Delta\}=C_{2} e^{\mu R s}+D_{2} e^{-\mu R S}
\end{aligned}
$$

Substituting equation (4-113) into equation (4-95), noting the equality of $\delta_{k}$ and $x_{k}$ by equations (4-88a) and (4-88b), and simplifying,

$$
E\{\Delta\}\left(A_{2} e^{\mu x_{k}}+B_{2} e^{-\mu x_{k}}\right)=\left(A_{2} e^{\mu x_{k}}+B_{2} e^{-\mu x_{k}}\right)\left(e^{\mu R s}+e^{-\mu R s}\right)
$$

Cancelling common terms shows that 


$$
E\{\Delta\}=e^{\mu R s}+e^{-\mu R \Delta}
$$

Thus $C_{2}=D_{2}=1$ in equation (4-114). Substituting the boundary conditions for $\Psi$,

$$
\Psi\{0\}=k_{\min }=\frac{K_{\max }^{*}}{2 R^{2}}=A_{2}+B_{2}
$$

and $\psi\left(\left\{\delta_{\text {mox }}\right\}=k_{\max }=\frac{K_{\max }^{\bullet}}{2 R^{2}}=A_{2} e^{\mu \delta_{m o x}}+B_{2} e^{-\mu \omega_{m x x}}\right.$

Solving for the unknown coefficients,

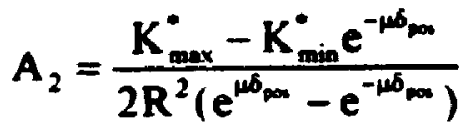

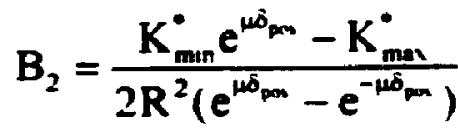

Equation (4-113) then becomes (for a general displacement),

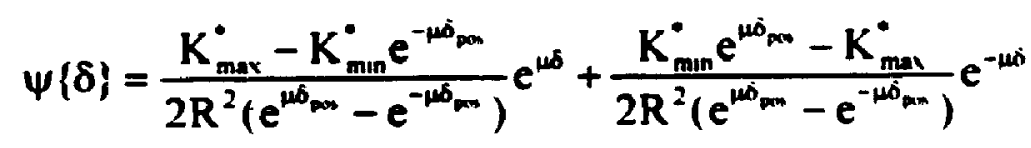

However, exponentials can also be represented in hyperbolic form. The hyperbolic definitions are

$$
\begin{aligned}
& \sinh u=\frac{1}{2}\left(e^{u}-e^{-u}\right) \\
& \cosh u=\frac{1}{2}\left(e^{u}+e^{-u}\right)
\end{aligned}
$$

Rearranging equation (4-116) and replacing exponentials with their hyperbolic counterparts,

$$
\Psi\{\delta\}=\frac{K_{\max }^{*} \sinh (\mu \delta)+K_{\min }^{*} \sinh \left(\mu\left(\delta_{p o s}-\delta\right)\right)}{2 R^{2} \sinh \left(\mu \delta_{p o s}\right)}
$$

The only boundary condition on $E\{\Delta\}$ is from $\xi\{0\}=0$, or $E\{0\}=2$ by equation (4.94). Substituting this condition into equation (4-115),

$$
E\{\Delta\}=e^{0}+e^{0}=2
$$

This means that the boundary condition is met identically for any value of $\mu$. In order to solve for $\mu$, another boundary condition must be created. This is freely chosen in the 
form $\xi\left\{\Delta_{0}\right\}=\xi_{0}$. This means that an equation can be found given the conditions that the joint stiffness changes by $\xi_{0}$ for an endpoint deflection of $\Delta_{0}$. Substituting $\xi\{\Delta\}$ for $E\{\Delta\}$ by equation (4-94), the hyperbolic cosine, and the above boundary conditions into equation (4-115), and rearranging,

$$
\mu=\frac{\cosh ^{-1}\left(\xi_{0}+1\right)}{R \Delta_{0}}
$$

Substituting equation (4-118) into equation (4-117), the spring stiffness becomes

$$
\Psi\{\delta\}=\frac{K_{\max }^{*} \sinh \left(\frac{\cosh ^{-1}\left(\xi_{0}+1\right)}{R \Delta_{0}} \delta\right)+K_{\operatorname{mun}}^{*} \sinh \left(\frac{\cosh ^{-1}\left(\xi_{0}+1\right)}{R \Delta_{0}}\left(\delta_{p o s}-\delta\right)\right.}{2 R^{2} \sinh \left(\frac{\cosh ^{-1}\left(\xi_{0}+1\right)}{R \Delta_{0}} \delta_{\text {pos }}\right)}
$$

Note that there is no solution for $\mu$ in equation (4-118) for $\xi_{0}<0$. Thus equation (4-119) only applies for $\xi>0$. Errors greater than 0 mean that the joint stiffness is increasing as it is deflected. This is often referred to as a hardening spring.

It is important to understand what equation (4-119) represents. The original conditions state that $\xi=\xi\{\Delta\}$, meaning that it is independent of joint stiffness. This means that the joint will increase by the same percentage for the same joint deflection at any stif hess. For example, if $\xi_{0}=1$ (100\% increase) at $\Delta_{0}=\pi / 2$ radians (90 degrees), the joint stiffness will double for any set stiffness when it is deflected by 90 degrees.

Case 3: $\lambda<0$

To force $\lambda$ negative, let $\lambda=-\mu^{2}$. The solutions are then of the form

$$
\begin{aligned}
& \Psi\left\{\delta_{k}\right\}=A_{3} \cos \left(\mu \delta_{k}\right)+B_{3} \sin \left(\mu \delta_{k}\right) \\
& E\{\Delta\}=C_{3} \cos (\mu R \Delta)+D_{3} \sin (\mu R \Delta)
\end{aligned}
$$

Substituting equation (4-120) into equation (4-95) and simplifying, 


$$
E(\Delta\}\left(A_{3} \cos \left(\mu \delta_{k}\right)+B_{3} \sin \left(\mu \delta_{k}\right)\right)=2\left(A_{3} \cos \left(\mu \delta_{k}\right)+B_{3} \sin \left(\mu \delta_{k}\right)\right) \cdot \cos (\mu R \Delta)
$$

Therefore,

$$
E\{\Delta\}=2 \cos (\mu R \Delta)
$$

Thus $C_{3}=2$ and $D_{3}=0$ in equation (4-121). Substituting the stiffness boundary conditions into equation (4-120),

$$
\psi\{0\}=\frac{K_{\text {map }}^{\cdot}}{2 R^{2}}=A_{3} \cos (0)+B_{3} \sin (0)=A_{3}
$$

and $\quad \psi\left(\delta_{p m}\right\}=\frac{K_{m a x}^{\bullet}}{2 R^{2}}=\frac{K_{m i n}^{\bullet}}{2 R^{2}} \cos \left(\mu \delta_{p o s}\right)+B_{3} \sin \left(\mu \delta_{p m s}\right)$

Rearranging,

$$
B_{3}=\frac{K_{\max }^{\cdot}-K_{\min }^{\bullet} \cos \left(\mu \delta_{p o s}\right)}{2 R^{2} \sin \left(\mu \delta_{p o s}\right)}
$$

Equation (4-120) then becomes (for a general displacement)

$$
\psi\{0\}=\frac{K_{\max }^{*} \sin (\mu \delta)+K_{\min }^{*} \sin \left(\mu\left(\delta_{p o x}-\delta\right)\right)}{2 R^{2} \sin \left(\mu \delta_{p o s}\right)}
$$

Substituting the boundary condition $E\{0\}=2$ into equation (4-122),

$$
E\{0\}=2 \cos (0)=2
$$

Again the boundary is met identically for any value of $\mu$. Substituting the boundary condition from Case 2 and $\xi\{\Delta\}$ from equation (4-94) in equation (4-122) and rearranging,

$$
\mu=\frac{\cos ^{-1}\left(\xi_{0}+1\right)}{R \Delta_{0}}
$$

Substituting equation (4-124) into equation (4-123), the spring stiffness function is 


$$
\psi\{\delta\}=\frac{K_{\max }^{\cdot} \sin \left(\frac{\cos ^{-1}\left(\xi_{0}+1\right)}{R \Delta_{0}} \delta\right)+K_{\text {min }}^{\cdot} \sin \left(\frac{\cos ^{-1}\left(\xi_{0}+1\right)}{R \Delta_{0}}\left(\delta_{p o s}-\delta\right)\right.}{2 R^{2} \sin \left(\frac{\cos ^{-1}\left(\xi_{0}+1\right)}{R \Delta_{0}} \delta_{p o s}\right)}
$$

Note that there is no solution for $\mu$ in equation (4-124) for $\xi_{0}>0$. Thus equation (4-125) only applies for $\xi<0$. In this case, the error is negative so the joint stiffness decreases as it is deflected. This is referred to as a softening spring. Also note the similarity between equations (4-119) and (4-125). The only difference is the use of hyperbolic trigonometric functions in equation (4-119) while equation (4-125) contains circular trigonometric functions.

Spring stiffness functions that cause a decrease in joint stiffness for endpoint deflections (hardening joint spring) are represented by equation (4-119). Spring stiffness functions that cause an increase in joint stiffness for endpoint deflections (softening joint spring) are represented by equation (4-125). Spring stiffness functions that cause no change in joint stiffness for endpoint deflection, i.e., stiffness is independent of deflection, are represented by equation (4-112). This was also discovered earlier in equation (4-39).

As previously mentioned, equations (4-112), (4-119), and (4-125) correspond to spring functions for which the percentile change in joint stiffness for a given deflection is independent of the joint stiffness. Any spring function that is not in one of these forms results in a changing joint stiffness that depends on t'-a set stiffness. While such a case is more general than the equations developed here, there are an infinite number of possible spring function forms. Therefore such an analysis cannot show a clear relationship between spring function and joint stiffness error. 
The stiffness functions of equations (4-119) and (4-125) are plotted in Figure 4.12 for $\xi_{0}= \pm 0.5(5 \%)$ and $\xi_{0}= \pm 1(100 \%)$ at $\Delta_{0}=\pi / 2$ radians (90 degrees) endpoint deflection. This means that if the endpoint is deflected 90 degrees, the joint stiffness will increase (or decrease) by $5 \%$ and $100 \%$. Note from the graph that with these larger changes in stiffiness, the individual spring stiffness functions do not differ far from the linear case. It is also interesting to note that in both cases the individual springs both increase in stiffness with deflection, i.e., they are hardening springs. This is true even in the case where they combine at the joint to form a softening spring.

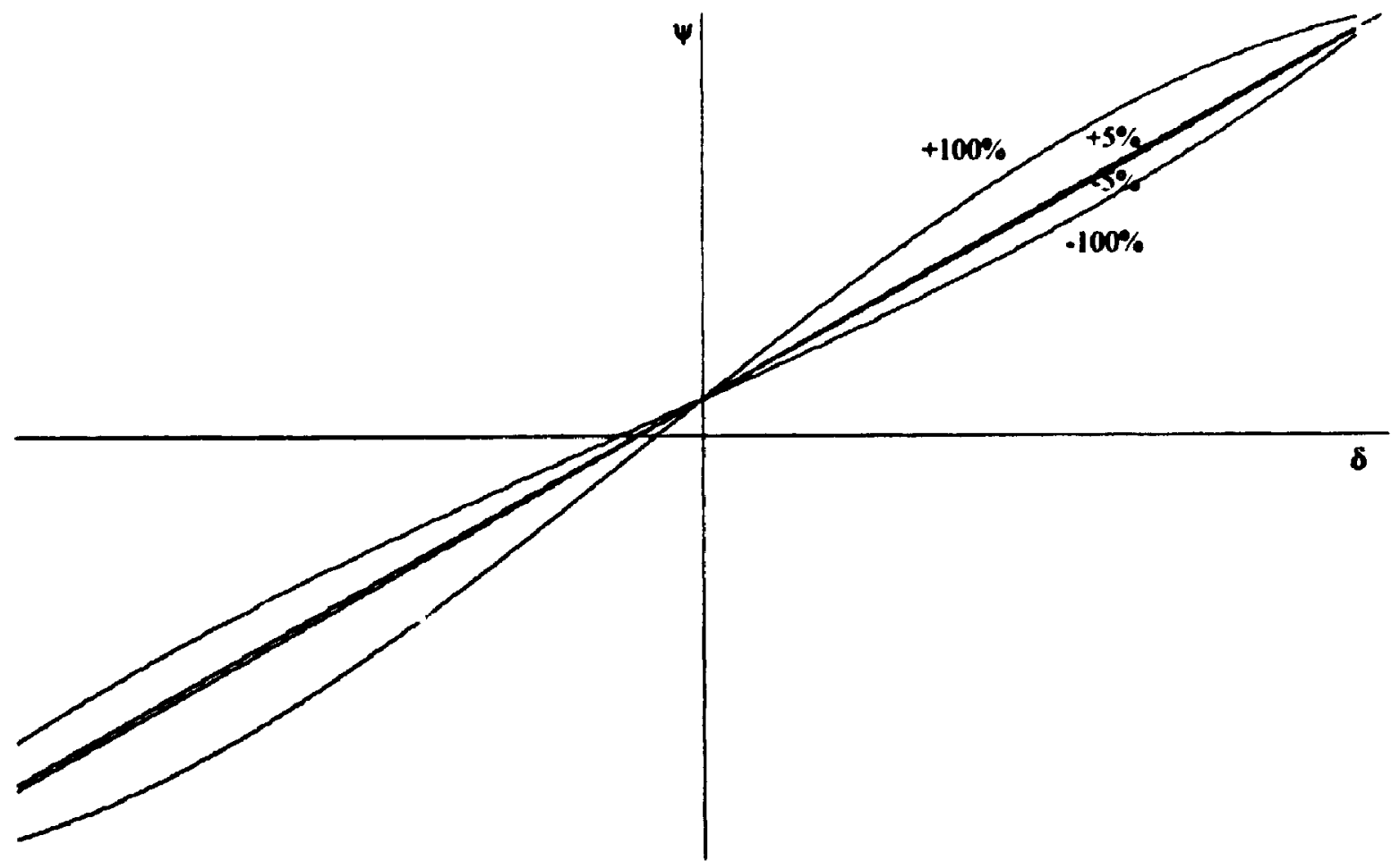

Figure 4.12. Spring stiffness functions that change joint stiffness by $\pm 5 \%$ and $\pm 100 \%$ for a 90 degree endpoint deflection 


\subsection{General Limitations}

If the system is designed such that the joint stiffness and endpoint deflection are not independent, as in the previous section, the limitations presented in Section 4.2 are not completely valid. The geometric analysis, up to equation (4-72), remains identical for any spring functions since no specific functions are introduced. However, the stiffness substitutions and torque limitations are specific for quadratic springs. The geometric limitation for positive endpoint deflections, given by equation (4-72), is

$$
\Delta_{\mathrm{lmm}}=\frac{1}{R}\left[\frac{1}{2} \delta_{\text {pos }}(1+\eta)-\left|\mathrm{x}_{\mathrm{k}}-\frac{1}{2} \delta_{\text {pos }}(1-\eta)\right|\right]
$$

Since $x_{\mathbf{k}}$ is an indication of joint stiffness (it is the controlling variable), there is a relationship between stiffness and the limit of endpoint deflection. This is clear from the fact that if the springs are fully stretched at the same time by $x_{k}=\delta_{p o s}$, then due to the spring 2 displacement in equation (3-7b) there can be no endpoint deflection. The absolute value in the limit indicates that there are two possible solutions: one of the springs becomes stretched to the upper maximum, or one is displaced to its lower limit. Both cases are presented below.

\section{Case 1: Negative Value}

In order for the term within the absolute value to be negative, the condition is

$$
x_{k}-\frac{1}{2} \delta_{\text {pos }}(1-\eta) \leq 0 \text { or } \quad x_{k} \leq \frac{1}{2} \delta_{\text {pos }}(1-\eta)
$$

The limitation of equation (4-72) becomes

$$
\Delta_{\mathrm{lmm}}=\frac{\mathbf{x}_{\mathrm{h}}+\eta \delta_{\mathrm{pos}}}{\mathrm{R}}
$$

Note that this limit simply restates equation (4-70a), recognizing that equation (4-72) is the combination of equations (4-70a) and (4-70b). This limit suggests that large $\eta$ is best. 
As $\eta$ approaches 0 (no negative spring deflection allowed), the joint deflection limit becomes

$$
\Delta_{\mathrm{lum}}=\frac{\mathbf{x}_{\mathbf{k}}}{\mathbf{R}}
$$

Since low values of $x_{t}$ correspond to low joint stiffness, the maximum deflection becomes negligible at low stiffness. This conflicts with the purpose of using low stiffness, namely to allow large deflections at low torques.

Substituting equation (4-126), (4-66), and the equivalence of $x_{1}$ and $\delta_{1}$ from equation (4-88a) into equations (3-7a) and (3-7b), the spring deflections become

$$
\delta_{1}=-\delta_{\text {neg }}
$$

and $\delta_{2}=2 \delta_{k}+\delta_{\text {neq }}$

It can be seen that this case corresponds to spring 1 reaching its negative displacement limit. Substituting these spring deflections into equation (3-10), the torque that pushes the arm to $\Delta_{\mathrm{lum}}$ is

$$
T_{\mathrm{lm}}=R\left(\phi\left\{2 \delta_{\mathrm{k}}+\delta_{\mathrm{neq}}\right\}-\phi\left\{-\delta_{\text {neg }}\right\}\right)
$$

The stiffness elements used in most applications of antagonistic actuation apply the traditional force-displacement spring characteristic of tensile force for positive displacement and compressive force for negative displacement (see Figure 4.13), as well as being monotonic (force increases with deflection). In this arrangement, the force for $\delta=-\delta_{\text {neg }}$ is at a minimum (negative maximum). Thus the minimum force can be defined $F_{\text {min }}=\phi\left\{-\delta_{\text {neg }}\right\}$

Substituting this force into equation (4-127), the limiting torque is

$$
T_{h m}=R\left(\phi\left\{2 \delta_{k}+\delta_{m e g}\right\}-F_{m i n}\right)
$$

As in Section 4.2, the equations are derived for a positive endpoint deflection. A negartve deflection follows a similar derivation with springs 1 and 2 switched due to the symmetry. The resulting limiting torque for a negative endpoint deflection is therefore 


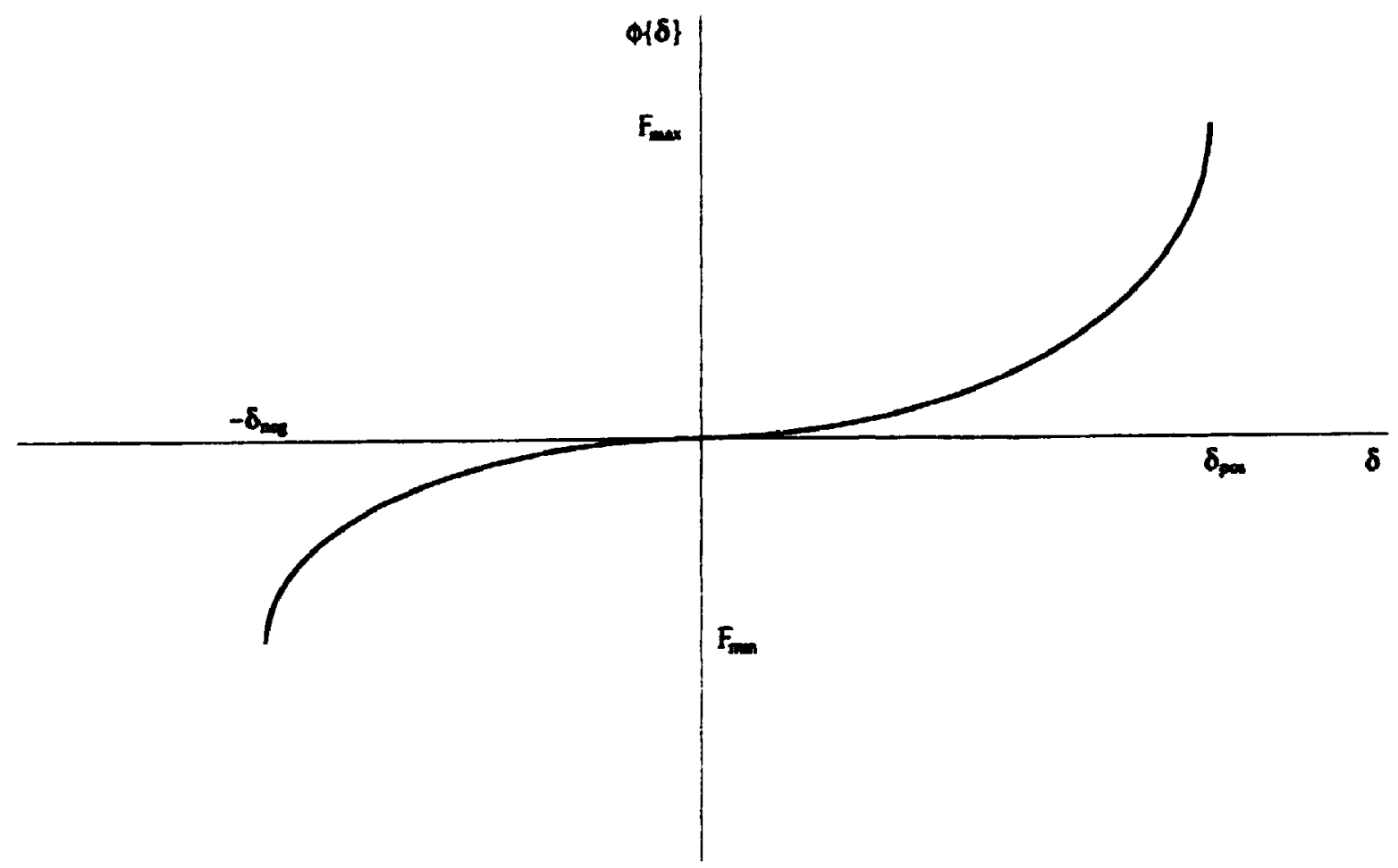

Figure 413 Typical stiffness element force-deflection curve

$$
\mathrm{T}_{\mathrm{ltm}}=R\left(\mathrm{~F}_{\mathrm{mn}}-\phi\left\{2 \delta_{\mathrm{k}}+\delta_{\mathrm{neg}}\right\}\right)
$$

As with the quadratic springs in Section 4.2.2, the torque limit shows the symmetry of the system since the negative torque limit in equation (4-129) is the negative of equation (4128).

\section{Case 2: Positive Value}

The conditions on equation (4-72) for the absolute value term to be positive is

$$
x_{k}-\frac{1}{2} \delta_{\text {pos }}(1-\eta) \geq 0 \text { or } \quad x_{k} \geq \frac{1}{2} \delta_{\text {pos }}(1-\eta)
$$

Note from the conditions that for $0 \leq \eta \leq 1$ this case applies for at least the upper half to full operating range of $x_{k}$, since $0 \leq x_{k} \leq \delta_{\text {pos. }}$. For this case, equation (4-72) becomes

$$
\Delta_{\mathrm{lim}}=\frac{\delta_{\text {pos }}-\mathbf{x}_{\mathrm{h}}}{R}
$$


which is simply a restatement of equation (4-70b). In this case, the deflection limit is larger at low values of $x_{k}$, i.e., low joint stiffness. This corresponds to the desired deflection properties. Substituting equation (4-130) into equations (3-7a) and (3-7b) and using $\delta_{1}$ for $x_{1}$, the individual spring displacements for a positive endpoint deflection are $\delta_{1}=\mathbf{2} \delta_{k}-\delta_{\text {pos }}$

and $\delta_{2}=\delta_{\text {pus }}$

Therefore this case corresponds to spring 2 reaching its maximum positive displacement for a positive endpoint deflection. Substituting these spring displacements into equation (3-10), the torque that pushes the arm to $\Delta_{\mathrm{lm}}$ is

$$
T_{h m}=R\left(\phi\left\{\delta_{\text {pos }}\right\}-\phi\left\{2 \delta_{1}-\delta_{\text {pos }}\right\}\right)
$$

By Figure 4.13 the maximum force is defined as

$$
F_{\text {max }}=\phi\left\{\delta_{\text {pos }}\right\}
$$

Substituting this force into equation (4-131), the limiting torque for posittve endpoint deflection becomes

$$
\mathrm{T}_{\mathrm{hlm}}=\mathrm{R}\left(\mathrm{F}_{\max }-\phi\left\{2 \delta_{\mathrm{k}}-\delta_{\mathrm{pos}}\right\}\right)
$$

The corresponding torque for a negalive endpoint deflection, with the springs reversed, is

$$
\mathrm{T}_{\mathrm{ltm}}=R\left(\phi\left\{2 \delta_{\mathrm{k}}-\delta_{\text {pos }}\right\}-\mathrm{F}_{\max }\right)
$$

Looking at the limiting torques, i.e., equations (4-128), (4-129), (4-132), and (4-133), suggests that the maximum positive torque that can be achieved within the buunds of variable stiffness is

$$
T_{l \text { IIm }}=R\left(F_{\text {max }}-F_{\text {min }}\right)
$$

By equation (4-43), this torque translates to

$$
T_{\text {lim }}=R F_{\text {renpe }}
$$

In hindsight, this is apparent from the configuration of the system. The limiting torque is that for which both springs push with their maximum force, as seen in Figure 4.14. Note 
from Figure 4.13 that $F_{\min }$ is a negative force for most stiffness elements. The corresponding negative torque limit is the reverse case, where spring 2 is at a maximum and spring 1 is at a minimum, and thus is the negative of equation (4-134).

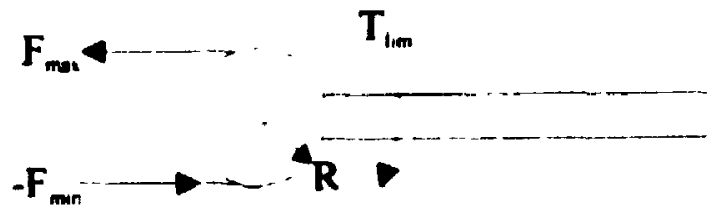

Figure 4 14. Positive limiting torque.

By equation (4-128), this maximum limiting torque is achieved if

$$
2 \delta_{\mathrm{k}}+\delta_{\text {neg }}=\delta_{\text {pos }}
$$

or $\delta_{\mathrm{a}}=\frac{1}{2}\left(\delta_{\mathrm{pms}}-\delta_{\mathrm{meq}}\right)=\frac{1}{2} \delta_{\mathrm{pma}}(1-\eta)$

In other words, for $\eta=0$ this occurs at $\delta_{\mathrm{k}}=1 / 2 \delta_{\text {poss }}$, which corresponds to some joint stiffness between minimum and maximum. For $\eta=1$, the maximum limiting torque occurs at $\delta_{k}=0$, which corresponds with minimum joint stiffness.

By equation (4-133), the maximum limiting torque is achieved if

$$
2 \delta_{\mathrm{A}}-\delta_{\text {pos }}=-\delta_{\text {neq }}=-\eta \delta_{\text {pos }}
$$

or $\delta_{k}=\frac{1}{2} \delta_{\text {pors }}(1-\eta)$

Again, for $\eta=0$ this occurs at $\delta_{k}=1 / 2 \delta_{\text {max }}$. For $\eta=1$, the maximum limiting torque still occurs at $\delta_{k}=0$. The reason for this is because the range of deflection for $\eta=0$ is between $\delta=0$ and $\delta=\delta_{\text {pos }}$, whereas for $\eta=1$ it is between $\delta=\delta_{\text {neg }}$ and $\delta=\delta_{\text {pos }}$. The negative limiting torque follows the same results. Figure 4.15(a) shows the general shape for these results. Note that the shape of the boundaries depends upon the actual spring force function. Figure 4.15(b) shows the resulting torque behavior of the joint. The operating lines for various stiffnesses are shown. They are curved because the joint stiffness tends to increase (or decrease) with deflection for non-quadratic springs, as 


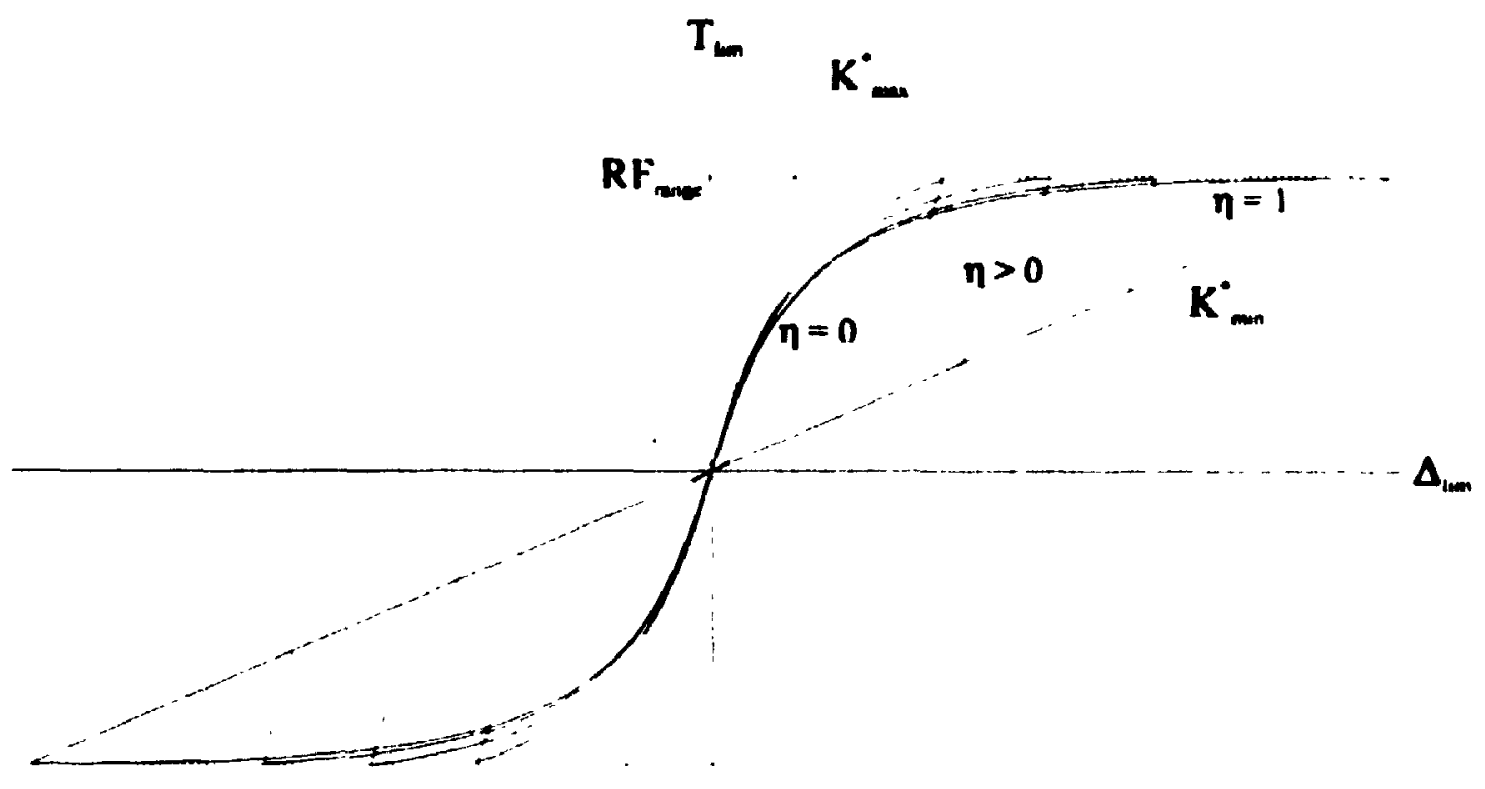

(a)

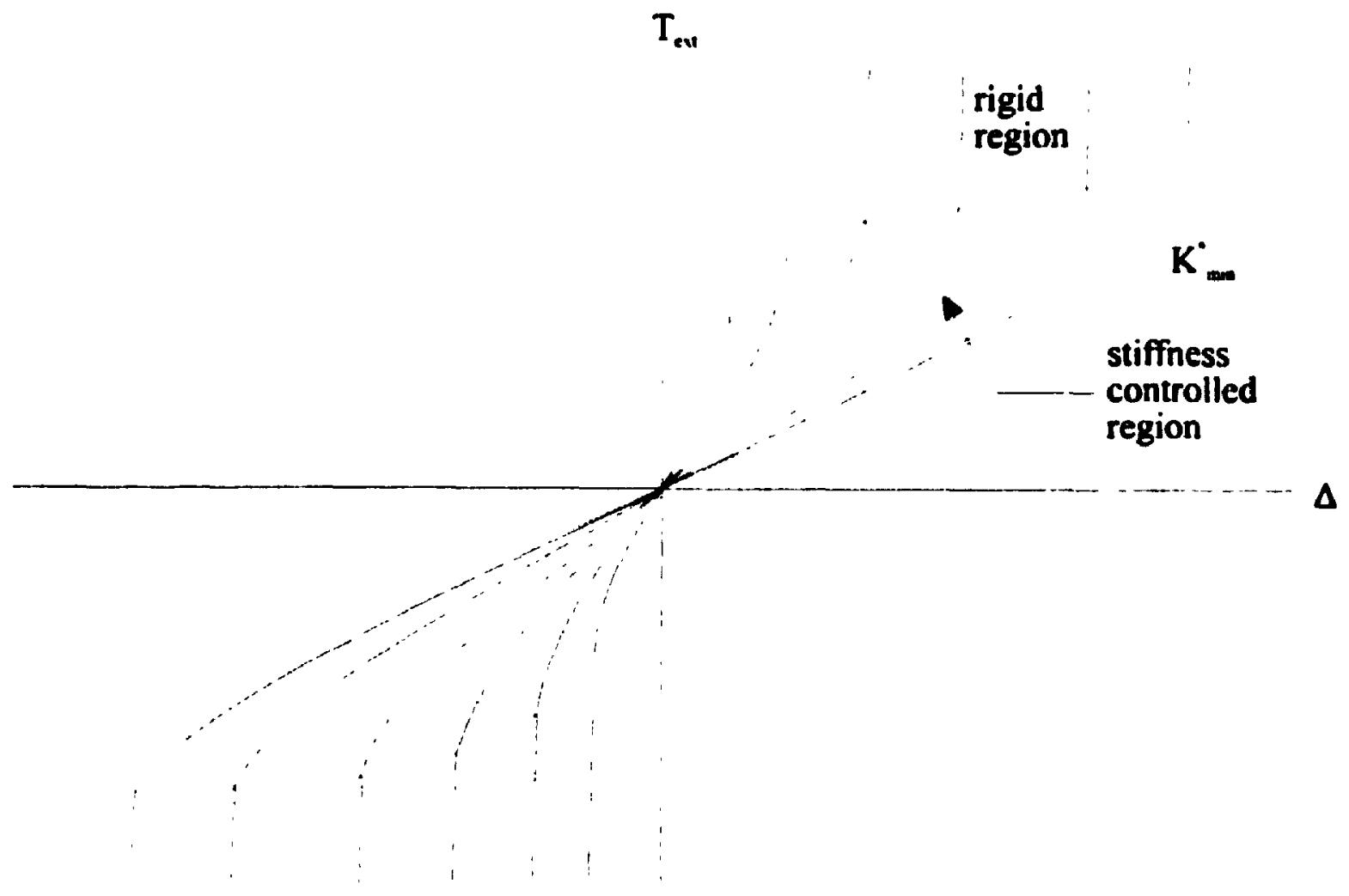

(b)

Figure 4.15: Variable stiffiness limitations (a) general shape of limit region, (b) torque regions. 
shown in the previous section. Within the torque limitations, the arm acts as a variable stiffness joint. As in Section 4.2.2, this area is the stiffness controlled region. Beyond this limit, the arm stiffness is that of the gearing and link materials as in a rigid arm, thus denoted the rigid region.

As with the quadratic springs, the upper limit curve of Figure 4.15(a) corresponds to one of the springs reaching maximum positive deflection, which is the only resulting case for $\eta=1$ (equal stretch and compression limits). The lower limit is $\mathrm{K}_{\min }{ }_{\text {and }}$ ane right-hand limit (left-hand for negative torque) corresponds to the case where one spring reaches maximum compression. Looking at the general upper limit of equation (4-132), the limiting torque is maximum for any given value of $\delta_{k}$ if $\phi\{\delta\}$ remains as small (or negative) as possible. Since it must reach $F_{\max }$ at $\delta_{\text {pos }}$, the best force curve to maximize the stiffness controlled region is one that remains small for most $\delta$ and increases quickly to $F_{\max }$ only near $\delta_{\text {pos }}$, as shown in Figure 4.16. However, it is important to note that if slope of the curve changes very quickly over a short deflection range, it is difficult to properly set the stiffness. The example in Figure 4.16 has a low stiffness (slope of force curve) for most of the springs stretch and changes to high stiffness over a short deflection region. It would require high precision to set the stiffness at some intermediate value. The quadratic stiffness avoids this problem since the stiffness changes linearly with deflection.

The general shape of the force function in Figure 4.16 is compatible with quadratic springs but requires proper selection of the function. To match the shape, the vertex of the quadratic function must occur at the maximum negative displacement. Equation (4-45) indicates the location of the vertex for the function.

$$
\delta=-\frac{K_{\min }^{*}}{K_{\max }^{*}-K_{\min }^{*}} \cdot \delta_{\operatorname{mos}}
$$

Substituting $-\delta_{\mathrm{mg}}$ for $\delta$ and rearranging, the minimum stiffness must be 


$$
K_{\operatorname{mpn}}^{*}=\frac{\eta}{1+\eta} \cdot K_{\max }^{*}
$$

Selection of $\eta<1$ cuts off the end of the stiffness control region, which is not desirable. For $\eta=1$, this means that minimum stiffness must be half of maximum stiffness.

Certainly this is an unacceptable requirement. Therefore some sacrifice must be made. If quadratic springs are to be used, the resulting stiffness controlled region will not be able to handle maximum torque at minimum stiffness, but at some intermediate value as shown in Figure 4.11(b). The alternative is to use springs with the force shape of Figure 4.16 that do not comply with the quadratic form of equation (4-50). However, the resulting operation will not decouple joint stiffness from endpoint deflection. This means that there is a tradeoff between desired operation and performance.

Since most stiffness elements do not typically compress as far as they stretch, it can be assumed that $\eta$, in general, is some intermediate value between 0 and 1 . The lower limit is then defined by equation (4-128). Since it is part of the lower limit, it is best to keep it as small as possible. Again, this implies that $\phi\{\delta\}$ should remain as small as possible. Limits for which $\Phi\{\delta\}$ increases quickly mean that the lower limit also increases quickly. Following these arguments, the stiffness controlled region that corresponds to Figure 4.16 are shown in Figure 4.17.

Application of these spring functions reveals an apparent pattern between the shape of the spring force function and the resulting stiffness controlled region. In general, to get the upper torque limit, the force curve shape of the individual springs is inverted both horizontally and vertically (or rotated 180 degrees), multiplied by the joint radius, and shifted to pass through the origin. The lower limit is the same shape and orientation as the stiffness function, multiplied by the joint radius, and shifted in position. Note that it is the range of force of the springs that is important, and not the absolute force values. Thus the location of the shape on the force-deflection curve is irrelevant to the stiffness control region, but does affect intemal forces. This pattern is graphically 
apparent from the above figure as well as Figure 4.10 for the quadratic springs. The limiting torque equations, i.e., equations (4-128), (4-129), (4-132), and (4-133), prove these apparent graphical relationships. For a positive torque, the upper limi+ is defined by equation (4-132),

$$
T_{h m}=R\left(F_{\max }-\phi\left\{2 \delta_{k}-\delta_{\text {pos }}\right\}\right)
$$

The function is mirrored horizontally by the fact that $\Delta$ varies negatively with $\delta_{1}$ (by equation (3-7a)) (note that the term within the braces is $\delta_{1}$ ), and hence the transformation from the $\delta$ axis to the $\Delta$ axis reverses the direction. The function is mirrored vertically by the negative sign before $\phi$. The inclusion of $\delta_{p o s}$ and $F_{\max }$ shift the function to the origin, and $\mathrm{R}$ converts the force function to a torque. The lower limit for positive deflection is defined by equation (4-128),

$$
T_{h m}=R\left(\phi\left\{2 \delta_{k}+\delta_{\text {neg }}\right\}-F_{m i n}\right)
$$

In this case, $\delta_{1}$ has reached its negative limit and the limiting torque is dependent on $\delta_{2}$. Since $\Delta$ varies in the same direction as $\delta_{2}$ (by equation (3-7b)), there is no horizontal reflection. As well, there is no negative sign in front of $\phi$ so the function is not mirrored vertically. However, $\delta_{\text {neq }}$ and $F_{\min }$ do shift the curve to the origin and $R$ converts the force

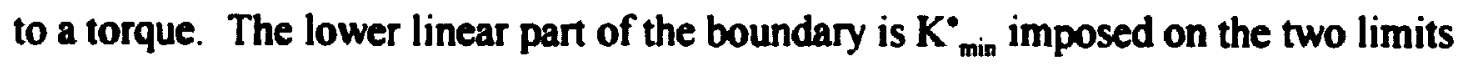
above since the joint stiffness is not designed to operate below $\mathrm{K}_{\text {min }}$. Similar analysis of equations (4-129) and (4-133) gives the same results for the negative torque case. This can also be inferred by symmetry. As an example of the relation between force function and limiting torque, the force-deflection curve of Figure 4.13 results in a stiffness controlled region approximated by Figure 4.18 , using only graphical interpretation. 


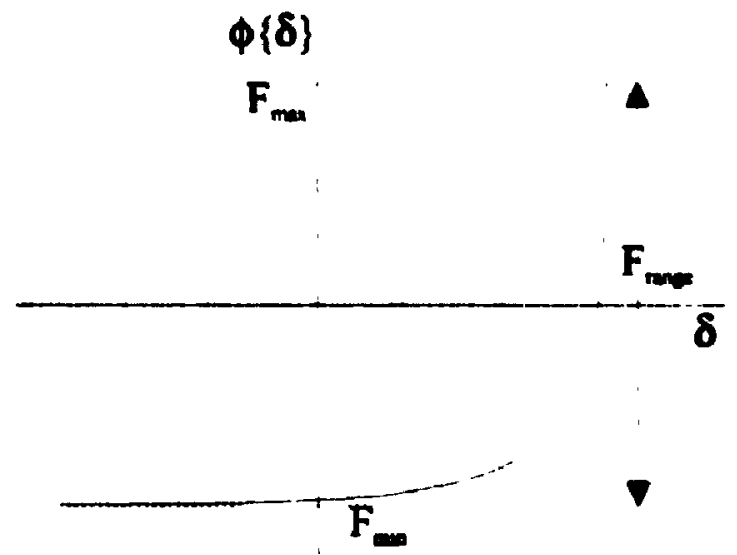

Figure 4.16: Spring force function that results in a large stiffness control region

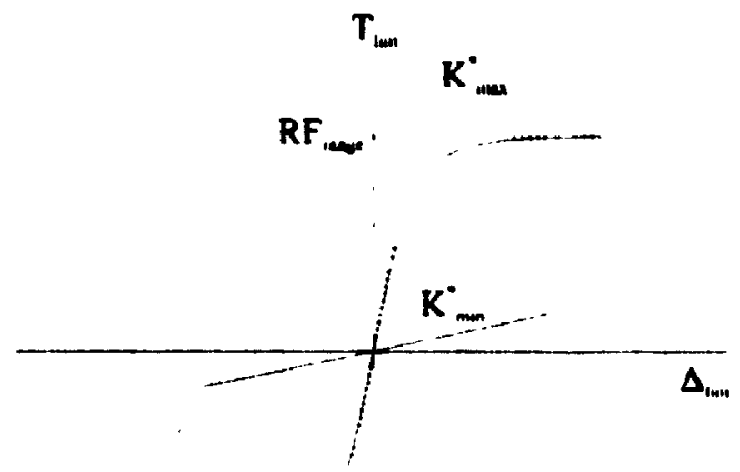

Figure 4.17: Corresponding stiffness controlled region for Figure 416

$T_{\text {tun }}$

$$
\mathbf{K}^{*}
$$

$\mathbf{R F}_{\text {rems }}$

1

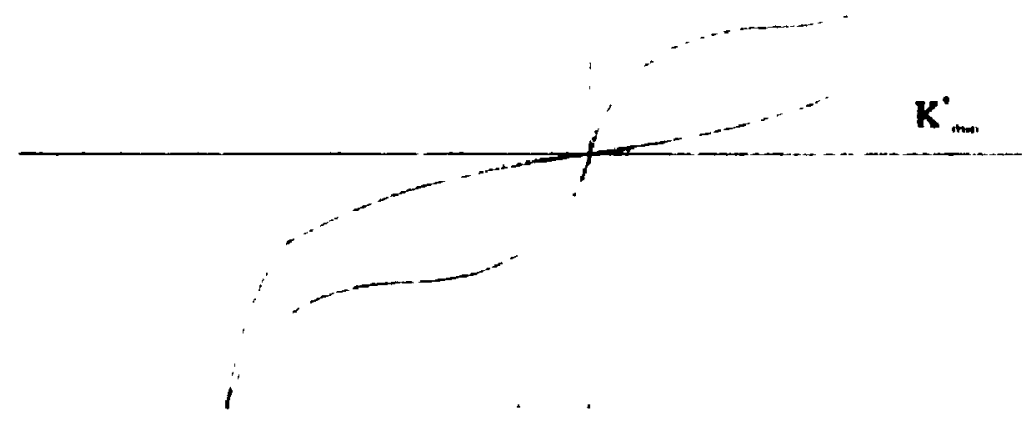

$\Delta_{\text {um }}$

Figure 4.18: Approximate stifiness controlled region using stiffiness elements defined by Figure 413 
Following the relationship between force function and stiffness controlled region, as $\phi$ approaches a linear force curve, the stiffness controlled region approaches a straight line. If $\phi$ is chosen s!uch that the slope decreases, i.e., a softening spring, the roles of the limiting equations switch. Equation (4-128) becomes the upper limit and (4-132) becomes the lower limit. However, softening springs means that $\mathrm{K}^{*}{ }_{\max }$ is associated with no deflection of the springs and $\mathrm{K}_{\operatorname{mn}}^{*}$ is associated with maximum stretch. But the compression region of the spring was necessary so that joint deflection was allowed at minimum stiffness. For softening springs this region allows large deflections at maximum stiffness and the joint is effectively rigid at minimum stiffness. Thus the geometry of the springs must be changed for softening springs. so no clear performance conclusions can be made from the relationships developed above.

\subsection{Power and Energy Requirements}

Power is defined as the change of energy with time. By the definition of $x_{k}$ and $\mathbf{x}_{\text {mnc }}$ (Table 3B), it is apparent that no power should ideally be required to perform motion in an unloaded situation. Loading changes the energy state of the springs. When an endpoint deflection occurs due to loading, the length of each spring changes. This changes the stored energy. Since energy cannot be created, the net static increase of energy in the springs must be equal to that supplied externally. Dynamically, external energy is also dissipated in the dampers and storcd in the inertia of the arm. However, these types of energy changes are a function of the motion and not of the springs. Thus they do not affect the spring design. 


\subsubsection{Motion Energy}

Figures 4.6 and 4.7 introduce two configurations for the implementation of this system. In the first configuration, the performance of a motion without a stiffness change requires one of the branch actuators to pull against a force a specified distance. Thus the actuator does work and requires energy to do so. The second actuator moves the same distance in the same direction as the force. Thus an identical amount of work is done on the second actuator. However, this energy is not retrievable unless the actuator can act as a generator. Therefore, unloaded motion for Configuration 1 (Figure 4.6) requires the expenditure of energy.

The same phenomenon does not apply to Configuration 2 (Figure 4.7). The performance of an unloaded motion requires that the position actuator rotate to a new position. Since there is no net torque on the position actuator, there is no energy required to perform the motion, neglecting losses within the actuator and assuming no inertial mass. This configuration takes advantage of the fact that the branch forces are equal and opposite. By connecting them around a radius, the net torque is zero. Note, however, that the branch forces are equal only when the joint is unloaded. In a loaded situation, the external torque is equal to the difference in branch torques (force multiplied by joint radius), by equation (3-8). Since these are the same forces that act on the motion actuator, the net torque is equivalent to the external torque. The energy required to perform a loaded motion is therefore the same as the work performed externally, once again neglecting losses is the dampers. 


\subsubsection{Stiffness Energy and Power}

By definition in Table 3B, a change in $x_{k}$ causes a change in the energy stored in the springs. This implies that an input of energy is required to increase stiffness, and energy is released when stiffness is decreased. Looking at Configurations 1 and 2 (Figures 4.6 and 4.7), it can be seen that in both cases an increase in stiffness requires work to be performed on the springs. For a decrease in stiffness, work is performed by the springs on the actuator(s), which cannot be recovered unless the actuator(s) can operate as a generator. Mechanical energy is defined as a force through a distance. Thus the instantaneous energy in either spring is defined as

$$
d E_{1,2}=\phi_{1,2}\left\{\delta_{1,2}\right\} \cdot d \delta_{1,2}
$$

where $E_{1}$ and $E_{2}$ are the energy stored in springs 1 and 2, respectively. The total energy stored in each of the springs is the instantaneous energy summed (integrated) over the change in stretch. Integrating equation (4-135), the energy in a spring is given by

$$
E_{L, 2}=\int_{\delta_{\Lambda}}^{\delta_{B}} \phi_{L, 2} \cdot d \delta_{1,2}
$$

Power is defined as the change in energy over time. The power requirement to stretch the springs is therefore

$$
P_{1.2}=\frac{d_{1.2}}{d t}
$$

where $P_{1}$ and $P_{2}$ are the power to stretch springs 1 and 2 , respectively. Note that additional power is also required to change the stiffness to overcome the force within the dampers. However, this does not affect the power requirements of the springs.

Substituting the spring energy from equation (4-136) into equation (4-137) and using the chain rule, the spring power becomes

$$
P_{1,2}=\frac{\partial E_{L, 2}}{\partial \delta_{1,2}} \cdot \frac{d \delta_{L 2}}{d t}=\frac{\partial}{\partial \delta}\left(\int_{\delta_{A}}^{\delta_{1.2}} \phi_{1,2} \cdot d \delta_{L, 2}\right) \cdot \dot{\delta}_{L, 2}=\phi \cdot \dot{\delta}_{L, 2}
$$


where

$$
\dot{\delta}_{1,2}=\frac{d \delta_{12}}{d t}
$$

Equation (4-139) is the rate at which the spring is stretched. This is independent of the spring force function. By equation (4-138), the power requirement is directly proportional to the force function of the spring. A spring whose force remains near zero would require the least power. Such a force-deflection function is shown in Figure 4.19(a). It is interesting to note that the shape of the positive half of this curve corresponds to the shape that maximizes the stiffness controlled region from Section 4.4. Combining the minimum power and maximum stiffness controlled region criteria, the best curve shape, for a given force range, is shown in Figure 4.19(b). However, given a maximum and minimum force that the stiffness element can produce, larger limiting torques can be developed by the curve of $4.19(\mathrm{a})$. It is likely that an optimized curve lies somewhere between these two curves, depending on the importance of the two criteria.
$\phi\{\delta\}$
$\phi\{\delta\}$
$\mathbf{F}_{\max }$
$\mathbf{F}_{\max }$

$\mathbf{F}_{\mathrm{mon}}$

(a)

(b)

Figure 4.19: Ideal force curves for minimizing power, (a) for given force range, (b) combined with maximum stiffness controlled region criterion. 


\subsubsection{Power: Relation to Joint Stiffness Error}

The effect of choosing non-quadratic stiffness elements on joint stiffness changes due to endpoint deflections is shown in Section 4.3. Using the relationships developed in that section, a correlation between power reduction and joint stiffness error can be found. Since the power required to stretch each spring is directly proportional to the spring force, a power savings index for each spring, given an arbitrary force function different from quadratic, can be defined as

$$
p=\frac{\phi_{q}-\phi_{e}}{\phi_{q}}=1-\frac{\phi_{c}}{\phi_{q}}
$$

where $p=$ normalized power savings in relation to that of quadratic springs

$\phi_{\mathrm{q}}=$ quadratic force function (equation (4-50))

$\phi_{e}=$ non-quadratic force function

Equations (4-1 19) and (4-125) express the relationship between joint stiffness and endpoint deflection in the form of spring stiffnesses. To evaluate their relationship to power savings via equation (4-140), they must first be integrated into force form.

Equation (4-119) represents the spring stiffness relation that results in increasing joint stiffness for positive endpoint deflections. It is therefore denoted at $\psi_{\epsilon}$. where

$$
\Psi_{e+}\{\delta\}=\frac{K_{\max }^{*} \sinh \left(\frac{\cosh ^{-1}\left(\xi_{0}+1\right)}{R \Delta_{0}} \delta\right)+K_{\min }^{\cdot} \sinh \left(\frac{\cosh ^{-1}\left(\xi_{0}+1\right)}{R \Delta_{0}}\left(\delta_{\max }-\delta\right)\right)}{2 R^{2} \sinh \left(\frac{\cosh ^{-1}\left(\xi_{0}+1\right)}{R \Delta_{0}} \delta_{\max }\right)}
$$

Integrating with respect to $\delta$, the "positive error" force function is

$$
\phi_{e+}\{\delta\}=\frac{K_{\max }^{*} \cosh (\mu \delta)-K_{\min }^{*} \cosh \left(\mu\left(\delta_{\max }-\delta\right)\right)}{2 \mu R^{2} \sinh \left(\mu \delta_{\max }\right)}+C_{e+}
$$


where $C_{e}$ is the constant of integration, and from equation (4-118),

$$
\mu=\frac{\cosh ^{-1}\left(\xi_{0}+1\right)}{R \Delta_{0}}
$$

Similarly, equation (4-125) represents the spring stiffness relation that results in decreasing joint stiffness for positive endpoint deflections. It is therefore denoted at $\Psi_{\text {r }}$ where

$$
\Psi_{e-}\{\delta\}=\frac{K_{\max }^{*} \sin \left(\frac{\cos ^{-1}\left(\xi_{0}+1\right)}{R \Delta_{0}} \delta\right)+K_{\min }^{\cdot} \sin \left(\frac{\cos ^{-1}\left(\xi_{0}+1\right)}{R \Delta_{0}}\left(\delta_{\max }-\delta\right)\right)}{2 R^{2} \sin \left(\frac{\cos ^{-1}\left(\xi_{0}+1\right)}{R \Delta_{0}} \delta_{\max }\right)}
$$

Integrating with respect to $\delta$, the "negative error" force function is

$$
\phi_{e-}\{\delta\}=\frac{-K_{\max }^{\cdot} \cos (\mu \delta)+K_{\min }^{\cdot} \cos \left(\mu\left(\delta_{\max }-\delta\right)\right)}{2 \mu R^{2} \sinh \left(\mu \delta_{\max }\right)}+C_{e-}
$$

where $C_{e}$ is the constant of integration, and from equation (4-124),

$$
\mu=\frac{\cos ^{-1}\left(\xi_{0}+1\right)}{R_{e} \Delta_{0}}
$$

Selecting integration constants requires an understanding of the reason for determining the force functions. The purpose of these integrations is to compare force functions by equation (4-140) to determine power savings over quadratic springs. Since power is proportional to force by equation (4-138), no power is required at the point where the spring force is zero. Therefore no power savings can be achieved by any other function at the point where the quadratic force function is zero. In fact, any force function that is not also equal to 0 at that deflection requires more power at that point. Thus the constants of integration in equations (4-141) and (4-142) should be chosen so that the forces pass through 0 at the same point as the quadratic force of equation (4-50). Setting equation (4-50) equal to zero and rearranging, the spring deflection that creates zero force is 


$$
\delta=\frac{\delta_{\max }}{K_{\max }^{*}-K_{\min }^{*}}\left(-K_{\min }^{*} \pm \frac{1}{\sqrt{2}} K_{\max }^{\cdot}\right)
$$

The power being examined is for changes in stiffness. The boundary conditions of the stiffness are that it is minimum for $x_{k}=\delta_{k}=0$ and maximum for $x_{k}=\delta_{k}=\delta_{\text {max }}$. Therefore the stiffness is set by positive displacements of the springs. In addition, for minimum stiffnesses greater then zero and $\eta<1$, the quadratic function may not return to zero for negative displacements, as in Figure 4.3. For both of these reasons, the positive value of $\delta$ should be used from equation (4-143). Denoting this "zero force" displacement as $\delta_{0}$, it is given by

$$
\delta_{0}=\frac{\frac{1}{\sqrt{2}} \mathbf{K}_{\max }^{*}-\mathbf{K}_{\min }^{\cdot}}{\mathbf{K}_{\max }^{*}-\mathbf{K}_{\min }^{*}} \delta_{\max }
$$

Substituting $\delta_{0}$ into equation (4-141), the integration constant for the "positive error" force function is

$$
C_{e+}=-\frac{\left.K_{\max }^{\cdot} \cosh \left(\mu \delta_{0}\right)-K_{\min }^{*} \cosh \left(\mu ; \delta_{\max }-\delta_{0}\right)\right)}{2 \mu R^{2} \sinh \left(\mu \delta_{\max }\right)}
$$

Introducing this constant into equation (4-141), the force function becomes

$$
\phi_{e+}\{\delta\}=\frac{K_{\max }^{*}\left[\cosh (\mu \delta)-\cosh \left(\mu \delta_{0}\right)\right]-K_{\min }^{*}\left[\cosh \left(\mu\left(\delta_{\max }-\delta\right)\right)-\cosh \left(\mu\left(\delta_{\max }-\delta_{0}\right)\right)\right]}{2 \mu R^{2} \sinh \left(\mu \delta_{\max }\right)}
$$

where $\mu$ is given from equation (4-118) and $\delta_{0}$ is given by equation (4-144). Similarly, substituting $\delta_{0}$ into equation (4-142), the integration constant for the "negative error" force function is

$$
C_{e-}=\frac{K_{\max }^{\cdot} \cos \left(\mu \delta_{0}\right)-K_{\min }^{\cdot} \cos \left(\mu\left(\delta_{\max }-\delta_{0}\right)\right)}{2 \mu R^{2} \sinh \left(\mu \delta_{\max }\right)}
$$

Introducing this constant into equation (4-142), the force function becomes

$$
\phi_{r-}\{\delta\}=\frac{-K_{\max }^{\cdot}\left[\cos (\mu \delta)-\cos \left(\mu \delta_{0}\right)\right]+K_{\min }^{*}\left[\cos \left(\mu\left(\delta_{\max }-\delta\right)\right)-\cos \left(\mu\left(\delta_{\max }-\delta_{0}\right)\right)\right]}{2 \mu R^{2} \sin \left(\mu \delta_{\max }\right)}
$$


where $\mu$ is given from equation (4-124) and $\delta_{0}$ is given by equation (4-144).

The "error" force functions of equation (4-145) and (4-146) are plotted with the quadratic force (equation (4-50)) in Figure 4.20 for $0 \leq \delta \leq \delta_{\max }$. This corresponds to the range through which $\delta_{\mathrm{l}}$ varies from minimum to maximum joint stiffness. The boundary conditions of the error function $\xi$ are $\xi_{0}= \pm 1(100 \%)$ at $\Delta_{0}=90$ degrees. As in Section 4.3.1, these equations only represent force functions for which the relationship between joint stiffness and joint deflection is independent of the set stiffness. That is, if the joint stiffness doubles for a given deflection, it is true at any set stiffness.

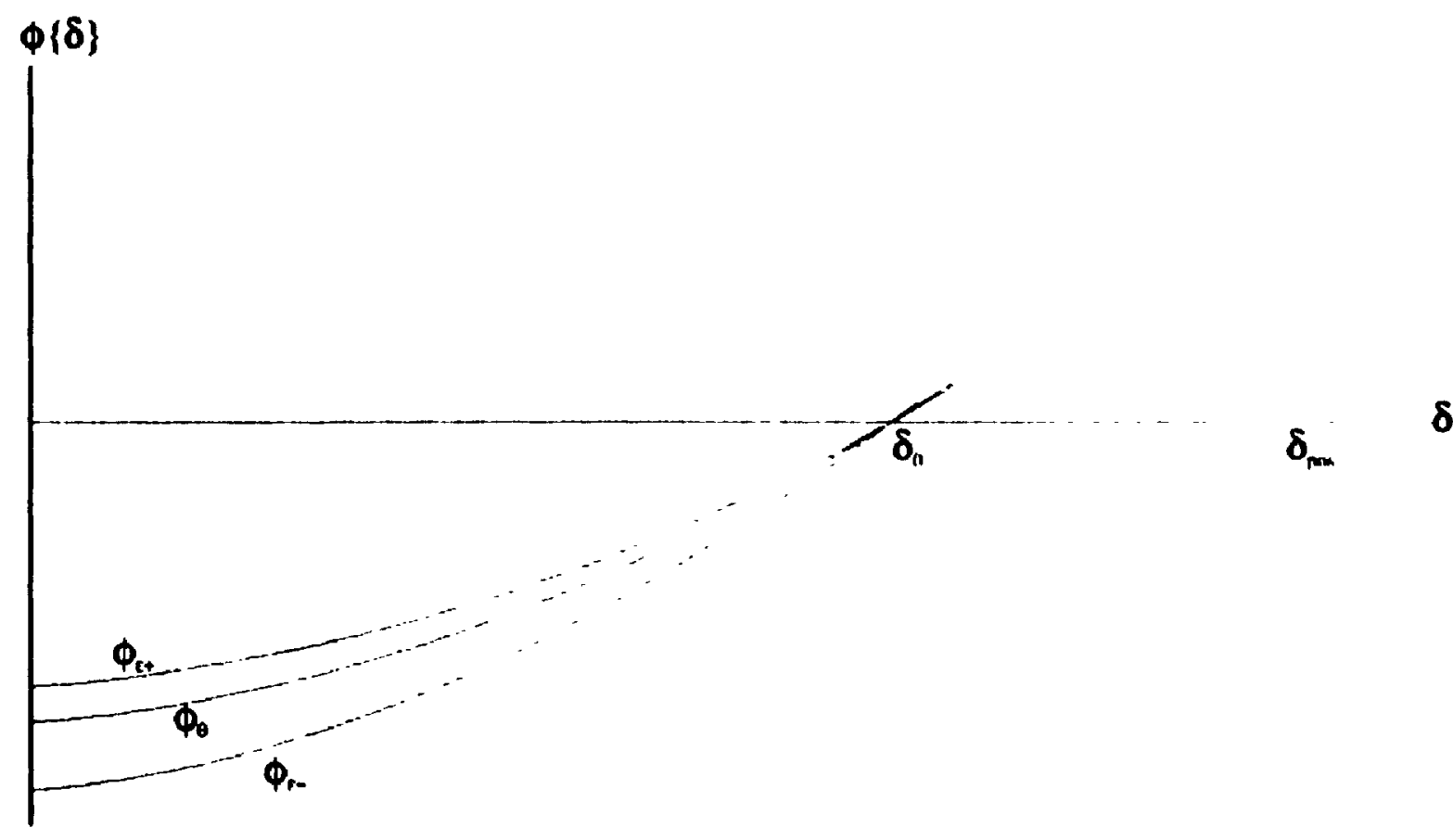

Figure 4.20: Comparison of force functions

Figure 4.20 shows that the "negative error" force function produces larger absolute forces than the quadratic function. This means that no power savings can be realized by springs that produce a negative stiffness error (reduction of joint stiffness for endpoint deflections). In fact, such stiffness elements would require more power to vary 
the stiffness. However, the "positive error" force function shows decreased force for all $\delta$ within the given range. Substituting the "positive error" function of equation (4-145) and quadratic function of equation (4-50) into equation (4-140), the power saving index is

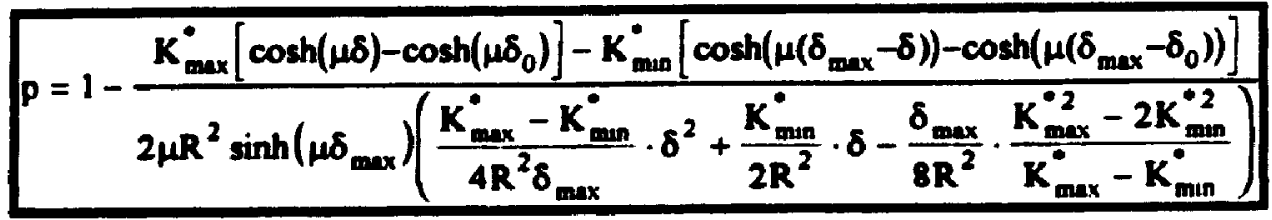

where $\mu$ is given from equation (4-118) and $\delta_{0}$ is given by equation (4-144). Figure 4.21 shows the power savings index variation with $\delta$. The maximum savings value, $p_{\max }$, depends upon the parameters chosen for the design. Such selection is performed in Chapter 6 for a prosthetic arm model. Using those values, the maximum power savings for $\xi_{0}= \pm 1(100 \%)$ at $\Delta_{0}=90$ degrees is approximately $10 \%\left(p_{\max }=0.1\right)$.

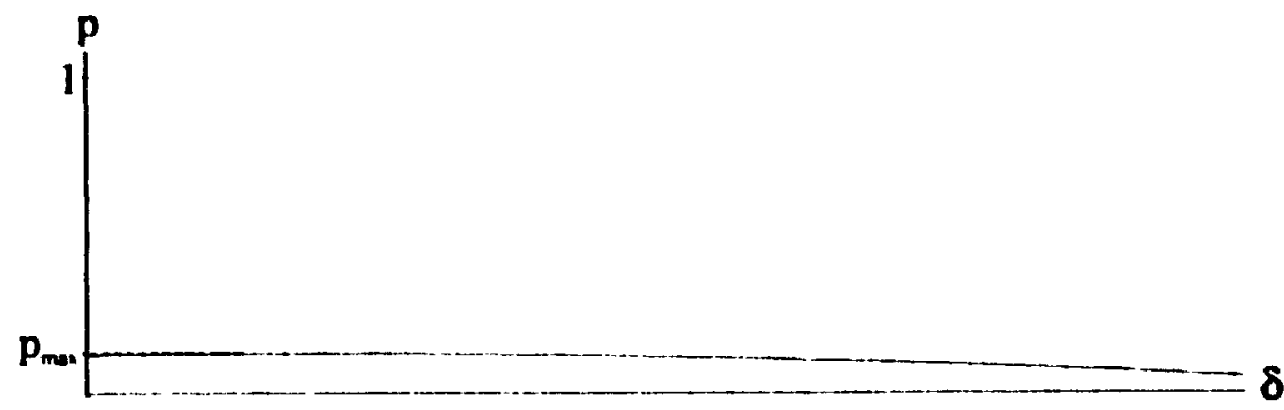

Figure 4.21: Power savings over quadratic equation.

In terms of joint operation, increasing joint stiffness means stretching the springs. The actual power requirement for a given point in time depends on the stretch at that point, however the overall maximum savings can be seen from Figure 4.21. 


\subsubsection{Neutral Force Configuration}

Section 4.5.1 shows that energy can be saved by using equal and opposite forces to directly oppose each other rather than applying them to the actuators. Configuration 2 (Figure 4.7) eliminates the need for energy to perform unloaded motion. This implies that there may be some such configuration that can eliminate the net forces created on the stiffness actuator. However, such a configuration is not as apparent. For unloaded motion, there is no net change in the energy state of the system. A quasi-static analysis of Configurations 1 and 2 show that the energy stored within the system is identical for both cases since the stretch of each spring does not change. However, for a change in joint stiffness, both springs change in displacement. A quasi-static analysis of the system, before and after a stiffness change, shows that the energy state is different. The energy stored in the springs depends upon their stretch. An increase in stiffness, which stretches the springs, increases the stored energy. In order to cancel the net force on the stiffness actuator, there must be a device that can store and supply the energy required by the springs. As previously mentioned, this can take the form of a generator that stores the energy electrically. However, such devices that work as an actuator and generator are only experimental and also involve energy losses. Mechanical storage elements include inertias and springs. Creating an inertial storage device is complicated, may contain losses due to damping, and may change the apparent mass of the system by gyroscopic effects. A spring, on the other hand, can store energy statically. In addition, the force function of an additional spring can be directly related to the branch springs with which it must interchange energy.

Figure 4.22 shows such a configuration. If the third spring is designed such that the force is always equal and opposite to the sum of the branch spring forces, there is no net force on the stiffness actuator. However, the third spring is only a function of $x_{k}$ (see Figure 4.22). Since $x_{k}$ does not change for deflections of the joint, this additional spring 
cannot detect forces created by endpoint deflection. However, in an unloaded situation the forces in the branch springs are due only to $x_{k}$, i.e., $\phi\left\{\delta_{1}\right\}=\phi\left\{\delta_{k}\right\}$ and $\phi\left\{\delta_{2}\right\}=\phi\left\{\delta_{k}\right\}$, where $\delta_{k}$ and $x_{k}$ are numerically equivalent. Thus spring 3 can be designed such that it creates an equal and opposite force of the branch springs for unloaded situations.

Following this reasoning, the force function of spring 3 should be

$$
\phi_{3}\left\{x_{k}\right\}=2 \phi\left\{\delta_{k}\right\}
$$

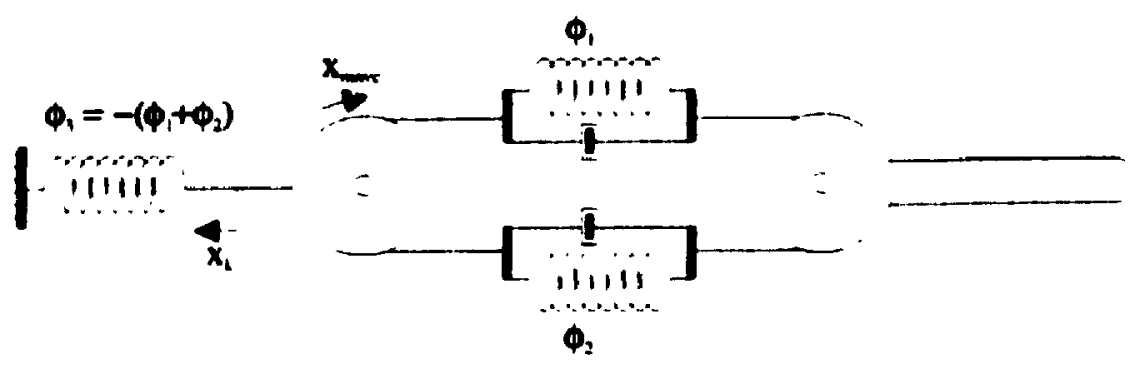

Figure 4.22: Neutral stiffness force configuration.

When the joint is loaded, one of the branch springs stretches further while the other decreases in displacement. This means that one force increases while the other decreases. In general, for non-linear springs, these two force changes are not identical. However, the stiffness actuator will only have to deal with this net change in the sum of the spring forces rather than the total sum of forces. This significantly reduces the net energy and power requirements from external sources. One drawback of using this system is the extra space required for the additional spring. 


\section{Chapter 5}

\section{Implementation}

The difficulty with implementing the design presented in this thesis is the creation of the springs. Other forms of antagonistic actuation typically focus on the method of actuation. The force-deflection curve of the stiffness elements tends to be a result of the actuator properties and not specifically designed to meet some criteria. Often the functions are not even analyzed or modeled; it is simply noted that they can control joint stiffness due to non-linearities. One notable exception is the Programmable Passive Impedance (PPI) components developed by Laurin-Kovitz et al. [1991]. They recognize the ability of quadratic springs to decouple joint stiffness and endpoint deflection and model an antagonistic actuation scheme based on these properties. The mode by which they create quadratic springs is a pre-formed metal band that approximates a quadratic force-deflection relationship. However, this approximation exists only in tension and cannot be incorporated into the quadratic function developed here by equation (4-50).

Another possible solution for developing quadratic springs is through the use of a rolamite [Wilkes, 1967, 1968; Eulert, 1968]. A rolamite, by nature, has the potential to be designed into any force-deflection function, including negative stiffness. This allows 
for the creation of the quadratic force function of equation (4-50), as well as the third spring suggested in Section 4.5.4.

The rolamite is the result of research in suspension miniaturization performed in the 1960 s by Sandia Laboratories, a division of the U.S. Atomic Energy Commission. In its basic form, a rolamite consists of a thin flexible band, two rollers, and a pair of guide surfaces (Figure 5.1). The band is essentially a spring that stores energy as it is bent. In the rolamite it is wrapped around the rollers in an S-shape thus storing energy in the curved section. The resulting restoring spring force opposes this bending and attempts to straighten the band. The symmetrical nature of the S-shape creates equal restoring forces and so the band remains in equilibrium in the direction along its length. The rollers allow the addition of tension in the band as well as provide a location for external attachment of moving parts. The guide surfaces prevent the normal component of the restoring forces from straightening the band. Moving the rollers causes them to rotate and moves the S-shaped section of the band with them. Since there is no sliding between surfaces, the resulting motion has very little friction. This phenomenon is the primary characteristic for which the rolamite was originally developed as a low friction bearing.

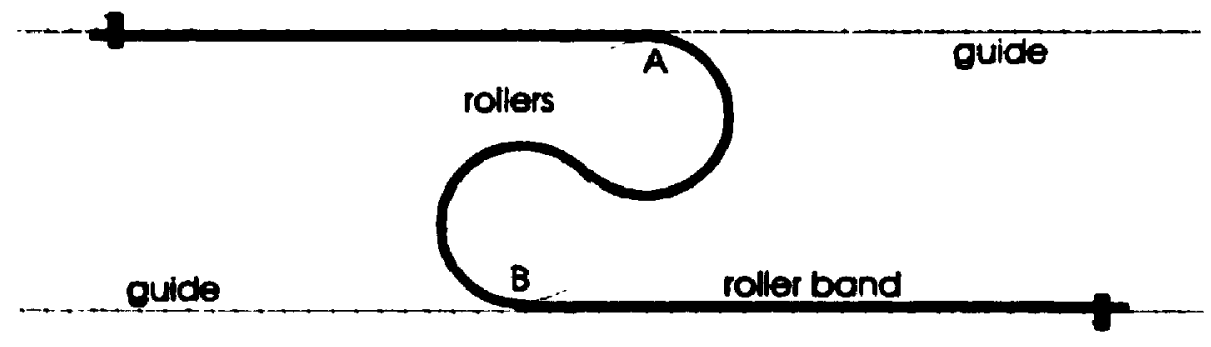

Figure 5.1: Basic rolamite configuration. 


\subsection{Force Generation in Rolamites}

The restoring force of the band is the same as that of a cantilever beam, meaning that it is a function of the cross sectional area and the modulus of elasticity of the material. If these band characteristics differ at the two centact areas $A$ and $B$ (Figure 5.1), the restoring forces are no longer in equilibrium and the side with the higher force straightens out. This moves the band and rollers. The rollers tend to rotate with little or no slippage depending on the tension within the band. As the rollers rotate, the contact points $A$ and $B$ move down the band. As the contact points change, the restoring forces can vary between them. Thus the net restoring force can vary along the length of the band.

There are three possibilities for designing the force-deflection function for the bands: material variations, thickness variation, and width variation. Changing the material of the band is impractical for most applications, especially for continuously changing force. The band must be thin to wrap around the rollers. This means that designing changes in thickness is difficult and requires high precision. As well, the restoring force is proportional to the cube of the thickness so even small errors become magnified [Wilkes, 1967; Cadman, 1969, 1971]. The easiest way to vary the force is by changing the total width of the band at the contact points as they move down its length. This can be done by removing material from the outer edges or cutting a section out of the middle. Several examples of band shapes and their force-deflection curves are shown in Figure 5.2 [Chironis, 1967].

The relationship between axial force and band width variation for a tight geometry and identical diameter rollers is given by [Wilkes, 1967, 1968]

$$
F=\frac{E t^{3}\left(w_{B}-w_{A}\right)}{6 d^{2}}
$$




$$
\begin{aligned}
& \text { where } E=\text { modulus of elasticity } \\
& t=\text { band thickness } \\
& w_{A}, w_{B}=\text { width of band material at contact points } A \text { and } B \\
& d=\text { diameter of rollers }
\end{aligned}
$$
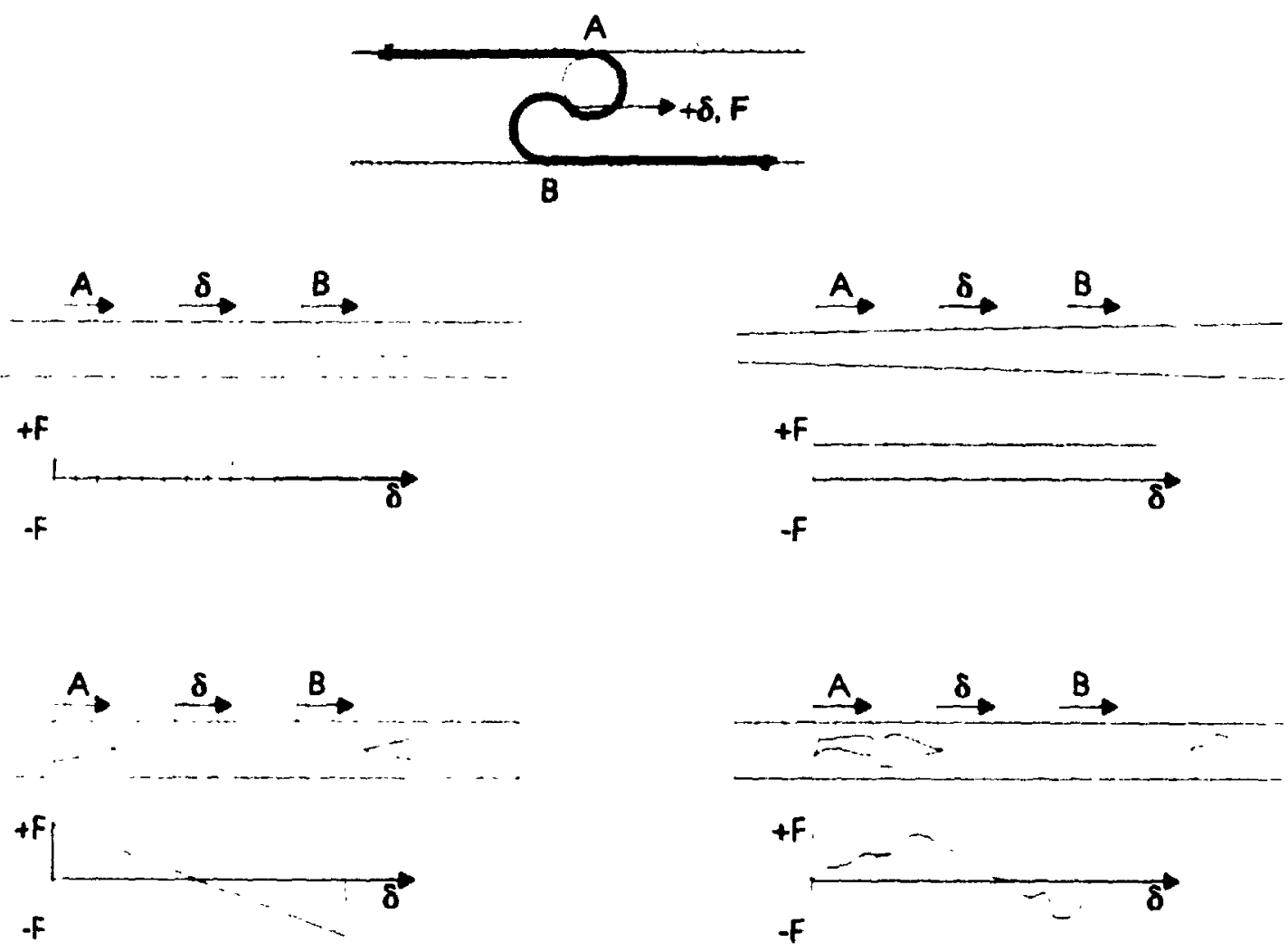

Figure 5.2: Band shapes and force curves

A tight geometry means that the tension in the band is sufficient to pull the band tangent to the rollers at points $A$ and $B$. In reality it would require infinite tension to meet this geometry. However, an effectively tight geometry can be achieved when the r.ind tension is [Wilkes, 1968] 


$$
\mathrm{T} \geq \frac{2 \mathrm{EI}}{\mathrm{td}}
$$

where $T=$ band tension

$$
\text { I = cross-sectional moment of inertia }
$$

Variations of equation (5-1) exist for looser geometries as well as different sized rollers. However, accuracy of predicted forces increases with tension, and these forces can be optimized by using identical diameter rollers. Therefore equation (5-1) is used here in developing rolamite springs. It should be noted, however, that most inaccuracies occur when band cutouts pass between the two rollers [Cadman, 1969]. This should be cunsidered when designing a rolamite spring.

\subsection{Quadratic Spring Design}

Section 4.1.3 shows that quadratic springs are desired for use in the antagonistic actuation design developed in this thesis. The ideal spring force-deflection curve for decoupling joint stiffn $s$ s and endpoint deflection is given by equation (4-50). The forcedeflection relation created in a rolamite is given by equation (5-1). Equating these two relations,

$$
\frac{E t^{3}\left(w_{B}-w_{A}\right)}{6 d^{2}}=\frac{K_{\max }^{\cdot}-K_{\min }^{\cdot}}{4 R_{e}^{2} \cdot x_{k \max }} \cdot \delta^{2}+\frac{K_{\min }^{\cdot}}{2 R_{c}^{2}} \cdot \delta-\frac{x_{k \max }}{8 R_{c}^{2}} \cdot \frac{K_{\max }^{\cdot 2}-2 K_{\min }^{\cdot 2}}{K_{\max }^{\cdot}-K_{\min }^{\circ}}
$$

Rearranging to solve for band width variation,

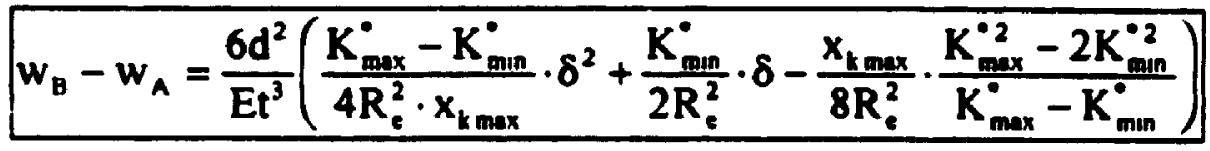

There are many band shapes through which equation (5-3) can be realized. Some of these are shown in Figure 5.3. 

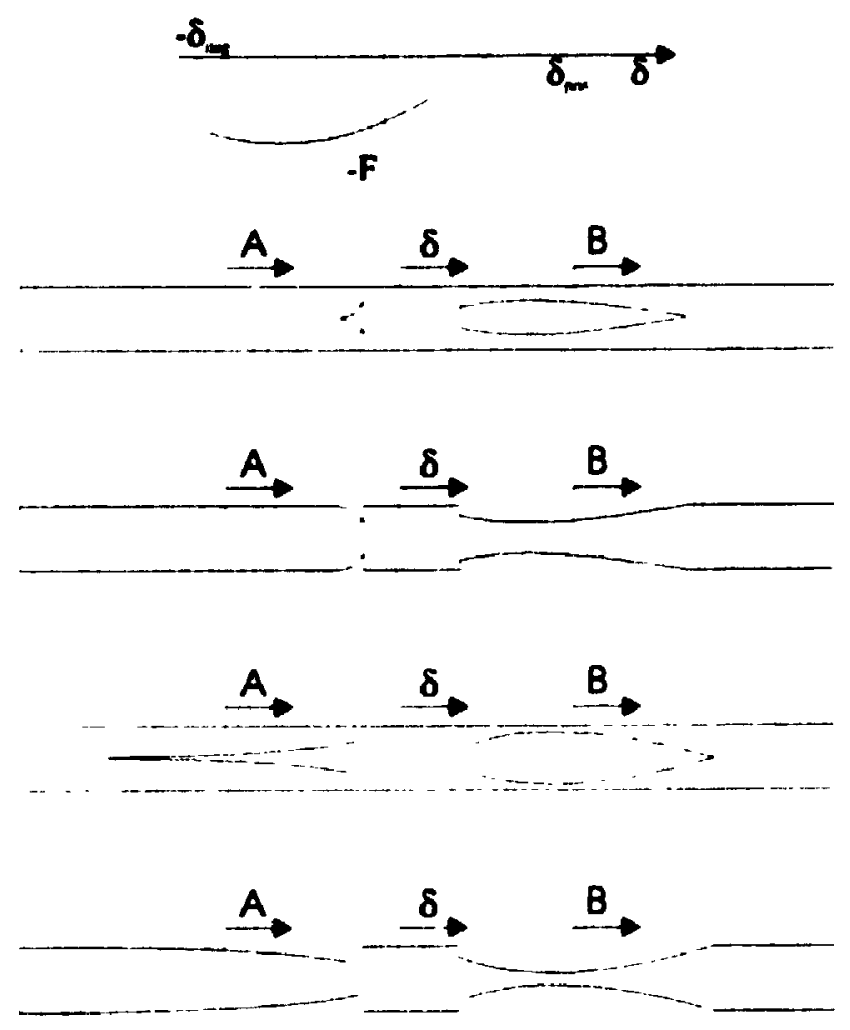

Figure 5.3: Quadratic spring rollerband shapes

\subsection{Limitations of Rolamite}

The roller-band is stressed in both tension and bending. The maximum bending and tensile strains at any given point have been shown to be [Eulert, 1968; Cadman, 1969]

$$
\begin{aligned}
& \varepsilon_{L}=\frac{t}{2 r}=\frac{t}{d} \\
& \varepsilon_{1}=\frac{T}{E W t}
\end{aligned}
$$

where

$\varepsilon_{b}=$ maximum bending strain (outer fibers of bent material) 
$\varepsilon_{1}=$ tensile strain

$w=$ width of band material at given point

$r=$ radius of rollers

The maximum stress is located on the outer surface of the bent section of the band, and is the sum of maximum bending stress and tensile stress. Expanding the above strains into stresses, the maximum stress is

$$
\sigma=\frac{E t}{d}+\frac{T}{w t}
$$

Nute that this stress does not take into account stress concentrations due to the band cutout, and is therefore an underestimate of the maximum stress.

The maximum allowable stress can be set equal to the yield stress for minimal use of the rolamite. However, as the band passes around one roller and then the other, it is flexed back and forth. An endurance stress limit should therefore be chosen based on a fatigue life analysis for repeated use of the rolamite. This fatigue stress limit is a property of the material and such graphs are widely available. Setting equation (5-4) equal to this endurance stress limit,

$$
\sigma_{c}=\frac{E t}{d}+\frac{T}{w t}
$$

where $\sigma_{c}=$ endurance stress linit based on fatigue life. Rearranging equation (5-5) into quadratic form in relation to the band thickness,

$$
E t^{2}-\sigma_{e} d t+\frac{T d}{w}=0
$$

The solutions to equation (5-6) are

$$
t_{\max }=\frac{d}{2 E}\left(\sigma_{e}+\sqrt{\sigma_{e}^{2}-4 \frac{E T}{w d}}\right)
$$




$$
t_{\min }=\frac{d}{2 E}\left(\sigma_{c}-\sqrt{\sigma_{c}^{2}-4 \frac{E T}{w d}}\right)
$$

where $t_{\max }$ and $t_{\min }$ are the maximum and minimum allowable band thicknesses, respectively. These limits are shown on a plot of equation (5-4) in Figure 5.4.

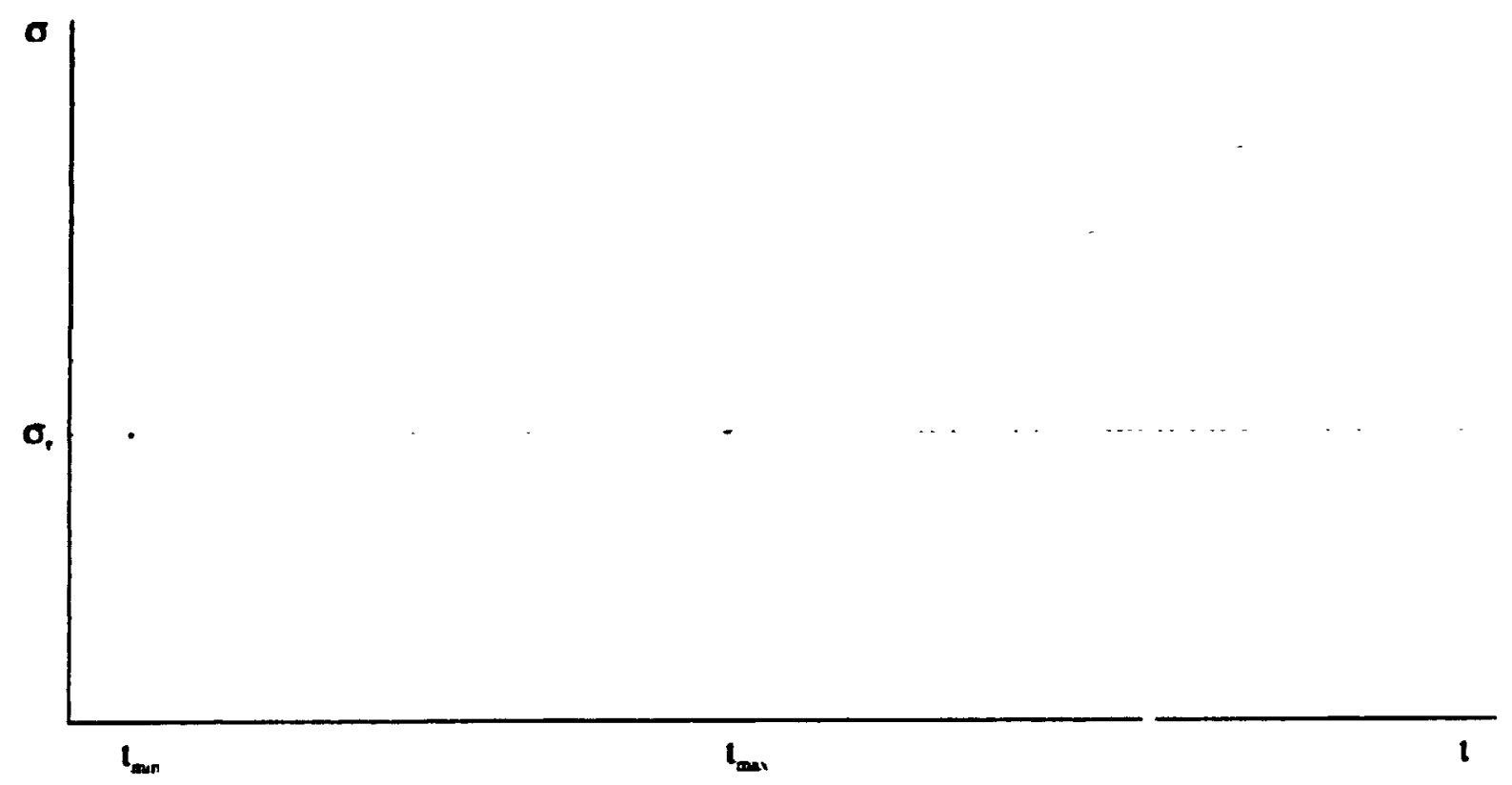

Figure 5 4: Maximum stress as a function of band thickness.

From Figure 5.4 it can be seen that there is an optimal band thickness that produces the least amount of stress. This would be the ideal thickness to choose if one wanted to maximize the tension, and thus accuracy, or reduce the size of the rolamite (smaller roller diameter or band width). However, the force in a rolamite, from equation (5-1), suggests that in order to maximize force one must maximize the relation $t^{3} / d^{2}$. Thus for any given diameter $d$ one would want the maximum thickness allowed. The relationship is given by equation (5-7). A graph of this relationship (Figure 5.5) shows 
that $t_{\max }$ decreases for decreasing diameter $d$. This means that the thickness should be as large as possible.

Equation (5-7) is maximized by large maximum stress $\left(\sigma_{c}\right)$, greater width (w), low modulus of elasticity (E), and low tension ( $T$ ). This also makes sense by the following reasoning: allowing higher stress increases the limitation in Figure 5.5, greater width provides more cross-sectional area to support the tension forces, a low modulus of elasticity means the material is more flexible and thus can handle higher bending forces before yielding, and lower tension creates less stress in the outer fibers so the band can be thicker.

As previously mentioned, lower tension means less accuracy. The minimum allowable tension to maintain a tight geometry is given by equation (5-2). The area moment of inertia for a rectangular cross-sectional area is

$$
I=\frac{1}{12} w^{3}
$$

Substituting equation (5-9) into (5-2),

$$
T \geq \frac{E w^{2}}{6 d}
$$

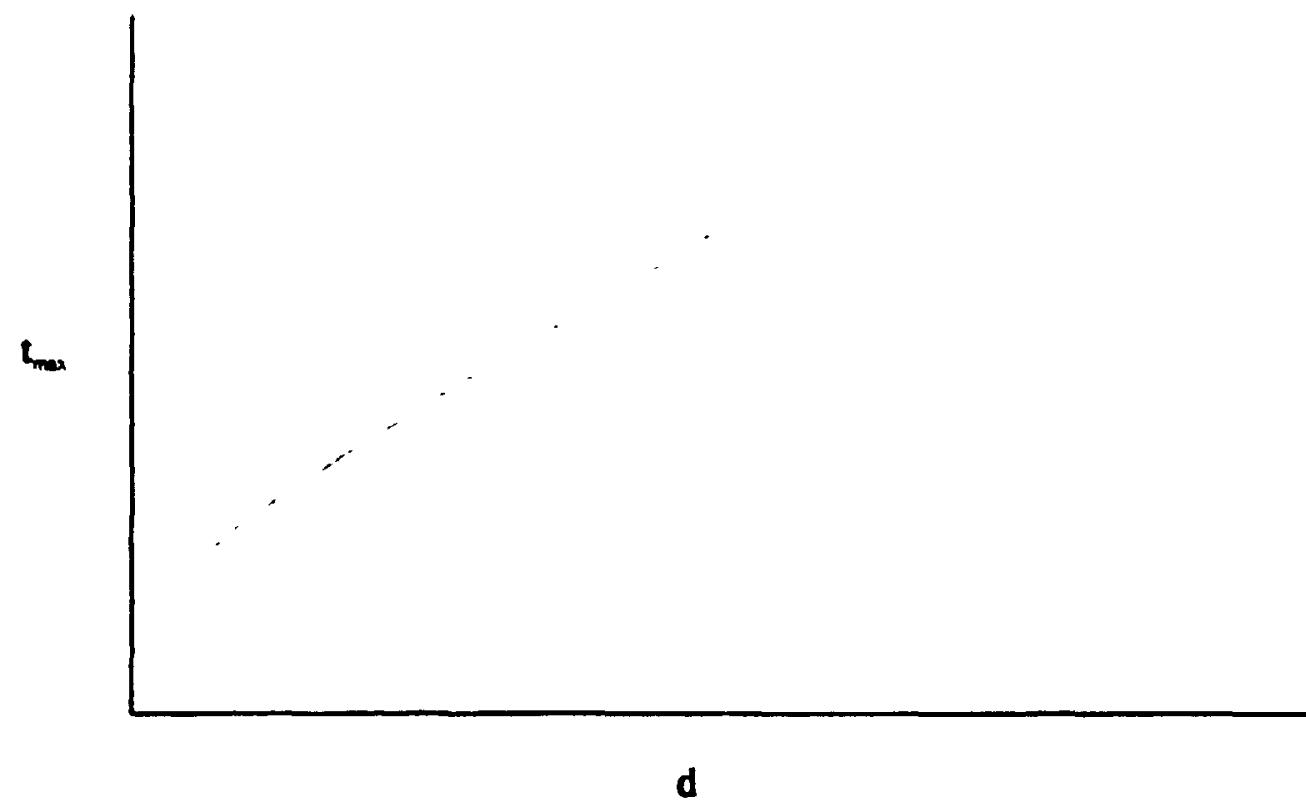

Figure 5.5. Maximum thickness vs. roller diameter 
At the minimum tension of equation (5-10), equation (5-6) becomes

$$
E t^{2}-\sigma_{e} t d+\frac{1}{6} E t^{2}=0
$$

Simplifying and rearranging,

$$
t=\frac{6 \sigma_{e} d}{7 E}
$$

Substituting equation (5-11) into (5-1),

$$
F=\frac{36}{3} \mathrm{~d}\left(w_{B}-w_{A}\right)\left(\frac{\sigma_{c}^{3}}{E^{2}}\right)
$$

The last term of equation (5-12) is defined at the force factor of the material [Bell, 1968]. Both $\sigma_{c}$ and $\mathrm{E}$ are properties of the band material so the force factor can be tabulated. Proper band material is chosen based on force factor characteristics. The remaining two terms define the cross-sectional size of the rolamite device. The overall width of the band is given by $w_{B}$. Maximum force is achieved when $w_{B}$ is largest and $w_{A}$ is smallest. However $w_{A}$ must be greater than zero width because there must be some material to hold the band together. Thus for maximum force purposes $w_{B}$ and $w_{A}$ can be redefined as $w_{\max }$ and $w_{\min }$, respectively. The maximum force that can be produced by a rolamite then becomes

$$
F_{\text {max }}=\frac{36}{3} \mathrm{~d}\left(w_{\max }-w_{\min }\right)\left(\frac{\sigma_{e}^{3}}{E^{2}}\right)
$$

The limitations that this creates in the antagonistic actuation design can be found by substituting this maximum force into $\alpha$ in equation (4-79).

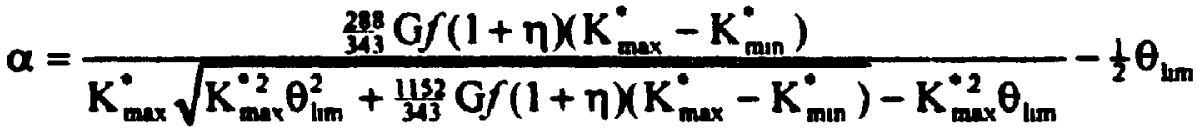

$$
\begin{aligned}
& G=d\left(w_{\max }-w_{\max }\right)\left(l_{b m}\right) \\
& f=\frac{\sigma_{c}^{3}}{\mathrm{E}^{2}}
\end{aligned}
$$


The limiting torque equations remain unchanged from Section 4.2.2. The positive torque limitation associated with spring 2 reaching maximum stretch is

$$
T_{\mathrm{lum}}=\mathrm{K}_{\max }^{*} \Delta_{\mathrm{lmm}}-\frac{\left(\mathrm{K}_{\max }^{\prime}-\mathrm{K}_{\min }^{\prime}\right)(1+\eta) \Delta_{\mathrm{lm}}^{2}}{2 \alpha}
$$

The corresponding negative torque limit associated with spring 1 reaching maximum stretch is

$$
\mathrm{T}_{\mathrm{hm}}=\frac{\left(\mathrm{K}_{\max }^{*}-\mathrm{K}_{\min }^{*}\right)(1+\eta) \Delta_{\mathrm{hm}}^{2}-\mathrm{K}_{\max }^{*} \Delta_{\mathrm{hm}}}{2 \alpha}
$$

The positive torque limit associated with spring 1 reaching maximum compression is

$$
T_{\mathrm{lim}}=\frac{\left(K_{\max }^{*}-K_{\min }^{*}\right)(1+\eta) \Delta_{\mathrm{hm}}^{2}}{2 \alpha}+\left((1+\eta) K_{\min }^{*}-\eta K_{\max }^{*}\right) \Delta_{\mathrm{lmm}}
$$

The corresponding negative torque limit associated with spring 2 reaching maximum compression is

$$
T_{\mathrm{lim}}=\left(\eta \mathrm{K}_{\max }^{\cdot}-(1+\eta) \mathrm{K}_{\min }^{\cdot}\right) \Delta_{\mathrm{lm}}-\frac{\left(\mathrm{K}_{\max }^{\circ}-\mathrm{K}_{\operatorname{mun}}^{\cdot}\right)(1+\eta) \Delta_{\mathrm{hm}}^{2}}{2 \alpha}
$$

The design of the joint mechanism now falls into three factors. Geometric factor G (equation (5-15)) determines the size of the rolamite (height, width, length), factor $f$ (equation (5-16)) is the force factor of the roller-band and depends upon the choice of materials, and all other remaining parameters in equations (5-14), (4-82), (4-83), (4-85), and (4-86) define the abilities of the joint. Thus size restrictions are traded off for performance. In order for the rolamite to be adequate for use in this design, minimum performance abilities must be met within a maximum defined size restriction. 


\section{Chapter 6}

\section{Application of Design}

In order for a design to have meaning, it must have real world applications. In this chapter, typical real world values are chosen as design parameters. The sensitivity of the design on the major parameters, such as size, is demonstrated for fexibiiity of the parameter selection. The torque limitations given in Section 4.2.2 for general operation, and Section 5.3 for rolamite application, provide a measure of performance for the joint. The joint can perform all required functions if the limits of operating criteria fall within the stiffness control region (Figure 4.11(b)). Simulations of the design for a variety of tasks, showing the resulting characteristics and abilities, are another measure of design performance.

\subsection{Selection of Parameters}

Equations (4-82), (4-83), (4-85), and (4-86) require the selection of six parameters: $\eta, I_{\text {hm }}, K_{\text {max }}^{*}, K_{\text {man }}^{*}, \theta_{\mathrm{hm}}$, and $F_{\max }$. The variation of $\eta$ results in clipping of the 
maximum deflection at lower stiffness (Figure 4.10(b)), and a minor increase in maximum limiting torque. Section 4.2 .1 shows that the practical limits of $\eta$ are between 0 and 1 . It is typical that one reduces joint stiffness when in contact with a constraint. Thus high contact forces and deflections are required for low stiffness. According to the analysis of Section 4.2 .2 , this suggests that it is desirable to have $\eta=1$.

The maximum length of space available, $l_{\mathrm{hm}}$, depends upon the application of the design. One of the intended applications of this thesis is in upper limb prostheses. Therefore the size limitations of a human forearm will be used as an example. It is assumed that the forearm mechanical components have a limitation of $25 \mathrm{~cm}$ in length. Ideally, the springs will only take up a small portion of this. The limiting length of the springs is therefore taken as $5 \mathrm{~cm}$.

Continuing with the prosthesis application, the limits of $\mathrm{K}_{\max }^{{ }_{\max }}$ and $\mathrm{K}_{\min }$ are a function of human elbow joint abilities. Research suggests that the human range of stiffness is between $1 \mathrm{~N} \cdot \mathrm{m} / \mathrm{rad}$ and $200 \mathrm{~N} \cdot \mathrm{m} / \mathrm{rad}$ [Hogan, 1985b; Abul-Haj \& Hogan, 1990a]. These are the ideal limits for a prosthesis design. However, these are extremes. The normal operation range tends to be a little more narrow. An acceptable minimum operating stiffness is $7 \mathrm{~N} \cdot \mathrm{m} / \mathrm{rad}$ [Mussa-Ivaldi et al., 1985; Abul-Haj and Hogan, 1990]. Reaching maximum stiffness (tensing all muscles in the arm) usually results in vibration and shaking. This high stiffness is far greater than required in normal arm tasks. It is assumed that high stiffness range of normal arm motions occurs between $50 \mathrm{~N} \cdot \mathrm{m} / \mathrm{rad}$ and $80 \mathrm{~N} \cdot \mathrm{m} / \mathrm{rad}$. Noting from the geometric limitations (Section 4.2) that the joint becomes effectively rigid at maximum stiffness, choosing the lowest value for $K^{{ }_{\text {max }}}$ as $80 \mathrm{~N} \cdot \mathrm{m} / \mathrm{rad}$ allows the effective maximum stiffness to fall within the acceptable range.

Elbow rotations have a limit of approximately 140 degrees. However, a simple test can show that a normal human elbow can create torque when fully flexed or extended. This means that the equilibrium position must be beyond the physiological limits of the arm. It is assumed that the equilibrium position can vary up to 30 degrees 
beyond the physiological limits, thus the operational range of the arm, $\theta_{\lim }$, is 210 degrees [Abul-Haj and Hogan, 1990].

The maximum force, $F_{\text {mox }}$ achievable by the springs depends upon the method of creating the springs. At this point, the only known methods of creating quadratic springs are through the PPI components developed by Laurin-Kovitz et al. [1990] and the rolamite. However, the PPI spring is only quadratic over a small range of stretch and does not continue to be quadratic in compression. Thus it does not fit the quadratic profile required in this design. The rolamite, however, can be designed around any force function for any given deflection. The effect of introducing the rolamite into this design has already been shown in Chapter 5 . This effectively replaces the $F_{\max }$ term with the roller diameter $d$, the maximum difference of band width $\left(w_{\max }-w_{\min }\right)$, and the force factor $f$ of the band material.

The two rolamites must fit within the bounds of the forearm. It is assumed that they must each be able to fit within $\mathrm{a} \mathbf{3} \mathrm{cm}$ by $3 \mathrm{~cm}$ cross section. Assuming minimal casing thickness this means that the maximum allowable roller diameter is $3 \mathrm{~cm}$. On the same assumption the maximum width of the band, $w_{\max }$, is also $3 \mathrm{~cm}$. The minimum width requires enough material to hold the force within the band. However, the force and minimum width are dependent on each other. Choosing the minimum width determines what the maximum force can be, and vice versa. A limit on this relationship is a minimum width of zero. No force can be created above the threshold defined by a minimum width of zero. Ideally, the rolamites would be smaller, perhaps about $1 \mathrm{~cm}$ square in cross section.

The choice of materials for the roller-band is based on the best force factor.

Before choosing force factors, one must first determine the acceptable stress limit. For a one shot activation of the rolamite, the yield stress of the material will suffice. However, in this application the band goes through repeated flexure, so fatigue life plays an important part in determining an endurance stress. Compiled data on common spring 
materials [Bell, 1969] lists a maximum infinite life force factor of $2.66 \mathrm{psi}$ (18.3 kPa) for Titanium alloy (Ti-6Al-4V). The maximum one shot force factor is $46.6 \mathrm{psi}$ ( $321.4 \mathrm{kPa}$ ) for Sundvik 11R51 Steel. Ideally the bands would have infinite life. However, existing prostheses must be maintenanced every few months. This provides an opportunity to replace the rolamites before failure. As a minimum requirement, it is assumed that they must go through 1000 cycles/day for 120 days for a cycle life of 120,000 . Using the fatigue life plot for Titanium 6-4, this results in an endurance stress of approximately 95 ksi (655 MPa). Using the modulus of elasticity of $16.5 \times 10^{6} \mathrm{psi}(113.8 \mathrm{GPa})$, the force factor is $3.15 \mathrm{psi}(21.7 \mathrm{kPa})$. It should be noted that the data is from 1969 and more modern materials may have force factors that surpass the ones presented here as an example. Table $6 \mathrm{~A}$ below shows a summary of the parameter limits and ideal choices.

Table 6A: Parameter selection

\begin{tabular}{|c|c|c|c|}
\hline parameter & lower limit & upper limit & ideal \\
\hline$\eta$ & 0 & 1 & 1 \\
\hline $\mathrm{K}_{\max }^{\circ}$ & $80 \mathrm{~N} \cdot \mathrm{m} / \mathrm{rad}$ & $\infty$ & $200 \mathrm{~N} \cdot \mathrm{m} / \mathrm{rad}$ \\
\hline $\mathrm{K}_{\min }^{*}$ & $0 \mathrm{~N} \cdot \mathrm{m} / \mathrm{rad}$ & $7 \mathrm{~N} \cdot \mathrm{m} / \mathrm{rad}$ & $1 \mathrm{~N} \cdot \mathrm{m} / \mathrm{rad}$ \\
\hline$\theta_{\operatorname{lm}}$ & 0 & $\infty$ & $3.67 \mathrm{rad}$ \\
\hline $\mathbf{l}_{\lim }$ & $0 \mathrm{~m}$ & $0.25 \mathrm{~m}$ & $0.05 \mathrm{~m}$ \\
\hline$d$ & $0 \mathrm{~m}$ & $0.03 \mathrm{~m}$ & $0.01 \mathrm{~m}$ \\
\hline $\mathbf{w}_{\max }-\mathrm{w}_{\min }$ & $0 \mathrm{~m}$ & $0.03 \mathrm{~m}$ & $0.01 \mathrm{~m}$ \\
\hline$f$ & $0 \mathrm{~Pa}$ & $21700 \mathrm{~Pa}$ & $18300 \mathrm{~Pa}$ \\
\hline
\end{tabular}

Since $\eta=1$ is used, only equations (4-82) and (4-83) apply. Substituting the ideal parameters into equations (4-82), (5-14), (5-15), and (5-16) results with an stiffness limiting region (Figure 6.1) defined by

$$
T_{\text {bim }}=200 \Delta_{\text {lim }}-1910783 \Delta_{\text {lim }}^{2}
$$

Note from the graph that within the variable stiffness region the maximum torque and 


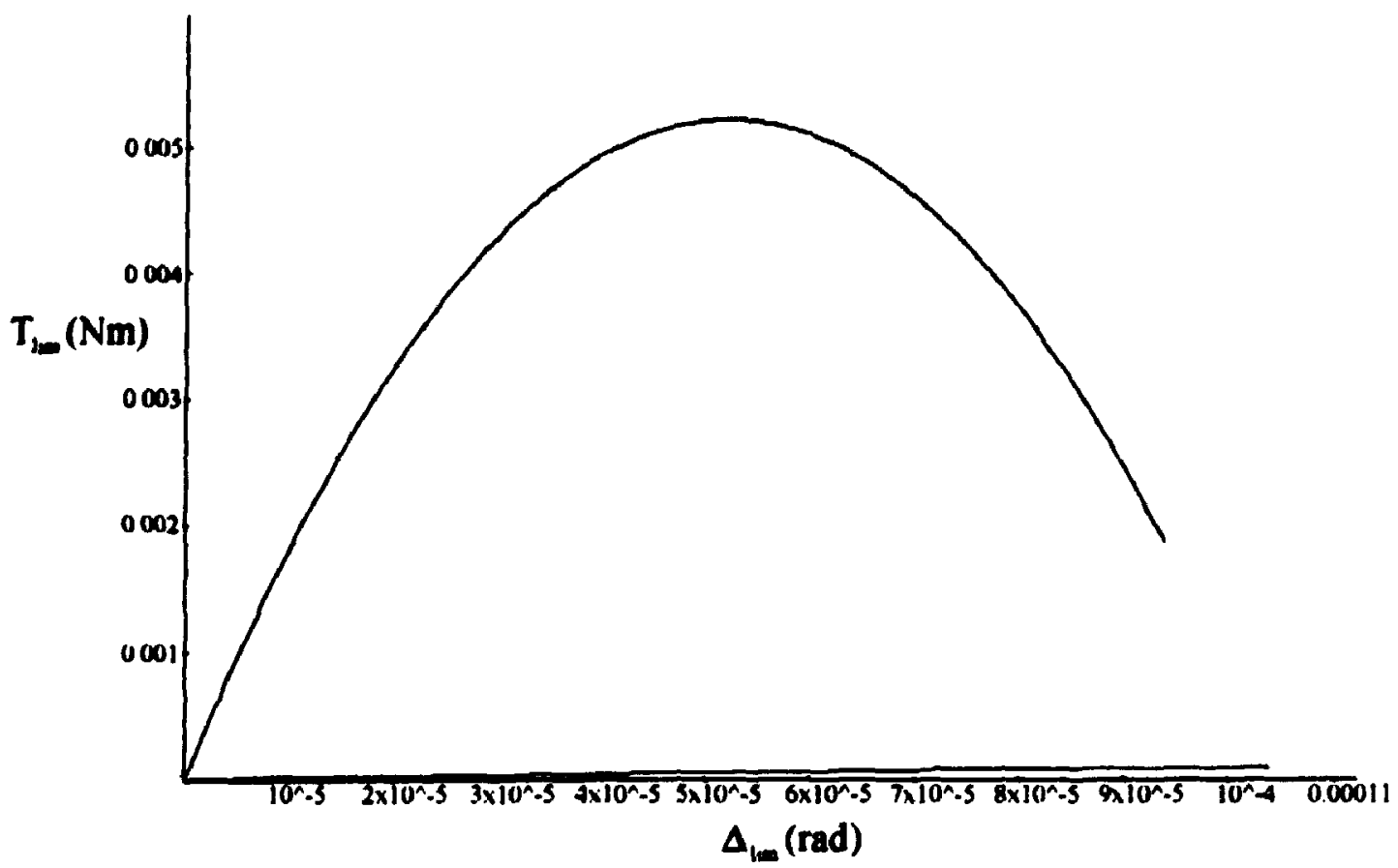

Figure 6.1: Variable stiffness region using ideal parameters

joint deflection that can be produced are approximately $5.2 \times 10^{-3} \mathrm{~N} \cdot \mathrm{m}$ and $1.04 \times 10^{-4} \mathrm{rad}$ ( 0.006 degrees), respectively. This is certainly far below desired results. It is important to realize that this is not the maximum torque that can be produced by the joint. It is the maximum torque that can be produced by a deflection of the springs. Additional torque can be created by the motors and is usually limited by the motor stall torque. However, this requires the springs to be fully deflected and the arm operating in the rigid region. The attempt of this thesis is for the actuator to function within the stiffness control region, i.e., it is not designed to be operated in the rigid region.

Variation of the parameters may allow improvements of the stiffiness control region. Figure 6.2 shows the effect of varying each parameter within the limits shown in Table 6A. Decreasing $\eta$ to 0 provides negligible increase of limiting torque and significantly reduces the deflection limit at lower stiffnesses. Therefore $\eta=1$ is the best choice. Reducing $\mathrm{K}_{\max }$ has no effect on the maximum torque but does increase the deflection limit at a better than proportional rate (as $\mathrm{K}_{\max }$ increases linearly, $\Delta_{\mathrm{lm}}$ 
increases at a greater rate). This suggests that $\mathrm{K}_{\text {max }}^{\cdot}=30 \mathrm{~N} \cdot \mathrm{m} / \mathrm{rad}$ should be used to maximize the variable stiffness region. Variation of $\mathrm{K}_{\min }{ }^{-}$has negligible effect on the limiting torque and therefore selection can be based on other criteria. Changing $I_{\mathrm{Im}}, w_{\max }$ $w_{\text {mun }}, d$, and $f$ all show linear relationships with the stiffness controlled region, i.e., doubling the parameter doubles the limiting torque and deflection limit. Thus using the largest permissible value for each is best. Decreasing $\theta_{\mathrm{hm}}$ has the most dramatic effect on limiting torque, with an increase that is more than one hundred times larger. However, reduction of $\theta_{\mathrm{lm}}$ to 0 means that the motion of the arm is impossible in the current configuration. An alteration of the configuration can solve this problem.

If the springs are stationary for motions of the arm, the effect that $\theta_{\mathrm{lum}}$ places on the system is eliminated. This requires the motion actuation to come between the springs and the joint. Such a configuration is shown in Figure 6.3. Note that it is almost identical to that of Figure 3.5(b), which is shown to be an equivalent system. The geometry of the configuration in Figure 6.3 is slightly different from that developed in Section 4.2.1. Commanded motion of the arm does not require the branches to move linearly in opposite directions as in the configurations in Figures 4.6 and 4.7. Instead, $\theta_{a q}$ is directly commanded to the position actuator between the branches and joint (see Figure 6.3). Therefore $\theta_{o q}$ is unlimited since the position actuator can rotate the joint position indefinitely. Such a configuration has the added feature that since translation of the branch components is not required, either the springs can become larger to fill up the space previously required for motion, or the size of the system can be reduced (no change in spring size). The effect that this places on the geometric analysis in Section 4.2.1 is the removal of the $\theta_{\mathrm{lim}}$ limitation, i.e., $\theta_{\mathrm{lmm}}$ effectively becomes zero in the equations. The difficulty with implementing such a configuration is the added complexity since the motor that creates the motion, along with gearing, must be able to rotate within a casing. The mechanism must be designed so that it is non-backdrivable and external torques are transmitted through the casing to the springs rather than through the motor shaft. 

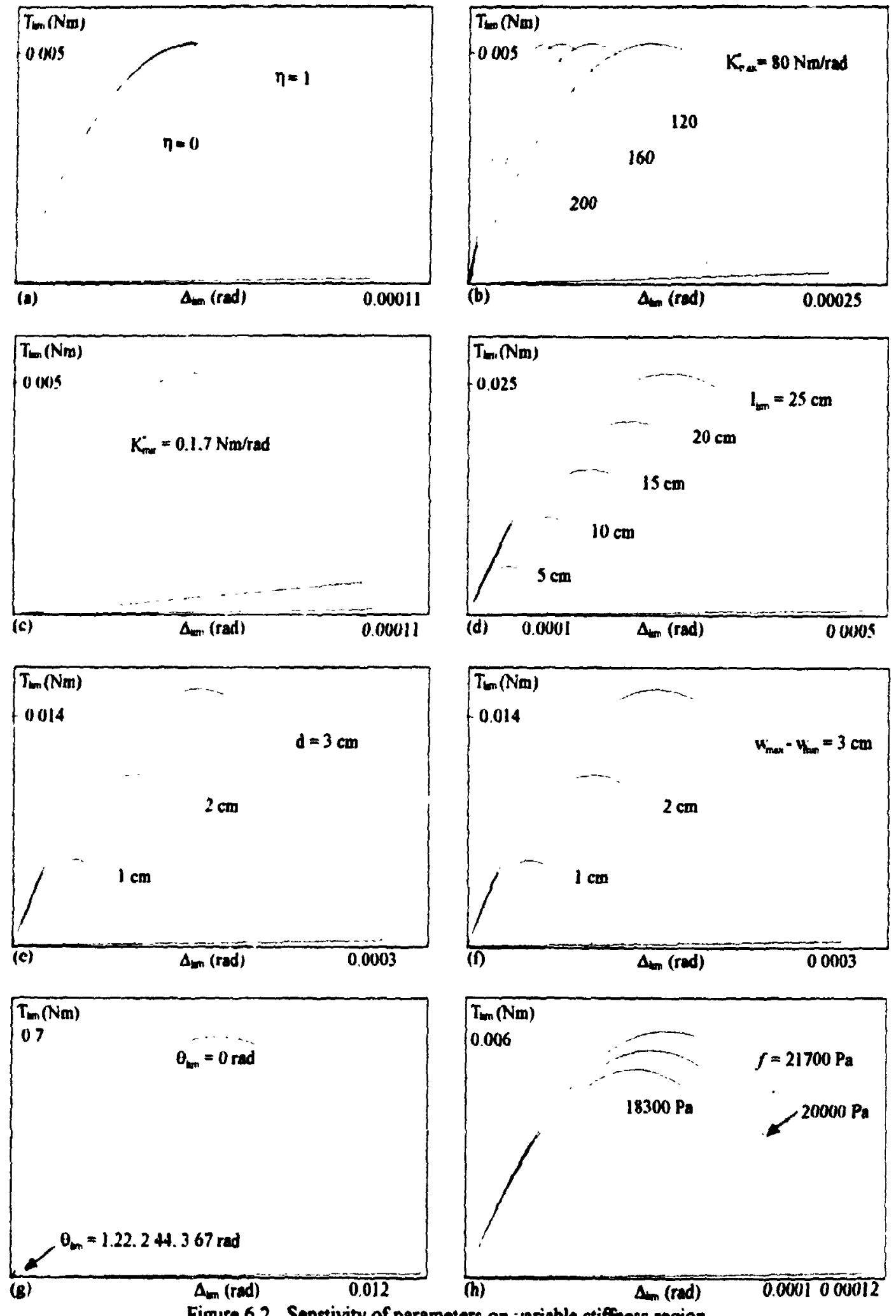

Figure 6.2 Senstivity of parameters on variable stiffness region.

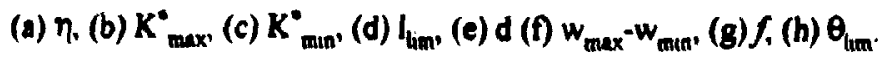




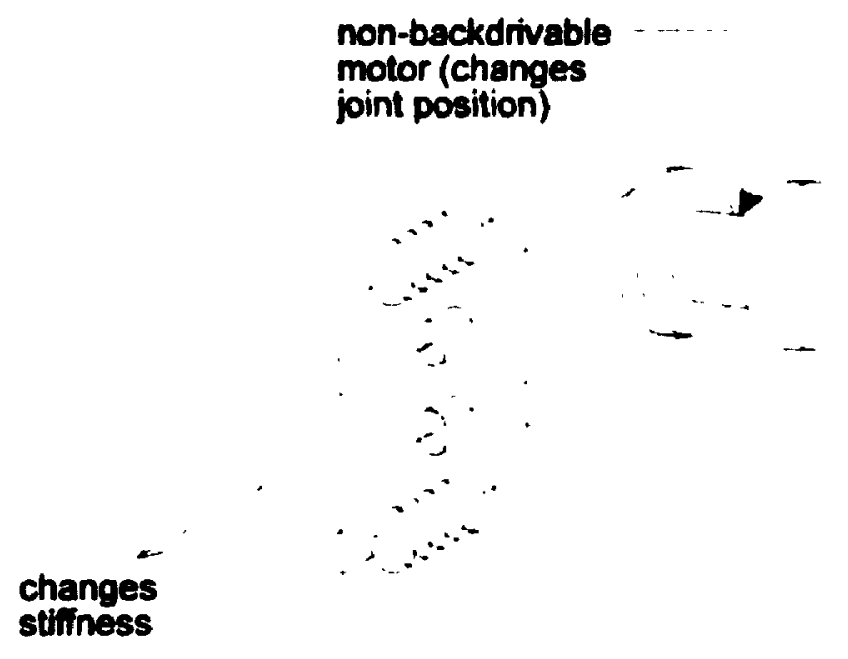

Figure 6.3. Configuration with position actuator at joint Note that arm movement is controlled by a motor between the springs and joint. thus space is not required to slide the springs

The results of using the parameter a alues that maximize the stiffness control region, as suggested above, show that a maximum limiting torque of $3.2 \mathrm{~N} \cdot \mathrm{m}$ and a deflection limit of $0.16 \mathrm{rad}$ ( 9.67 degrees) can be achieved using the rolamites (see Figure 6.4). While these are both significantly larger than initial calculations, they still do not reach acceptable limits. Existing prostheses are typically capable of operating up to $16 \mathrm{~N} \cdot \mathrm{m}$ until the stall torque of the motor is achieved. As previously mentioned, the stiffness controlled region presented here can be used in addition to the stall torque, however that is not the intention of the design. It does not appear that rolamites, in the form used here, are sufficient for use in a prosthesis application of this design. However, adaptation of the rolamite concept may provide better results. Also, they can still be used in robotic manipulators that do not have the severe size or torque requirements of a prosthetic arm. 


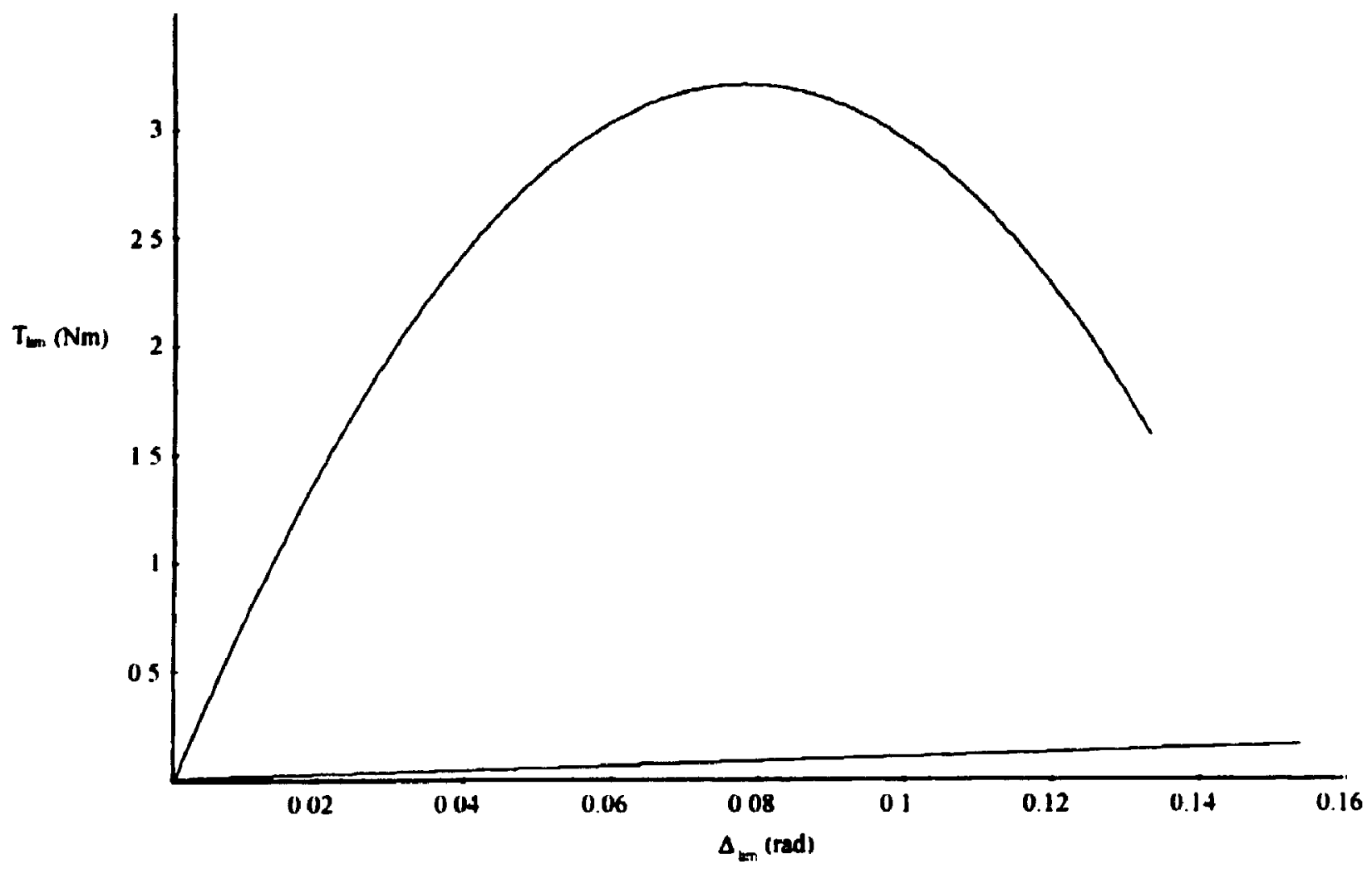

Figure 6 4. Maximized stiffness control region

\subsection{Simulation}

In addition to the parameters selected, it is also necessary to choose values for mass of the arm, arm length, damping, external loads, and the stiffness of the environment. The mass of the arm, $\mathrm{m}$, is chosen to be $280 \mathrm{~g}$, approximately equal to that of currently available prostheses. The center of gravity is chosen to be at the center of the arm, i.e., at half of the length. The arm length is chosen to be $0.33 \mathrm{~m}$, typical of an adult male forearm. Damping, external loads, and environmental stiffness depend upon the task for which the simulated arm is to perform. These are specified with the definition of each task. It is also necessary to specify the configurations to be used in the simulations. 


\subsubsection{Configuration Definitions}

The effect of introducing rolamites into the design is the replacement of the maximum spring force, $F_{\max }$, in equation (4-79) which is used in the torque limitations. Since this proved to be insufficient for the design of an upper limb prosthesis, simulation requires a change in application, or a change in the value for $F_{\max }$. A change in application calls for new parameter selection. It is simpler to remove the constraints of the rolamites. As a minimum acceptable limit, the prosthesis should be able to produce a torque of at least $16 \mathrm{~N} \cdot \mathrm{m}$ within the stiffness controlled region. This is approximately the maximum torque that current prostheses can develop. The required force to provide this torque depends upon the configuration. Only the configuration of Figure 6.3 allows the elimination of $\theta_{\mathrm{lmm}}$. For the purpose of comparison, simulations are performed using Configuration 1 (Figure 4.6), Configuration 2 (Figure 4.7), and a third configuration that combines the improvements suggested in Sections 4.5.4 (Figure 4 22) and 6.1 (Figure 6.3). However, this third configuration results in different sized springs from the first two configurations in order to produce the same maximum torque. This allows comparison of the configurations while maintaining a constant stiffness controlled region. A fourth configuration uses the same system as Configuration 3, but with identical springs to that of Configurations 1 and 2. This means that the stiffness controlled region of Configuration 4 is different from that of the other three. The purpose of this the fourth system is to compare simulations based on the same springs rather than the same output performance. The definition and parameter selection of each configuration are given below.

\section{Configuration 1}

This configuration is the original system model using separated branch actuators. A diagram of the configuration is shown in Figure 6.5. 


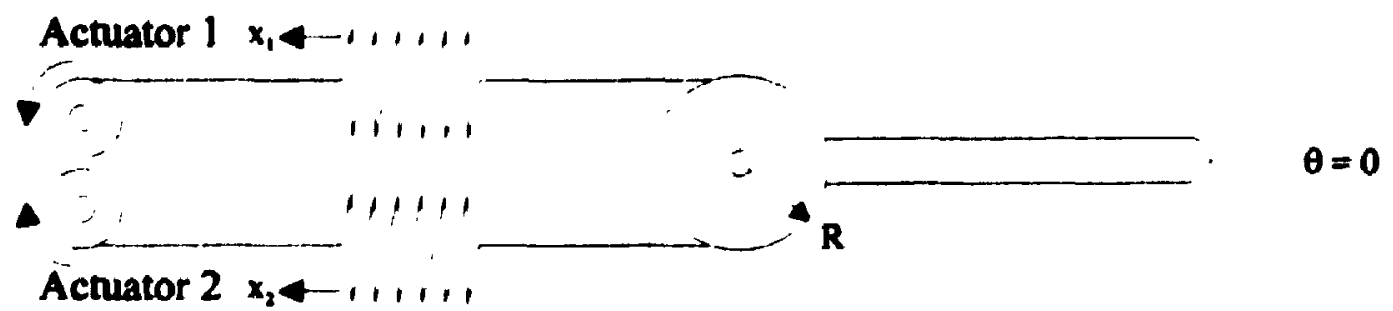

Figure 6.5. Configuration I (neglecting the dampers)

The stiffness controlled region of this configuration is severely limited by $\theta_{\mathrm{lm}}$, as seen in the previous section. The maximum limiting torque occurs when

$$
\frac{d T_{h m}}{d \Delta_{h m}}=0
$$

Using the positive torque limit for the case where $\eta=1$, the derivative of equation (4-82) is

$$
\frac{d T_{l m}}{d \Delta_{l m}}=K_{\max }^{\cdot}-\frac{\left(K_{\max }^{\cdot}-K_{\min }^{\cdot}\right)(1+\eta) \Delta_{l m}}{\alpha}=0
$$

Rearranging,

$$
\Delta_{\mathrm{lm}}=\frac{\mathrm{K}_{\max }^{\cdot} \alpha}{\left(K_{\max }^{\cdot}-K_{\min }^{*}\right)(1+\eta)}
$$

Substituting this deflection into equation (4-82) and simplifying, the maximum limiting torque is

$$
T_{\max }=\frac{K_{\max }^{\cdot 2} \alpha}{2\left(K_{\max }^{*}-K_{\min }^{\cdot}\right)(1+\eta)}
$$

where $\alpha$ is defined by equation (4-79). The maximized parameters that apply to this configuration are provided in the Table 6B below. Note that damping is chosen based on trial and error in the simulations. 
Table 6B: Chosen parameters

\begin{tabular}{|c|c|}
\hline parameter & value \\
\hline$\eta$ & 1 \\
\hline $\mathrm{K}_{\text {max }}^{\circ}$ & $80 \mathrm{~N} \cdot \mathrm{m} / \mathrm{rad}$ \\
\hline $\mathrm{K}_{\text {min }}^{\circ}$ & $1 \mathrm{~N} \cdot \mathrm{m} / \mathrm{rad}$ \\
\hline$\theta_{\mathrm{hm}}$ & $3.67 \mathrm{rad}$ \\
\hline $\mathrm{I}_{\mathrm{hm}}$ & $0.25 \mathrm{~m}$ \\
\hline $\mathrm{b}$ & $250 \mathrm{~N} \cdot \mathrm{s} / \mathrm{m}$ \\
\hline $\mathrm{m}$ & $0.28 \mathrm{~kg}$ \\
\hline $\mathrm{L}$ & $0.33 \mathrm{~m}$ \\
\hline
\end{tabular}

Introducing the parameters from Table $6 \mathrm{~B}$, along with a maximum torque of $16 \mathrm{~N} \cdot \mathrm{m}$, into equation (4-82) and (4-79) and rearranging, the maximum force required from the springs is $\mathrm{F}_{\mathrm{max}}=168 \mathrm{~N}$.

\section{Configuration 2}

This configuration implements the same component arrangement as Configuration 1, but combines the inputs for control of stiffness and position. A diagram of the configuration is shown in Figure 6.6.

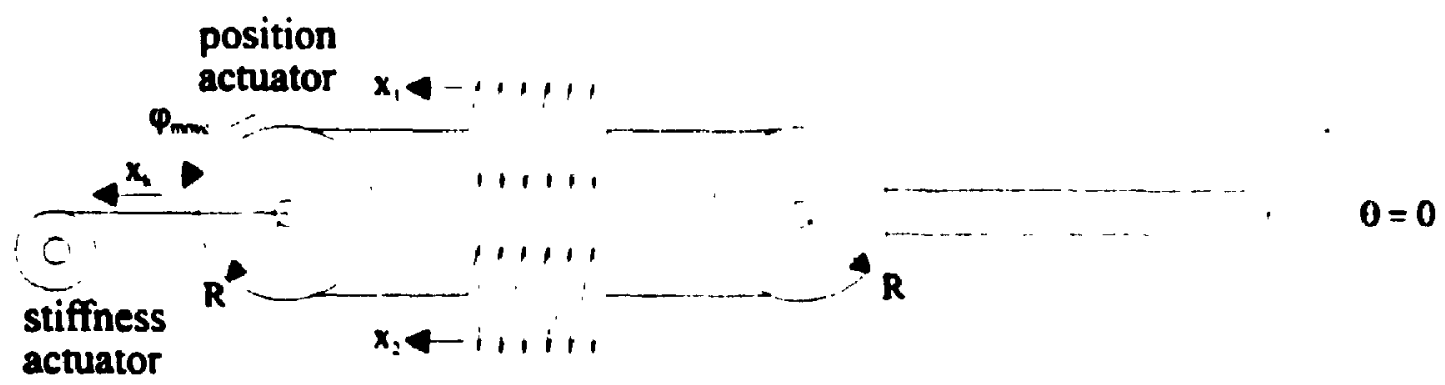

Figure 6.6. Configuration 2 (neglecting the dampers). 
Section 4.5.1 shows that this configuration reduces energy expenditure for motion of the arm. However, Section 4.2.1 shows that it has the same geometric and torque limitations as Configuration 1. Therefore the parameters in Table 6B and the same maximum spring force of $168 \mathrm{~N}$ apply.

\section{Configuration 3}

Section 4.5.4 (Figure 4.22) suggests the addition of a third spring to neutralize the spring forces necessary to set the joint stiffness. As well, Section 6.1 (Figure 6.3) suggests moving the position actuator of Configuration 2 between the springs and joint. This eliminates the need for space in which the springs can translate to move the joint. Spring length can then be increased, resulting in improved performance. As well, since the dampers are used to control the oscillation of the joint, they can be combined into one and connected to the spring end of the joint (see Figure 6.7). This also eliminates damper forces when changing the stiffness. A diagram of the configuration is shown in Figure 6.7.

In this configuration, $\theta_{\mathrm{hm}}$ is mathematically equivalent to zero (see Section 6.1). Therefore, equation (4-79) can be simplified to

$$
\alpha=\frac{1}{2 K_{\max }^{\circ}} \sqrt{8(1+\eta) \mathrm{F}_{\max }\left(\mathrm{K}_{\max }^{\bullet}-\mathrm{K}_{\min }^{\bullet}\right) \mathrm{l}_{\mathrm{um}}}
$$

Replacing $\alpha$ in equation (6-1) with equation (6-2) and rearranging,

$$
F_{\operatorname{mx}}=\frac{2 T_{\max }^{2}(1+\eta)\left(K_{\max }^{*}-K_{\min }^{*}\right)}{K_{\max }^{2} l_{\max }}
$$

For a maximum torque of $16 \mathrm{~N} \cdot \mathrm{m}$, and using the same parameter values of Table $6 \mathrm{~B}$ with the exception that $\theta_{\mathrm{hm}}=0$ and damping factor $B=40 \mathrm{~N} \cdot \mathrm{s} / \mathrm{m}$, the maximum force that must be produced by the springs is $F_{\max }=50.56 \mathrm{~N}$. Note that this configuration not only 
saves the most in terms of energy and power, but also requires the least spring force to achieve the same maximum torque.

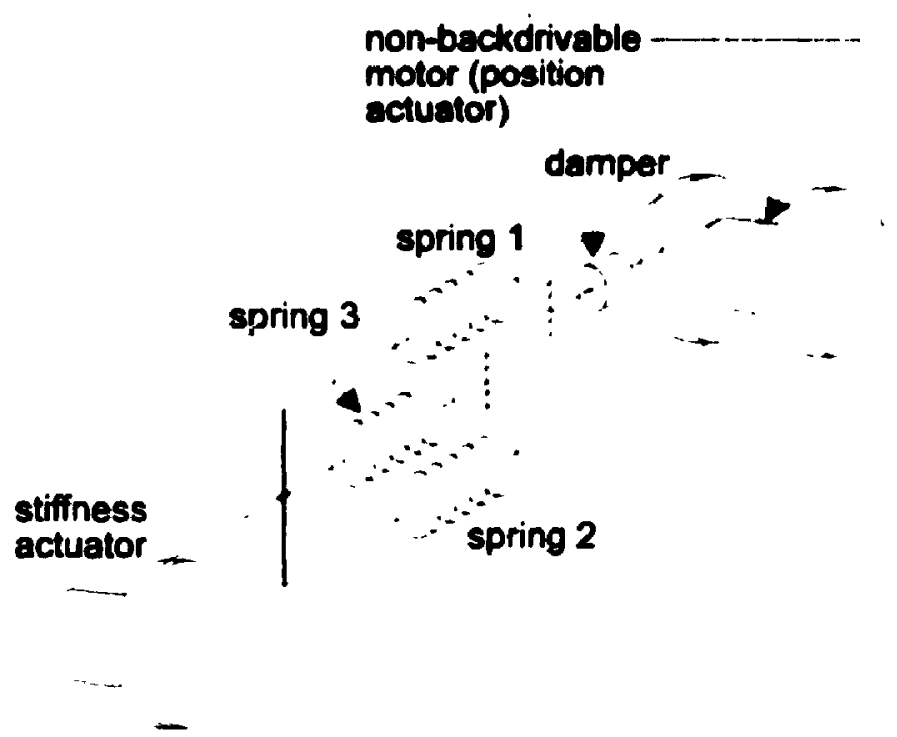

Figure 6 7: Configuration 3.

\section{Configuration 4}

This configuration is identical to Configuration 3, except for the fact that spring size and joint radius are kept identical to those in Configurations 1 and 2 . The selected parameters are identical to those used in Table 6B.

The state space representations of the models for each configuration are presented in Appendix B. 


\subsubsection{Task Definitions}

Comparison between configurations requires a measure of performance. Ideally, the configurations would be tested under normal daily operation. At this stage, however, it is important to predict the performance based on a variety of simulated tasks. There are four basic tasks that each configuration performs. Each is discussed below.

\section{Task 1}

The purpose of the design is to allow variable stiffness of the joint. Therefore, it is expected that the joint stiffness will be changed regularly during normal daily routines. For this task, the arm is initially at rest at minimum stiffness $(1 \mathrm{~N} \cdot \mathrm{m} / \mathrm{rad})$ in a gravity field. The equilibrium position is set to 0 degrees. Starting at $t=0$ seconds, the joint stiffness is increased from minimum to maximum ( $80 \mathrm{~N} \cdot \mathrm{m} / \mathrm{rad})$. The change in stiffness occurs over a 0.5 second period, and the spring stretch is commanded to occur with an acceleration and deceleration of $100 \mathrm{~m} / \mathrm{s}^{2}$. Note that these accelerations are sommanded to avoid discontinuities associated with instantaneous velocities in the simulation.

\section{Task 2}

To distinguish dynamic effects from gravity effects, Task 1 is repeated in a horizontal plane, i.e., the gravity is removed.

\section{Task 3}

Robotic manipulators tend to be designed for contact tasks. There are exceptions, of course, which include welding and spray painting robots in manufacturing. However, most of these manipulators, including prostheses, perform unconstrained motion for much of their regular operation. It is therefore important to simulate the performance of the variable stiffness joint in free motion. The joint stiffness is set at $50 \mathrm{~N} \cdot \mathrm{m} / \mathrm{rad}$. The 
equilibrium position is initially set at -5 degrees in a horizontal plane (no gravity). The arm is commanded to move 10 degrees upward to +5 degrees starting at $t=0$ seconds. The movement occurs over a 0.5 second period, and the commanded acceleration and deceleration of the arm are $100 \mathrm{~m} / \mathrm{s}^{2}$. Again, these accelerations are commanded to avoid discontinuities in the simulation. The actual acceleration and deceleration of the arm are dictated by the forces created within the model.

\section{Task 4}

As mentioned above, most manipulators are designed to perform contact tasks. This is certainly true in the case of a prosthesis since humans regularly interact with their environment. In fact, the purpose behind the design of the variable stiffness joint is to improve performance in contact while maintaining good performance for unconstrained motion as well. It is therefore important to simulate the execution of a contact task. So as not to confuse issues, this task is not performed in a gravity field. Once again, this corresponds to a movement of the arm in the horizontal plane. Joint deflections and oscillations are therefore a result of contact only and do not include gravity effects. The task is identical to that commanded in Task 3 . However, when the arm ieaches 0 degrees, it comes in contact with an effectively rigid constraint. The stiffness of the constraint increases linearly from 0 to $10,000 \mathrm{~N} \cdot \mathrm{m}$ within the first $0.5 \mathrm{~mm}$ of the surface to avoid numerical problems in the simulation associated with discontinuities. Beyond $0.5 \mathrm{~mm}$ the constraint is modelled as having a constant stiffness of $10,000 \mathrm{~N} \cdot \mathrm{m}$. The acceleration, deceleration, and period of commanded motion of the equilibrium position are identical to those of Task 3.

Proper judgment of performance requires analysis of relevant variables. The inputs for each task are plotted to view exactly what motion is commanded. For 
Configuration 1, the inputs are $x_{1}$ and $x_{2}$. The inputs for the other configurations are $x_{k}$ and $x_{\text {move }}$ The commanded equilibrium position and resulting actual position of the joint are also plotted for a visual indication of the arm position and endpoint deflection. The resulting spring stretches, joint stiffiness, springs and damper forces, and joint torque are also plotted as an indication of the internal joint properties. The joint torque that is plotted is the sum of the effects of the system components. This is not the same as the external torque except in static equilibrium. The difference between the two is the net torque that is applied to the inertia. In addition, the torque due only to spring forces is plotted to distinguish the effects of springs and dampers. The energy and power required for each task are also plotted as a measure of performance and an indication of the power and energy required from the actuators. The results of the simulations are shown in Figures 6.8 to 6.23 .

The simulations were performed on MATLAB. The MATLAB code for each task and configuration are given in Appendix $C$. 


\subsubsection{Simulation Results}

\section{(a) Task 1}

The joint stiffiness increases from $1 \mathrm{~N} \cdot \mathrm{m} / \mathrm{rad}$ to $80 \mathrm{~N} \cdot \mathrm{m} / \mathrm{rad}$ in 0.5 seconds in a gravity field.

\section{Confirumtion 1}

The results for this configuration are shown in Figure 6.8. The inputs $x_{1}$ and $x_{2}$ are : creased identically (Figure 6.8(a)). This correctly corresponds to the mode through which stiffness is changed for this configuration. Although it is not apparent from the plot, there is an $100 \mathrm{~m} / \mathrm{s}^{2}$ acceleration and deceleration of the inputs. Since these are quite high values it appears to instantly accelerate and decelerate to a constant velocity of the inputs. In (b) it can be seen that the arm is initially deflected to approximately -23 degrees. This is due to the weight of the arm in gravity and the low (minimum) stiffness. As the stiffness increases, the deflection of the arm due to the weight decreases. Notice that the equilibrium position of the joint does not change.

The stretch of the springs in (c) warrants some explanations. The arm is initially set at low stiffness so the springs should be unstretched $\left(\delta_{1}=\delta_{2}=0\right)$. Hiowever, the joint is deflected due to the weight of the a.m. This causes spring 1 to be initially stretched while spring 2 is compressed by the some amount. When the joint stiffness is increased, the stretch of both springs increases slightly in the positive direction. The effect of the changing stiffness on joint deflection becomes more prominent at that point. Spring 1 decreases slightly in length while spring 2 increases very quickly due to the combined effects of smaller joint displacement and increasing stiffness. At approximately 0.2 seconds, the change in joint displacement slows (Figure 6.8(b)), and the effect on spring stretch is almost entirely due to the increasing stiffness. The springs finally come to rest at approximately the same value, with the difference due only to the slight joint 
displacement due to the weight of the arm at high stiffness. The corresponding change in joint stifiness is shown in (d).

The quick change of velocity due to the high acceleration and deceleration can be seen in the damper forces in (e). The quick change of spring 2 deflection is seen as an increased damper force, while the reversing deflection of spring 1 causes the force in damper 1 to dip into the negative direction. Spring forces generally follow from the spring stretches in (c), however the decrease in force for increasing displacement requires some explanation. Note from Figure 4.3 that the peak negative force does not occur at the maximum negative displacement. The spring force actually begins to increase as the spring is negatively displaced beyond this peak. Thus the initial deflection of spring 2 is beyond this peak. Positively displacing the spring causes the force to initially decrease to the peak negative value and then increase to a positive value. This effect is not the same in spring 1. The displacement is initially positive and thus on the positive side of the peak. The decreasing force in spring 1 corresponds to the period in which the spring decreases in deflection (Figure 6.8(c)).

The effect of the component forces can be seen in (f). The initial torque is due to the deflection caused by the weight of the arm. The difference in spring forces causes a large torque as the stiffness increases. However, the forces created in the dampers counteracts this large force and reduces the net torque to less than $1 \mathrm{~N} \cdot \mathrm{m}$. Without the dampers, this excess spring toque would have been applied to the inertia of the arm, causing it to oscillate.

The energy and power requirements are presented in (g) and (f). There are no requirements before approximately 0.35 seconds. This is due to the fact that the net force of each branch is negative, i.e., compressive. The actuators are changing the branch inputs in the same direction as the branch forces, thus no external energy or power is required. However, at approximately 0.35 seconds the net branch forces become positive and the actuators have to pull against them. The total energy expended during the task is 
approximately 2.3 Joules and each motor needs a maximum of approximately $14 \mathrm{~W}$ of power.

\section{Confiruration 2}

The results for this configuration are shown in Figure 6.9. Note from $(a)$ that the inputs are different from those of Figure 6.8(a). In Configuration 2, the inputs are separated into $x_{k}$ and $x_{\text {move }}$. For this task, $x_{k}$ increases in order to raise the joint stiffness.

Note that $\mathbf{x}_{\text {move }}$ does not change, corresponding to the constant equilibrium position in (b). Plots (b) to (f) in Figure 6.9 are identical to those in Figure 6.8. This is due to the identical nature of the components between the two configurations. The difference lies only in the inputs. This difference can be seen in the energy and power requirements of (g) and (n). The total energy consumed is identical to that of Configuration 1 (Figure 6.8(g)). However, in this case all of the energy is used by the stiffness actuator. No energy is needed by the position actuator since no position change is commanded. The same is true for the power requirements. The same total power required is the same, but it is required by one actuator rather than two. This means that an actuator capable of approximately $28 \mathrm{~W}$ output is required instead of two $14 \mathrm{~W}$ actuators. The better of the two situations dep..ds upon the application and criteria of the designer.

\section{Configuration 3}

The results for this configuration are shown in Figure 6.10. Note that the plots for Figures 6.10(a) to (f) are the same shape as in Figure 6.9. However, the magnitude of displacements and forces have changed. In fact, the inputs and spring displacements have increased by a factor of approximately 3 while the forces have decreased by the same factor. The tradeoff between force and space in the springs is made apparent in this case (forces can be decreased by increasing the spring length and vice versa). Note, however, that although the springs are larger in this configuration, the total size of the 
system has not increased. The space occupied by the larger springs is created by eliminating the need for translation of the components for an equilibrium position change. The resulting internal forces are reduced, as seen in (e). From an external view though, i.e., from plots (b), (d), and (f), nothing has changed. Figures $6.10(\mathrm{~g})$ and (h) show reduced energy and power consumption. This is not due to the change of the position actuator, but rather the addition of the third spring. In this case, the negative forces of the branch springs due to the initial stiffness setting are cancelled out by the same force created in the third spring. Thus the forces on the stiffness actuator are directly due to the external loading of the joint. The energy and power consumption thus begin immediately upon initialization of the task, but values are approximately half of the previous configurations due to the r luced net force on the stiffness actuator. The total energy consumed is approximately $1.1 \mathrm{~J}$ while power requirements peak at about $14 \mathrm{~W}$. In addition, as the endpoint deflection approaches zero (Figure 6.10(b)), the net difference in force between the sum of the branch springs and the third spring approaches zero. Thus only negligible energy and power are required to further increase the stiffness.

\section{Configuration 4}

The results for this configuration are shown in Figure 6.11. The plots (a) to (f) are identical to those in Figure 6.9 (and 6.8 excluding the inputs). This is identical to Configuration 3 except that the springs are sized identically to the other configurations. Thus the forces and deflections correspond to the first two results. Since the springs are the same size, the entire system takes up much less space than in the previous configurations. Again this shows the tradeoff between internal forces and space requirements. Notice that the resulting energy and power consumption are identical to those of Figure 6.10. This shows that changes in spring length and force do not affect any external results. The force and length are simply chariged by $a$ lever ratio. 

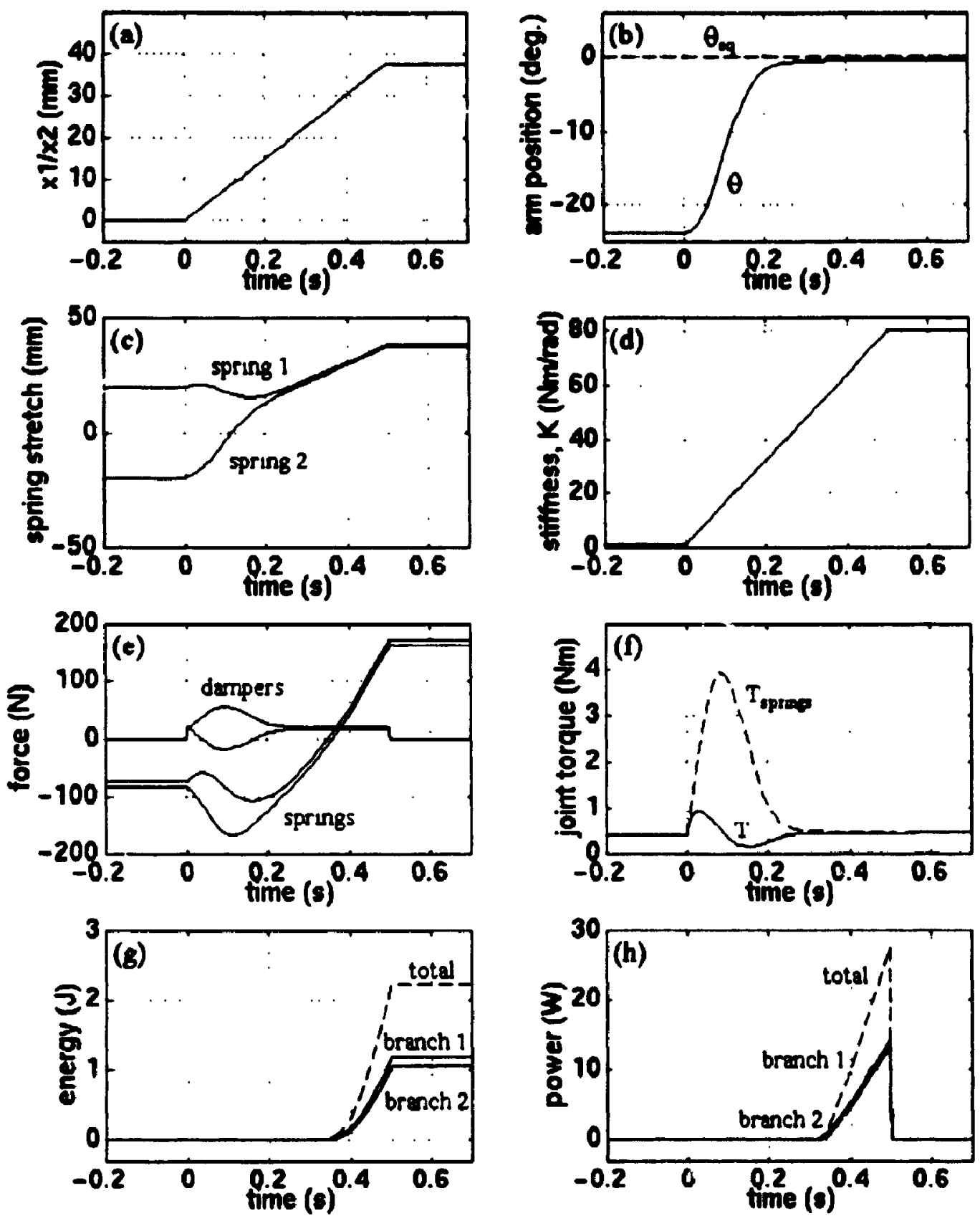

Figure 6.8: Task 1, Configuration 1 results, (a) $x_{1}$ and $x_{2}$, (b) $\theta_{\infty}$ and $\theta$, (c) $\delta_{1}$ and $\delta_{2}$, (d) $K^{*}$, (e) spring and damper forces, (f) net joint torque and joint torque due to springs only, (g) actuator output energy expenditure, (h) required actuator power 

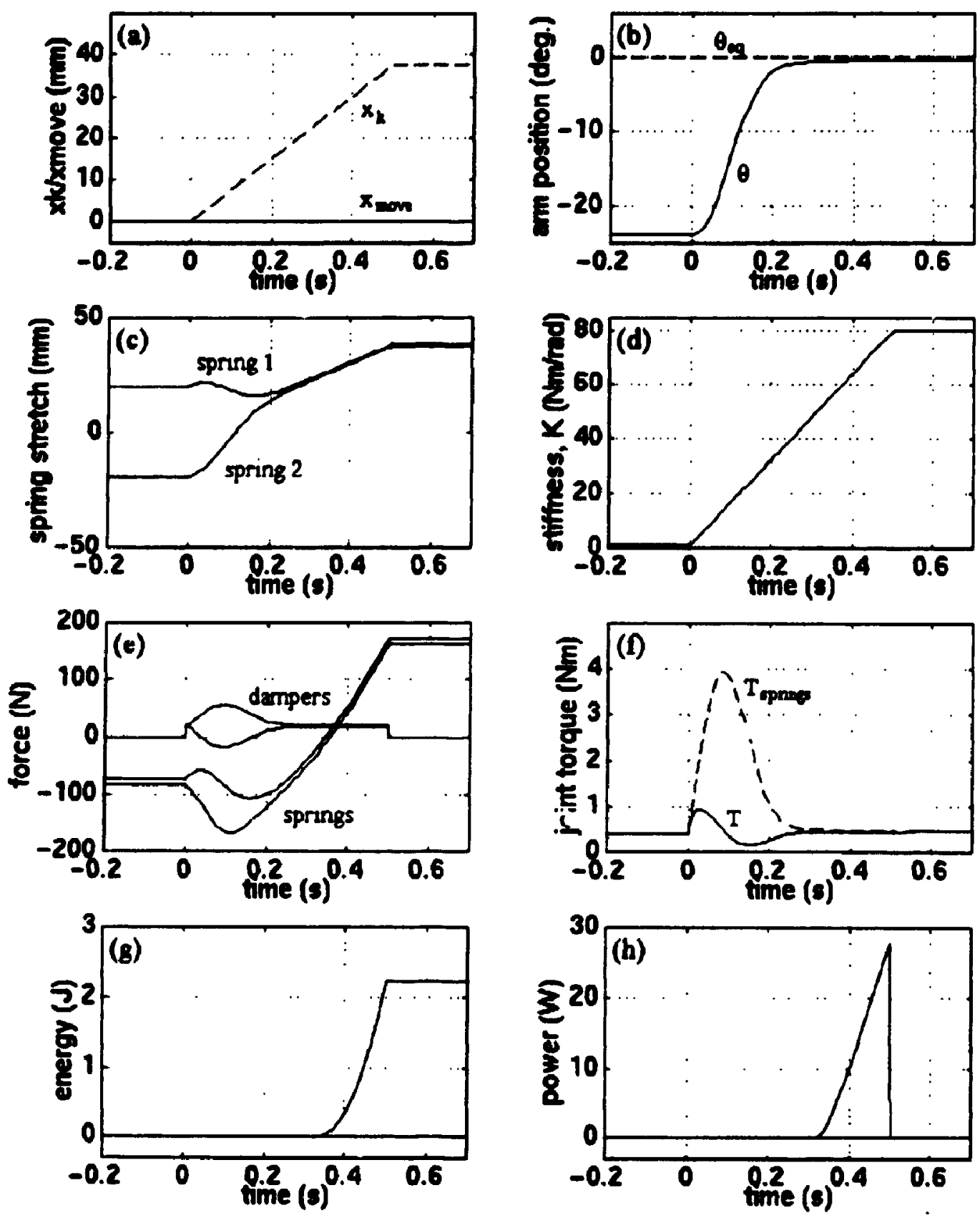

Figure 6.9: Task 1, Configuration 2 results, (a) $x_{k}$ and $x_{\text {move }}$ (b) $\theta_{e q}$ and $\theta$, (c) $\delta_{1}$ and $\delta_{2}$, (d) $K^{*}$, (e) spring and damper forces, (f) net joint torque and joint torque due to springs only, (B) actuator output energy expenditure, (h) required actuator power. 

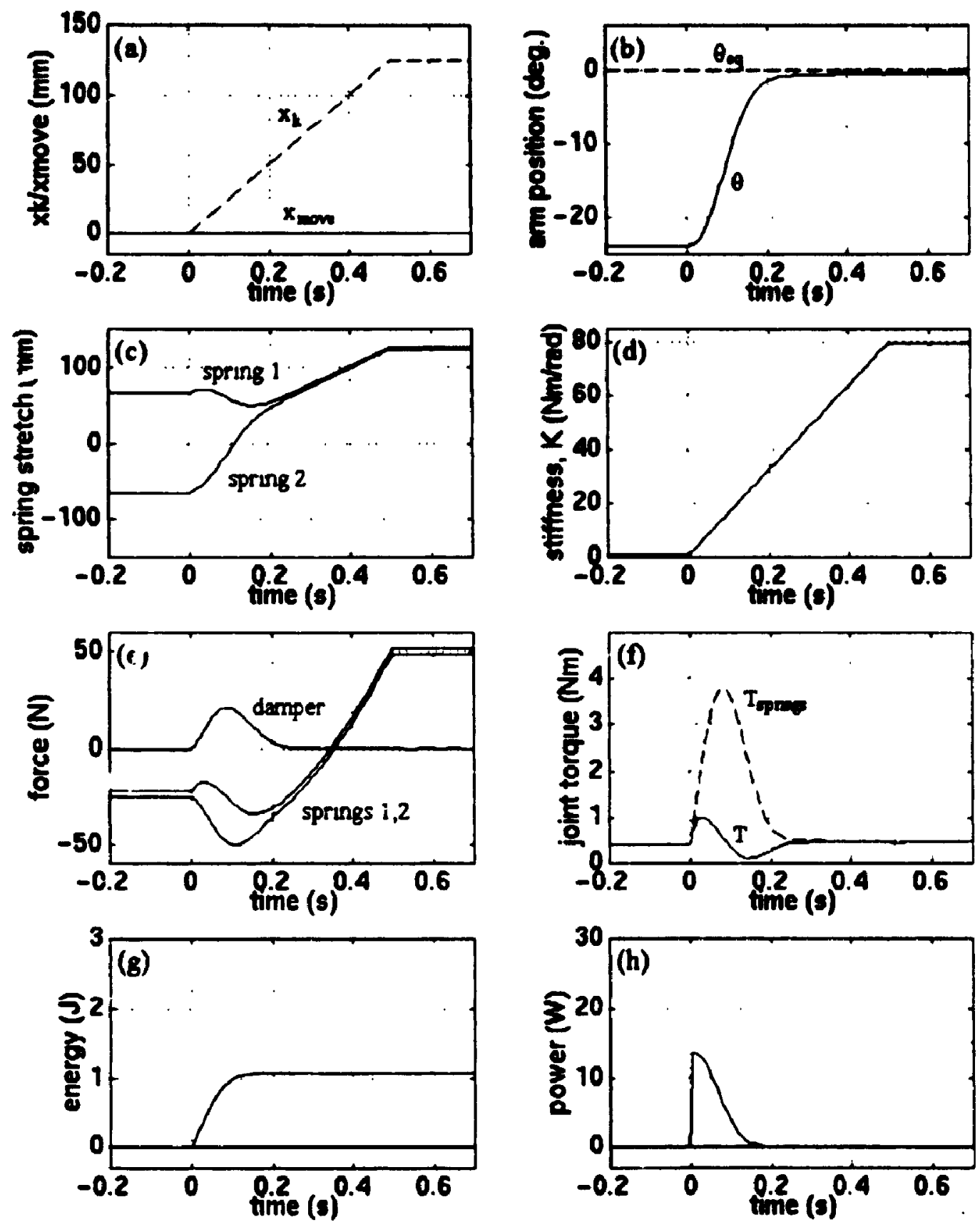

Figure 6. 10: Task 1, Configuration 3 results, (a) $x_{k}$ and $x_{\text {mone }}$ (b) $\theta_{e q}$ and $\theta$, (c) $\delta_{1}$ and $\delta_{2}$, (d) $k^{*}$. (e) spring and damper forces, (f) net joint torque and joint torque due to springs only, (B) actuator output energy expenditure, (h) required actuator power. 

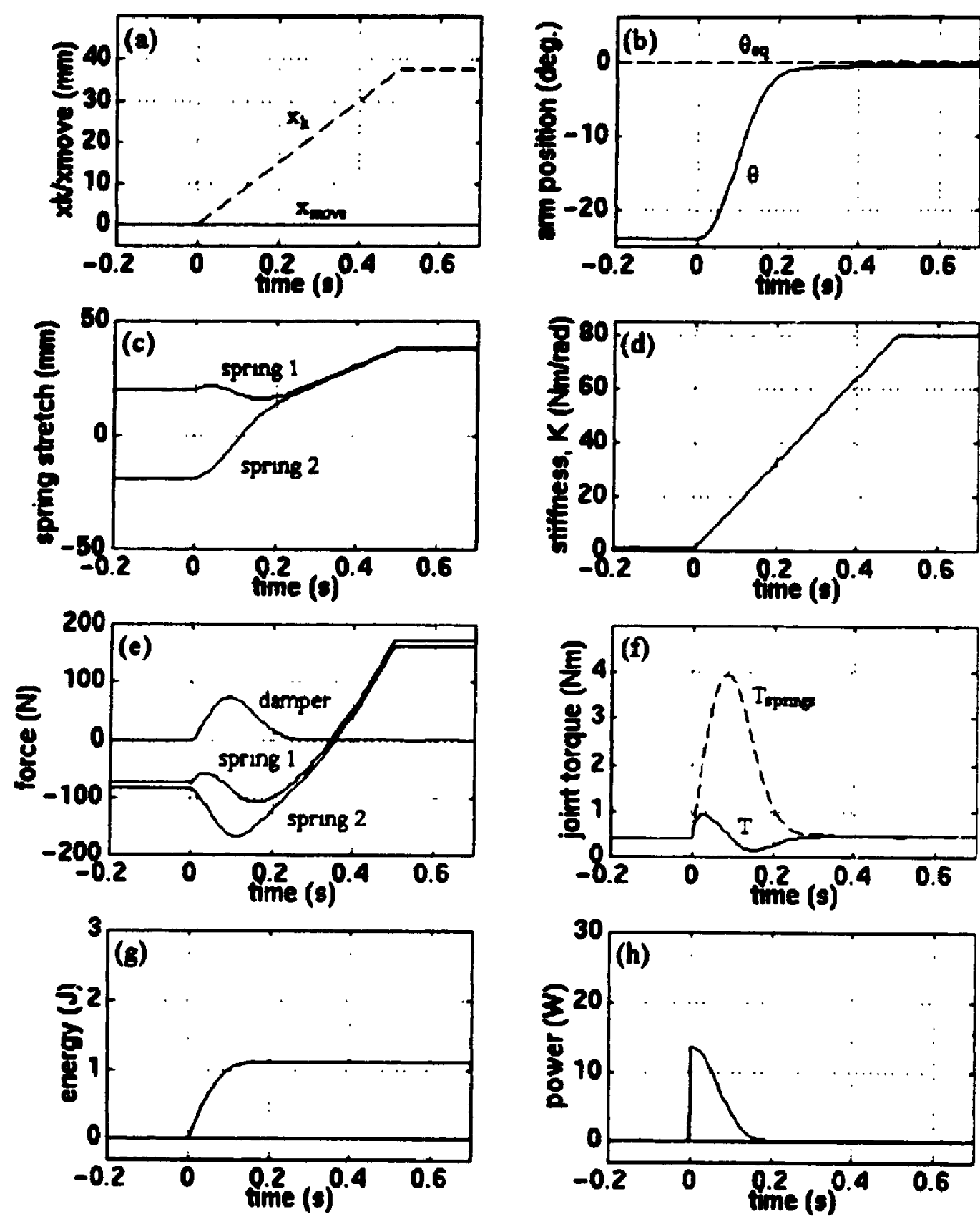

Figure 6.11: Task 1, Configuration 4 results, (a) $x_{k}$ and $x_{\text {move }}$ (b) $\theta_{c q}$ and $\theta$, (c) $\delta_{1}$ and $\delta_{2}$, (d) $k^{*}$, (e) spring and damper forces, (f) net joint torque and joint torque due to springs only, (g) actuator output energy expenditure, (h) required actuator power. 


\section{(b) Task 2}

The joint stiffness increases from $1 \mathrm{~N} \cdot \mathrm{m} / \mathrm{rad}$ to $80 \mathrm{~N} \cdot \mathrm{m} / \mathrm{rad}$ in 0.5 seconds without gravity.

\section{Configuration 1}

The results for this configuration are shown in Figure 6.12. The inputs in (a) are identical to those of Task 1 (Figure 6.8(a)). This is because the tasks are the same from the controller point of view. The difference is the absence of gravity from this task. Without gravity, the weight of the arm does not cause a joint displacement from the equilibrium position as shown in (b). The system is therefore symmetrical so the individual spring displacements and forces, and damper forces, are identical for the two branches. The energy and power consumption in (g) and (h), respectively, are identical to those of Task 1, implying that the increased potential energy associated with the lifting of the arm in a gravity field comes entirely from the springs.

\section{Configuration 2}

The results for this configura..on are shown in Figure 6.13. The same analysis of Configuration 1 for this task applies by analogy. The inputs are identical as in Task 1 , the symmetry is evident from the displacements and forces, and the power and energy consumption changes are negligible.

\section{Confopuration 3}

The results for this configuration are shown in Figure 6.14. The same analysis applies to this configuration as the previous two. Note that since there is no longer a displacement of the joint (Figure 6.14(b)), the net difference in force between the sum of the branch springs and the third spring is eliminated. This means that the stiffness actuator can change the stiffness with no forces placed on it, and thus no energy or power 
requirements. This confirms the suggested improvement of the configuration in Section 4.5.4.

\section{Confiruration 4}

The results for this configuration are shown in Figure 6.15. The same comparison between tasks applies as in the other configurations. As in Configuration 3, there are no power or energy requirements to change the stiffness. 

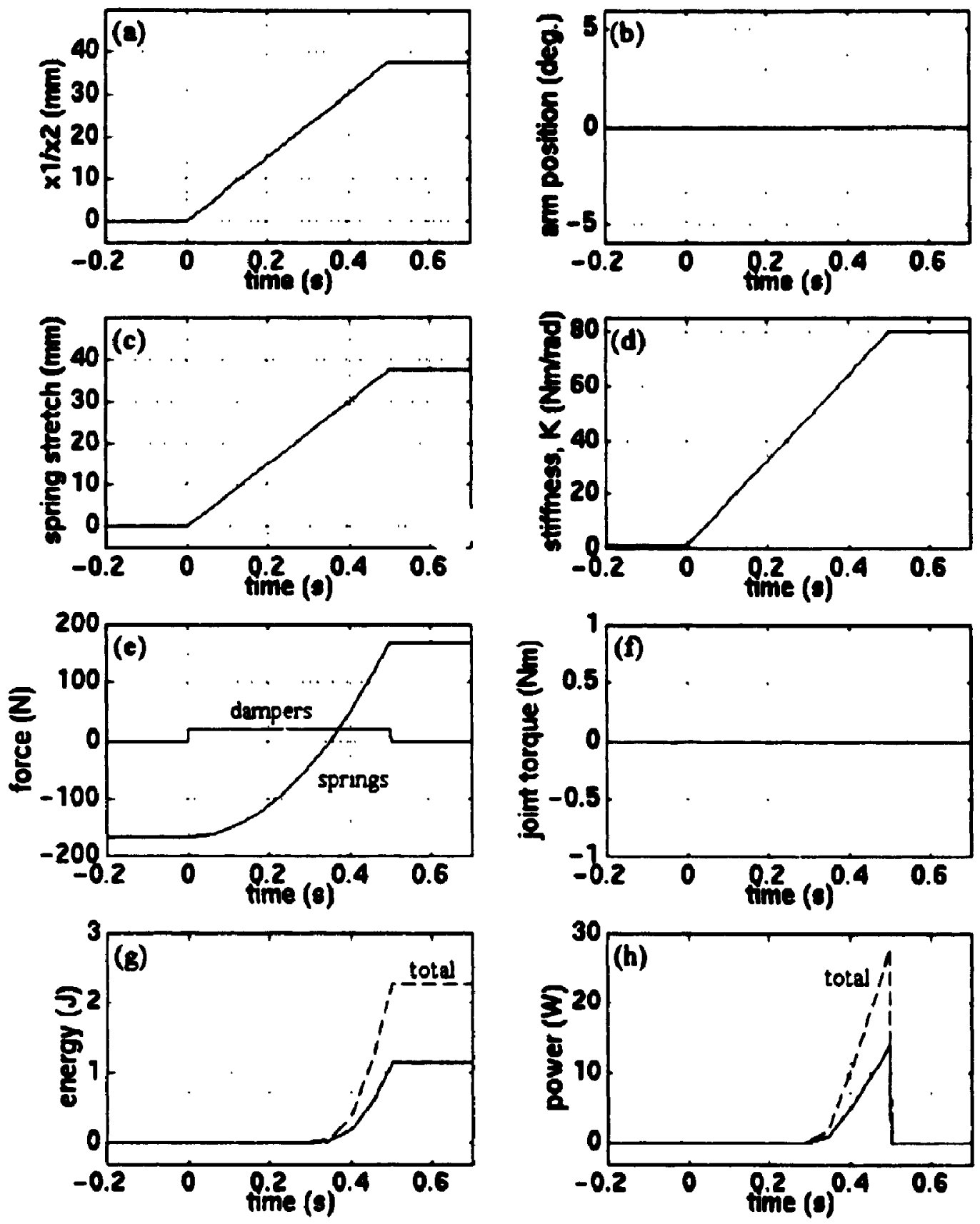

Figure 6.12. Task 2, Configuration 1 results, (a) $x_{1}$ and $x_{2}$, (b) $\theta_{\infty}$ and $\theta_{1}$ (c) $\delta_{1}$ and $\delta_{2}$, (d) $K^{*}$, (e) spring and damper forces, (f) net joint torque and joint torque due to springs only, (g) actuator output energy expenditure, (h) required actuator power 

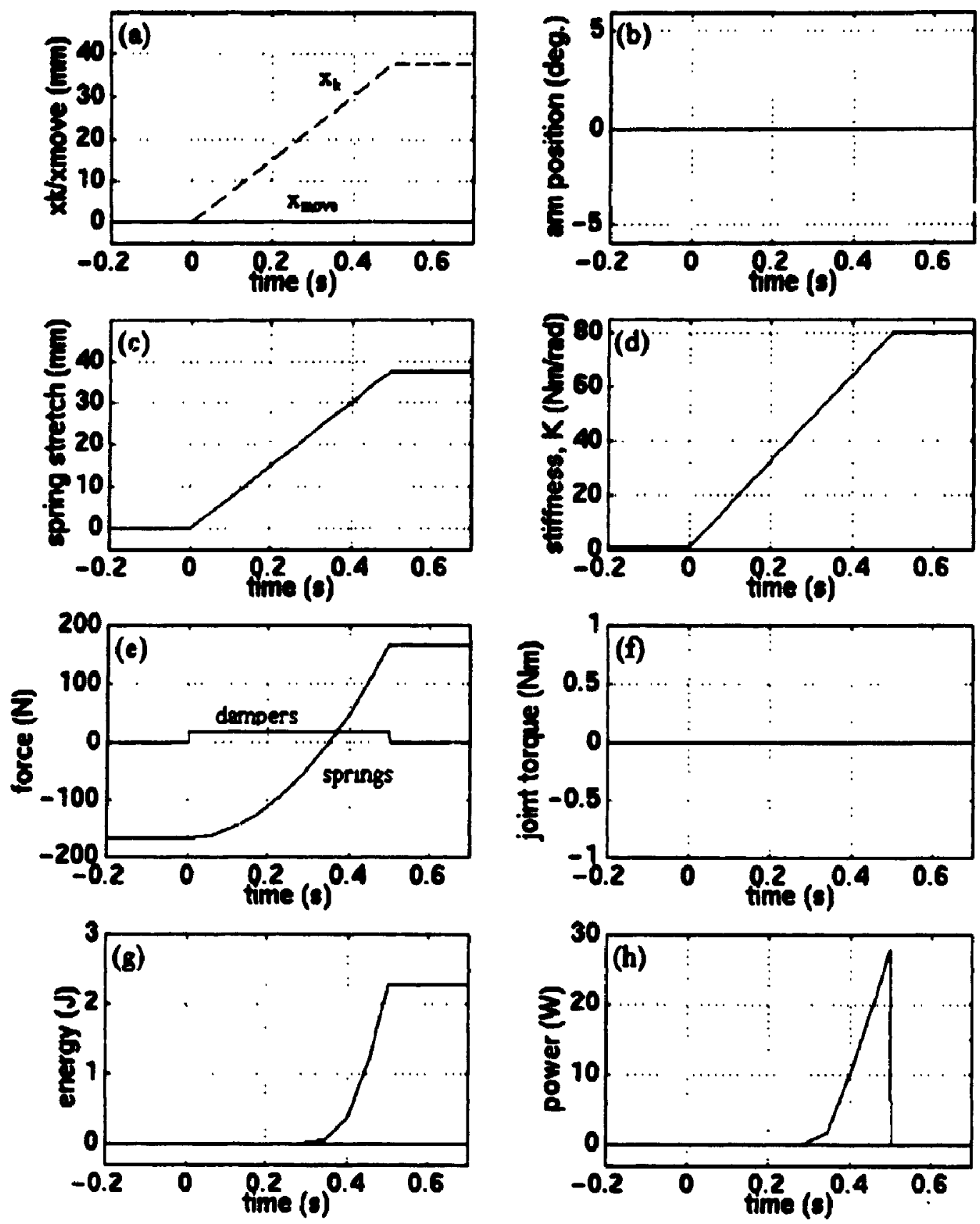

Figure 6.13. Task 2. Configuration 2 results, (a) $x_{k}$ and $x_{\text {move }}$ (b) $\theta_{\alpha q}$ and $\theta$, (c) $\delta_{1}$ and $\delta_{2}$, (d) $K^{*}$, (e) spring and damper forces, (f) net joint torque and joint torque due to springs only, (g) actuator output energy expenditure, (h) required actuator power. 

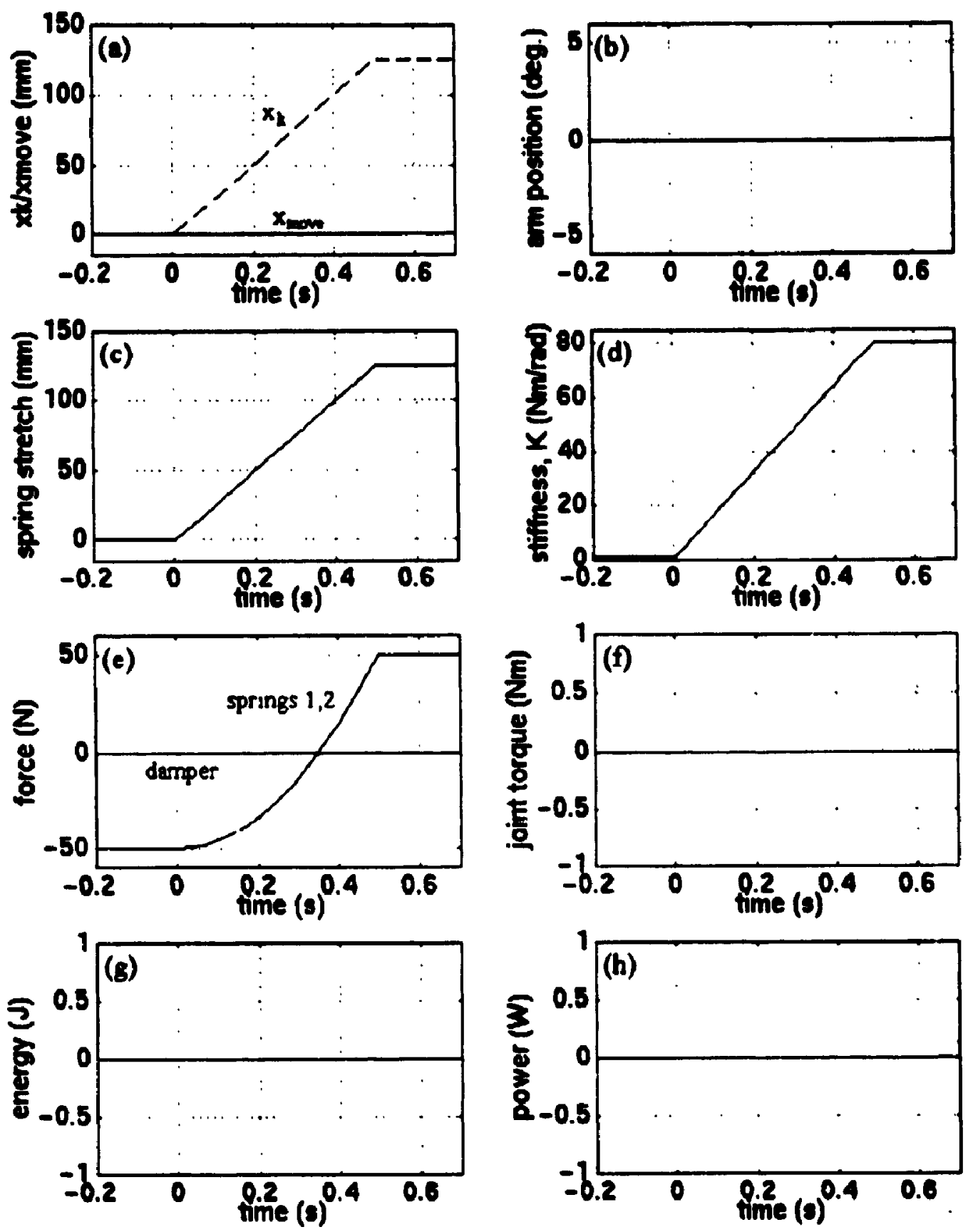

Figure 6. 14. Task 2, Configuration 3 results, (a) $x_{k}$ and $x_{\text {move }}$ (b) $\theta_{\infty q}$ and $\theta$, (c) $\delta_{1}$ and $\delta_{2}$, (d) $k^{*}$, (e) spring and damper forces, (f) net joint torque and joint torque due to springs only,

(g) actuator output energy expenditure, (h) required actuator power 

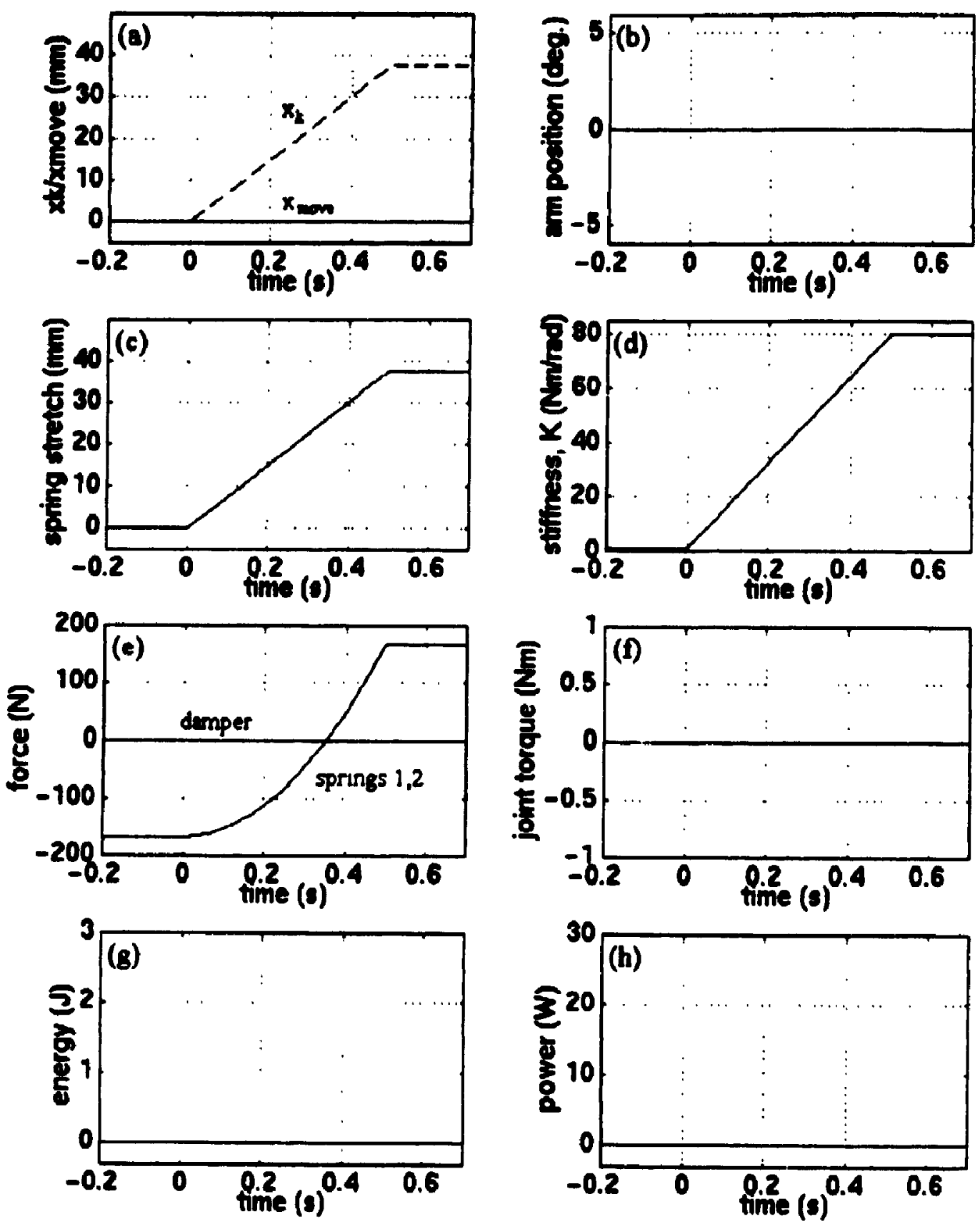

Figure 6.15: Task 2, Configuration 4 results, (a) $x_{k}$ and $x_{\text {move }}$, (b) $\theta_{\infty q}$ and $\theta$, (c) $\delta_{1}$ and $\delta_{2}$, (d) $k^{*}$, (e) spring and damper forces, $(f)$ net joint torque and joint torque due to springs only, (g) actuator output energy expenditure, (h) required actuator power. 


\section{(c) Task 3}

The arm is commanded to move from -5 degrees to +5 degrees in 0.5 seconds without gravity and at a joint stiffness of $50 \mathrm{~N} \cdot \mathrm{m} / \mathrm{rad}$.

\section{Confreuration 1}

The results for this configuration are shown in Figure 6.16. Initially the equilibrium position is at -5 degrees so the inputs $x_{1}$ and $x_{2}$ in (a) are offset from a zero position by the same amount in opposite directions. A motion is commanded in this task. Thus the $x_{1}$ and $x_{2}$ are moved in opposite directions by the same amount. As in Task 1 . there is an acceleration and deceleration of the inputs. However, this effect is not apparent in the plots due to their high value. The commanded position changes in (b) corresponding to the task definition. The actual position of the arm shows a slight initial lag when the arm is commanded to move due to the inertia of the arm. This same effect is seen when the motion is commanded to stop and the arm slightly overshoots its goal before settling to the equilibrium position. This phenomenon can also be seen from the individual sprih ${ }^{\circ} s$ stretches in (c). Note that the joint stiffness in (d) remains constant through the entire task, including these inertial displacements. This shows the decoupling of the joint stiffness from the endpoint deflection. The same inertial effects are seen in the spring and damper forces in (e) and joint torque in (f). Figure 6.16(g) shows that energy is consumed for pure motion, approximately $0.31 \mathrm{~J}$, as suggested in Section 4.5.1. This is also seen in the constant power requirement in (h). The initial peak power of about $0.7 \mathrm{~W}$ is the power required to overcome the inertia of the arm.

\section{Confiquration 2}

The results for this configuration are shown in Figure 6.17. The commanded position change is apparent by the plot of $x_{\text {move }}$ in (a). Since there is no stiffness change, $x_{k}$ remains constant. The system performance is identical to that of Configuration 1 
except for the energy and power requirements. The energy required for the motion, approximately $0.0014 \mathrm{~J}$, is only a fraction of that required for Configuration 1 . The initial power peak is still necessary to overcome the inertia of the arm. However, the peak is much lower at $0.12 \mathrm{~W}$ and there is no further power drain from the actuators.

\section{Confipuration 3}

The results for this configuration are shown in Figure 6.18. The results are again similar to those of Configuration 2, with the displacements and forces changed by the same factor as in Task 1. However, since there is no change in stiffness, the third spring does not cause any effects on energy or power consumption. Plots $(\mathrm{g})$ and $(\mathrm{h}$, in Figure 6.18 are identical to those for Configuration 2 in Figure 6.17.

\section{Configuration 4}

The results for this configuration are shown in Figure 6.19. Since forces and displacements are of the same magnitude, and the third spring is not used to chaige the stiffness, the results for this configuration ars identical to those of Configuration 2 (Figure 6.17). 

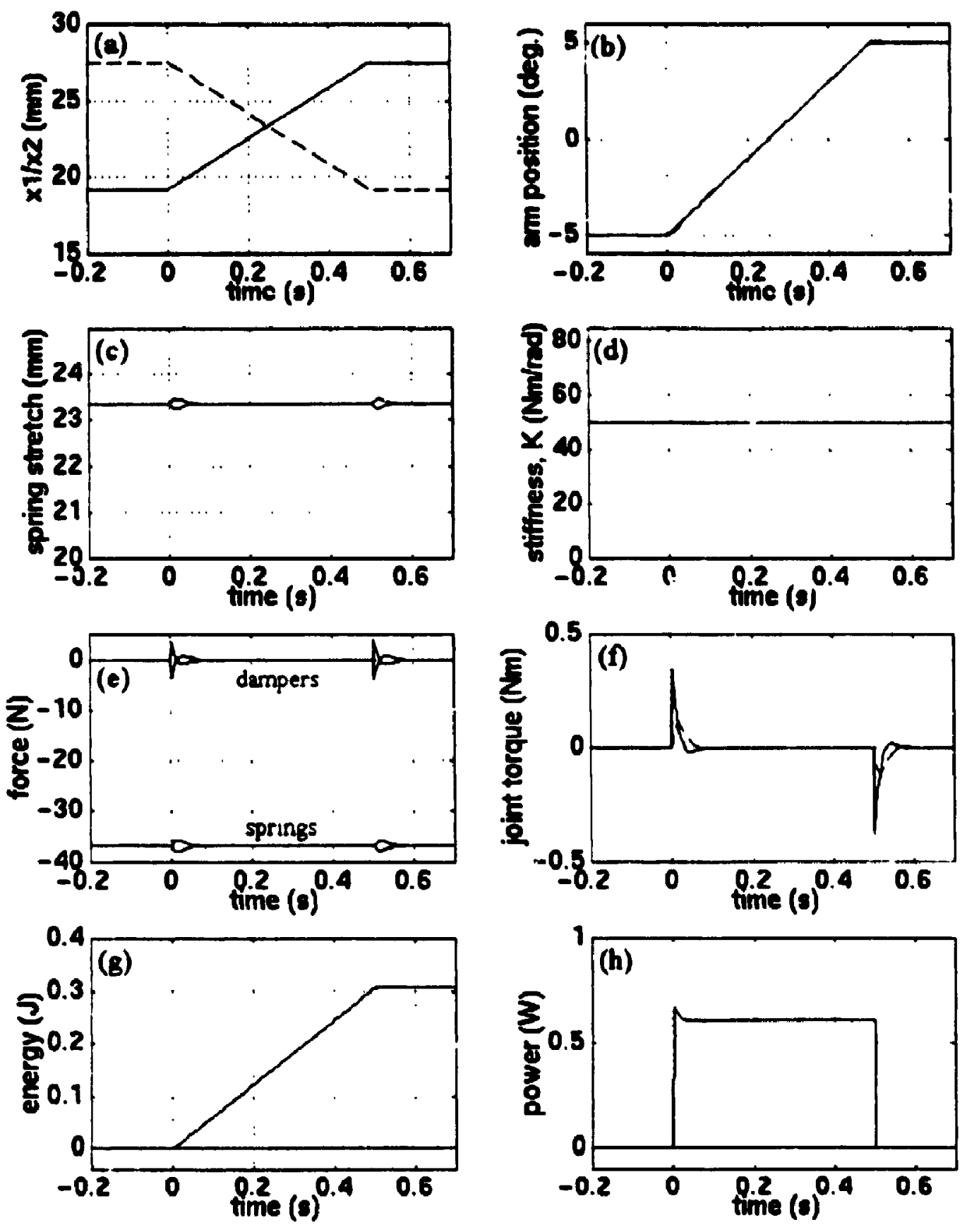

Figure 6.16: Task 3, Configuration 1 results, (a) $x_{1}$ and $x_{2},(b) \theta_{\infty q}$ and $\theta_{1}$ (c) $\delta_{1}$ and $\delta_{2}$, (d) $K^{*}$. (e) spring and damper forces, (f) net joint torque and joint torque due to springs only, (g) actuator ostput energy expenditure, (h) required actuator power 

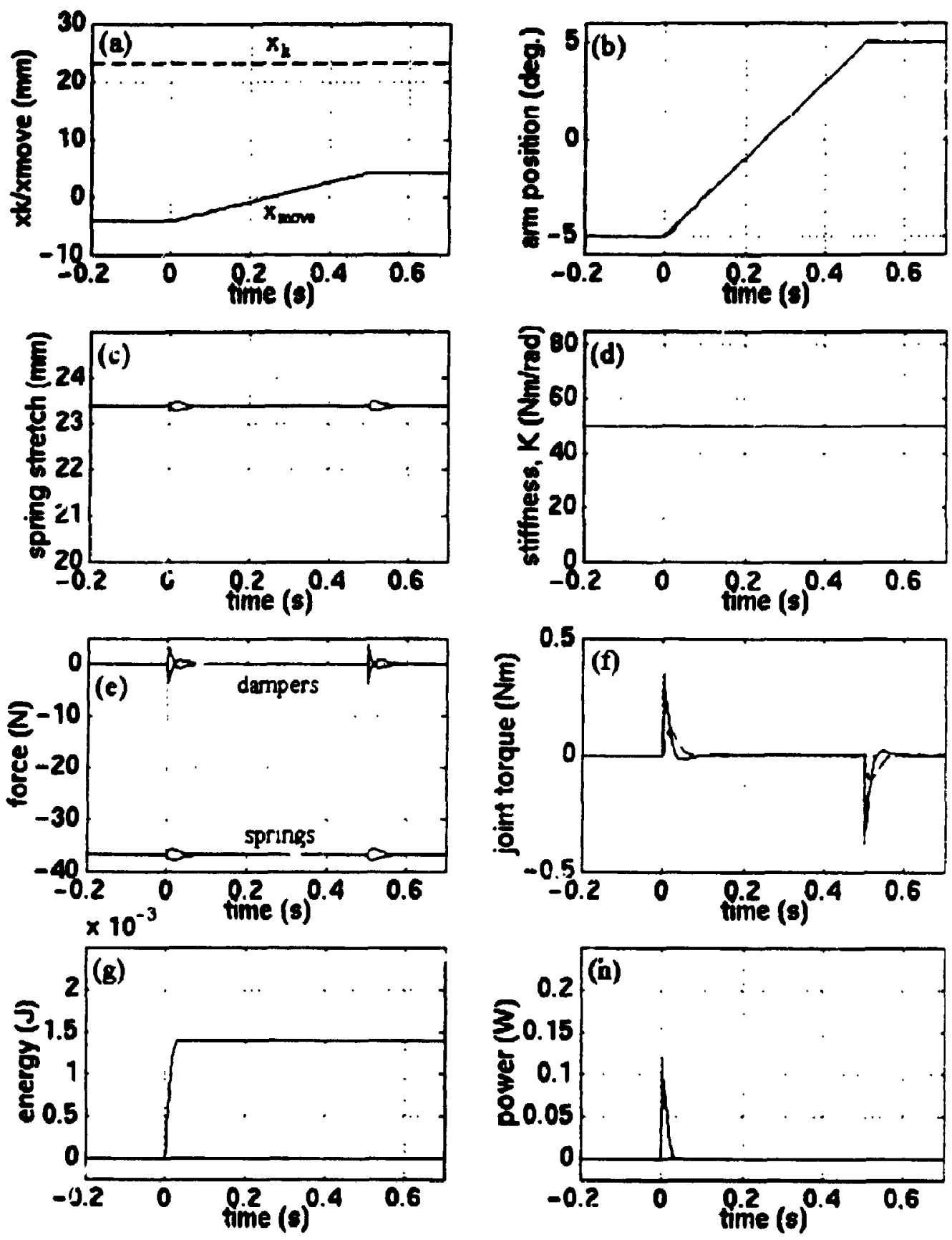

Figure 6.17: Task 3, Configuration 2 results, (a) $x_{k}$ and $x_{\text {move }}$ (b) $\theta_{e q}$ and $\theta_{1}$ (c) $\delta_{1}$ and $\delta_{2}$, (d) $K^{*}$, (e) spring and damper forces, (f) net joint torque and joint torque due to springs only,

(B) actuator output energy expenditure, (h) required actuator power. 

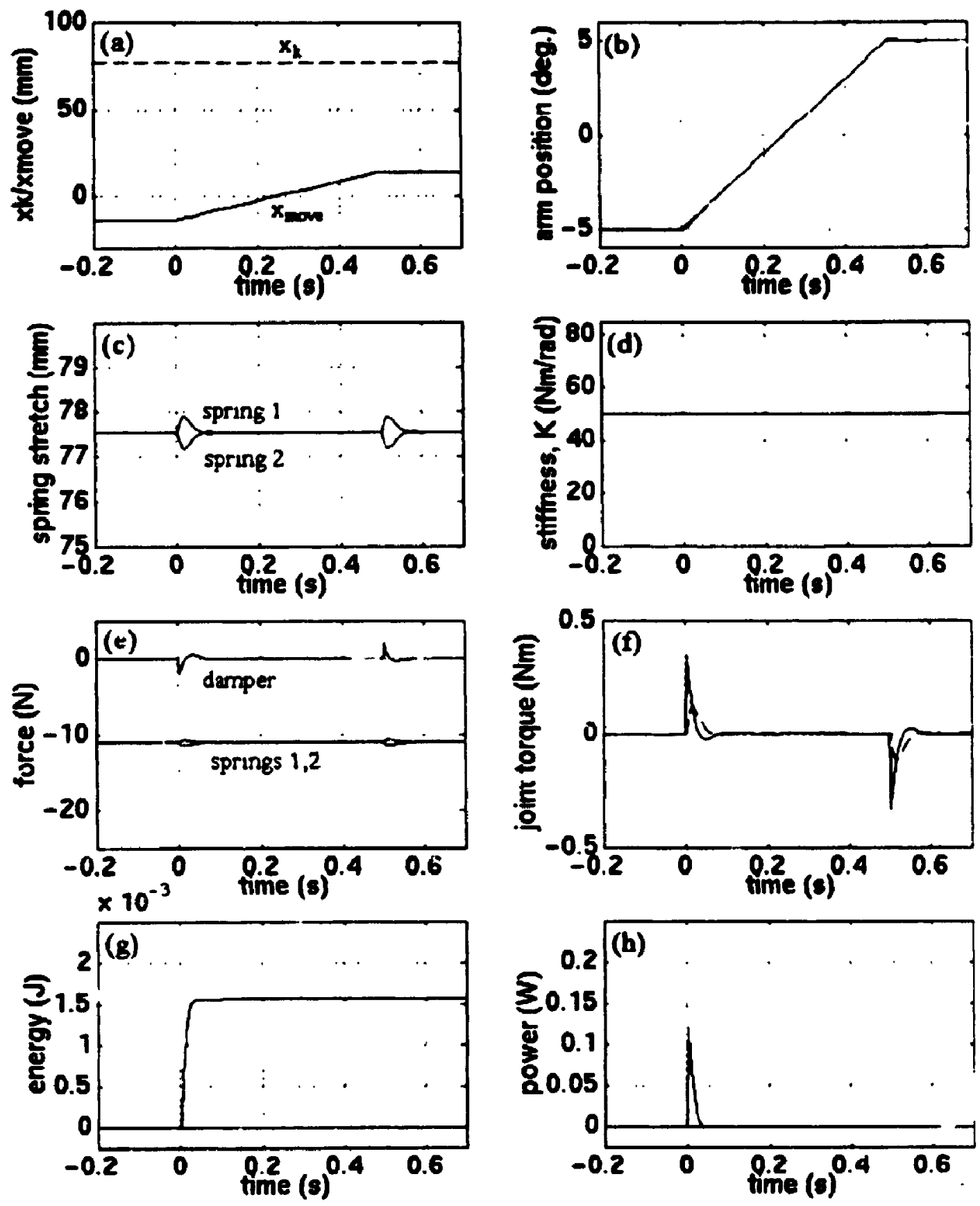

Figure 6.18: Task 3, Configuration 3 results, (a) $x_{k}$ and $x_{\text {move }}$ (b) $\theta_{\text {eq }}$ and $\theta$, (c) $\delta_{1}$ and $\delta_{2}$, (d) $K^{*}$, (e) spring and' tamper forces, (f) net joint torque and joint torque due to springs only, (g) a isator output energy expenditure, (h) required actuator power 

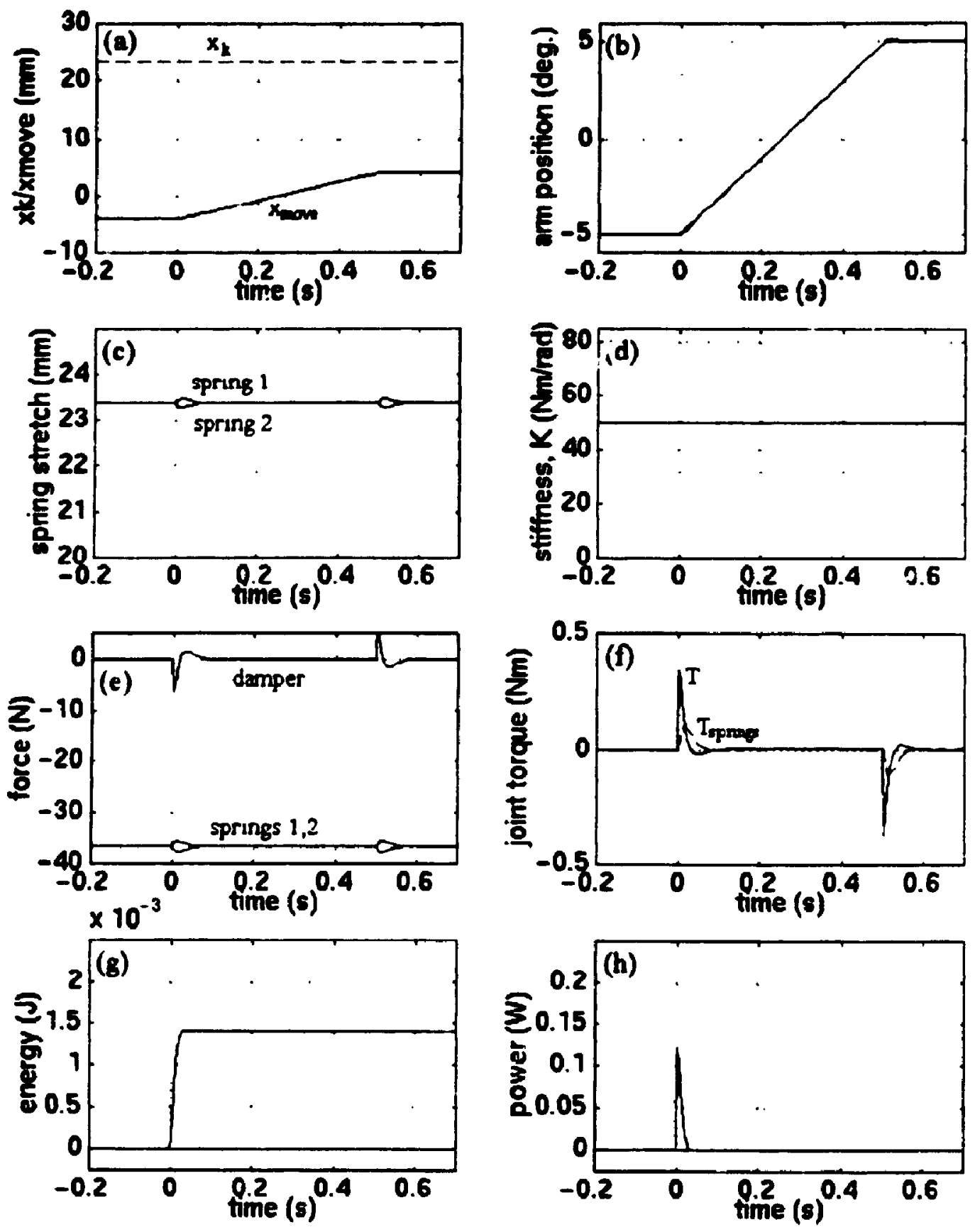

Figure 619 Task 3. Configuration 4 results, (a) $x_{k}$ and $x_{\text {move }}$, (b) $\theta_{\infty}$ and $\theta_{1}$ (c) $\delta_{1}$ and $\delta_{2}$, (d) $K^{*}$, (e) spring and damper forces. (f) net juint torque and joint torque due to springs only, (B) actuator output energy e: penditure, (h) required actuator power. 


\section{(d) Task 4}

As in Task 3, the arm is commanded to move from -5 degrees to +5 degrees in 0.5 seconds without gravity and at an initial joint stiffness of $50 \mathrm{~N} \cdot \mathrm{m} / \mathrm{rad}$. At 0 degrees it makes contact with a constraint having a stiffness of $10,000 \mathrm{~N} \cdot \mathrm{m}$, and joint stiffness is lowered to $1 \mathrm{~N} \cdot \mathrm{m} / \mathrm{rad}$ over the next 0.5 seconds.

\section{Conffeuration 1}

The results for this configuration are shown in Figure 6.20. This task combines a change in position, a change in stiffness, and contact with a constraint. The results are therefore representative of a general tash that would normally be required of the system. Initially the inputs are at some positive value. The average of the two corresponds to the portion of the input associated with spring stiffness. The difference between them is due to the location of the equilibrium position a! -5 degrees. As the equilibrium position approaches 0 degrees, the inputs reach the same value associated with the stiffness. At this point the inputs become the combined values of a position and stiffness change. The changing position causes the inputs to cross and become further apart. The general drop in average value is due to the decreasing stiffness. At 05 seconds, the commanded position change ceases and the inputs remain a constant distance apart. They continue to drop in value until the change in commanded stiffness stops at 0.75 seconds.

Figure 6.20 (b) shows the motion of the arm. There is an initial lag of the arm position at 0 seconds due to the inertia as in Task 3 . At 0.25 seconds, the arm makes contact with the constraint. The equilibrium position continues to +5 degrees as in Task 3. However, the arm is only able to push into the constraint a small distance due to the relatively high stiffness of the environment. There are two phenomena that affect the deflection of the constraint. First, as the commanded position continues into the surface, the deflection of the joint becomes greater and the joint torque increases. At the same time, the joint stiffness is decreasing which has the effect of reducing the joint torque 
Once the commanded position change ceases at 0.5 seconds, the arm position changes are due solely to the reducing stiffness. The stiffness change stops at 0.75 seconds and the arm and constraint settle to an equilibrium position.

The spring stretc hes in (c) appear similar in form to that of the inputs in (a). There are, however, differences in the plot as well as causalities. First, the springs are initially stretched to the same deflection. This corresponds to the stiffness setting. There are small changes in the deflections at $\mathbf{0}$ seconds due to the inertial effects of the arm beginning movement. These changes are not apparent on the plot due to the plot size. These fluctuations are only a fraction of a millimeter as in Task 3. At 0.25 seconds the deflections begin to drop due to the commanded stiffness reduction. However, the two springs separate in value due to the deflection of the joint. Note the difference in the cause of this separation from the similar one in (a). This separation also differs in that it is not linear. There is a slight curvature due to the varying deflection of the joint (see (b)). This is also true of the portion after 0.5 seconds where the stiffness continues to decrease. Note again in (c) that the stiffness change follows the task definition and is not affected by the deflection of the joint.

The force plot in (d) clearly shows the fluctuation of displacement across the springs and dampers at 0 s.conds due to the inertial lag. The drop in stiffness at 0.25 seconds causes the quick change in the average force of the dampers. The effect of contact separates the damper forces, but the average value continues once the commanded motion has stopped. The spring forces change as would be suggested by the deflections in (c). The average force drops with the commanded stiffness and the two springs separate due contact with the constraint. Notice that spring 2 is deflected beyond the peak maximum negative force value and begins to rise. The equilibrium values correspond to the final set stiffness along with a small difference due to the small final equilibrium deflection of the joint. 
The resulting joint torque in (e) combines the effects of the phenomena suggested in each of the plots above. There is an initial joint torque at $\mathbf{0}$ seconds to overcome the inertia of the arm. When contact is made, the torque increases and oscillates slightly. This oscillation of the arm is not as evident on the other plots. The torque peaks at approximately 0.5 seconds. This corresponds with the point where the arm pushes the furthest into the constraint surface in (b). The torque then drops quickly due to the loss of damping force as the commanded position change ceases. This phenomenon illustrates the importance of plotting the spring torque. The quick drop in torque is clearly associated with damping only. From this point the torque drops with the stiffness and settles to a small constant value associated with the equilibrium deflection of the arm and the joint stiffness.

The total energy expenditure is approximately 6.3 J. Most of this energy is required by the branch 1 actuator. Notice that the energy expenditure begins with motion of the arm even before contact is made. This again points out the flaw in the configuration in which pure motion requires the expenditure of energy. The constant power drain for the motion is also seen in (h). Both branches greatly increase power requirement upon contact. The sudden drop in power at 0.5 seconds is associated with the loss of damping force when the commanded position change stops. The power for branch 2 increases at this point because the damper force is no longer working against the compressive spring force. The peak power for this task is $16.5 \mathrm{~W}$.

\section{Configuration 2}

The results for this configuration are shown in Figure 6.21. The change of inputs is more clearly associated with the commanded position and stiffness changes than with Configuration 1. The position change takes place from 0 to 0.5 seconds while the stiffness drops between 0.25 seconds and 0.75 seconds. Plots (b) through (f) are all identical te those of Figure 6.20 due to the similarity of the configurations. There are, 
however, slight differences in the en-rgy and power requirements in (g) and (h). There are no initial energy or power requirements for pure motion of the arm. Once contact is made, the total energy and power requirement change identically to those of Configuration I. The initial lack of required energy slightly reduces the total energy expenditure of the task to approximately 6.2 Joules. Also, almost all of the energy and power consumed in the task is required by the stiffness actuator. This is because a decrease in stiffness requires the stiffness actuator to push against the sum of the compressive forces in the springs. This is true of any decrease in stiffness in which the springs are operating in the compressive (negative) force range. Thus it is not unique to this task. There is also a relatively small energy expenditure and power requirement in the position actuator due to the defiection of the arm. This requirement ceases when the commanded notion stops at 0.5 seconds. Power requirements still peak at $16.5 \mathrm{~W}$, as in Configuration 1 , since it is associated with the change in stiffness.

\section{Configuration 3}

The results for this configuration are shown in Figure 6.22. Plots (a) through (f) are identical to those in Figure 6.21, again with the forces and displacements reciprocally scaled. The most noticeable results of this configuration are dramatic drop in energy and power requirements in $(\mathrm{g})$ and $(\mathrm{h})$. The large power and energy requirements for stiffness change, seen in the previous configurations, are no longer present. Energy expenditure is reduced to only approximately $0.16 \mathrm{~J}$ while power peaks at $0.9 \mathrm{~W}$. This task shows the ability of the third spring to eliminate the high forces associated with changing stiffness, as in Tasks I and 2. The position actuator consumes all of the energy and power requirements for this task. Note that the magnitude and shape of plots $(\mathrm{g})$ and $(\mathrm{h})$ are the same as those of the position actuator in Figure 6.21. In this case, the plot is enlarged due to the removal of the stiftiness actuator requirements. 


\section{Confipuration 4}

The results for this configuration are shown in Figure 6.23. Plots (a) through (f) are identical to Configuration 2 in Figure 6.21 and scaled from Configuration 3 . This scaling of force and displacement does not affect the external view of the system. In other words, the position, stiffness, joint torque, energy, and power all remain the same as in Configuration 3. 

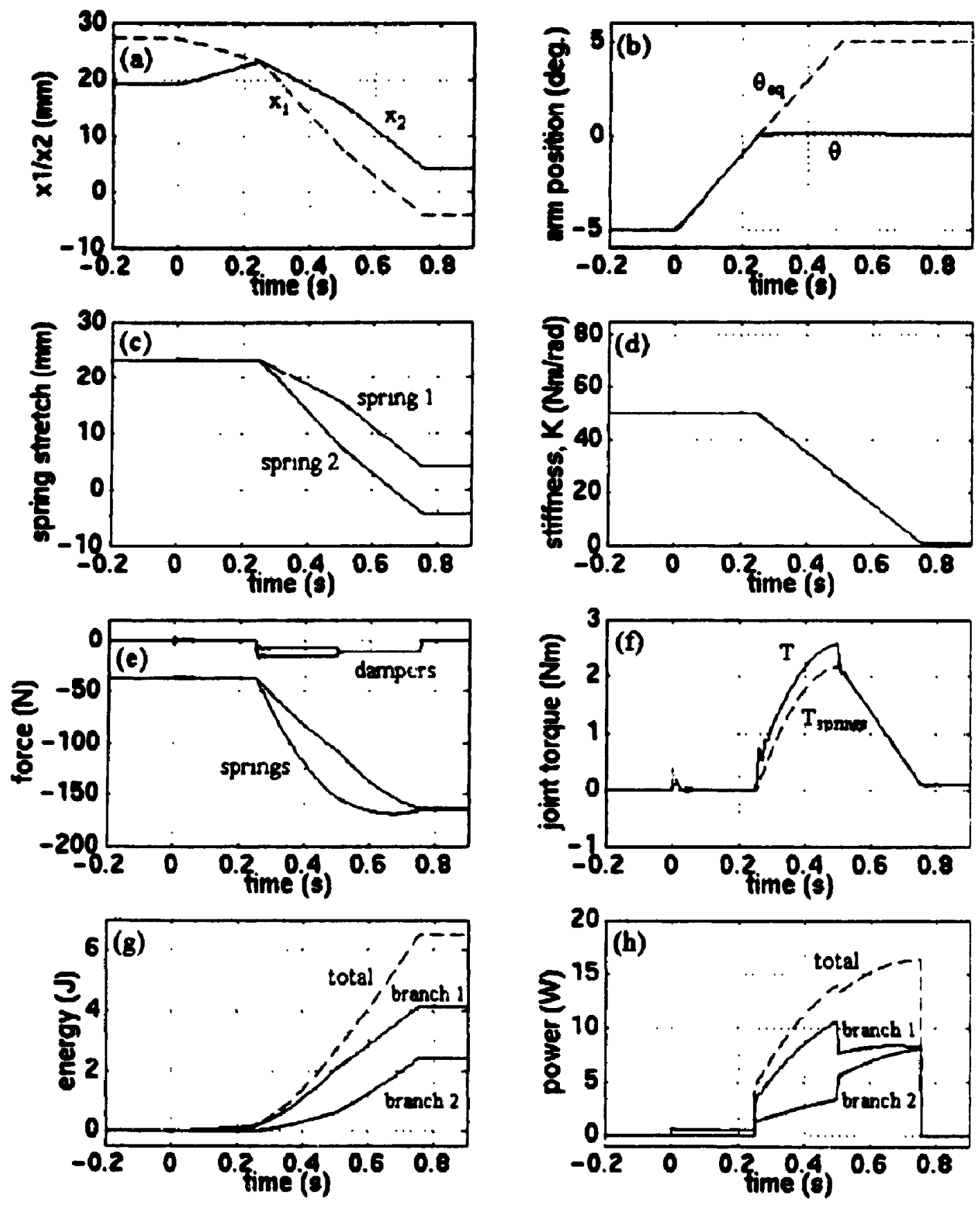

Figure 6.20 Task 4, Configuration 1 results, (a) $x_{1}$ and $x_{2}$, (b) $\theta_{e q}$ and $\theta$, (c) $\delta_{1}$ and $\delta_{2}$, (d) $K^{*}$, (e) spring and damper forces, (f) net joint torque and joint torque due to springs only,

(g) actuator output energy expenditure, (h) required actuator power 

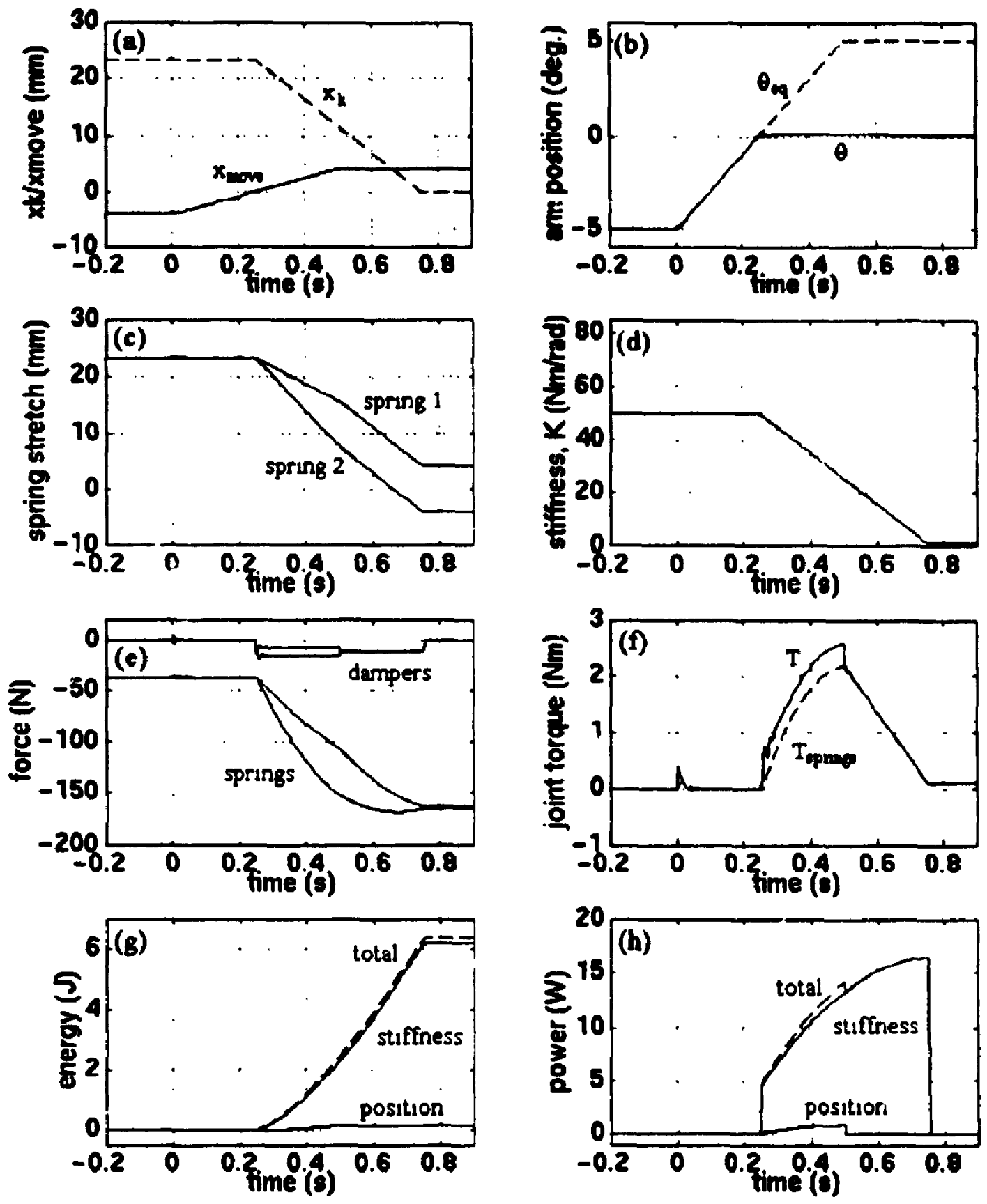

Figure 6.21: Task 4, Configuration 2 results, (a) $x_{k}$ and $x_{\text {move }}$ (b) $\theta_{\mathrm{eq}}$ and $\theta_{,}$(c) $\delta_{1}$ and $\delta_{2}$, (d) $\mathrm{K}^{*}$, (e) spring and damper forces, (f) net joint torque and joint torque due to springs only. (g) actuator output energy expenditure, (h) required actuator power. 

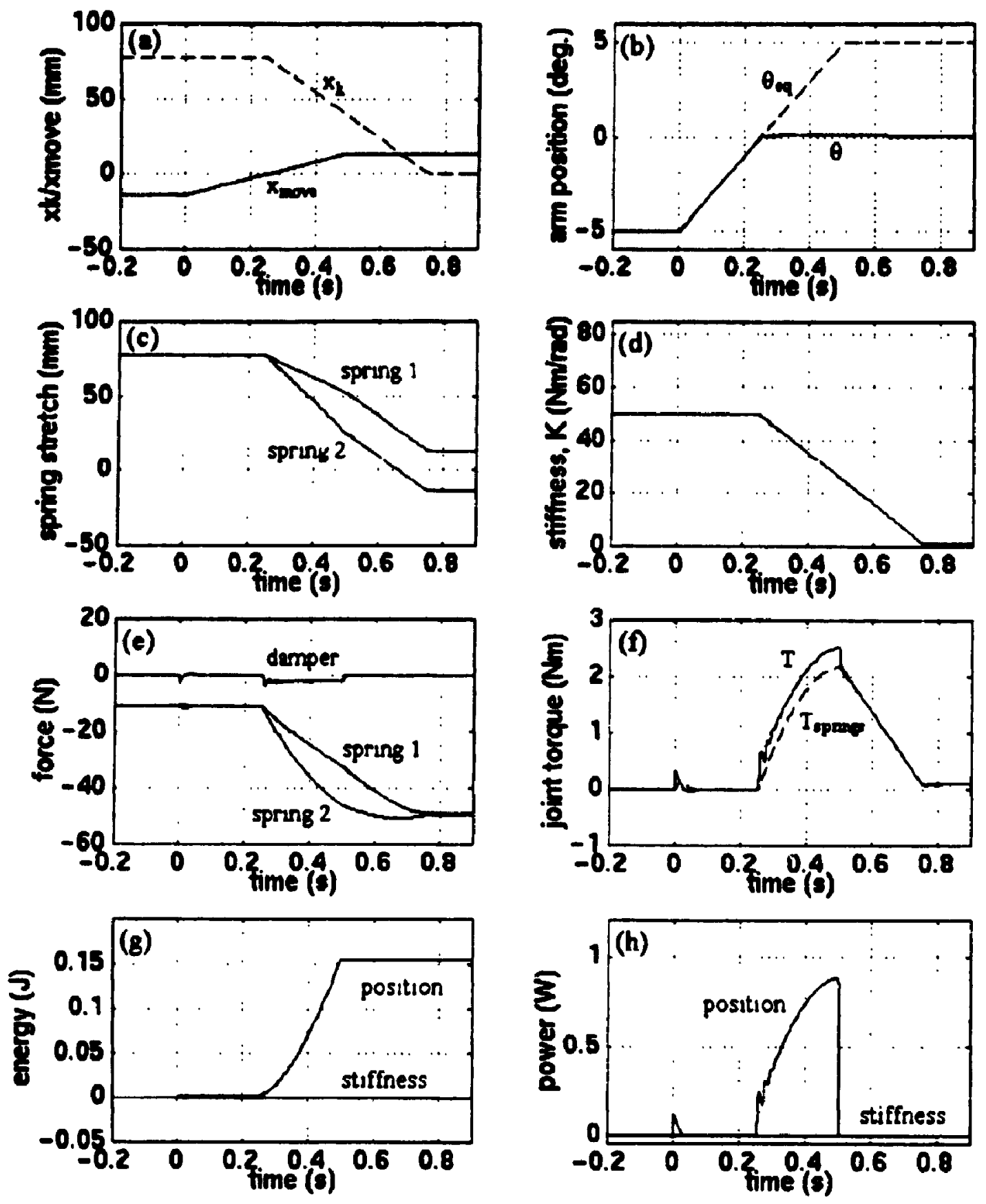

Figure 6.22: Task 4, Configuration 3 results, (a) $x_{t}$ and $x_{\text {move }}$ (b) $\theta_{\propto q}$ and $\theta$, (c) $\delta_{1}$ and $\delta_{2}$, (d) $K^{*}$, (c) spring and damper forces, (f) net joint torque and joint torque due to springs only,

(g) actuator output energy expenditure, (h) required actuator power 

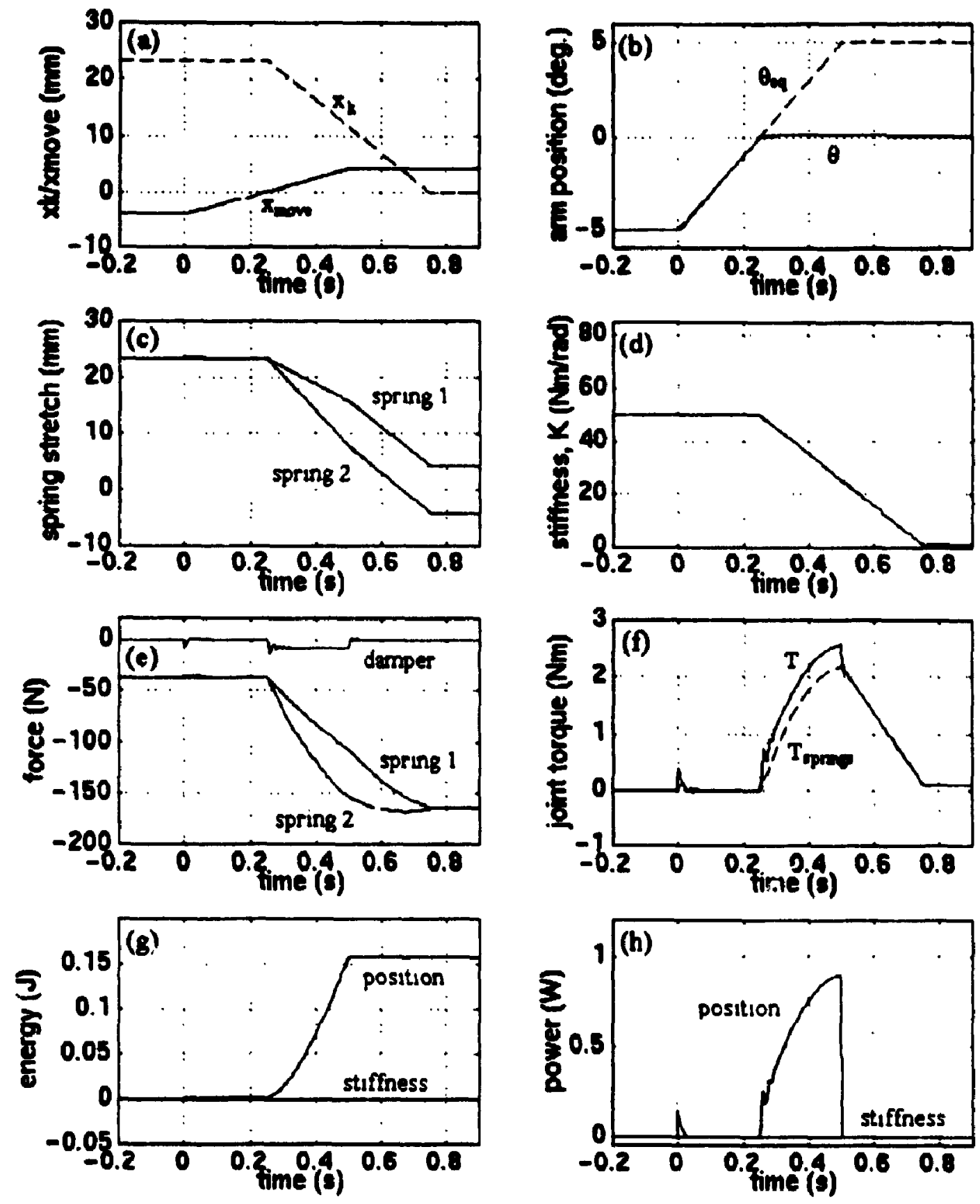

Figure 6.23: Task 4, Configuration 4 results, (a) $x_{k}$ and $x_{\text {move }}$ (b) $\theta_{\mathrm{cq}}$ and $\theta$, (c) $\delta_{1}$ and $\delta_{2}$, (d) $k^{*}$, (e) spring and damper forces, (f) net joint torque and joint torque due to springs only, (g) actuator output energy expenditure, (h) required actuatn- power 


\subsubsection{Summary of Simulation Results}

The simulations show a variety of performance characteristics. As seen in the results for each task, the extemal dynamic behavior is identical for each configuration. In general, the difference lies in the internal forces, energy expenditure, and power requirements. It is a common belief that biological organisms perform tasks using strategies that require minimurt energy. Such strategies are often sought for control of manipulators, especially in the field of prosthetics [Russell, i993, 1994]. Minimum energy is also vital to powered prostheses since energy has to be stored within the device, usually in batteries. Using minimum energy as a performance criterion, a comparison of the results is presented in Table $6 \mathrm{C}$.

Table 6C: Energy Expenditure of Simulated Tasks

\begin{tabular}{|c|c|c|c|c|}
\cline { 2 - 5 } \multicolumn{1}{c|}{} & \multicolumn{4}{c|}{ Total Energy Expenditure (Joules) } \\
\cline { 2 - 5 } \multicolumn{1}{c|}{} & Configuration 1 & Configuration 2 & Configuration 3 & Configuration 4 \\
\hline Task 1 & 2.3 & 2.3 & 1.1 & 1.1 \\
\hline Task 2 & 2.3 & 2.3 & 0 & 0 \\
\hline Task 3 & 0.31 & 0.0014 & 0.0014 & 0.0014 \\
\hline Task 4 & 6.3 & 6.2 & 0.16 & 0.16 \\
\hline
\end{tabular}

Configurations 1 and 2 show identical energy expenditures for changes in stiffness (Tasks 1 and 2). However, Configuration 2 is more efficient for pure motion (Task 3). Configurations 3 and 4 show identical energy expenditures for all tasks. Both are more efficient for changing stiffness (Tasks 1 and 2), and share the same reduced energy requirement for pure motion as Configuration 2 over that of Configuration 1 (Task 3). The combined effects of motion, stiffness change, and contact are shown in Task 4. Configurations 3 and 4 show an energy savings of almost 40 times over 
Configurations 1 and 2. The majority of this savings is associated with the change in stiffness.

Configurations 3 and 4 are therefore the most energy efficient. In reality, the two configurations are identical in form, scaled only by a lever ratio. Choosing the better configuration depends upon the relative importance of internal forces and the length of the springs.

178 

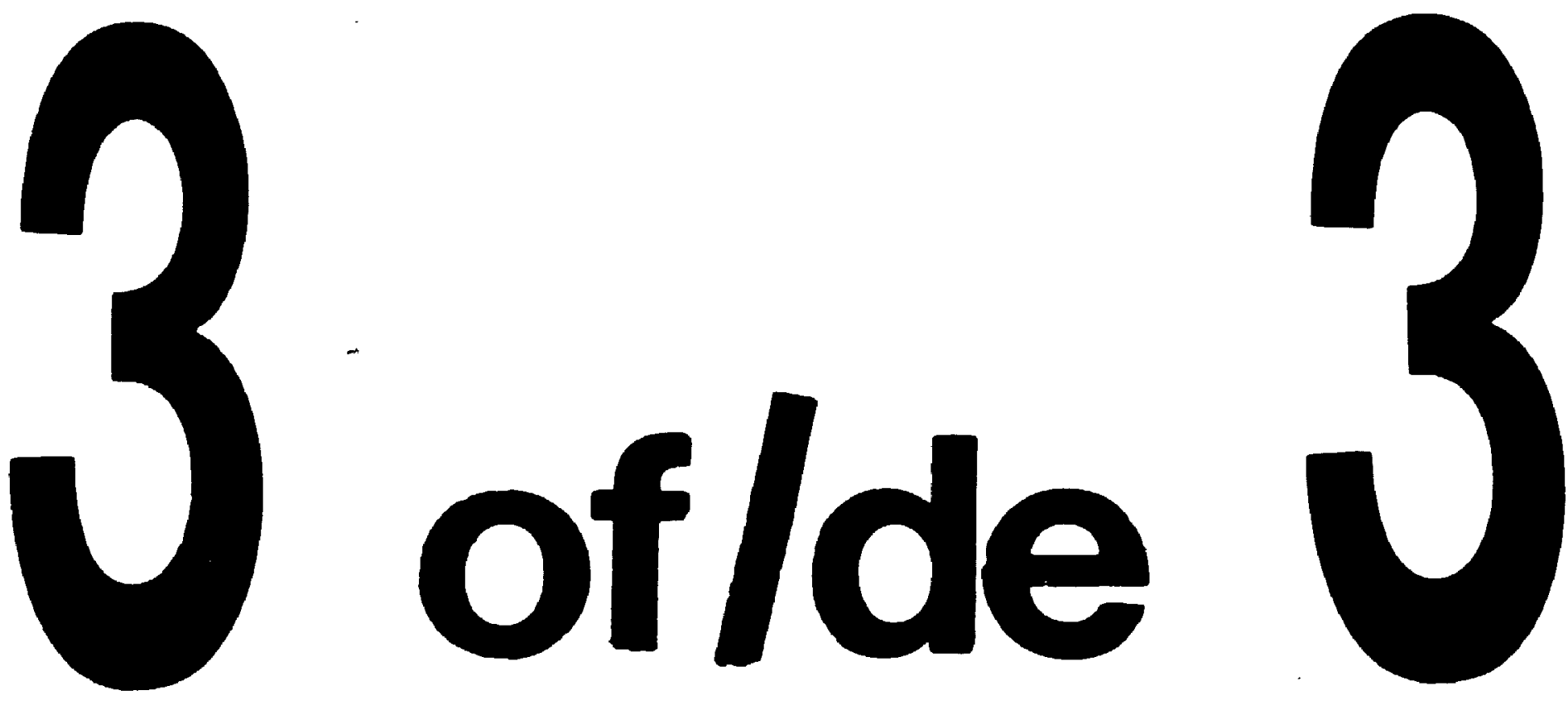

PM-1 3K"x4" PHOTOGRAPHK MICROCOPY TAREET NES 1010 ANSI/ISO \#2 EQUMALENT

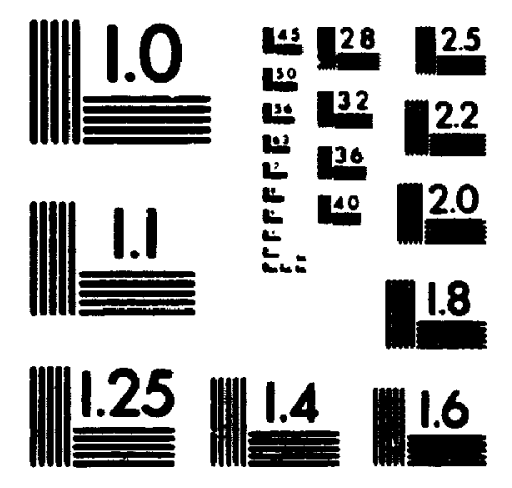

PAECIBIONEM RESOLUTION TARGETS 


\section{Chapter 7}

\section{Conclusions and Recommendations}

\subsection{Conclusions}

Antagonistic actuation permits the modulation of 'tiffness of a mechanical joint. Such an application has uses in both robotic and prosthetic technologies. In prosthetic research it is common to use the sum of antagonistic muscle activity to indicate the joint stiffness and the difference to indicate the equilibrium position. Ideally it is desirable to continue this sum and difference technique in control of the prosthesis stiffness. However, analysis of the joint system becomes more difficult with this restriction. Separation of the inputs into stiffness and position contributions, as in Section 3.1.2, allows direct analysis of the system in terms of separate effects. Responses of the system to stiffness or position changes can be viewed directly. This separation of contributions also demonstrates that quadratic springs (force proportional to the square of deflection) are necessary in order to decouple joint stiffness and deflection. Section 4.1 develops the proof of this requirement. Furthermore, equation (4-33) reveals that the sum and 
difference technique can only be used if the antagonistic springs are identical. The resulting joint acts as a linear torsion spring with a variable stiffness (see Figure 3.7(b)).

One of the problems associated with the implementation of variable stiffness using mechanical components is the physical limitations associated with the geometry. For most applications, joints are not often required to be able to rotate indefinitely. In a prosthetic application, for example, the human arm is only able to rotate approximately 140 degrees. It is suggested that the equilibrium position of the arm is able to rotate a certain distance beyond this limit in order to produce torques at both extremes. However, this too has a finite limit. These extremes must be considered when implementing the antagonistic actuation system. The components of the system must be able to accommodate motion to all required extremes. In addition, the stiffness elements also have physical limitations in the form of maximum positive and negative deflections. Section 4.2 examires the effect that these physical limitations have on the performance of the arm.

Design criteria can define a minimum and maximum stiffness required for the joint. The torque and deflection would ideally be unlimited at any given stiffness between these extremes. Additional criteria may place an upper limit on required torque or deflection for a given stiffness. For example, the maximum joint deflection that is physically possible is the case where equilibrium position is at one extreme and the joint is forced to the opposite position extreme. Such a case is expected for low joint stiffness. However, at high stiffness it is not expected that such large deflections are possible. In fact, study of human interaction suggests that most contact torques are created at low stiffness. In general, high stiffiness is used for unconstrained motion. Thus only minimal torques and deflections are required at high stiffness. This is fortunate for the application of the design suggested here. Analysis of the geometry shows that both stiffiness elements are stretched to their upper limit at maximum joint stiffness. This means that no deflection is possible and the joint is effectively rigid. At any given stiffiness there is a 
maximum deflection that can be applied before one of the stiffiness elements reaches an extreme limit. The torque that can push the joint to this limit is also associated with the given stiffiness. The joint can only work as a variable stiffiness actuator within these limitations. It is effectively rigid beyond the maximum deflection. This limitation results in a stiffness controlled region of the torque-deflection curve for the joint. Figure 4.11 shows the effect of this region on the performance of the joint.

Section 4.4 reveals a correlation between the shape of the stiffiness controlled region and the shape of the force function of the stiffiness elements, assuming that the two branches are identical. In general, the shape of the force function is inverted to get the upper limit of the stiffness controlled region. The limits also depend on the ratio between maximum stretch and compression of the springs. A small maximum compression has the effect of cutting the end off the stiffness controlled region as seen in Figure 4.15(a). As well, the deflection limit depends on other design parameters. The general shape of the stiffness controlled region is useful, however, in determining the ability of stiffness elements to provide sufficient performance. For instance, springs whose force function approaches linear provide poor stiffiness controlled regions. Force functions that are more curved provide larger stiffiness controlled regions, but too sharp of a curve requires high precision to set the joint stiffiness.

Study of human interaction suggests that large contact forces are dealt with at low joint stiffness for the sake of stability. This implies that if there is a maximum torque that the joint can apply, it should occur at low stiffness. Section 4.4 shows that this maximum torque is equivalent to the product of the joint radius and the range of force in the stiffness element. Such a criterion results in a desired stiffiness control region as shown in Figure 4.17.

Section 4.3.1 deals with the degree to which joint stiffiness and endpoint deflection are related. A small variance from the linear stiffness function, i.e., quadratic force function, can create relatively large errors in the joint stiffiness. However, these 
errors may be considered acceptable design compromises. For instance, Figure 4.12 includes the spring stiffiness functions that cause a $100 \%$ increase or decrease in joint stiffiness when the arm is deflected by 90 degrees. At low stifiness this change is not very significant. An increase from $1 \mathrm{~N} \cdot \mathrm{m} / \mathrm{rad}$ to $2 \mathrm{~N} \cdot \mathrm{m} / \mathrm{rad}$ would not dramatically affect the performance of the arm. At high stiffiness, this same change does become significant. However, deflections are not as large at high stiffiness, in general, and therefore the errors are smaller. The same torque that causes a 90 degree deflection at low stiffiness may only cause a small deflection, and error, at higher stiffness. Thus the error may again be insignificant. Also, with position feedback the set stiffness can be lowered to offset the effect of endpoint deflection.

Study of the power and energy requirements suggests modifications of the design. First, the branches should be connected around a second radius such that the forces in the branch springs are used to work against one another to eliminate a net torque on the actuator, as in Figure 4.7. This eliminates any energy required for pure motion. The work done on one branch is equivalent to the work done by the other branch. This significantly reduces the energy requirements for motion, as shown by simulations of motion in Figure 6.12(g) and 6.13(g). A second feature of this configuration is the direct separation of motion and stiffness inputs. Each input is controlled by an actuator. This allows the size of each actuator to be based on the separate requirements of motion and stiffiness. In other words, the stiffness actuator can be sized based on the requirements of stiffiness changes while the position actuator can be sized based on motion requirements.

An additional property suggested by power and energy requirements is that the force function of the individual springs remain as close to zero as possible for most of the spring stretch. This causes the smallest power requirements and thus smaller actuators. Designing the force function in this manner has a tradeoff between decoupling of the stiffiness and deflection. The relationship is developed using the power savings index of equation (4-140). Using the parameters selected in Chapter 6, the power savings index is 
approximately a maximum of $10 \%$ for a stiffiness error of $100 \%$ at 90 degrees deflection. Once again, this error may be acceptable to the design. Note, however, that the technique used to derive this power savings index does not take into accoum a constant range of force available from the springs (sce Figure 4.20). Taking this into account, the power savings may be negligible.

Section 4.5.4 suggests an additional method of reducing power and energy requirements. The addition of a third spring to offset the sum of forces created in the branch springs significantly reduces, or eliminates, the net force placed on the stiffness actuator. The only force that remains is that due to loading of the joint. This is similar to the elimination of pure motion energy requirements b; connecting the branches. However in this case, the energy is stored in a third spring rather than instantly transferred. The work done on the branch springs to stretch them is equivalent to the work done by the third spring. Conversely, the work done by the branch springs as they become less stretched is equal to the work done on the third spring. The drawback to this modification is the extra space required for the third spring. In fact, if the branch springs are developed to produce the maximum force that spring design will allow for that size, the third spring will either have to be twice as big, or use two springs to offset the total force of the branch springs. This may double the space required for the entire system.

Perhaps the largest difficulty in the design is the creation of the quadratic springs. The force function suggested by equation (4-50) not only varies quadratically, but also includes compressive (negative) forces for a portion of the positive displacement and a negative stiffness near the maximum negative displacement. Approximate quadratic force-deflection relationships are difficult, but not impossible, to create using conventional spring design. The compressive force for positive deflection intuitively seems impossible by these methods, since the physical meaning corresponds to pulling on the spring and having the spring force push with you. However, the "zero displacement" position on the spring is arbitrary. It could be defined at some point of the compressive 
region of displacement rather than at the "zero force" position. Thus the spring is initially loaded. The most difficult property to design is the negative stiffness. This is impossible by conventional methods.

A possible solution to all the spring design problems is the use of rolamites. It is possible to design any desired force-deflection relationship into these devices. This includes quadratic shapes, compressive forces for positive displacements, and negative stiffiness. In addition, the design is relatively simple when compared to conventional methods. The force-deflection relationship is created by cutting an appropriate shape, similar to the shape of the function itself, out of a band of flexible material. The band is then attached around rollers as in Figure 5.1.

While a rolamite device can be made to fit the desired function, it is limited to a maximum ferce that a given size and band material can create. Section 6.1 indicates that the use of roiamites for a prosthetic application of the joint provides unsatisfactory results. The limiting torque is a maximum of $3.2 \mathrm{~N} \cdot \mathrm{m}$ using the space allotted for the springs. This result is based on the extreme values of parameter selection. It does not take into account the space that would be required for other components of the design, such as actuators, gearing, batteries, or a controller. This suggests that rolamites, in the present form, are not sufficient for a prosthetic implementation of the basic design. However, it does not exclude the possibility of using them in a robotic application. Such applications are not as limited for space. Acceptable results may also be achieved by adapting the rolamite design. For instance, size restrictions may be improved by redesigning the rolamite to operate in a circular motion.

The selection of parameters in Chapter 6 also suggests another improvement to e basic system configuration. Much space can be saved by moving the position actuator to the opposite end of the branches so that it is between the components and the joint, as seen in Figure 6.3. The previous configurations require a large amount of space for the translation of the branch springs as the equilibrium position changes. In the 
improved configuration, this space can be either eliminated or can be used to increase the size, and thus force, of the springs. In fact, this adaptation is necessary to produce any reasonable maximum torque using the rolamites. Without this adaptation, the maximum limiting torque is in the magnitude of hundredths of a $N \cdot m$.

The performance of the system is simulated in Section 6.2. The simulations show the performance of each of the configurations suggested throughout this thesis. The behavior of the system from the point of view of the environment does not depend on the configuration chosen. For eacn task, the arm position, stiffness, and joint torque are always identical among the configurations. The difference between configurations is apparent in the internal forces, energy expenditure, and power requirements.

Configuration 1 (Figure 6.5) consumes the most energy. This is expected because it requires energy to perform pure motion with no loading. The connection of branches in Configuration 2 (Figure 6.6) eliminates this need. Configurations 3 and 4 (Figure 6.7) eliminate most of the energy required to change stiffness. The tradeoff between them is the size versus internal forces. Configuration 3 uses longer springs that occupy the same space as the entire systcm of Configurations 1 and 2. These longer springs allow the use of less force by increasing the radius of the joint. (Note that the joint radius is automatically increased by the changing parameters via equation (4-77).) Configuration 4 uses the same spring size, and thus force, as Configurations 1 and 2. However, since the space for translation of the springs in the first two configurations is removed, the system in Configuration 4 occupies much less space (approximately one third). Certainly Configurations 3 and 4 show much improvement over the first two, but they require more complicated design of the joint region. The selection of the appropriate configuration depends mainly on the ability of available springs. The springs used in each simulation create forces that are much larger than can be produced by the rolamites suggested above. Implementation of the system requires the creation of springs that are able to produce sufficient force. 


\subsection{Recommendations}

The main obstacle in implementation of the design is the creation of the quadratic springs. Study of the rolamite geometry may suggest a possible solution. For instance, it may be possible to adapt the roller-band concept to a circular configuration where the springs are allowed to rotate a number of times for one stretch of the spring. This is equivalent to a long linear rolamite. The increase in length allows a larger joint radius (by equation (4-77)) and reduced spring forces. In addition, it may be possible to create quadratic springs by another method. For instance, a quadratically varying radius stretching a linear spring will have the same effect as a quadratic spring.

A study of available actuators may select an appropriate method. The typical actuators currently used in robotics and prosthetics are dc-motors. There are a variety of alternative actuation methods, most of which are experimental (see Section 2.2.1, for example). One of these techniques may prove to be ideal for the use in this design.

Although simulations of the system show no problems, it is necessary to perform a proper stability analysis of the system. The individual spring function design includes negative stiffness which, by definition, is unstable. However, both springs do not operate in that region at the same time. This requires the setting of joint stiffness to be negative. It appears from the simulations that the operation of one spring in the negative stiffness region does not cause instability so long as the combined stiffness at the joint is positive. The results of the simulations are the only basis for this assumption. A proper study may guarantee this property or set some other requirements for the stability of the system.

The implementation of this system into a prosthesis or robot manipulator requires a controller. The concept of the design applies techniques suggested by impedance control. However, the typical implementation of impedance control calculates impedance properties within the controller and applies the appropriate torque through an actuator. Since the impedance is mechanically variable in this design, the control 
scheme must be modified to include this operation. In addition, the application of the design to a prosthesis requires the collection and processing of muscle activity to determine desired stiffness and equilibrium position.

In robotics, a manipulator usually has more than one joint. Allowing variable stiffness at each joint creates a stiffness ellipse at the endpoint. This means that the stiffness of the arm from the point of view of the environment depends on the orientation of the endpoint and the direction of the contact forces. Decisions must therefore be made on what stiffness to set each joint. Study of a multijoint variable stiffness system is therefore the next step in implementation of the mechanically variable joint stiffness technique proposed in this thesis for use in robotic systems.

187 


\section{References}

Abul-Haj, C.J., Elbow-Prosthesis Emulation: A Technique for the Quantitative Assessment of an Assistive Device, Ph.D. thesis, Department of Mechanical Engineering, M.I.T., Cambridge, MA, June 1987.

Abul-Haj, C.J., and N. Hogan, Functional Assessment of Control Systems for Cybernetic Elbow Prostheses - Part I: Description of the Technique, IEEE Transactions on Biomechanical Engineering, vol. 37, no. 11, pp. 1025-1036, 1990a.

Abul-Haj, C.J., and N. Hogan, Functional Assessment of Control Systems for Cybernetic Elbow Prostheses - Part II: Application of the Technique, IEEE Transactions on Biomechanical Engineering, vol. 37, no. 11, pp. 1025-1036, 1990b.

An, C.H. and J.M. Hollerbach, Dynamic Stability Issues in Force Control of Manipulators, Proceedings of the IEEE International Conference on Robotics and Automation, pp. 890-96, 1987.

Andrews, J.R. and N. Hogan, Impedance Control as a Framework for Implementing Obstacle 1voidance in a Manipulator, Control of Manufacturing Processes and Robotic Systems, NY, ASME, pp. 243-51, 1983.

Bell, L.E., Rolamire Design Using Figures of Merit, Proceedings of the First Symposium on Rolamite, Albuquerque, NM, pp. 79-84, Nov. 12-13, 1968.

Cadman, R.V., Rolamite - Geometry and Force Analysis, Transactions of the ASME, Journal of Engineering for Industry, pp. 186-192, February 1969.

Cadman, R.V., Compuation of Force Traces for the Rolamile, Transactions of the ASME, Journal of Engineering for Industry, pp. 47-52, February 1971.

Childress, D.S., Control Philosophies for Limb Prostheses, Progress in Bioengineering, J.P. Paul, J.C. Barbenel, J.M. Courtney, and R.M. Kenedi, editors, Adam Hilger, N.Y., pp. 210-215, 1989.

Chironis, N.P., Fiendishly Simple Roller-band Device Challenges Established Mechanisms, Product Engineering, Vol. 38, No. 23, pp. 80-83, November 6, 1967.

Reprinted in Mechanisms and Mechanical Devices Sourcebook, McGraw Hill, pp. 164170, 1991.

Colgate, E., The Control of Dymamically Interacting Systems, Ph.D. dissertation, Department of Mechanical Engineering, M.I.T., August 1988. 
Colgate, E., On the Inherent Limitations of Force Feedback Compliance Controllers, Robotics Research, Youcef-Tourni, K., and Kazerooni, H., editors., ASME, Dec. 1989.

Colgate, E., and N. Hogan., An Analysis of Contact Instability in Terms of Passive Physical Equivalents, Proceedings of the IEEE International Conference on Robotics and Automation, IEEE Press, 1989.

Cutkosky, M.R., and P.K. Wright, Active Control cf a Compliant Wrist in Manufacturing Tasks, Journal of Engineering for Industry, Transactions of the ASME, no. 108, pp. 3643, February 1986.

Dolan, J.M., M.B. Friedman, and M.L. Nagurka, Dynamic and Loaded Impedance Components in the Maintenance of Human Arm Posture, IEEE Transactions on Systems, Man, and Cybernetics, vol. 25, no. 3, pp. 698-709, May-June 1993.

Drake, S.K., and S.N. Sumunovic, The U/se of Compliance in a Robot Assembly System, IFAC Symposium Information and Control Problems in Manufacturing Technology, Tokyo, 1977.

Eppinger, S.D., and W.P. Seering, On Dymamic Models of Robot Force Control, Proceedings of the IEEE International Conference on Robotics and Automation, pp. 2934, 1986.

Eppinger, S.D., and W.P. Seering, Understanding Bandwidth Limitations in Robot Force Control, Proceedings of the IEEE International Conference on Robotics and Automation, pp. 904-09, 1987.

Eppinger, S.D., and W.P. Seering, Three Dynamic Problems in Robot Force Control, Proceedings of the IEEE International Conference on Robotics and Automation, pp. 392 97, 1989.

Eulert, D.D., editor, Proceedings of the First Symposium on Rolamite, Albuquerque, NM, Nov. 12-13, 1968.

Feldman, A.G., Functional Tuning of the Nervous System During Control of Movement or Maintenance of Steady Posture: Controllable Parameters of she Muscles, Biophysics, no. 11, pp. 667-675, 1966.

Feldman, A.G., Once More on the Equilibrium-point Hypothesis (ג-Model) for Motor Control, Journal of Motor Behavior, no. 18, pp. 17-54, 1986.

Field, G., and Y. Stepanenko, Model Reference Impedance Control of Robolic Manipulators, IEEE Pacific Rim Conference, pp. 614-17, 1993. 
Flash, T., and N. Hogan, The Coordination of Arm Movements: An Experimentally Confirmed Mathematical Model, The Journal of Neuroscience, no. 5, pp. 1688-1703, 1985.

Gorinevsky, D.M., Modeling of Direct Motor Program Learning in Fast Human Arm Motions, Biological Cybernetics, vol. 69, pp. 219-228, 1993.

Goswami, A., M.A. Peshkin, and J.E. Colgate, Passive Robotics: An Exploration of Mechanical Computation, Proceedings of the 1990 IEEE International Conference on Robotics anc Automation., IEEE Press, 1990.

Greenhill, S., The Digit Muscle, Industrial Robot, vol. 20, no. 5, pp. 29-30, 1993.

Hanna, J.R., and J.H. Rowland, Fourier Series Transforms and Boundary Value Problems, 2nd ed., John Wiley and Sons, Toron'o, 1990.

Hill, A.V., The Heat of Shortening and the Dymamic Constants of Muscle, Proc. R. Soc., 126B, pp. 136-195, 1938.

Hirzinger, G., Direct Digital Robol Control Using a Force-Torque Sensor, Proceedings of the IFAC Symposium on Real Time Digital Control Applications, pp. 243-255, 1983.

Hogan, N., Mechanical Impedance Conirol in Assistive Devices and Manipulators, Proceedings of the 1980 Joint Automated Control Conference, San Francisco, TA 10-B, 1980a.

Hogan, N., Tuning Muscle Stiffness Can Simplify Control of Natural Movement, 1980 Advances in Bioengineering, Van C. Mow, editor, ASME, New York, 1980b.

Hogan, N., Prostheses Should Have Adaptively Controllable Impedance, IFAC Symposium on Control Aspects of Prosth. and Orthot., Columbus, OH, pp. 155-162, May 1982.

Hogan, N., Adaprive Control of Mechanical Impedance by Co-activation of Antagonist Muscles, IEEE Transactions on Automatic Control, AC-29(8), pp. 681-90, 1984a.

Hogan, N., Impedance Control of Industrial Robots, Robotics Computer Integrated Manufacturing, vol. 1, pp. 97-113, $1984 \mathrm{~b}$.

Hogan, N., Impedance Control: An Approach to Manipulation, Part I - Theory, Journal of Dynamic Systems, Measurement and Control, vol. 107, pp. 1-7, 1985a.

Hogan, N., Impedance Control: An Approach 10 Manipulation, Part II - Implementation, Journal of Dymamic Systems, Measurement and Control, vol. 107, pp. 8-16, 1985 b. 
Hogan, N., Impedance Control: An Approach to Manipulation, Part III - Applications, Joumal of Dynamic Systems, Measurement and Control, vol. 107, pp. 17-24, 1985c.

Hogan, N., Stable Execution of Contact Tasks Using Impedance Control, Proceedings of the IEEE Conference on Robotics and Automation, pp. 1047-54, 1987.

Hogan, N., On the Stability of Manipulators Performing Contact Tasks, IEEE Journal of Robotics and Automation, vol. 4, no. 6, pp. 677-86, Dec. 1988.

Houk, J.C., Regulation of Stiffness by Skeletomotor Reflexes, Ann. Rev. Physiol., no. 41, pp. 99-114, 1979.

Hudgins, B.S, P.A. Parker, and R.N. Scott, A New Strategy for Mulifunction Myoelectric Control, IEEE Transactions in Biomedical Engineering, vol. 40, no. 1, Jan. 1993.

Hunter, I.W., and S. Lafontaine, A Comparison of Muscle with Artificial Actuators, Transactions of the IEEE, pp. 178-185, 1992.

Immega, G.B., Romac Actuators for Micro Robots, 1987 IEEE Micro Robots and Teleoperators Workshop, IEEE Press, 1987.

Jacobsen, S.C., and R.W. Mann., Control Systems for Artificial Arms, Proceedings of the 1973 International Conference on Cybernetics and Society, Boston, MA, pp. 298-303, Nov. 1973.

Jacobsen, S.C., Knutti, D.F., Johnson, R.T., and Sears, H.H., Development of the thah Artificial Arm, IEEE Transactions on Biomedical Engineering, BME-29, no. 4, pp. 24969, April 1982.

Jacobsen, S.C., J.E. Woods, D.F. Knutti and K.B. Biggers, The (hah M.I.T. Dextrous Hand: Work in Progress, The International Journal of Robotics Research, vol. 3, no. 4, Winter 1984.

Jacobsen, S.C., E.K. Iversen, D.F.Knuttti, R.T. Johnson, and K.B. Biggers, Design of the Utah M.I.T. Dextrous Hand, IEEE International Conference on Robotics and Automation, pp. 1520-1532, 1986.

Jacobsen, S.C., H. Ko, E.K. Iversen and C.C. Davis, Antagonistic Control of a Tendon Driven Manipulator Arm, Proceedings of the IEEE International Conference on Robotics and Automation, pp. 1334-39, 1989.

Kaneko, M., N. Imamura, K. Yokoi and K. Tanie, Direct Compliance Control of Manipulator Arms - Basic Concept and Application Examples, IFAC Robot Control, pp. 365-70, 1988. 
Kamopp, D.C., D.L. Margolis, and R.C. Rosenberg, System Dynamics: A Unified Approach, 2nd ed., John Wiley \& Sons, 1990.

Katic, D. and M. Vukobratovic, Learning Impedance Control of Manipulation Robots by Feedforward Comnectionist Siructures, IEEE, pp. 45-50, 1994.

Kazeroonı, H., T.B. Sheridan, and P.K. Houpt, Robust Compliant Motion for Manupulators. Part I: The Fundamental Concepts of Compliant Motion, IEEE Joumal or Robotics and Automation, vol. RA-2, no. 2, pp. 83-92, 1986a.

Kazerooni, H., P.K. Houpt and T.B. Sheridan, Robust Compliant Motion for Manipulators, Part II: Design Method, IEEE Journal of Robotics and Automation, vol. RA-2, no. 2, pp. 93-105, 1986b.

Kazerooni, H., Robust, Non-Linear Impedance Control for Robot Manipulators, Proceedings of the IEEE International Conference on Robotics and Automation, pp. 74150, IEEE Press, 1987.

Khatib, O., and J. Burdick, Motion and Force Control of Robot Manipulators, Poceedings of the IEEE International Conference on Robotics and Automation, pp. $1,81-1386,1986$.

Khatib, O., A Unified Approach for Motion and Force Control of Robot Manipulators: The Operational Space Formulation, IEEE Journal of Robotics and Automation, RA3(1): pp. 43-53, February 1987.

Komechi, S., and V. Zschorlich, The Nature of the Stabilizing Functions of Skeletal Muscles, Journal of Biomechanics, vol. 27, no. 2, pp. 215-225, 1994.

Lan, Ning, and Patrick E. Crago, Optimal Control of Antagonistic Muscle Stiffness During Voluntary Movements, Biological Cybernetics, vol. 71, pp. 123-135, 1994.

Latash, M.L., and V.M. Zatsiorsky, Joint Stiffness: Myth or Reality?, Human Movement Science, vol. 12, pp. 653-692, 1993.

Latre, L.G., and M.A. Cabrera, Influence of Joint Flexibility in the Adjustment of Impedance Coniroller Parameters, Proceedings of the 30th Conference on Decision and Control, Brighton, England, pp. 2775-80, December 1991.

Latwesen, A., and P.E. Patterson, Identification of the Lower arm Motions Using the EMG Signals of Shoulder Muscles, Med. Eng. Phys., vol. 16, pp. 113-121, March 1994.

Laurin-Kovitz, K.F., J.E. Colgate, and S.D.R. Cames, Design of Components for Programmable Passive Impedance, Proceedings of the 1991 IEEE International Conference on Robotics and Automation, Sacramento, CA, pp. 1476-81, April 1991. 
Lawrence, D.A., Actuator Limitations on Achievable Manipulator Impedance, Transactions of ti:e IEEE, pp. 560-565, 1989.

LeBlanc, M.A., Making the Case for Body-Powe-ed Upper-Limb Prostheses, Proceedings of the 10th Annual Conference on Rehabilitation Technology, RESNA, pp. 196-198, June 1987.

Lee, Y.T., H.R. Choi, W.K. Chung and Y. Youm, Stiffness Control of a Coupled TendonDriven Robot Hand, IEEE Control Systems, pp. 10-19, 1994.

Lu, Z., and A.A. Goldenberg, Implementation of Robust Impedance and Force (ontrol, Journal of Intelligent and Robotic Systems, pp. 145-163, 1992.

Ma, S., S. Hirose, and H. Yoshinada, Design and Experiments for a Coupled TendonDriven Manipulator", IEEE Control Systems, pp. 30-36, February 1993.

Mann, R.W., Cybernetic Limb Prostheses: The ALZA Distinguished Lecture, Annals of Biomedical Engineering, vol. 9, pp. 143, 1981.

Mansfield, J.M., The Design of a Lightwetght Elbow Prosthesis Emulator, Master's Thesis, Dept. of Mechanical Engineering, M.I.T., Cambridge, MA, May 1988.

Mason, M.T., Compliance and Force Control for Computer Controlled Manipulators, IEEE Transactions on Systems, Man, and Cybernetics, SMC-11(6), pp. 418-32, June 1981.

Meghdari, A., M. Jafarian, M. Mojarred, and M. Shahinpoor, Exploring Arlıficial Muscles as Actuators for Artıficial Hands, Intelligent Structures, Materials, and Vibrations, vol. 58, pp. 21-26, 1993.

Miller, C.M., A Method for Assessing the Importance of Elbow Dynamic Behavior in Manual Tasks, Master's thesis, Department of Mechanical Engineering, M.I.T., Cainbridge, MA, February 1987.

Mills, J.K., Hybrid Actuator for Robot Manipulators: Design. Control and Performunce, IEEE International Conference on Robotics and Automation, Cincinnati, pp. 1872-78, 1990.

Mittal, S., U. Tasch, and Y. Wang, A Redundant Actuation Scheme for Independent Modulations of Stiffness and Position of a Robotic Joint: Design. Implementation, and Experimental Evaluation", Advances in Robotics, Mechatronics, and Haptic Interfaces, vol. 49, pp. 247-256, 1993. 
Mussa-lvaldi, F.A., N. Hogan, and E. Bizzi, Neural, Mechanical, and Geometric Factors Subserving Arm Posture in Humans, The Journal of Neuroscience, vol. 5, no. 10, pp. 2732-2743, Oct. 1985.

Nevins, J.L. and D.E. Whitney, The Force Veclor Assembler Concept, Proceedings of the Ist CISM-IFTOMM Symposium on Theory and Practice of Robots and Manipulators, Udine, Italy, Sept. 1973.

Parker, P.A., and R.N. Scott, Myoelectric Control of Prostheses, CRC Critical Reviews in Biomedical Engineering, vol. 13, issue 4, pp. 283-310, 1986.

Paul, R.P., Problems and Research Issues Associated with the Hybrid Control of Force and Displacement, Proceedings of the IEEE International Conference on Robotics and Automation, pp. 1966-71, 1987.

Polit, A., and E. Bizzi, Characteristics of Motor Programs Underlying Arm Movements in Monkeys, Journal of Neurophysiology, no. 42, pp. 183-194, 1979.

Popat, R.A., D.E. Krebs, J.M. Mansfield, D.L. Russell, E.A. Clancy, K.M. Gill-Body, and N. Hogan, Quantuative Assessment of Four Men Using Above-Elbow Prosthetic Control, Archives of Phys. Med. and Rehab., no. 74, pp. 720-728, 1993.

Peshkin, M.A., Programmed Complaance for Error Corrective Assembly, IEEE Transactions on Robotics and Automation, vol. 6, August 1990.

Railbert, M.H. and J.J. Craig, Hybrid Position Force Control of Manipulators, Transactions of the ASME, Journal of Dynamics, Systems, Measurement and Control, vol. 102, pp. 126-33, June 1981.

Roberts, R.K., R.P. Paul, and B.M. Hillberry, The Effect of Wrist Force Sensor Stiffiness on the Control of Robot Manipulators, Proceedings of the IEEE International Conference on Robotics and Automation, pp. 269-274, 1985.

Rothchild, R.D., and R.W. Mann, An EMG Controlled, Force Sensing, Proportional Rate, Elbow Prosthesis, Proceedings of the Symposium of Biomedical Engineering, Marquette University, Milwaukee, Wisconsin, Vol. 1, pp. 106-109, June 1966.

Russell, D.L., Energv Expenditures in Upper Limb Prostheses Performing Constrained Movements, Proceedings of the Myo-Electric Control Symposium, pp. 98-104, 1993.

Russell, D.L., Myoelectric Control Using Modulated Minimum Jerk Moverment Segments, Proceedings of the UNB Myo-Electric Control Symposium, pp. 129-136, 1994. 
Russell, D.L., and C. English, Design Considerations for Variable Sriffness Prostheses, Proceedings of the UNB Myo-Electric Control Symposium, 1995.

Salisbury, J.K., Active Stiffness Control of a Manipulator in Cartesian Covrdinutes, Proceedings of the 19th IEEE Conference on Decision and Control, pp. 95-100, 1980.

Salisbury, J.K., and J.J. Craig, Articulated Hands: Force Control and Kinematic Issues, International Journal of Robotics Research, vol. 1, no. 1, pp. 4-17, 1982.

Schimmels, J.M., and M.A. Peshkin. Force-Assemblability: Insertion of a Workpiece into a Fix/ure Guided by Contact Forces Alone, 1991 IEEE International Conference on Robotics and Automation, 1991.

Sharen, A., N. Hogan, and D.E. Hardt, Controller Design in the Physical Domain (Applicution to Robot Impedance Control)", Proceedings of the 1989 IEEE International Conference on Robotics and Automation, pp. 552-59, 1989.

Sugano, S., S. Tsuto and I. Kato, Force Control of the Robot Finger-Joint Equipped with Mechanical Compliance Adjuster, IEEE/RSJ International Conference on Intelligent Robots and Systems, Raleigh, NC, pp. 2005-13, 1992.

Takesi, O., and M. Honda, Trajectory Formation in Sequential Arm Movements, Proceedings of the 1992 IEEE. International Conference on systems, Man, and Cybernetics, Chicago, IL., pp. 471-478, Oct. 1992.

Tong, J. and J. Somerset, Control, Performance, and Application of Antagonistic Actuated Manipulator Joints, American Control Conference, pp. 63-64, Boston, MA, 1985.

Towhidkhah, F., R.E. Gander, and H.C. Wood, A Stiffness Control Schema for Perturbed and Unperturbed Joint Movement, IEEE WESCANEX 93, Communications, Computers, and Power in the Modern Environment Conference Proceedings, pp. 392-396, 1993.

Uno, Y., M. Kawato, and R. Suzuki, Formation and Control of Optimal Trajectory in Human Multijoint Arm Movement, Biological Cybernetics, vol. 61, pp 89-101, 1989.

Volpe, R., Real and Artificial Forces in the Control of Manipulators: Theory and Experiments, Ph.D. dissertation, Carnegie Mellon Univ., Dept. of Physics, Sept. 1990.

Volpe, R., and P. Khosla, The Equivalence of Second Order Impedance Conirol and Proportional Gain Explicit Force Control: Theory and Experiments, Proceedings of the Second Annual International Symposium on Experimental Robotics, Toulouse, France, June 1991. 
Volpe, R., and P. Khosla, A Theoretical and Experimental Imvestigation of Explicit Force Control Sirategies for Manipulators, IEEE Transactions on Automatic Control, vol. 38, no. 11, pp. 1634-50, Nov. 1993.

Watson, P.C., A Multidimensional System Analysis of the Assembly Process as Performed by a Manipulator, 1st North American Robot Conference, 1976.

Whitney, D.E., Force Feedback Control of Manipulator Fine Motions, ASME Journal of Dynamic Systems, Measurement and Control, pp. 91-97, June 1977.

Whitney, D. E., Historical Perspective and State of the Art in Robot Force Control, The International Journal of Robotics Research, vol. 6, no. 1, pp. 3-13, Spring 1987.

Wiener, N., Cybernetics or Control and Communication in the Animal and Machine, 2nd Edition, Cambridge, MA, M.I.T. Press, 1961.

Wilkes, D.F., Rolamire: A New Mechanical Design Concept, Sandia Corporation Research Report No. SC-RR-67-656, unclassified, Oct. 1967.

Wilkes, D.F., Rolamite: A New Mechantsm, Part I: Nature of the Device: Part 2:

Engineering; Part 3: Applications; Part 4: Hardware, Mechanical Engineering, pp. 1129, April 1968.

Williams III, T.W., The Boston Elbow, SOMA, pp. 29-32, July 1986.

Wilson, A.B., Limb Prosthetics - 1970, Artificial Limbs, no. 14, pp. 1-52, 1970.

Winters, J., L. Stark, and A.H. Seif-Naraghi, An Analysis of the Sources of Musculoskeletal System Impedance, Journal of Biomechanics, vol. 21, no. 12, pp. 1011$1025,1988$.

Wlassich, J.J., Non-Linear Force Feedback Impedance Control, S.M. Thesis, Department of Mechanical Engineering, M.I.T., 1986.

Yi, B. and R.A. Freeman, Synthesis of Actively Adjustable Springs by Antagonistic Redundant Actuation, ASME Journal of Dynamic Systems, Measurement and Control, vol. 114, pp. 454-61, 1992. 


\section{Appendix A: \\ Alternate Proof of Quadratic Springs}

From equation (4-2),

$$
\frac{\partial \psi_{1}}{\partial \delta_{1}}=\frac{\partial \psi_{2}}{\partial \delta_{2}}
$$

Integrating equation (A-1) with respect to $\delta_{1}$,

$$
\psi_{1}+C\left\{\delta_{2}\right\}=\left(\frac{\partial \psi_{2}}{\partial \delta_{2}}\right) \cdot \delta_{1}
$$

Substituting equation (A-1),

$$
\psi_{1}+C\left\{\delta_{2}\right\}=\left(\frac{\partial \psi_{1}}{\partial \delta_{1}}\right) \cdot \delta_{1}
$$

Therefore $C\left\{\delta_{2}\right\}$ is a constant. Renaming $C\left\{\delta_{2}\right\}$ as $C_{1}$ and separating the variables in equation (A-3),

$$
\frac{d \psi_{1}}{\psi_{1}+C_{1}}=\frac{d \delta_{1}}{\delta_{1}}
$$

Integrating equation (A-4),

$$
\ln \left(\psi_{1}+C_{1}\right)=\ln \left(\delta_{1}\right)+C_{2}
$$

Transforming equation (A-S) to exponential form and simplifying,

$$
\psi_{1}+C_{1}=\delta_{1} \cdot e^{c_{2}}=C_{3} \delta_{1}
$$

Rearranging,

$$
\Psi_{1}=C_{3} \delta_{1}-C_{1}
$$

Similarly,

$$
\Psi_{2}=C_{1} \delta_{2}+C_{3}
$$

Thus the stiffnesses must vary linearly as in equations (4-17a) and (4-17b). This means the force functions must be quadratic. Note that this method is simpler, but it does not follow the graphical proof in Section 4.1.1. 


\section{Appendix B: \\ State Space Representation of Configurations}

\section{Configuration 1}

The bond graph for the configuration is shown in Figure B.1. It is identical to Figure 3.3 with symmetry and the links numbered.

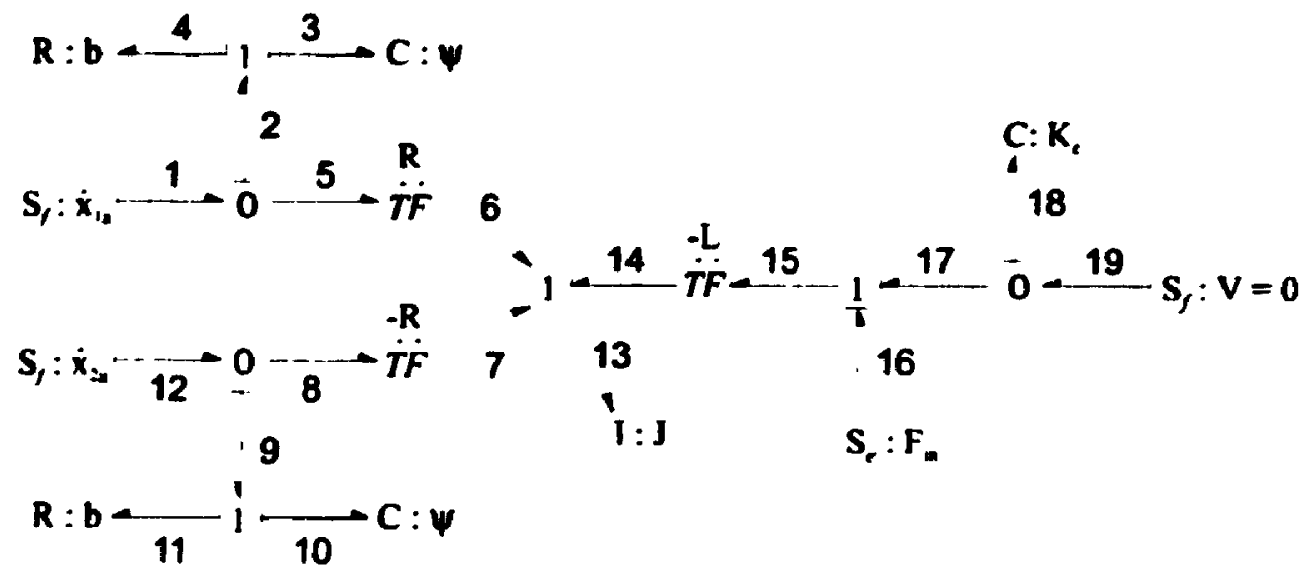

Figure B. 1: Bond graph of Configuration 1

States: $\mathbf{x}_{3}, \mathbf{x}_{10}, \mathbf{x}_{18}, \mathbf{P}_{\mathfrak{1 3}}$

Inputs: $\dot{x}_{1 a}, \dot{x}_{2 a}, F_{n n}, v$

Constitutive equations:

$$
\begin{aligned}
& F_{3}=\phi\left\{x_{3}\right\} \\
& F_{10}=\phi\left\{x_{10}\right\} \\
& \omega_{13}=\frac{p_{113}}{J} \\
& F_{18}=K_{e} x_{18} \\
& \dot{x}_{3}=v_{2}=\dot{x}_{14}-v_{3}=\dot{x}_{12}-R \omega_{6}=\dot{x}_{12}-R \omega_{13}=\dot{x}_{10}-\frac{R}{J} p_{x 13} \\
& \dot{x}_{10}=v_{9}=\dot{x}_{21}-v_{3}=\dot{x}_{21}+R \omega_{7}=\dot{x}_{21}+R \omega_{13}=\dot{x}_{22}+\frac{R}{J} p_{113} \\
& \dot{x}_{12}=v_{19}-v_{17}=V-v_{15}=V+L \omega_{14}=V+L \omega_{13}=V+\frac{L}{J} p_{113}
\end{aligned}
$$




$$
\begin{aligned}
& \dot{p}_{13}=\tau_{6}+\tau_{7}+\tau_{14}=R F_{3}-R F_{2}-L F_{15}=R F_{2}-R F_{9}-L\left(F_{16}+F_{17}\right) \\
& =R\left(\phi\left\{x_{3}\right\}+b v_{4}\right)-R\left(\phi\left\{x_{10}\right\}+b v_{11}\right)-L\left(F_{w 2}+F_{18}\right) \\
& v_{4}=v_{2}=\dot{x}_{1 e}-\frac{R}{J} p_{n 13} \\
& v_{11}=v_{9}=\dot{x}_{2 a}+\frac{R}{J} p_{113} \\
& \dot{p}_{z 13}=R\left(\phi\left\{x_{3}\right\}-\phi\left\{x_{10}\right\}\right)+b\left(\dot{x}_{1 a}-\dot{x}_{2 a}\right)-\frac{2 R b}{J} p_{z 13}-L\left(F_{t 10}+K_{c} x_{18}\right)
\end{aligned}
$$

In matrix form, the state space representation of the configuration is

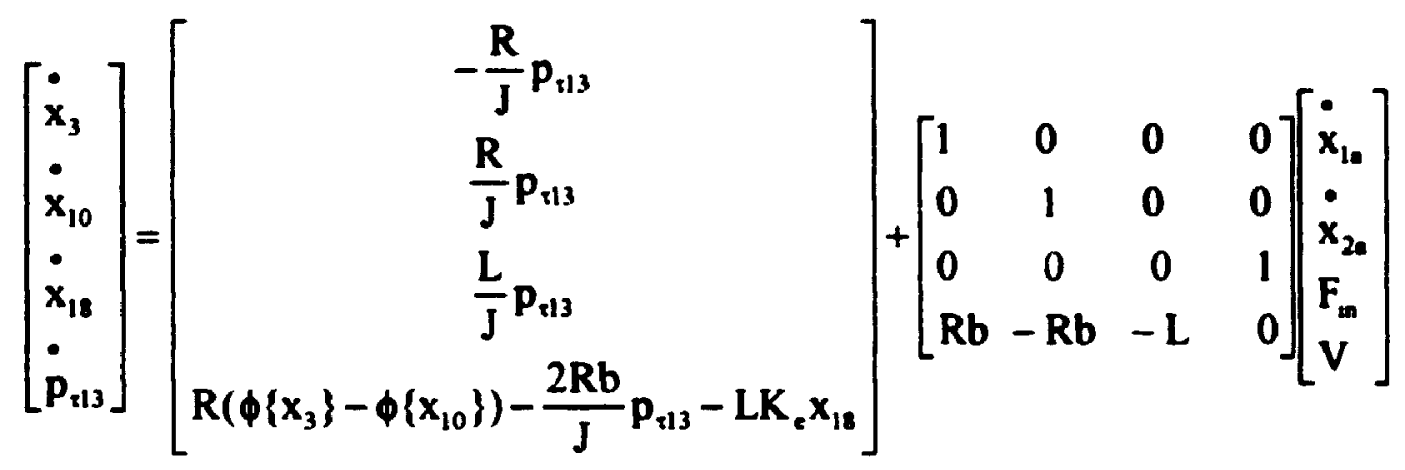

Substituting the parameters used in the design,

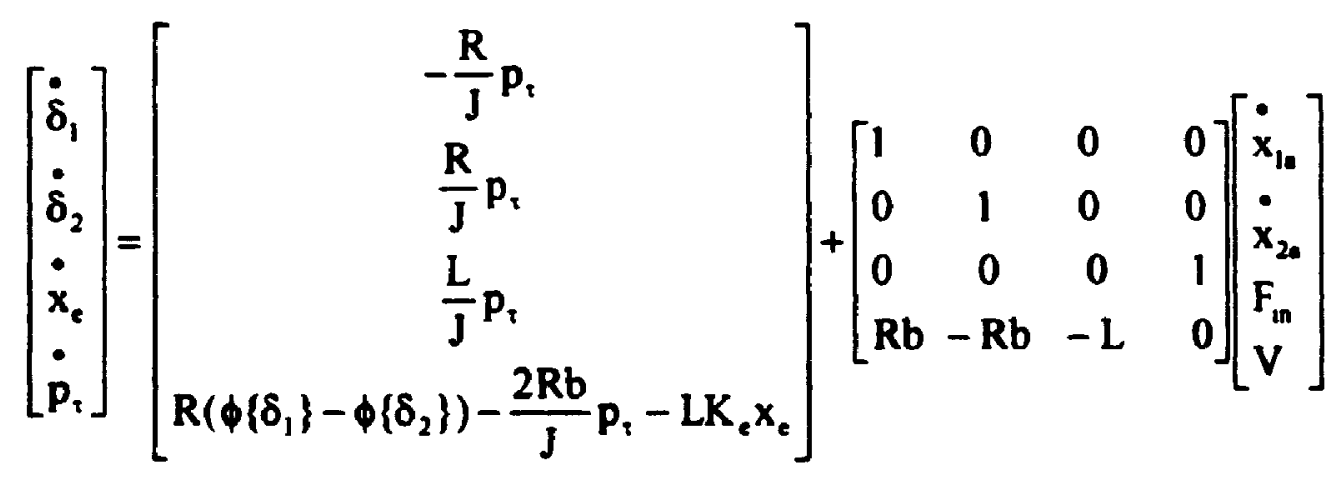

where $p_{z}$ is the angular momentum of the joint, $x_{e}$ is the displacement of the environment, $F_{\text {in }}$ is loading due to weight, and $V$ is the velocity of the environment. 


\section{Configuration 2}

The bond graph for the configuration is shown in Figure B.2. It is identical to Figure 3.11(a) with symmetry.

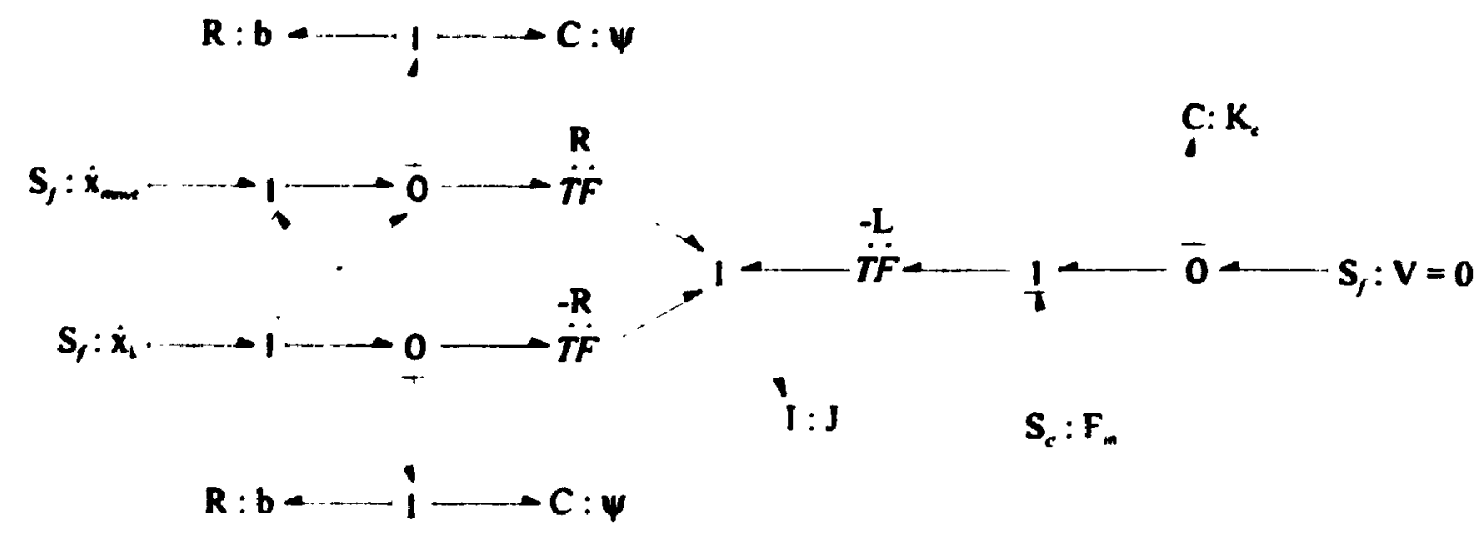

Figure B.2: Bond graph of Configuration 2.

The state space representation is similar to that of Configuration 1 with a change of inputs.

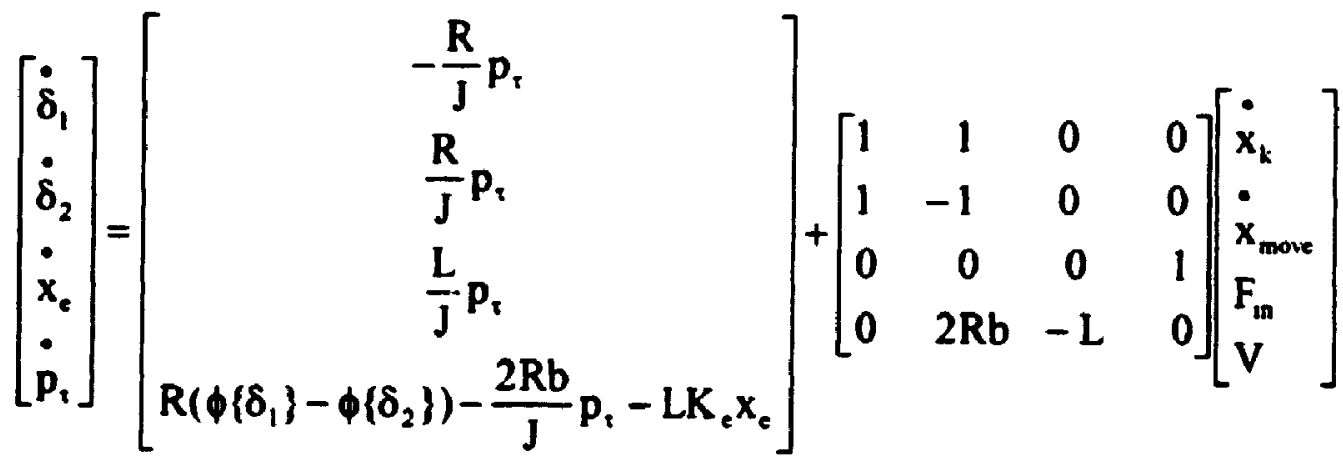




\section{Configuration 3}

The bond graph for the configuration is shown in Figure B.3. It is similar to Firure 3.5(a) with symmetry and a change of inputs.

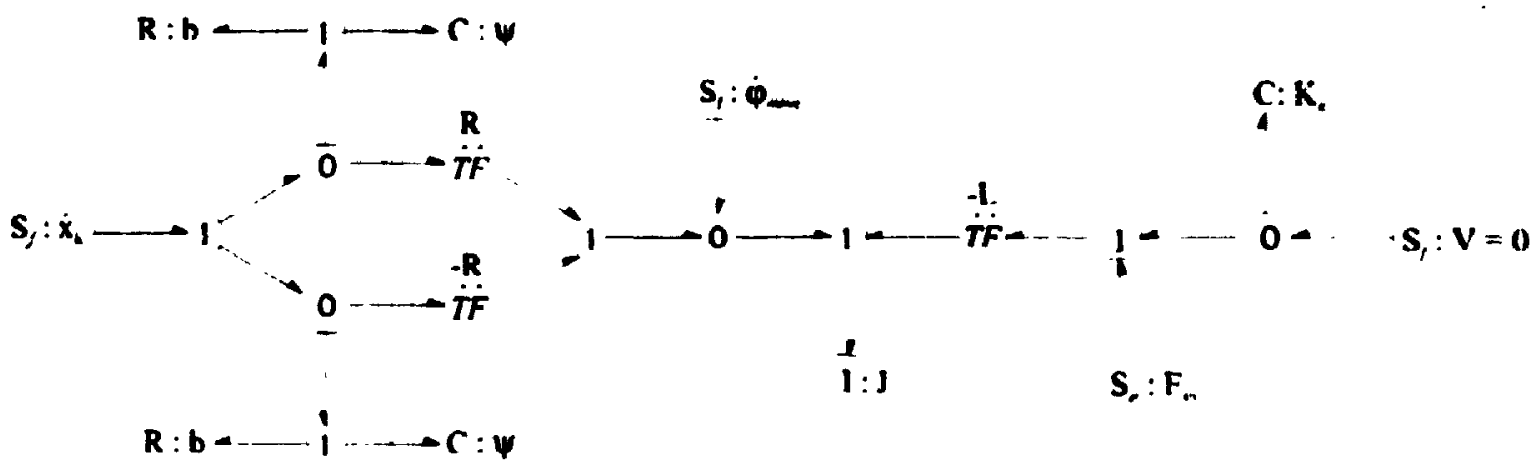

Figure B.3: Bond graph of Configuration 3

Again, the state space representation is similar to that of Configurations 1 and 2 with a change of inputs.

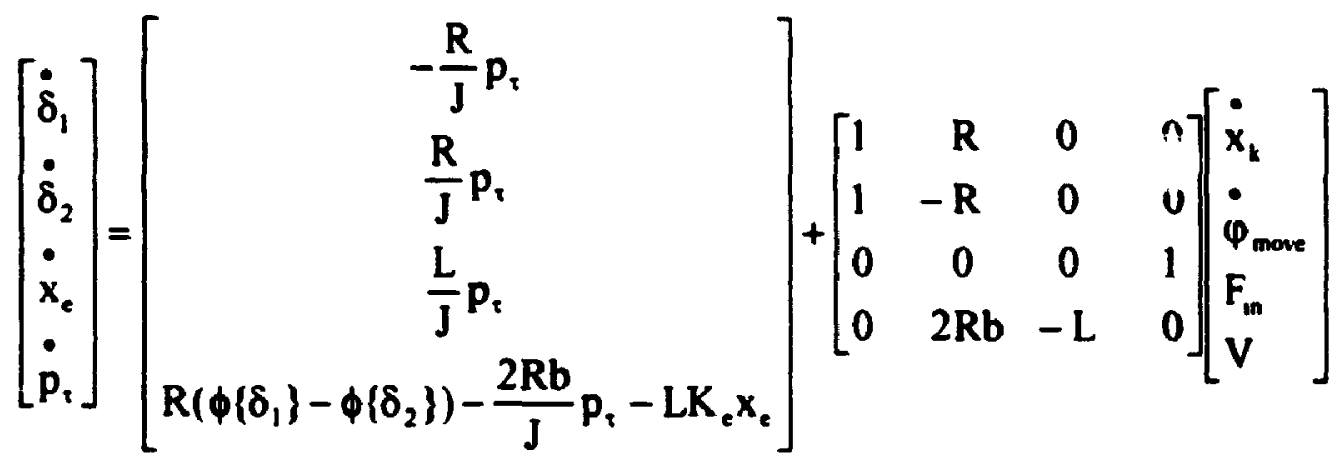

\section{Configuration 4}

This configuration is identical to Configuration 3 . The only difference is the selection of parameters. 


\section{Appendix C: \\ MATLAB Simulation Code}

\section{Common Files:}

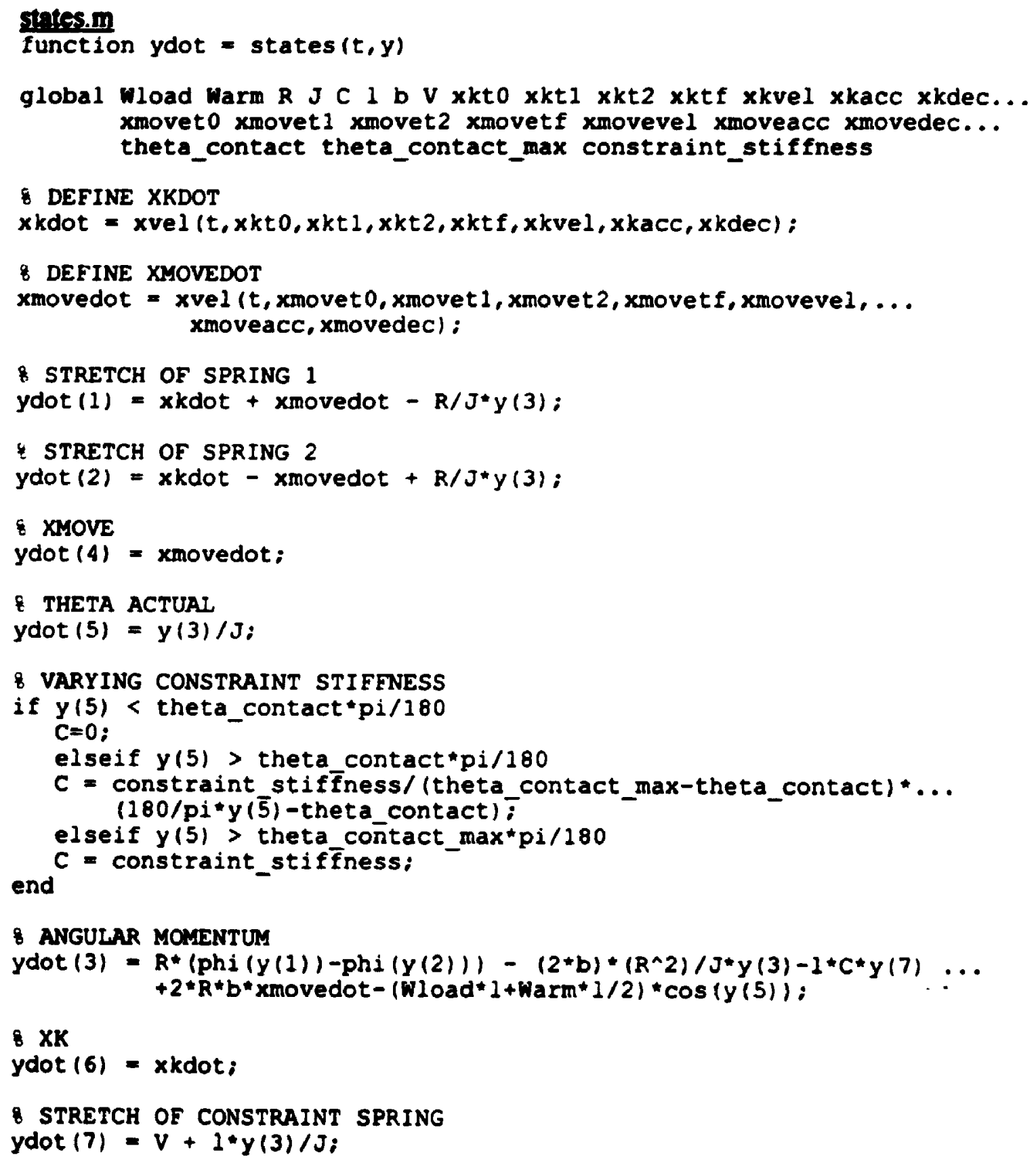


phim

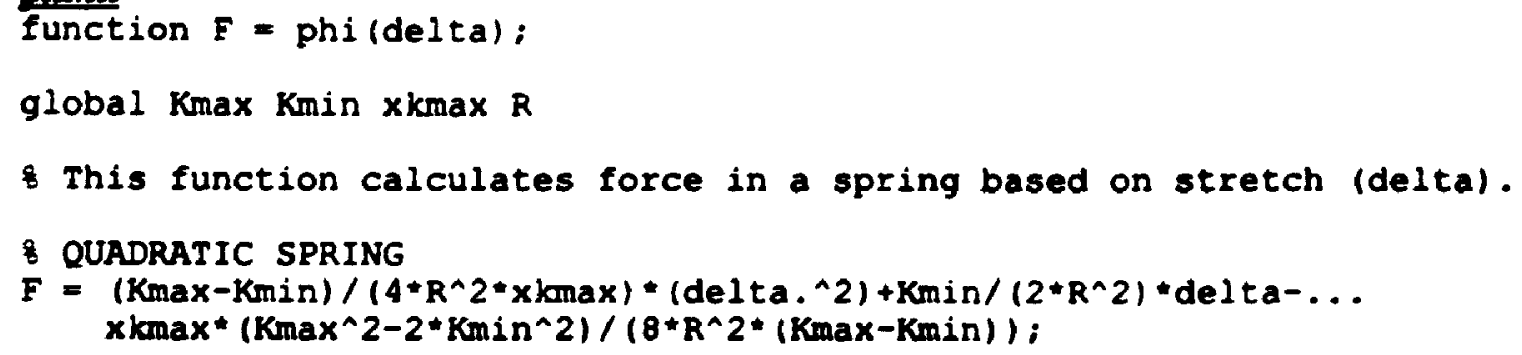

psi.m

function $k=$ psi (delta)

global Kmax Kmin R xkmax Kset

This function calculates the stiffness of a spring based on stretch (delta).

5 QUADRATIC SPRING

$k=(K \max -K \min ) /\left(2 * R^{\wedge} 2 \star x k \max \right) * \operatorname{del} t a+K \min /\left(2 * R^{\wedge} 2\right)$;

\section{xvel.m}

function xvelocity $=x v e l(t, t 0, t 1, t 2, t f, v \max$, acc, dec $)$

This function calculates a desired velocity matrix based on knowing

when motion starts $(t 0)$, when it stops $(t f)$, the acceleration (acc),

the deceleration (dec), a constant velocity (umax) between the acc.

and dec.. the time (t1) at which vmax is reach by accerating, and the

time (t2) to begin decfieration in order to stop at $t f$.

xvelocity $=\operatorname{acc}^{\star}(t-t 0) .(t>=t 0 \& t<=t 1)+\operatorname{vmax} *(t>t 1 \& t<=t 2 ; \ldots$ $+\left(\operatorname{dec}^{*}(t 2-t)+v \max \right) *(t>t 2 \& t<=t f)+0 *(t<t 0)+0 *(t>t f)$;

\section{define.m}

global Kmax Kmin xkmax $R J$ wload Warm 1 b $V$ to tf xki xkto xkt $1 .$. xkt2 xktf xkvel xkacc xmoveto xmovet1 xmovet 2 xmovetf... xmovevel xmovei xmoveacc xmovedec xkf xkdec theta_contact... theta_contact_max constraint_stiffness $x_{-}$contact

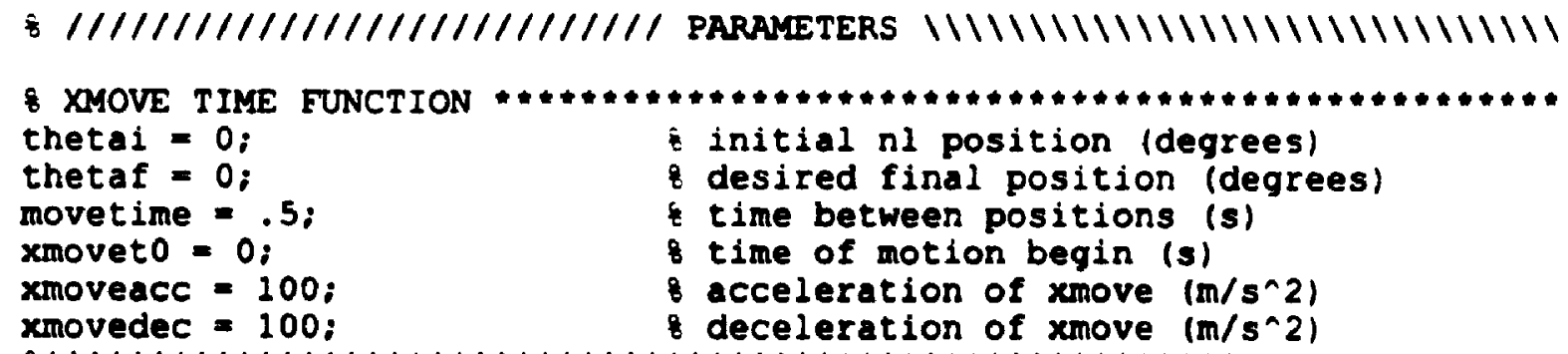

initial $K$ setting (N.m/rad)

E Inal $\mathrm{K} *$ setting (N.m/rad)

time of stifiness change (s) 


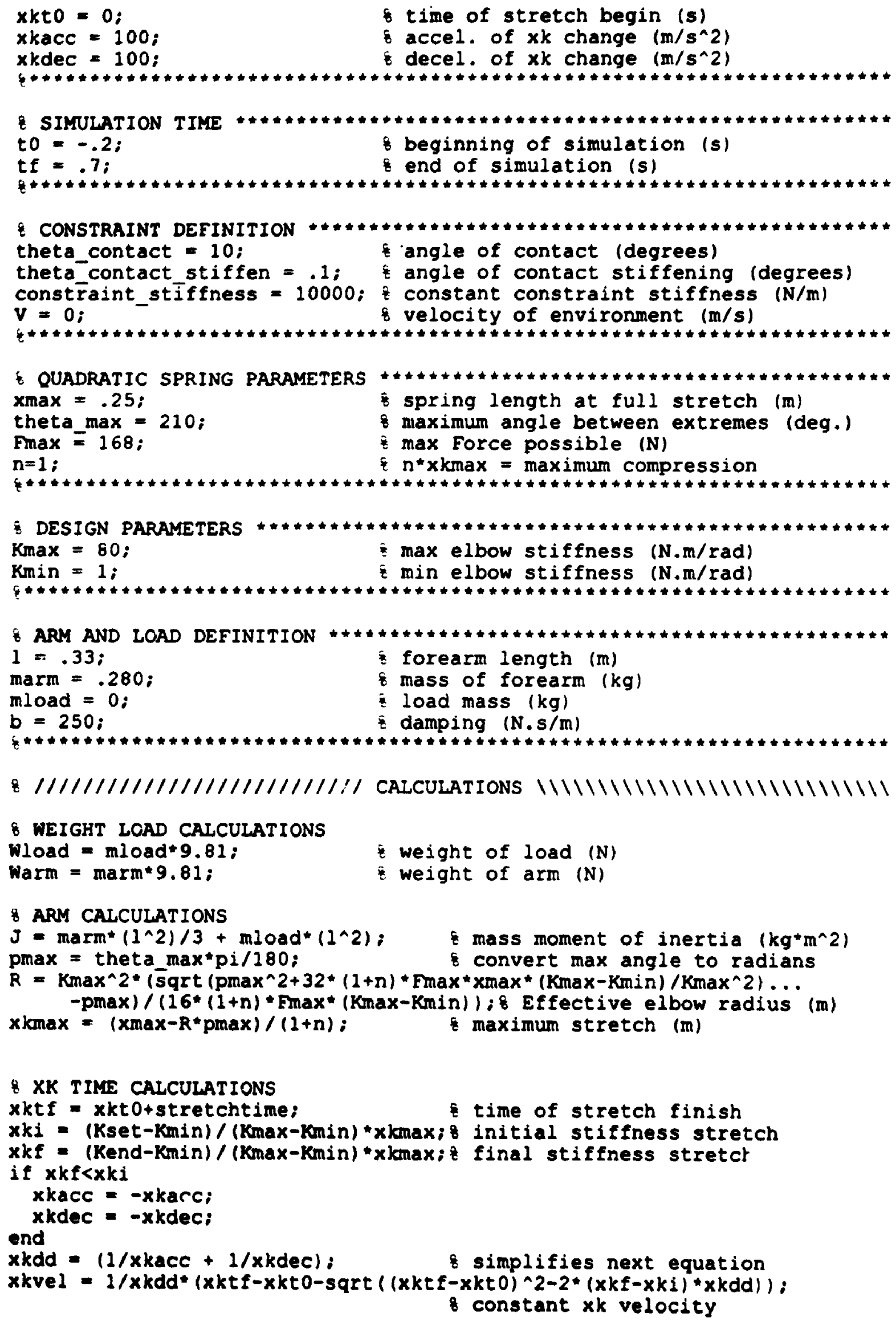




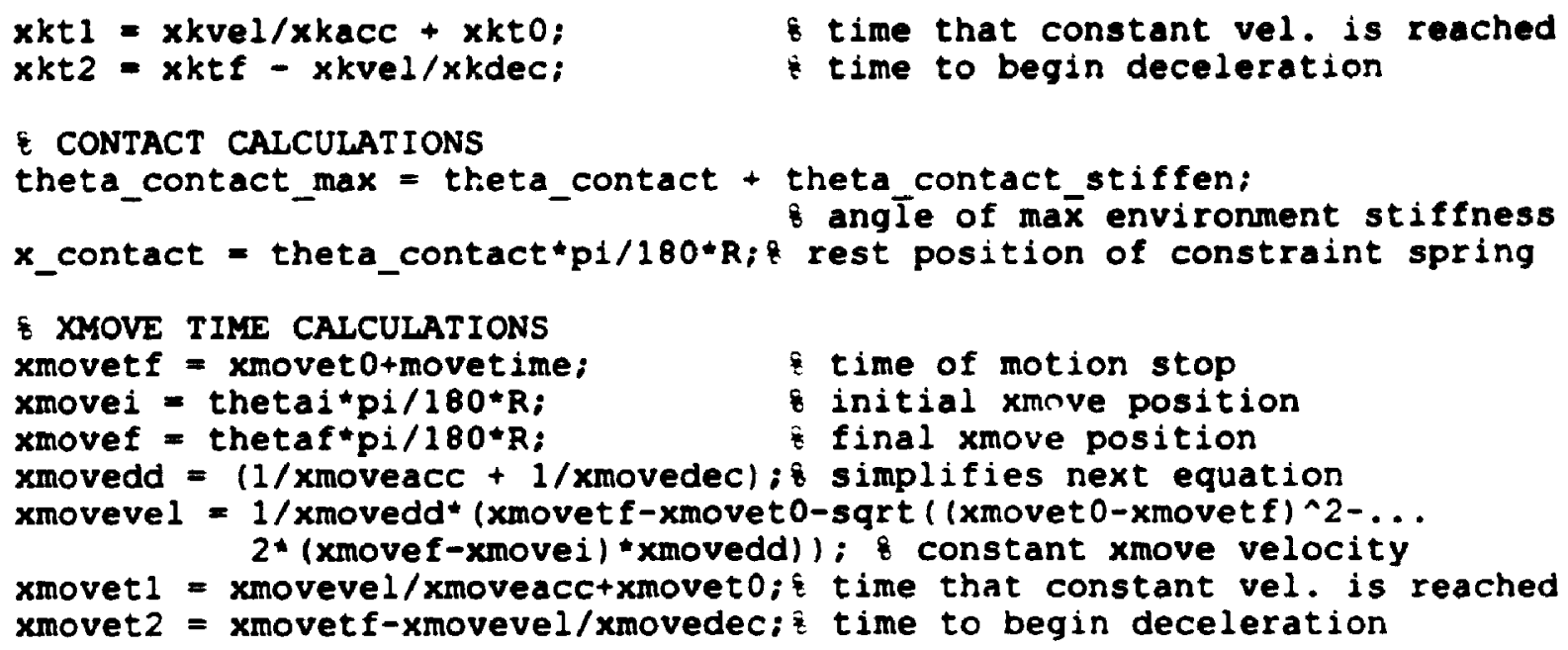

\section{Configuration 1: \\ configl.m}

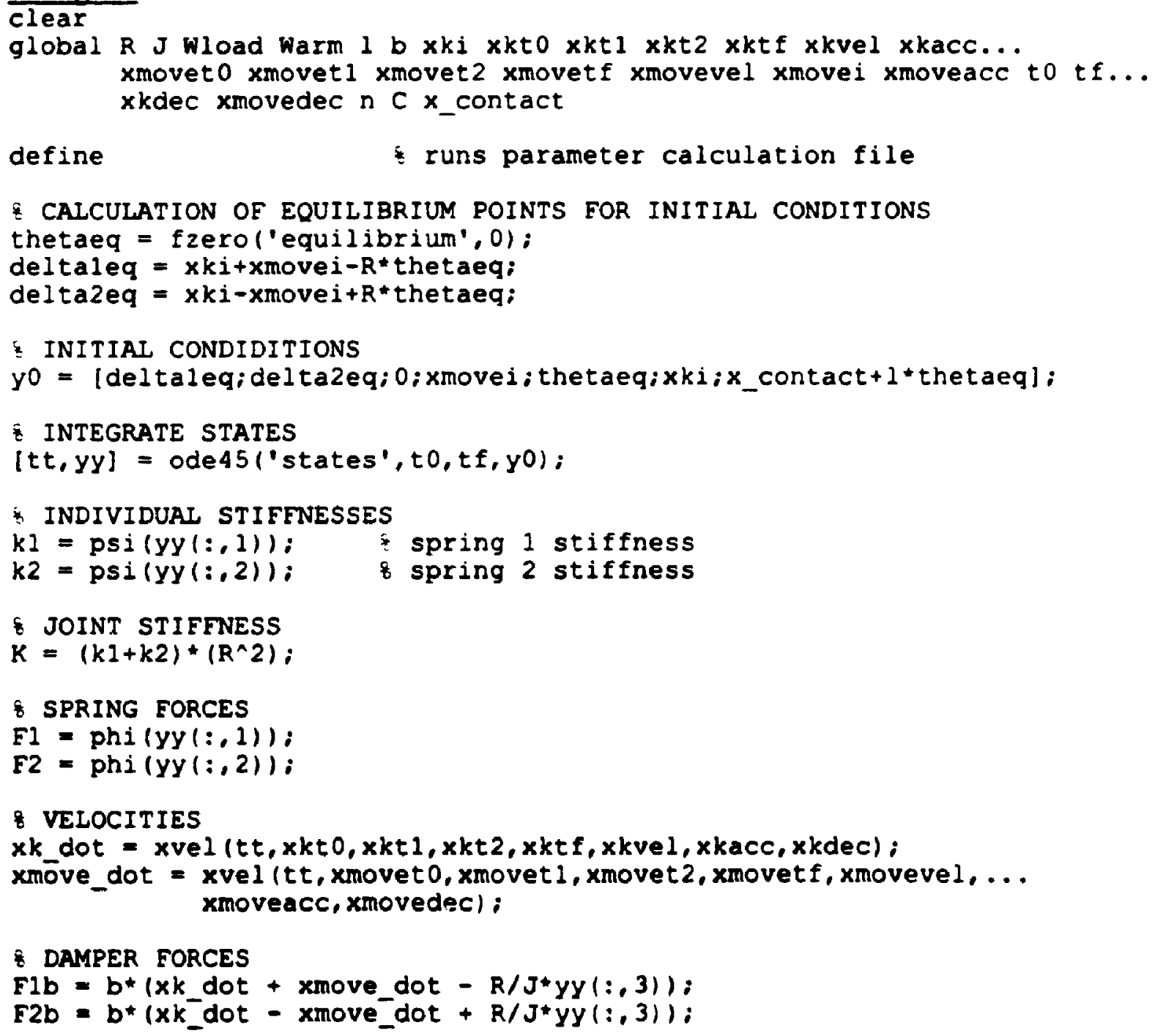




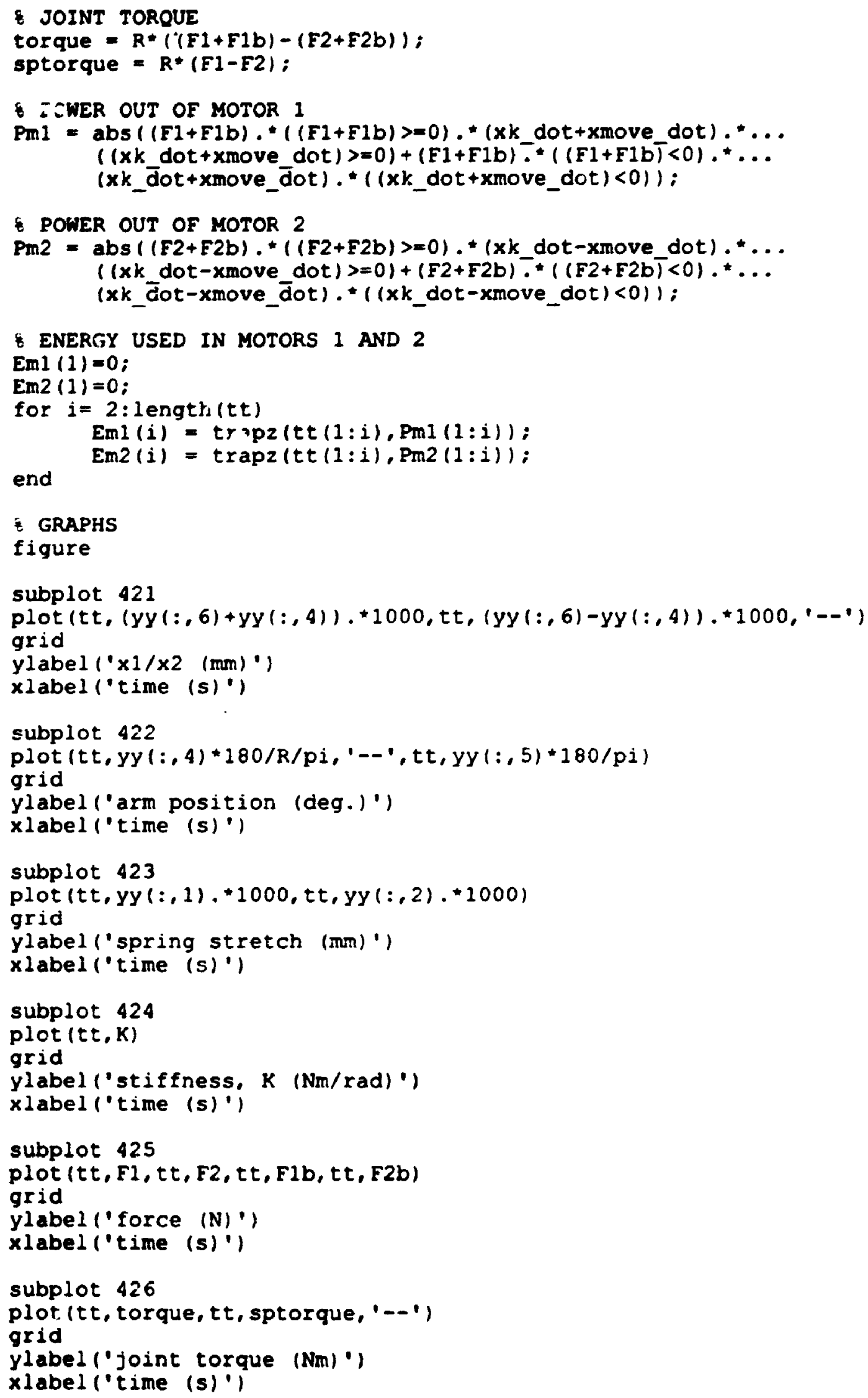




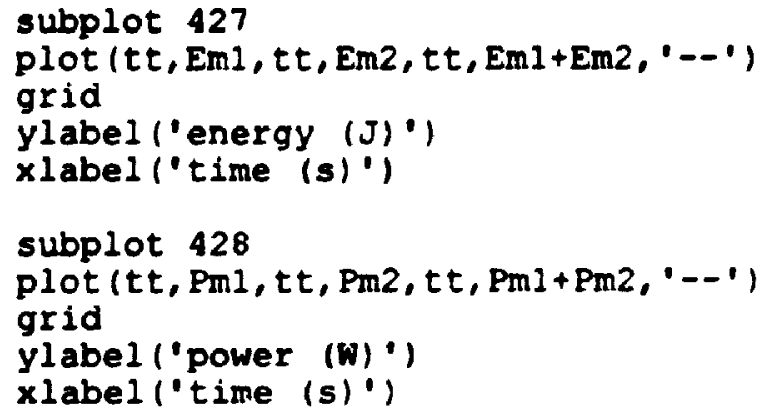

\section{Configuration 2:}

config2.m

\section{clear}

global $R \mathrm{~J}$ Wload Warm 1 b xki xkt0 xkt1 xkt2 xktf xkvel xkacc... xmoveto xmovet 1 xmovet 2 xmovetf xmovevel xmovei xmoveacc to tf... xkdec xmovedec n C x_contact

define runs parameter calculation file

₹ CALCULATION OF EQUILIBRIUM POINTS FOR INITIAL CONDITIONS

thetaeq = fzero('equilibrium',0);

deltaleq $=x k i+x m o v e i-R *$ thetaeq;

delta2eq $=x k i-x m o v e i+R *$ thetaeq;

INITIAL CONDIDITIONS

yo = [deltaleq;delta2eq; $0 ; x$ movei; thetaeq; xki; $x$ contact +1 *thetaeq] :

* INTEGRATE STATES

$[t t, y y]=$ ode45('states',to,tf,y0,1.e-6,0);

\& INDIVIDUAL STIFENESSES

$k 1=$ psi(yy(:,1)); spring 1 stiffness

$k 2=$ psi $(y y(:, 2)):$ spring 2 stiffness

JOINT STIFENESS

$k=(k 1+k 2) *\left(R^{\wedge} 2\right)$;

SPRING FORCES

$F 1=\operatorname{phi}(Y y(:, 1)):$

$F 2=\operatorname{phi}(y y(:, 2)) ;$

$\varepsilon$ VELOCITIES

$x k$ dot $=x v e l(t t, x k t 0, x k t 1, x k t 2, x k t f, x k v e l, x k a c c, x k d e c)$;

xmove_dot $=$ xvel $(t t$, xmovet $0, x$ movet 1, xmovet 2, xmovet $f, x m o v e v e 1, \ldots$ xmoveacc, xmovedec):

\& DAMPER FORCES

$F 1 b=b_{0}^{*}(x k$ dot $+x m o v e$ dot $-R / J * y y(:, 3))$;

$F 2 b=b^{*}\left(x k\right.$ _dot $\left.-x m o v e \_d o t+R / J * y y(:, 3)\right)$;

JOINT TORQUE

torque $=R *((F 1+F(b)-(F 2+F 2 b)):$

sptorque $=R^{*}(F 1-F 2)$ :

POWER OUT OF $K$ (STIFENESS) MOTOR

$P k=a b s((F)+F 1 b+F 2+F 2 b) . *((F 1+F 1 b+F 2+F 2 b)>=0) . * x k_{-} d o t . *(x k$ dot $>=0) \ldots$ 
$+(F 1+F 1 b+F 2+F 2 b) \cdot *((F 1+F 1 b+F 2+F 2 b)<0) .{ }^{*} x k$ dot $\left.\cdot{ }^{*}\left(x k \_\operatorname{dot}<0\right)\right) ;$

8 PONER OUT OF MOVE MOTOR

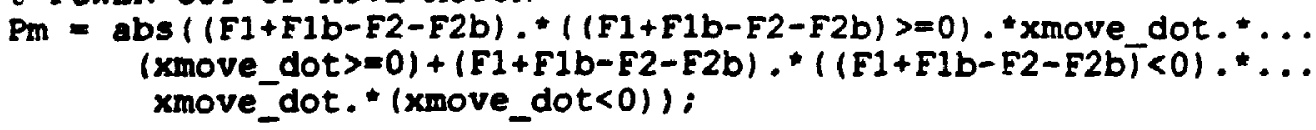

\& ENERGY USED IN STIFENESS AND MOVE MOTORS

Ek(1) $=0$;

$\operatorname{Em}(1)=0$;

for $i=2: \max (s i z e(t))$

$E k(i)=\operatorname{trapz}(t t(1: i), \operatorname{Pk}(1: i))$ :

end

$\operatorname{Em}(i)=\operatorname{trapz}(\operatorname{tt}(1: i), \operatorname{Pm}(1: i)) ;$

G GRAPHS

figure

subplot 421

plot $(t t, y y(:, 6), \star 1000, \cdots-t$, tt, yy $(:, 4), \star 1000)$

grid

ylabel ('xk/xmove (mm)')

xlabel ('time (s)')

subplot 422

plot $\left(t t, y y(:, 4) * 180 / R / p i,{ }^{\prime}-{ }^{\prime}, t t, y y(:, 5) * 180 / p i\right)$

grid

ylabel ('arm position (deg.)')

xlabel ('time (s)')

subplot 423

plot $(t t, y y(:, 1)+* 1000, t t, y y(:, 2), * 1000)$

grid

ylabel("spring stretch (mm)")

xlabel ('time (s)')

subplot 424

$\operatorname{plot}(t t, K)$

grid

ylabel ('stiffness, $K(\mathrm{Nm} / \mathrm{rad})$ ')

xlabel ('time (s)')

subplot 425

Plot (tt, F1, tt, F2, tt, F1b, tt, F2b)

grid

ylabel ('force (N)')

xlabel ('time (s)')

subplot 426

plot (tt, torque, $t t$, sptorque, '--')

grid

ylabel ("Joint torque (Nm)")

$x$ label ("time (s)')

subplot 427

plot (tt, Ek, tt, Em, tt, Ek+Em, ---1 )

grid

ylabel ('energy (J)')

xlabel ('time (s)')

subplot 428 


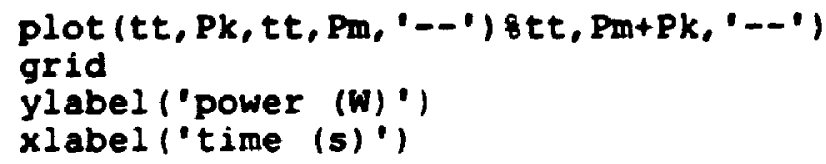

\section{Configuration 3:}

\section{confie3.m}

clear

global $\mathrm{R} J$ ilload ham 1 b xki xkt0 xkt1 xkt2 xktf xkvel xkacc... xmoveto xmovet 1 xmovet 2 xmovetf xmovevel xmovei xmoveacc to tf... xkdec xmovedec n C $x_{\text {_contact }}$

define runs parameter calculation file

* CALCULATION OF EQUILIBRIUM POINTS FOR INITIAL CONDITIONS thetaeq = fzero("equilibrium',0):

deltaleq = xki+xmovei-R*thetaeq;

delta2eq = xki-xmovei+R* thetaeq;

INITIAL CONDIDITIONS

y0 = [deltaleq;delta2eq:0;xmovei; thetaeq;xki;x_contact+1 *thetaeq];

\& INTEGRATE STATES

$[t t, y y]=$ ode $45\left(^{\prime}\right.$ states',to,tf, y0,1,e-6,0);

\& INDIVIDUAL STIEFNESSES

$k 1=p s i(y y(:, 1)) ; \quad$ spring 1 stiffness

$k_{2}=\operatorname{psi}(y Y(:, 2)):$ spring 2 stiffness

SOINT STIFFNESS

$K=(k 1+k 2) *\left(R^{\wedge} 2\right)$;

\& VIOCITIES

$x k$ dot $=x v e l(t t, x k t 0, x k t 1, x k t 2, x k t f, x k v e l, x k a c c, x k d e c)$;

xmove_dot $=x v e l(t t, x m o v e t 0, x m o v e t 1, x m o v e t 2, x m o v e t f, x m o v e v e 1, \ldots$ xmoveacc, xmovedec):

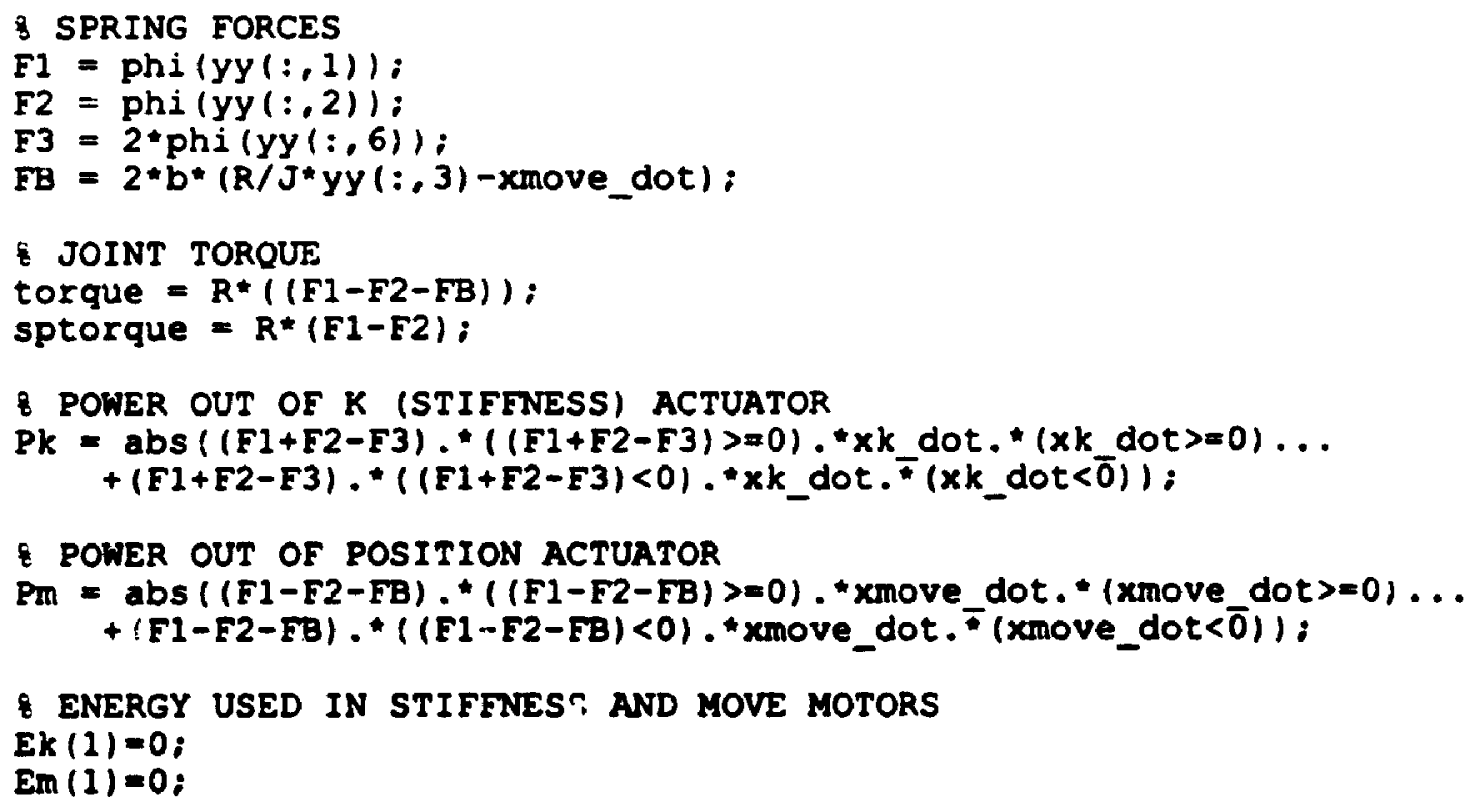




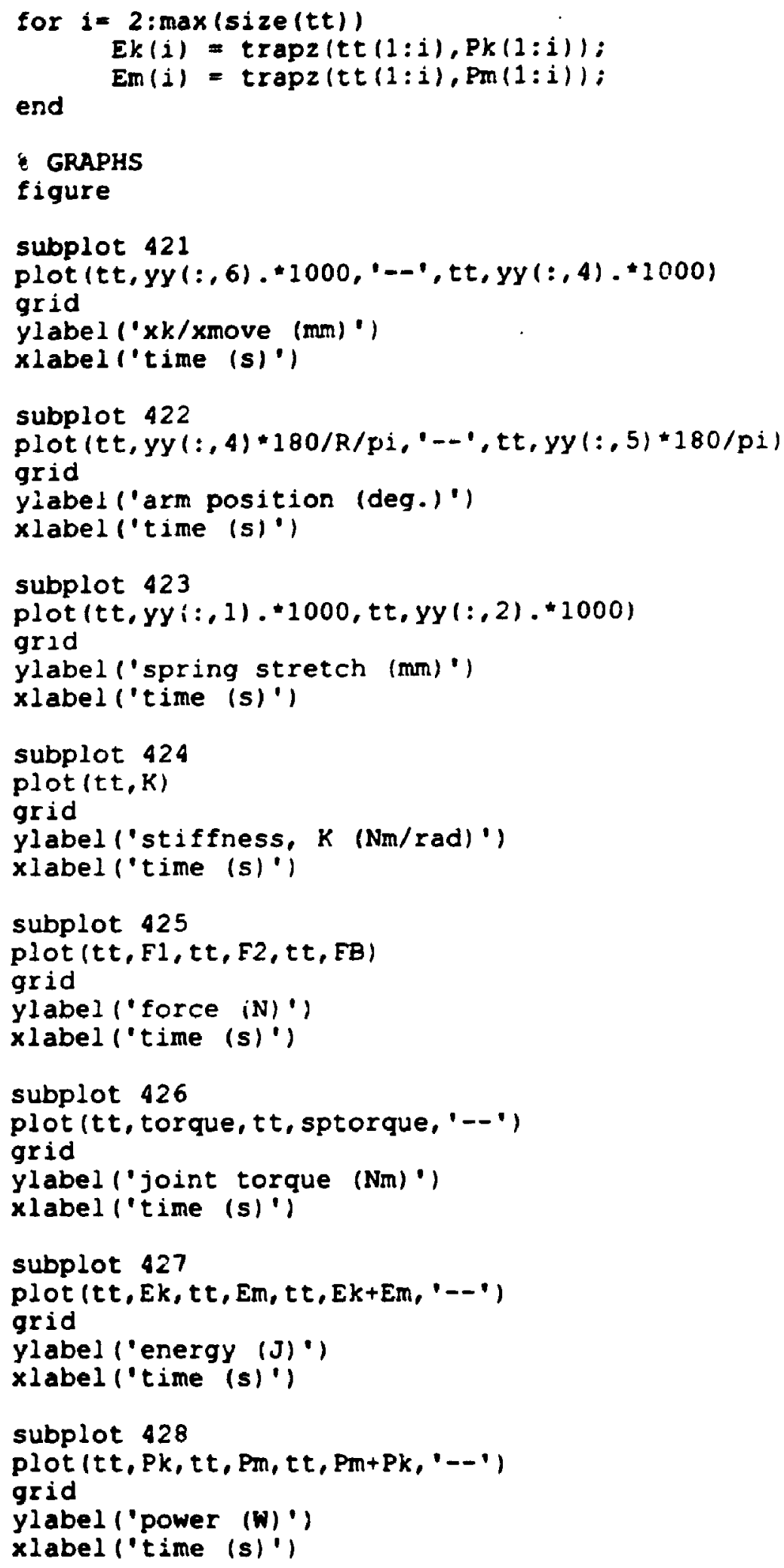

\section{Configuration 4:}

uses config3.m, changes are made in define. $m$. 
Task 1:

Configuration 1: - as above

Configuration 2: - as above

Configuration 3:

changes to define.m

theta_max $=0$;

Fmax $\bar{x} 50.56$;

$b=20$

Configuration 4: - as above

\section{Task 2:}

Configuration 1:

changes to configl.m

yo $=[$ xki; xki; 0 ; xmove $i$; xmovei $/ R ; x k i ; x$ contact 1 *xmove $1 / R]$;

changes to states.m

$y \operatorname{dot}(3)=R^{*}(\operatorname{phi}(y(1))-\operatorname{ph}(y(2)))-(2 * b) *\left(R^{\wedge} 2\right) / J * y(3)-1 * c^{*} y(7) \ldots$ $+2 * R * b * x$ movedot

Configuration 2: - changes as in Configuration 1

Configuration 3: - changes as in Configuration 1 and Task 1

Configuration 4: - changes as in Configuration 1

Task 3:

Configuration 1: - chariges as in Task 2

changes to define.m

thetai $=-5 ;$

thetaf $=5$

Kset $=50$;

Configuration 2: - changes as in Configuration 1

Configuration 3: - changes as in Configuration 1 and Task 1

Configuration 4: - changes as in Configuration 1

Task 4:

Configuration 1: - changes as in Task 2

changes to define.m

Kset $=50$;

Kend $=1 ;$

xkt0 $=0.25$;

tf $=.9$;

theta_contact $=0$;

Configuration 2: - changes as in Configuration 1

Configuration 3: - changes as in Configuration 1 and Task 1

Configuration 4: - changes as in Configuration 1 

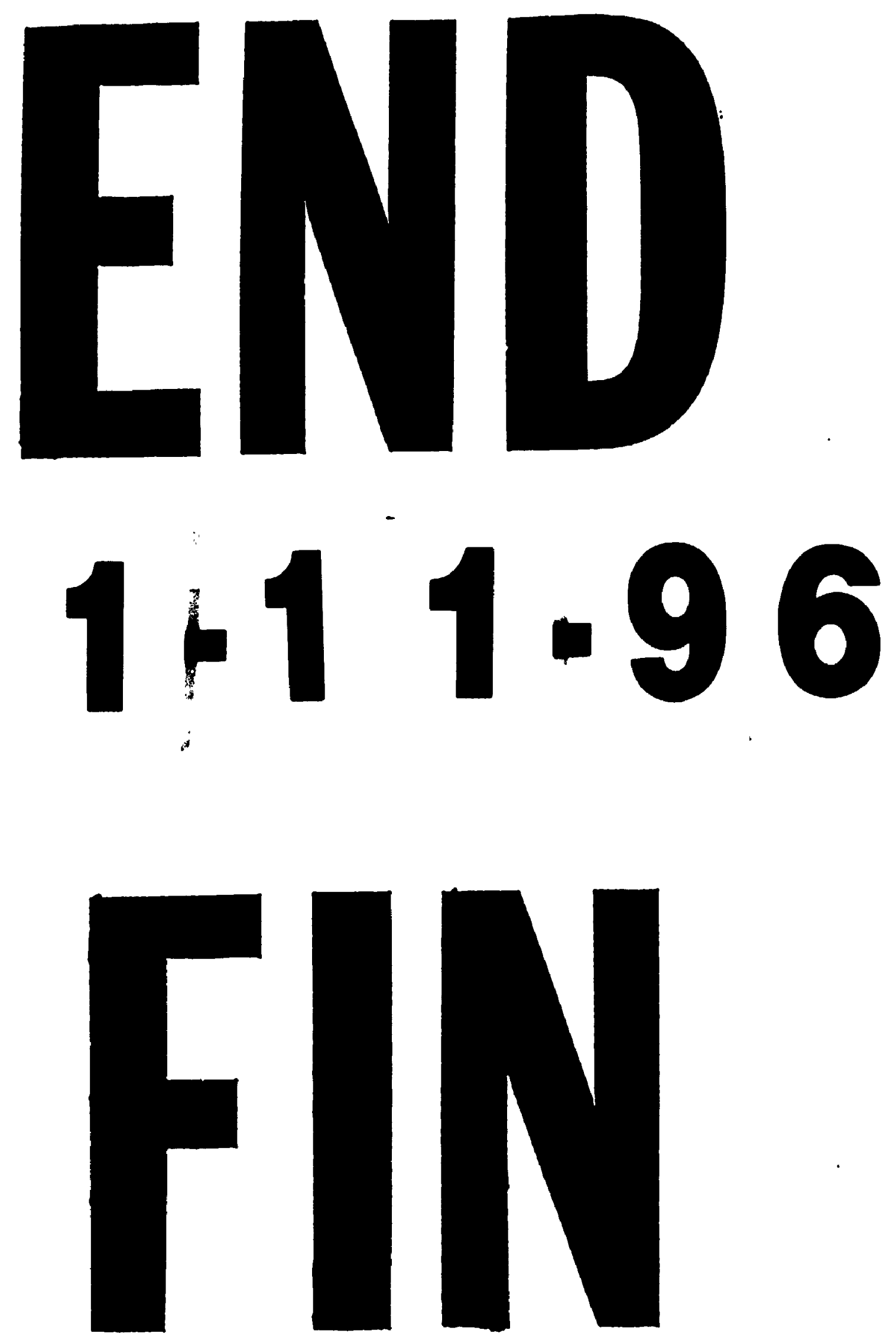